Supporting Information

\title{
Enantio- and Regioselective CuH-Catalyzed Conjugate Reduction of Yne-Allenones
}

\author{
Jia-Yin Wang ${ }^{1}$, Guigen $\mathrm{Li}^{1,2 *}$, Wen-Juan $\mathrm{Hao}^{3}$ \& Bo Jiang ${ }^{3 *}$ \\ ${ }^{1}$ Institute of Chemistry \& BioMedical Sciences, Collaborative Innovation Center of Chemistry for Life Sciences, \\ Nanjing University, Nanjing 210093, P. R. China \\ ${ }^{2}$ Department of Chemistry and Biochemistry, Texas Tech University, Lubbock, Texas 79409-1061, United States; \\ email: guigen.li@ttu.edu (G.L.). \\ ${ }^{3}$ School of Chemistry \& Materials Science, Jiangsu Key Laboratory of Green Synthetic Chemistry for Functional \\ Materials, Jiangsu Normal University, Xuzhou 221116, P. R. China; email jiangchem@jsnu.edu.cn (B.J.)
}

\section{Context}

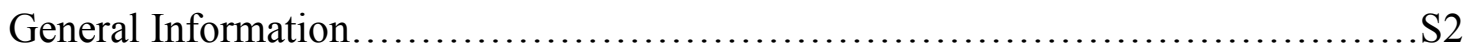

X-Ray Absolute Configuration Structure of Product 3c.........................S2

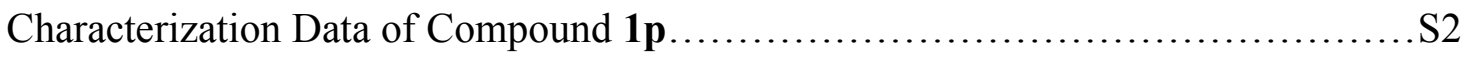

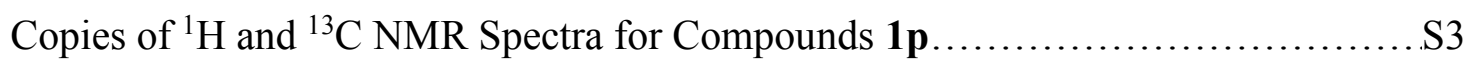

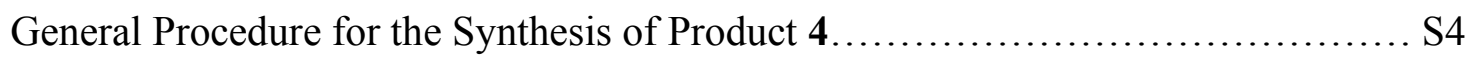

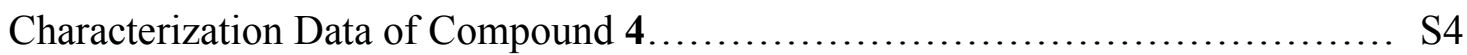

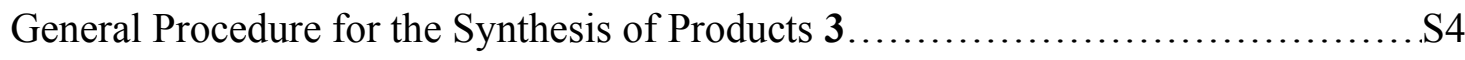

Characterization Data of Compounds 3a-3dd................................S4-S14

Copies of Chiral HPLC Analyses of Products 3a-3dd and 4 .......................S15-S44

Copies of ${ }^{1} \mathrm{H}$ and ${ }^{13} \mathrm{C}$ NMR Spectra for Compounds 3a-3dd and 4 ..................S45-S104 


\section{Experimental}

\section{General Information}

${ }^{1} \mathrm{H}$ NMR $\left({ }^{13} \mathrm{C}\right.$ NMR) spectra were measured on a Bruker DPX $400 \mathrm{MHz}$ spectrometer in $\mathrm{CDCl}_{3}$ (DMSO- $d_{6}$ ) with chemical shift $(\delta)$ given in ppm relative to TMS as internal standard [ $(\mathrm{s}=$ singlet, $\mathrm{d}=$ doublet, $\mathrm{t}=$ triplet, brs $=$ broad singlet, $\mathrm{m}=$ multiplet), coupling constant $(\mathrm{Hz})]$. HRMS (ESI) was determined by using microTOF-QII HRMS/MS instrument (BRUKER). X-Ray crystallographic analysis was performed with a Siemens SMART CCD and a Siemens $\mathrm{P} 4$ diffractometer.

Yne-Allenones were prepared according to literature procedures (See J. Org. Chem. 2009, 74, 2224 and Angew. Chem., Int. Ed. 2017, 56, 15570). Dimethoxy(methyl)silane, chiral ligands $\left(\mathbf{L}_{\mathbf{1}}-\mathbf{L}_{\mathbf{8}}\right)$ and other reagents, unless otherwise noted, were purchased from commercial vendors and used without further purification. The single-crystal of $(R)-\mathbf{3 c}$ was grown from the mixed solution of ethanol and acetone $(\mathrm{V} / \mathrm{V}=3: 1)$.

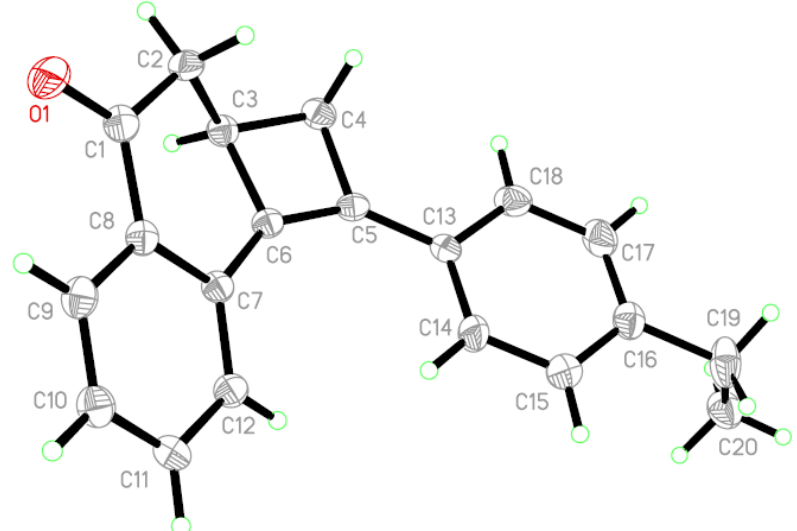

Figure S1. The ORTEP Drawing of Absolute Configuration of Product $(R)-3 \mathbf{c}$

\section{1-(2-(3,3-dimethylbut-1-yn-1-yl)phenyl)buta-2,3-dien-1-one (1p)}<smiles>C=CC(=O)c1ccccc1C#CC(C)(C)C</smiles>

${ }^{1} \mathrm{H}$ NMR (400 MHz, DMSO) $(\delta, \mathrm{ppm}): 8.00(\mathrm{~d}, J=16.0 \mathrm{~Hz}, 1 \mathrm{H}), 7.85-7.81(\mathrm{~m}, 1 \mathrm{H}), 7.43-7.41(\mathrm{~m}, 1 \mathrm{H}), 7.31(\mathrm{~d}, J=16.0$ $\mathrm{Hz}, 1 \mathrm{H}), 6.20-6.17$ (m, 1H), 5.64 (d, $J=6.4 \mathrm{~Hz}, 2 \mathrm{H}), 1.34$ (s, 9H). ${ }^{13} \mathrm{C}$ NMR (100 MHz, DMSO) ( $\left.\delta, \mathrm{ppm}\right): 216.3,188.3$, 139.9, 135.5, 132.8, 130.8, 128.9, 126.8, 124.7, 124.4, 105.5, 96.5, 81.1, 77.2, 31.0, 28.4. 


\section{General procedure for the synthesis of compound 4}

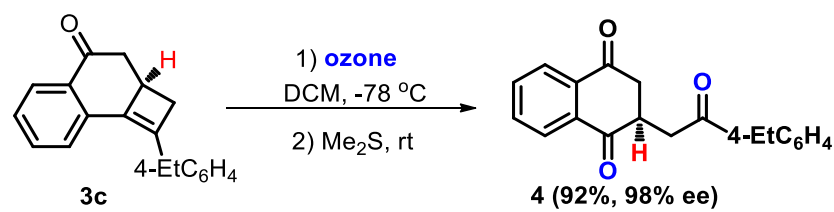

A solution of $3 \mathbf{c}(0.1 \mathrm{mmol})$ in dry DCM was stirred at $-78^{\circ} \mathrm{C}$, then the ozone generated by the ozone generator was passed into the solution until TLC (petroleum ether/ethyl acetate 10:1) revealed that conversion of the starting material $3 \mathbf{c}$ was completed and the ozone was stopped. Next, the solution was quenched with dimethyl sulphide (6.0 $\mathrm{mL}$ ) and the reaction mixture was adjusted to room temperature and stirred for $12 \mathrm{~h}$. At last, the solvent was evaporated under reduced pressure, and the crude products were purified through preparative thin layer chromatography (petroleum ether/ethyl acetate 10:1) to get desired products 4 as white solid.

(R)-2-(2-(4-ethylphenyl)-2-oxoethyl)-2,3-dihydronaphthalene-1,4-dione (4)<smiles>CCc1ccc(C(=O)CC2C(=O)c3ccccc3C2=O)cc1</smiles>

White solid (instable); $56.3 \mathrm{mg}, 92 \%$ yield; $\mathrm{mp}: 111-113{ }^{\circ} \mathrm{C} ;[\alpha]_{\mathrm{D}}{ }^{20}=-192.2\left(\mathrm{c}=0.116\right.$, acetone); ${ }^{1} \mathrm{H}$ NMR (400 MHz, $\left.\mathrm{CDCl}_{3}\right)(\delta, \mathrm{ppm}): 8.11-8.08(\mathrm{~m}, 2 \mathrm{H}), 7.97(\mathrm{~d}, J=8.0 \mathrm{~Hz}, 2 \mathrm{H}), 7.79-7.76(\mathrm{~m}, 2 \mathrm{H}), 7.34(\mathrm{~d}, J=8.0 \mathrm{~Hz}, 2 \mathrm{H}), 3.90-3.72(\mathrm{~m}$, 2H), 3.30-3.05 (m, 3H), $2.75(\mathrm{q}, J=7.6 \mathrm{~Hz}, 2 \mathrm{H}), 1.28(\mathrm{~d}, J=7.6 \mathrm{~Hz}, 3 \mathrm{H}) .{ }^{13} \mathrm{C}$ NMR $\left(100 \mathrm{MHz}, \mathrm{CDCl}_{3}\right)(\delta, \mathrm{ppm}): 197.2$, 196.7, 195.4, 150.5, 135.4, 135.3, 134.3, 134.1, 128.4, 128.2, 127.0, 126.6, 43.4, 43.0, 38.4, 29.0, 15.2. IR (KBr, $v, \mathrm{~cm}^{-}$ $\left.{ }^{1}\right)$ : 2966, 2928, 1752, 1685, 1605, 1560, 1413, 828, 780; HRMS (ESI) m/z calcd for $\mathrm{C}_{20} \mathrm{H}_{18} \mathrm{NaO}_{3}[\mathrm{M}+\mathrm{Na}]^{+} 329.1154$, found 329.1156; HPLC: 98\% ee (Daicel Chiralcel OD-H, hexane/i-PrOH =70/30, flow rate: $0.6 \mathrm{~mL} / \mathrm{min}$, detector: 254 $\mathrm{nm}, \mathrm{t}_{\mathrm{R}}($ major $)=12.515 \mathrm{~min}, \mathrm{t}_{\mathrm{R}}($ minor $)=19.747 \mathrm{~min}$.

\section{General procedure for the synthesis of compounds 3}

Example for the synthesis of $\mathbf{3 a}$ :
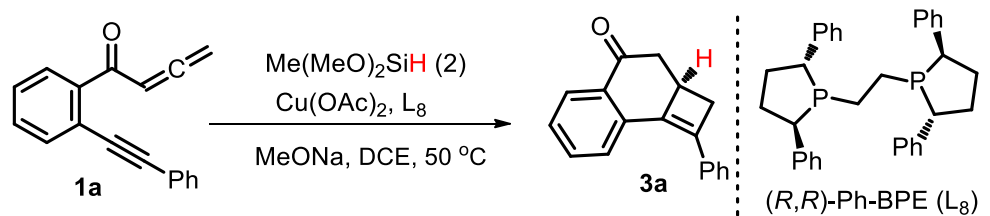

In an Ar-filled glove box, $\mathrm{Cu}(\mathrm{OAc})_{2}(10 \mathrm{~mol} \%),(R, R)-\mathrm{Ph}-\mathrm{BPE}(10 \mathrm{~mol} \%), \mathrm{MeONa}(1.5$ equiv.), 1-(2(phenylethynyl)phenyl)buta-2,3-dien-1-one (1a, $0.2 \mathrm{mmol})$ and DCE $(2.0 \mathrm{~mL})$ were added to a Schlenk tube. The resulting mixture was stirred for 20 minutes at room temperature and then $\mathrm{Me}(\mathrm{MeO})_{2} \mathrm{SiH}(0.6 \mathrm{mmol})$ were added successively. The Schlenk tube was removed from the dry box and the mixture was stirred in the oil bath at $50{ }^{\circ} \mathrm{C}$ for 12 hours. The mixture was concentrated under vacuum and the crude product was purified by column chromatography on silica with a mixture of ethyl acetate/petroleum ether (1:50) to get chiral desired products $\mathbf{3 a}$.

\section{(R)-1-phenyl-2a,3-dihydrocyclobuta[a]naphthalen-4(2H)-one (3a)}

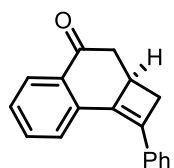


Yellow oil after purification by column chromatography (petroleum ether/ethyl acetate $=50 / 1$ ); $36.9 \mathrm{mg}$, 75\% yield; $[\alpha]_{\mathrm{D}}{ }^{20}=+69.9\left(\mathrm{c}=0.256\right.$, acetone); ${ }^{1} \mathrm{H}$ NMR $\left(400 \mathrm{MHz}, \mathrm{CDCl}_{3}\right)(\delta, \mathrm{ppm}): 8.11-8.09(\mathrm{~m}, 1 \mathrm{H}), 7.81(\mathrm{~d}, J=7.6 \mathrm{~Hz}, 1 \mathrm{H})$, 7.66-7.56 (m, 3H), 7.44-7.31 (m, 4H), 3.29-3.22 (m, 2H), 3.20-3.16 (m, 1H), 2.72-7.65 (m, 2H). ${ }^{13} \mathrm{C}$ NMR (100 MHz, $\left.\mathrm{CDCl}_{3}\right)(\delta, \mathrm{ppm}): 198.0,137.5,136.7,136.4,135.2,133.6,132.5,128.7,128.5,128.2,128.1,126.0,125.0,46.1,35.9$, 35.8. IR ( $\left.\mathrm{KBr}, v, \mathrm{~cm}^{-1}\right)$ : 2949, 2922, $1685,1602,1529,1451,829,728$; HRMS (ESI) $\mathrm{m} / \mathrm{z}$ calcd for $\mathrm{C}_{18} \mathrm{H}_{14} \mathrm{NaO}[\mathrm{M}+\mathrm{Na}]^{+}$ 269.0942, found 269.0944; HPLC: >99\% ee (Daicel Chiralpak OD-H, hexane/i-PrOH =90/10, flow rate: $0.6 \mathrm{~mL} / \mathrm{min}$, detector: $254 \mathrm{~nm}, \mathrm{t}_{\mathrm{R}}($ major$)=11.412 \mathrm{~min}$.

\section{(R)-1-(p-tolyl)-2a,3-dihydrocyclobuta[a]naphthalen-4(2H)-one (3b)}

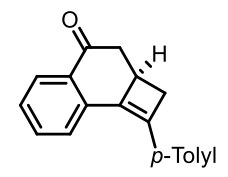

Yellow oil after purification by column chromatography (petroleum ether/ethyl acetate $=50 / 1$ ); $32.2 \mathrm{mg}$, $62 \%$ yield; $[\alpha]_{\mathrm{D}}^{20}=+629.5\left(\mathrm{c}=0.156\right.$, acetone); ${ }^{1} \mathrm{H}$ NMR $\left(400 \mathrm{MHz}, \mathrm{CDCl}_{3}\right)(\delta, \mathrm{ppm}): 8.12-8.10(\mathrm{~m}, 1 \mathrm{H}), 7.81(\mathrm{~d}, J=7.6 \mathrm{~Hz}, 1 \mathrm{H})$, 7.62-7.53 (m, 3H), 7.42-7.35 (m, 1H), $7.23(\mathrm{~d}, J=7.6 \mathrm{~Hz}, 2 \mathrm{H}), 3.33-3.14(\mathrm{~m}, 3 \mathrm{H}), 2.73-2.66(\mathrm{~m}, 2 \mathrm{H}), 2.41(\mathrm{~s}, 3 \mathrm{H}) .{ }^{13} \mathrm{C}$ NMR (100 MHz, $\left.\mathrm{CDCl}_{3}\right)(\delta, \mathrm{ppm}):$ 197.9, 138.5, 137.5, 136.8, 135.1, 133.5, 132.4, 132.4, 129.3, 128.1, 127.8, 125.9, 124.9, 46.1, 35.8, 35.7, 21.4. IR (KBr, $\left.v, \mathrm{~cm}^{-1}\right)$ : 3005, 2909, 1683, 1592, 1510, 1461, 815, 750; HRMS (ESI) m/z calcd for $\mathrm{C}_{19} \mathrm{H}_{16} \mathrm{NaO}[\mathrm{M}+\mathrm{Na}]^{+} 283.1099$, found 283.1095; HPLC: $>99 \%$ ee (Daicel Chiralpak IA, hexane/i-PrOH =99/1, flow rate: $0.6 \mathrm{~mL} / \mathrm{min}$, detector: $254 \mathrm{~nm}, \mathrm{t}_{\mathrm{R}}($ minor $)=13.340 \mathrm{~min}, \mathrm{t}_{\mathrm{R}}($ major $)=15.718 \mathrm{~min}$.

\section{(R)-1-(4-ethylphenyl)-2a,3-dihydrocyclobuta[a]naphthalen-4(2H)-one (3c)}

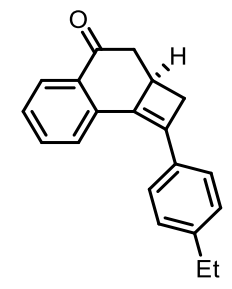

White solid after purification by column chromatography (petroleum ether/ethyl acetate $=50 / 1$ ); $39.7 \mathrm{mg}$, $66 \%$ yield; mp: $140-142{ }^{\circ} \mathrm{C} ;[\alpha]_{\mathrm{D}}{ }^{20}=-1008.8\left(\mathrm{c}=0.26\right.$, acetone); ${ }^{1} \mathrm{H}$ NMR $\left(400 \mathrm{MHz}, \mathrm{CDCl}_{3}\right)(\delta, \mathrm{ppm}): 8.11-8.09(\mathrm{~m}, 1 \mathrm{H}), 7.82(\mathrm{~d}$, $J=7.6 \mathrm{~Hz}, 1 \mathrm{H}), 7.65-7.54(\mathrm{~m}, 3 \mathrm{H}), 7.42-7.36(\mathrm{~m}, 1 \mathrm{H}), 7.26(\mathrm{~d}, J=8.4 \mathrm{~Hz}, 2 \mathrm{H}), 3.32-3.15(\mathrm{~m}, 3 \mathrm{H}), 2.74-2.64(\mathrm{~m}, 4 \mathrm{H})$, $1.28(\mathrm{t}, J=7.6 \mathrm{~Hz}, 3 \mathrm{H}) .{ }^{13} \mathrm{C} \mathrm{NMR}\left(100 \mathrm{MHz}, \mathrm{CDCl}_{3}\right)(\delta, \mathrm{ppm}): 197.9,144.9,137.5,136.8,135.2,133.4,132.6,132.4$, 128.1, 128.0, 127.8, 126.0, 124.9, 46.1, 35.8, 35.7, 28.8, 15.5. IR (KBr, $\left.v, \mathrm{~cm}^{-1}\right): 3005,2965,1683,1594,1507,1462$, 831, 765; HRMS (ESI) $\mathrm{m} / \mathrm{z}$ calcd for $\mathrm{C}_{20} \mathrm{H}_{19} \mathrm{O}[\mathrm{M}+\mathrm{H}]^{+}$275.1436, found 275.1437; HPLC: $>99 \%$ ee (Daicel Chiralpak $\mathrm{AD}-\mathrm{H}$, hexane/i-PrOH $=90 / 10$, flow rate: $0.6 \mathrm{~mL} / \mathrm{min}$, detector: $254 \mathrm{~nm}, \mathrm{t}_{\mathrm{R}}($ minor $)=7.741 \mathrm{~min}, \mathrm{t}_{\mathrm{R}}($ major $)=8.550 \mathrm{~min}$.

(R)-1-(4-(tert-butyl)phenyl)-2a,3-dihydrocyclobuta[a]naphthalen-4(2H)-one (3d)

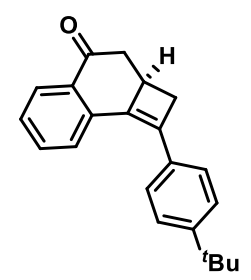

White solid after purification by column chromatography (petroleum ether/ethyl acetate $=50 / 1$ ); $38.7 \mathrm{mg}, 64 \%$ yield; mp: $118-120{ }^{\circ} \mathrm{C} ;[\alpha]_{\mathrm{D}}{ }^{20}=+632.1(\mathrm{c}=0.468$, acetone $) ;{ }^{1} \mathrm{H}$ NMR $\left(400 \mathrm{MHz}, \mathrm{CDCl}_{3}\right)(\delta, \mathrm{ppm}): 8.10-8.08(\mathrm{~m}, 1 \mathrm{H}), 7.82(\mathrm{~d}$, $J=7.6 \mathrm{~Hz}, 1 \mathrm{H}), 7.61-7.57(\mathrm{~m}, 3 \mathrm{H}), 7.48-7.41(\mathrm{~m}, 2 \mathrm{H}), 7.40-7.33(\mathrm{~m}, 1 \mathrm{H}), 3.29-3.14(\mathrm{~m}, 3 \mathrm{H}), 2.71-2.61(\mathrm{~m}, 2 \mathrm{H}), 1.36(\mathrm{~s}$, 
9H). ${ }^{13} \mathrm{C}$ NMR (100 MHz, $\left.\mathrm{CDCl}_{3}\right)(\delta, \mathrm{ppm}): 197.9,151.7,137.4,136.8,135.4,133.4,132.4,128.0,127.8,125.8,125.5$, 124.9, 46.1, 35.8, 35.7, 34.8, 31.2. IR (KBr, $\left.v, \mathrm{~cm}^{-1}\right)$ : 3005, 2961, 1685, 1594, 1507, 1462, 3, 833, 750; HRMS (ESI) $\mathrm{m} / \mathrm{z}$ calcd for $\mathrm{C}_{22} \mathrm{H}_{23} \mathrm{O}[\mathrm{M}+\mathrm{H}]^{+} 303.1749$, found 303.1751; HPLC: >99\% ee (Daicel Chiralcel OD-H, hexane/i-PrOH $=99 / 1$, flow rate: $0.6 \mathrm{~mL} / \mathrm{min}$, detector: $254 \mathrm{~nm}, \mathrm{t}_{\mathrm{R}}($ major $)=17.509 \mathrm{~min}, \mathrm{t}_{\mathrm{R}}($ minor $)=26.991 \mathrm{~min}$.

(R)-1-(4-methoxyphenyl)-2a,3-di-hydrocyclobuta[a]naphthalen-4(2H)-one (3e)

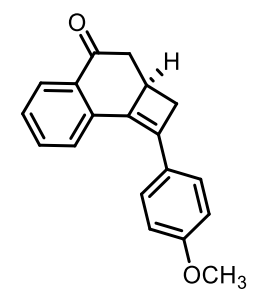

Yellow oil after purification by column chromatography (petroleum ether/ethyl acetate $=50 / 1$ ); $32.6 \mathrm{mg}$, 59\% yield; $[\alpha]_{\mathrm{D}}^{20}=+709.5(\mathrm{c}=0.2$, acetone $) ;{ }^{1} \mathrm{H} \mathrm{NMR}\left(400 \mathrm{MHz}, \mathrm{CDCl}_{3}\right)(\delta, \mathrm{ppm}): 8.09-8.07(\mathrm{~m}, 1 \mathrm{H}), 7.77(\mathrm{~d}, J=7.6 \mathrm{~Hz}, 1 \mathrm{H})$, 7.64-7.49 (m, 3H), 7.42-7.31 (m, 1H), 7.01-6.86 (m, 2H), $3.85(\mathrm{~s}, 3 \mathrm{H}), 3.30-3.12(\mathrm{~m}, 3 \mathrm{H}), 2.72-2.58(\mathrm{~m}, 2 \mathrm{H}) .{ }^{13} \mathrm{C} \mathrm{NMR}$ $\left(100 \mathrm{MHz}, \mathrm{CDCl}_{3}\right)(\delta, \mathrm{ppm}): 198.0,159.8,137.2,137.0,133.7,133.5,132.3,128.1,127.6,127.4,124.7,114.0,55.3,46.1$, 35.7, 35.6. HRMS (ESI) $\mathrm{m} / \mathrm{z}$ calcd for $\mathrm{C}_{19} \mathrm{H}_{17} \mathrm{O}_{2}[\mathrm{M}+\mathrm{H}]^{+} 277.1229$, found 277.1231; HPLC: >99\% ee (Daicel Chiralcel OD-H, hexane/i-PrOH =99/1, flow rate: $1.0 \mathrm{~mL} / \mathrm{min}$, detector: $254 \mathrm{~nm}, \mathrm{t}_{\mathrm{R}}($ minor $)=13.628 \mathrm{~min}, \mathrm{t}_{\mathrm{R}}(\mathrm{major})=18.175 \mathrm{~min}$.

\section{(R)-1-(4-fluorophenyl)-2a,3-dihydrocyclobuta[a]naphthalen-4(2H)-one (3f)}

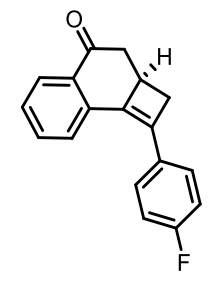

Yellow oil after purification by column chromatography (petroleum ether/ethyl acetate = 50/1); 36.6 mg, 69\% yield; $[\alpha]_{\mathrm{D}}{ }^{20}=+201.6(\mathrm{c}=0.127$, acetone $) ;{ }^{1} \mathrm{H} \mathrm{NMR}\left(400 \mathrm{MHz}, \mathrm{CDCl}_{3}\right)(\delta, \mathrm{ppm}): 8.11-8.09(\mathrm{~m}, 1 \mathrm{H}), 7.75(\mathrm{~d}, J=7.6 \mathrm{~Hz}, 1 \mathrm{H})$, 7.63-7.57 (m, 3H), 7.44-7.34 (m, 1H), 7.15-7.04 (m, 2H), 3.31-3.09 (m, 3H), 2.72-2.61 (m, 2H). ${ }^{13} \mathrm{C} \mathrm{NMR} \mathrm{(100} \mathrm{MHz,}$ $\left.\mathrm{CDCl}_{3}\right)(\delta, \mathrm{ppm}): 197.7,163.9,161.4\left({ }^{1} J_{\mathrm{CF}}=247.4 \mathrm{~Hz}\right), 136.5,136.2,135.7(1), 135.7(9), 133.6,132.4,131.4(2), 131.4(8)$ $\left({ }^{4} J_{\mathrm{CF}}=3.7 \mathrm{~Hz}\right), 128.2,128.1,127.8,127.7\left({ }^{3} J_{\mathrm{CF}}=8.2 \mathrm{~Hz}\right), 124.6,115.7,115.5\left({ }^{2} J_{\mathrm{CF}}=21.5 \mathrm{~Hz}\right), 46.0,35.8,35.8 . \mathrm{IR}(\mathrm{KBr}$, $\left.v, \mathrm{~cm}^{-1}\right): 2980,2865,1700,1636,1454,823,754$; HRMS (ESI) m/z calcd for $\mathrm{C}_{18} \mathrm{H}_{14} \mathrm{FO}[\mathrm{M}+\mathrm{H}]^{+} 265.1029$, found 265.1026; HPLC: >99\% ee (Daicel Chiralpak IC, hexane/i-PrOH =70/30, flow rate: $0.6 \mathrm{~mL} / \mathrm{min}$, detector: $254 \mathrm{~nm}$, $\mathrm{t}_{\mathrm{R}}($ major $)=11.156 \mathrm{~min}, \mathrm{t}_{\mathrm{R}}($ minor $)=11.846 \mathrm{~min}$.

\section{(R)-1-(4-chlorophenyl)-2a,3-dihydrocyclobuta[a]naphthalen-4(2H)-one (3g)}

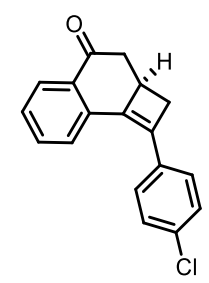

Yellow oil after purification by column chromatography (petroleum ether/ethyl acetate = 50/1); $45.7 \mathrm{mg}, 72 \%$ yield; $[\alpha]_{\mathrm{D}}{ }^{20}=+540.8\left(\mathrm{c}=0.279\right.$, acetone); ${ }^{1} \mathrm{H} \mathrm{NMR}\left(400 \mathrm{MHz}, \mathrm{CDCl}_{3}\right)(\delta, \mathrm{ppm}): 8.11-8.09(\mathrm{~m}, 1 \mathrm{H}), 7.81(\mathrm{~d}, J=7.6 \mathrm{~Hz}, 1 \mathrm{H})$, 7.66-7.56 (m, 3H), 7.44-7.31 (m, 4H), 3.29-3.22 (m, 2H), 3.26-3.16 (m, 1H), 2.71-2.63 (m, 2H). ${ }^{13} \mathrm{C} \mathrm{NMR} \mathrm{(100} \mathrm{MHz,}$ 
$\left.\mathrm{CDCl}_{3}\right)(\delta, \mathrm{ppm}): 197.6,137.0,136.3,136.1,134.1,133.6,133.5,132.5,128.8,128.2,128.2,127.2,124.7,45.9,35.9$, 35.1. IR $\left(\mathrm{KBr}, v, \mathrm{~cm}^{-1}\right)$ : 2940, 2911, 1683, 1591, 1477, 1407, 855, 763; HRMS (ESI) m/z calcd for $\mathrm{C}_{18} \mathrm{H}_{14} \mathrm{ClO}[\mathrm{M}+\mathrm{H}]^{+}$ 281.0733, found 281.0733; HPLC: $96 \%$ ee (Daicel Chiralpak IC, hexane/i-PrOH =90/10, flow rate: $0.6 \mathrm{~mL} / \mathrm{min}$, detector: $254 \mathrm{~nm}, \mathrm{t}_{\mathrm{R}}($ major $)=15.755 \mathrm{~min}, \mathrm{t}_{\mathrm{R}}($ minor $)=17.453 \mathrm{~min}$.

(R)-1-(2-chlorophenyl)-2a,3-dihydrocyclobuta[a]naphthalen-4(2H)-one (3h)

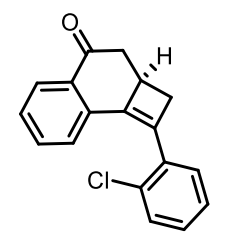

Yellow oil after purification by column chromatography (petroleum ether/ethyl acetate $=50 / 1$ ); $25.3 \mathrm{mg}$, $45 \%$ yield; $[\alpha]_{\mathrm{D}}{ }^{20}=+228.3(\mathrm{c}=0.12$, acetone $) ;{ }^{1} \mathrm{H}$ NMR $\left(400 \mathrm{MHz}, \mathrm{CDCl}_{3}\right)(\delta, \mathrm{ppm}): 8.06(\mathrm{~d}, J=7.6 \mathrm{~Hz}, 1 \mathrm{H}), 7.68-7.61(\mathrm{~m}, 1 \mathrm{H})$, 7.49-7.47 (m, 2H), 7.44-7.33 (m, 2H), 7.27-7.21 (m, 2H), 3.50-3.45 (m, 1H), 3.33-3.24 (m, 1H), 3.20-3.15 (m, 1H), 2.90$2.86(\mathrm{~m}, 1 \mathrm{H}), 2.75-2.68(\mathrm{~m}, 1 \mathrm{H}) .{ }^{13} \mathrm{C}$ NMR $\left(100 \mathrm{MHz}, \mathrm{CDCl}_{3}\right)(\delta, \mathrm{ppm}): 197.7,139.9,135.9,135.8,133.9,133.3,132.8$, 132.4, 130.4, 129.2(2), 129.2(0), 128.3, 127.9, 126.5, 124.8, 45.9, 39.7, 37.0. IR (KBr, $\left.v, \mathrm{~cm}^{-1}\right): 3005,2988,1682,1594$, 1474, 1430, 896, 750; HRMS (ESI) m/z calcd for $\mathrm{C}_{18} \mathrm{H}_{14} \mathrm{ClO}[\mathrm{M}+\mathrm{H}]^{+} 281.0733$, found 281.0732; HPLC: >99\% ee (Daicel Chiralpak AS-H, hexane $/ \mathrm{i}-\mathrm{PrOH}=95 / 5$, flow rate: $0.6 \mathrm{~mL} / \mathrm{min}$, detector: $254 \mathrm{~nm}, \mathrm{t}_{\mathrm{R}}($ minor $)=9.556 \mathrm{~min}$, $\mathrm{t}_{\mathrm{R}}($ major $)=$ 10.219 min.

(R)-1-(3-chlorophenyl)-2a,3-dihydrocyclobuta[a]naphthalen-4(2H)-one (3i)

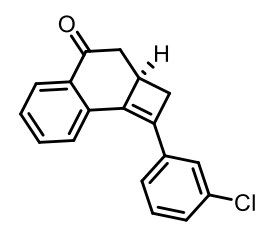

White solid after purification by column chromatography (petroleum ether/ethyl acetate $=50 / 1$ ); $38.6 \mathrm{mg}, 63 \% \mathrm{yield}$; mp: $80-82{ }^{\circ} \mathrm{C} ;[\alpha]_{\mathrm{D}}{ }^{20}=+492.5\left(\mathrm{c}=0.518\right.$, acetone); ${ }^{1} \mathrm{H}$ NMR $\left(400 \mathrm{MHz}, \mathrm{CDCl}_{3}\right)(\delta, \mathrm{ppm}): 8.11-8.09(\mathrm{~m}, 1 \mathrm{H}), 7.75(\mathrm{~d}, J$ $=7.6 \mathrm{~Hz}, 1 \mathrm{H}), 7.63-7.59(\mathrm{~m}, 1 \mathrm{H}), 7.57-7.56(\mathrm{~m}, 1 \mathrm{H}), 7.53-7.51(\mathrm{~m}, 1 \mathrm{H}), 7.42-7.38(\mathrm{~m}, 1 \mathrm{H}), 7.36-7.27(\mathrm{~m}, 2 \mathrm{H}), 3.27-3.15$ (m, 3H), 2.71-2.61 (m, 2H). $\left.{ }^{13} \mathrm{C} \mathrm{NMR} \mathrm{(100} \mathrm{MHz,} \mathrm{CDCl}_{3}\right)(\delta, \mathrm{ppm}): 197.4,138.0,136.8,136.1,135.8,134.7,133.6,132.5$, 129.8, 128.4, 128.3, 128.2, 126.0, 124.8, 124.0, 45.9, 36.0, 35.7. IR (KBr, v, cm $\left.\mathrm{cm}^{-1}\right)$ : 2975, 2922, 1682, 1507, 1456, 1417, 762, 700; HRMS (ESI) m/z calcd for $\mathrm{C}_{18} \mathrm{H}_{14} \mathrm{ClO}[\mathrm{M}+\mathrm{H}]^{+}$281.0733, found 281.0725; HPLC: >99\% ee (Daicel Chiralcel OD-H, hexane/i-PrOH =95/5, flow rate: $0.6 \mathrm{~mL} / \mathrm{min}$, detector: $254 \mathrm{~nm}, \mathrm{t}_{\mathrm{R}}($ minor $)=11.725 \mathrm{~min}, \mathrm{t}_{\mathrm{R}}($ major $)=13.959 \mathrm{~min}$.

\section{(R)-1-(4-bromophenyl)-2a,3-dihydrocyclobuta[a]naphthalen-4(2H)-one (3j)}

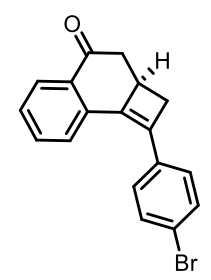

White solid after purification by column chromatography (petroleum ether/ethyl acetate $=50 / 1$ ); $32.4 \mathrm{mg}, 50 \%$ yield; mp: $95-97{ }^{\circ} \mathrm{C} ;[\alpha]_{\mathrm{D}}^{20}=+520.5\left(\mathrm{c}=0.336\right.$, acetone) ${ }^{1} \mathrm{H}$ NMR $\left(400 \mathrm{MHz}, \mathrm{CDCl}_{3}\right)(\delta, \mathrm{ppm}): 8.11-8.08(\mathrm{~m}, 1 \mathrm{H}), 7.73(\mathrm{~d}, J$ $=7.6 \mathrm{~Hz}, 1 \mathrm{H}), 7.61-7.57(\mathrm{~m}, 1 \mathrm{H}), 7.56-7.45(\mathrm{~m}, 4 \mathrm{H}), 7.43-7.36(\mathrm{~m}, 1 \mathrm{H}), 3.25-3.15(\mathrm{~m}, 3 \mathrm{H}), 2.72-2.61(\mathrm{~m}, 2 \mathrm{H}) .{ }^{13} \mathrm{C} \mathrm{NMR}$ $\left(100 \mathrm{MHz}, \mathrm{CDCl}_{3}\right)(\delta, \mathrm{ppm}): 197.5,137.2,136.3,136.1,133.9,133.6,132.5,131.8,128.3,128.2,127.4,124.7,122.3$, 45.9, 35.9, 35.7. IR (KBr, $\left.v, \mathrm{~cm}^{-1}\right): 3061,2908,1682,1594,1486,1396,820,750$; HRMS (ESI) m/z calcd for $\mathrm{C}_{18} \mathrm{H}_{14} \mathrm{BrO}$ 
$[\mathrm{M}+\mathrm{H}]^{+}$325.0228, found 325.0221; HPLC: >99\% ee (Daicel Chiralpak AS-H, hexane/i-PrOH =95/5, flow rate: 0.6 $\mathrm{mL} / \mathrm{min}$, detector: $254 \mathrm{~nm}, \mathrm{t}_{\mathrm{R}}($ minor $)=11.319 \mathrm{~min}, \mathrm{t}_{\mathrm{R}}($ major $)=13.121 \mathrm{~min}$.

\section{(R)-1-(naphthalen-1-yl)-2a,3-dihydrocyclobuta[a]naphthalen-4(2H)-one (3k)}

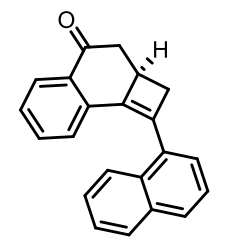

Yellow oil after purification by column chromatography (petroleum ether/ethyl acetate $=50 / 1$ ); 36.1 mg, 61\% yield; $[\alpha]_{\mathrm{D}}{ }^{20}=+1094.3\left(\mathrm{c}=0.12\right.$, acetone); ${ }^{1} \mathrm{H} \mathrm{NMR}\left(400 \mathrm{MHz}, \mathrm{CDCl}_{3}\right)(\delta, \mathrm{ppm}): 8.14-8.12(\mathrm{~m}, 1 \mathrm{H}), 7.96(\mathrm{~s}, 1 \mathrm{H}), 7.92-7.80$ (m, 5H), 7.65-7.60 (m, 1H), 7.55-7.47 (m, 2H), 7.43-7.39 (m, 1H), 3.38-3.18 (m, 3H), 2.79-2.66 (m, $2 \mathrm{H}) .{ }^{13} \mathrm{C} \mathrm{NMR}(100$ $\left.\mathrm{MHz}_{1} \mathrm{CDCl}_{3}\right)(\delta, \mathrm{ppm}): 197.8,137.5,136.8,136.7,133.6,133.4,133.2,132.6,132.5,128.2,128.2,128.2,128.1,127.8$, 126.5, 126.4, 125.2, 124.9, 123.7, 46.1, 36.0, 35.8. IR (KBr, v, $\left.\mathrm{cm}^{-1}\right)$ : 3005, 2989, 1684, 1592, 1458, 1275, 817, 750; HRMS (ESI) $\mathrm{m} / \mathrm{z}$ calcd for $\mathrm{C}_{22} \mathrm{H}_{17} \mathrm{O}[\mathrm{M}+\mathrm{H}]^{+}$297.1279, found 297.1275; HPLC: >99\% ee (Daicel Chiralcel OD-H, hexane/i-PrOH =95/5, flow rate: $0.6 \mathrm{~mL} / \mathrm{min}$, detector: $254 \mathrm{~nm}, \mathrm{t}_{\mathrm{R}}$ (major) $=15.439 \mathrm{~min}$, $\mathrm{t}_{\mathrm{R}}($ minor $)=18.025 \mathrm{~min}$.

(R)-1-(thiophen-2-yl)-2a,3-dihydrocyclobuta[a]naphthalen-4(2H)-one (3l)

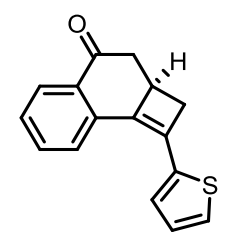

White solid after purification by column chromatography (petroleum ether/ethyl acetate = 50/1); 40.1 mg,70\% yield; mp: $102-104{ }^{\circ} \mathrm{C} ;[\alpha]_{\mathrm{D}}{ }^{20}=+1886.5\left(\mathrm{c}=0.200\right.$, acetone); ${ }^{1} \mathrm{H} \mathrm{NMR}\left(400 \mathrm{MHz}, \mathrm{CDCl}_{3}\right)(\delta, \mathrm{ppm}): 8.07-8.06(\mathrm{~m}, 1 \mathrm{H}), 7.88(\mathrm{~d}, J$ $=7.6 \mathrm{~Hz}, 1 \mathrm{H}), 7.64-7.60(\mathrm{~m}, 1 \mathrm{H}), 7.39-7.33(\mathrm{~m}, 2 \mathrm{H}), 7.20(\mathrm{~d}, J=3.5 \mathrm{~Hz}, 1 \mathrm{H}), 7.08(\mathrm{dd}, J=5.0,3.6 \mathrm{~Hz}, 1 \mathrm{H}), 3.41-3.24$ $(\mathrm{m}, 1 \mathrm{H}), 3.22-3.11(\mathrm{~m}, 2 \mathrm{H}), 2.77-2.66(\mathrm{~m}, 2 \mathrm{H}) .{ }^{13} \mathrm{C} \mathrm{NMR}\left(100 \mathrm{MHz}, \mathrm{CDCl}_{3}\right)(\delta, \mathrm{ppm}): 197.6,138.0,135.9,133.6,133.4$, 132.2, 130.6, 128.0, 127.9, 127.5, 125.9(2), 125.9(9), 125.1, 45.8, 36.4, 36.2. IR (KBr, v, cm $\left.{ }^{-1}\right): 3059,2911,1680,1591$, 1560, 1463, 852, 709; HRMS (ESI) m/z calcd for $\mathrm{C}_{16} \mathrm{H}_{13} \mathrm{OS}[\mathrm{M}+\mathrm{H}]^{+}$253.0687, found 253.0694; HPLC: >99\% ee (Daicel Chiralcel OD-H, hexane/i-PrOH =95/5, flow rate: $0.6 \mathrm{~mL} / \mathrm{min}$, detector: $254 \mathrm{~nm}, \mathrm{t}_{\mathrm{R}}($ minor $)=12.618$ min, $\mathrm{t}_{\mathrm{R}}($ major $)=$ $13.255 \mathrm{~min}$.

\section{(R)-1-(cyclohex-1-en-1-yl)-2a,3-dihydrocyclobuta[a]naphthalen-4(2H)-one (3m)}

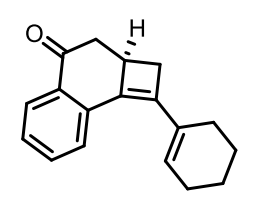

Colorless oil after purification by column chromatography (petroleum ether/ethyl acetate = 50/1); 27.5 mg, 55\% yield; $[\alpha]_{\mathrm{D}}{ }^{20}=+43.9\left(\mathrm{c}=0.13\right.$, acetone); ${ }^{1} \mathrm{H} \mathrm{NMR}(400 \mathrm{MHz}$, Acetone) $(\delta, \mathrm{ppm}): 7.96(\mathrm{~d}, \mathrm{~J}=7.6 \mathrm{~Hz}, 1 \mathrm{H}), 7.74-7.55(\mathrm{~m}, 2 \mathrm{H})$, 7.39-7.36 (m, 1H), $6.00(\mathrm{~s}, 1 \mathrm{H}), 3.13-2.96(\mathrm{~m}, 2 \mathrm{H}), 2.67-2.52(\mathrm{~m}, 2 \mathrm{H}), 2.46-2.34(\mathrm{~m}, 2 \mathrm{H}), 2.21(\mathrm{~s}, 2 \mathrm{H}), 1.82(\mathrm{~s}, 1 \mathrm{H}), 1.65$ $(\mathrm{d}, \mathrm{J}=41.6 \mathrm{~Hz}, 3 \mathrm{H}), 1.30(\mathrm{~s}, 1 \mathrm{H}) .{ }^{13} \mathrm{C} \mathrm{NMR}(100 \mathrm{MHz}$, Acetone) ( $\delta, \mathrm{ppm}): 196.6,139.9,136.8,133.8,133.2,133.1$, 132.2, 128.0, 127.3, 126.0, 45.7, 35.1, 34.2, 27.1, 25.5, 22.3, 21.7. IR (KBr, $\left.v, \mathrm{~cm}^{-1}\right): 3051,2949,1676,1592,1509,1445$, 1423, 839, 760; HRMS (ESI) m/z calcd for $\mathrm{C}_{18} \mathrm{H}_{18} \mathrm{NaO}[\mathrm{M}+\mathrm{Na}]^{+} 273.1255$, found 273.1245; HPLC: $98 \%$ ee (Daicel Chiralcel OJ-H, hexane/i-PrOH $=70 / 30$, flow rate: $0.6 \mathrm{~mL} / \mathrm{min}$, detector: $254 \mathrm{~nm}, \mathrm{t}_{\mathrm{R}}($ minor $)=8.186$ min, $\mathrm{t}_{\mathrm{R}}($ major $)=$ $8.642 \mathrm{~min}$. 


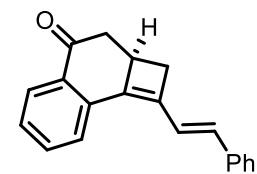

Yellow oil after purification by column chromatography (petroleum ether/ethyl acetate $=50 / 1$ ); $37.5 \mathrm{mg}, 69 \%$ yield; $[\alpha]_{\mathrm{D}}{ }^{20}$ $=+360.9\left(\mathrm{c}=0.041\right.$, acetone); ${ }^{1} \mathrm{H} \mathrm{NMR}\left(400 \mathrm{MHz}, \mathrm{CDCl}_{3}\right)(\delta, \mathrm{ppm}): 8.04(\mathrm{~d}, J=8.0 \mathrm{~Hz}, 1 \mathrm{H}), 7.59-7.50(\mathrm{~m}, 4 \mathrm{H}), 7.41-$ $7.34(\mathrm{~m}, 3 \mathrm{H}), 7.31(\mathrm{~d}, J=7.6 \mathrm{~Hz}, 1 \mathrm{H}), 7.18(\mathrm{~d}, J=16.0 \mathrm{~Hz}, 1 \mathrm{H}), 6.72(\mathrm{~d}, J=15.6 \mathrm{~Hz}, 1 \mathrm{H}), 3.34-3.26(\mathrm{~m}, 1 \mathrm{H}), 3.18-3.09$ $(\mathrm{m}, 2 \mathrm{H}), 2.71-2.64(\mathrm{~m}, 2 \mathrm{H}) .{ }^{13} \mathrm{C} \mathrm{NMR}\left(100 \mathrm{MHz}, \mathrm{CDCl}_{3}\right)(\delta, \mathrm{ppm}): 197.8,137.7,137.3,136.8,136.3,133.6,132.3$, 132.0, 128.7, 128.2, 127.9, 127.7, 126.7, 124.9, 121.5, 45.8, 35.7, 34.7. IR (KBr, v, cm $\left.\mathrm{cm}^{-1}\right): 3035,2957,1678,1623,1567$, 1409, 887, 798; HRMS (ESI) m/z calcd for $\mathrm{C}_{20} \mathrm{H}_{17} \mathrm{O}[\mathrm{M}+\mathrm{H}]^{+}$273.1279, found 273.1272; HPLC: >99\% ee (Daicel Chiralpak IA, hexane $/ \mathrm{i}-\mathrm{PrOH}=95 / 5$, flow rate: $0.6 \mathrm{~mL} / \mathrm{min}$, detector: $254 \mathrm{~nm}, \mathrm{t}_{\mathrm{R}}(\operatorname{minor})=10.639 \mathrm{~min}, \mathrm{t}_{\mathrm{R}}(\operatorname{major})=11.536$ $\min$.

(R)-1-(phenylethynyl)-2a,3-dihydrocyclobuta[a]naphthalen-4(2H)-one (3o)

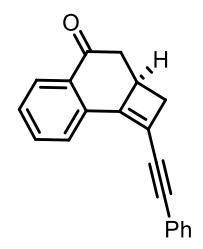

Yellow oil after purification by column chromatography (petroleum ether/ethyl acetate = 50/1);25.4 mg, 47\% yield; $[\alpha]_{\mathrm{D}}{ }^{20}$ $=+286.0\left(\mathrm{c}=0.21\right.$, acetone); ${ }^{1} \mathrm{H} \mathrm{NMR}\left(400 \mathrm{MHz}, \mathrm{CDCl}_{3}\right)(\delta, \mathrm{ppm}): 8.01(\mathrm{~d}, J=7.2 \mathrm{~Hz}, 1 \mathrm{H}), 7.72(\mathrm{~d}, J=7.6 \mathrm{~Hz}, 1 \mathrm{H})$, 7.61-7.57 (m, 1H), 7.54-7.49 (m, 2H), 7.41-7.34 (m, 4H), 3.34-3.25 (m, 1H), 3.16-3.06 (m, 2H), 2.77-2.65 (m, $2 \mathrm{H}) .{ }^{13} \mathrm{C}$ NMR (100 MHz, $\left.\mathrm{CDCl}_{3}\right)(\delta, \mathrm{ppm}):$ 197.2, 147.3, 135.1, 133.8, 132.1, 131.8, 128.7, 128.6, 128.4, 127.6, 126.7, 124.5, 122.8, 117.8, 93.9, 84.7, 45.3, 39.3, 36.9. IR (KBr, $\left.v, \mathrm{~cm}^{-1}\right): 3065,2936,1640,1609,1554,1439,847,754$; HRMS (ESI) $\mathrm{m} / \mathrm{z}$ calcd for $\mathrm{C}_{20} \mathrm{H}_{14} \mathrm{NaO}[\mathrm{M}+\mathrm{Na}]^{+}$293.0942, found 293.0934; HPLC: 72\% ee (Daicel Chiralcel OD-H, hexane/i-PrOH $=99 / 1$, flow rate: $0.6 \mathrm{~mL} / \mathrm{min}$, detector: $254 \mathrm{~nm}, \mathrm{t}_{\mathrm{R}}($ minor $)=22.919 \mathrm{~min}, \mathrm{t}_{\mathrm{R}}($ major $)=27.665 \mathrm{~min}$.

\section{(R)-7-methyl-1-(p-tolyl)-2a,3-dihydrocyclobuta[a]naphthalen-4(2H)-one (3q)}

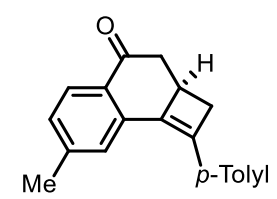

White solid after purification by column chromatography (petroleum ether/ethyl acetate $=50 / 1$ ); $41.8 \mathrm{mg}, 76 \% \mathrm{yield}$; mp: $114-116{ }^{\circ} \mathrm{C} ;[\alpha]_{\mathrm{D}}^{20}=+439.8\left(\mathrm{c}=0.728\right.$, acetone); ${ }^{1} \mathrm{H}$ NMR $\left(400 \mathrm{MHz}, \mathrm{CDCl}_{3}\right)(\delta, \mathrm{ppm}): 7.99(\mathrm{~d}, J=8.0 \mathrm{~Hz}, 1 \mathrm{H})$, 7.60-7.51 (m, 3H), 7.26-7.14 (m, 3H), 3.24-3.09 (m, 3H), 2.67-2.60 (m, 2H), 2.45 (s, 3H), 2.40 (s, 3H). ${ }^{13} \mathrm{C} \mathrm{NMR} \mathrm{(100}$ $\left.\mathrm{MHz}, \mathrm{CDCl}_{3}\right)(\delta, \mathrm{ppm}): 197.7,144.2,138.4,137.2,136.9,135.4,132.5,130.2,129.3,128.8,128.1,125.9,125.2,46.0$, 35.9, 35.7, 22.0, 21.4. IR (KBr, $\left.v, \mathrm{~cm}^{-1}\right)$ : 2989, 2931, 1683, 1600, 1510, 1467, 829, 747; HRMS (ESI) m/z calcd for $\mathrm{C}_{20} \mathrm{H}_{19} \mathrm{O}[\mathrm{M}+\mathrm{H}]^{+}$275.1436, found 275.1432; HPLC: >99\% ee (Daicel Chiralcel OD-H, hexane/i-PrOH =99/1, flow rate: $0.6 \mathrm{~mL} / \mathrm{min}$, detector: $254 \mathrm{~nm}, \mathrm{t}_{\mathrm{R}}($ minor $)=13.817 \mathrm{~min}, \mathrm{t}_{\mathrm{R}}($ major $)=18.564 \mathrm{~min}$.

\section{(R)-1-(4-methoxyphenyl)-7-methyl-2a,3-dihydrocyclobuta[a]naphthalen-4(2H)-one (3r)}




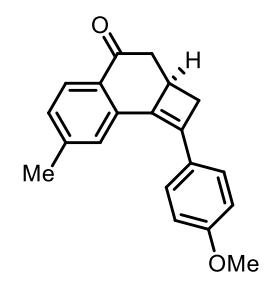

White solid after purification by column chromatography (petroleum ether/ethyl acetate = 50/1); $32.0 \mathrm{mg}$, 55\% yield; mp: $118-120{ }^{\circ} \mathrm{C} ;[\alpha]_{\mathrm{D}}^{20}=+647.1\left(\mathrm{c}=0.384\right.$, acetone); ${ }^{1} \mathrm{H} \mathrm{NMR}\left(400 \mathrm{MHz}, \mathrm{CDCl}_{3}\right)(\delta, \mathrm{ppm}): 7.98(\mathrm{~d}, J=8.0 \mathrm{~Hz}, 1 \mathrm{H})$, 7.59-7.55 (m, 3H), 7.17-7.15 (m, 1H), 6.97-6.91 (m, 2H), 3.85 (s, 3H), 3.22-3.09 (m, 3H), 2.67-2.58 (m, $2 \mathrm{H}), 2.45(\mathrm{~s}$, $3 \mathrm{H}) .{ }^{13} \mathrm{C} \mathrm{NMR}\left(100 \mathrm{MHz}, \mathrm{CDCl}_{3}\right)(\delta, \mathrm{ppm}): 197.8,159.7,144.2,137.0,136.9,133.9,130.1,128.6,128.2,128.2,127.4$, 125.0, 114.0, 55.3, 46.0, 35.7, 35.7, 22.0. IR (KBr, $\left.v, \mathrm{~cm}^{-1}\right): 3004,2907,1675,1606,1510,1463,828$, 764; HRMS (ESI) $\mathrm{m} / \mathrm{z}$ calcd for $\mathrm{C}_{20} \mathrm{H}_{19} \mathrm{O}_{2}[\mathrm{M}+\mathrm{H}]^{+}$291.1385, found 291.1381; HPLC: >99\% ee (Daicel Chiralcel OD-H, hexane/i-PrOH $=95 / 5$, flow rate: $0.6 \mathrm{~mL} / \mathrm{min}$, detector: $254 \mathrm{~nm}, \mathrm{t}_{\mathrm{R}}($ minor $)=12.597 \mathrm{~min}, \mathrm{t}_{\mathrm{R}}($ major $)=15.511 \mathrm{~min}$.

(R)-1-(4-chlorophenyl)-7-methyl-2a,3-dihydrocyclobuta[a]naphthalen-4(2H)-one (3s)

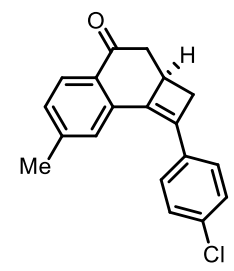

Yellow oil after purification by column chromatography (petroleum ether/ethyl acetate = 50/1); 28.9 mg, $49 \%$ yield; $[\alpha]_{\mathrm{D}}{ }^{20}=+242.3(\mathrm{c}=0.104$, acetone $) ;{ }^{1} \mathrm{H} \mathrm{NMR}\left(400 \mathrm{MHz}, \mathrm{CDCl}_{3}\right)(\delta, \mathrm{ppm}): 8.00(\mathrm{~d}, J=8.0 \mathrm{~Hz}, 1 \mathrm{H}), 7.59-7.50(\mathrm{~m}, 3 \mathrm{H})$, 7.40-7.34 (m, 2H), $7.21(\mathrm{~d}, J=8.0 \mathrm{~Hz}, 1 \mathrm{H}), 3.23-3.12(\mathrm{~m}, 3 \mathrm{H}), 2.69-2.59(\mathrm{~m}, 2 \mathrm{H}), 2.45(\mathrm{~s}, 3 \mathrm{H}) .{ }^{13} \mathrm{C} \mathrm{NMR}(100 \mathrm{MHz}$, $\left.\mathrm{CDCl}_{3}\right)(\delta, \mathrm{ppm}): 197.4,144.4,137.2,136.4,135.8,134.0,133.6,130.3,129.3,128.8,128.3,127.2,125.0,45.9,36.0$, 35.7, 22.0. IR (KBr, $\left.v, \mathrm{~cm}^{-1}\right): 3005,2922,1682,1602,1490,1401,825,746$; HRMS (ESI) m/z calcd for $\mathrm{C}_{19} \mathrm{H}_{16} \mathrm{ClO}$ $[\mathrm{M}+\mathrm{H}]^{+}$295.0890, found 295.0885; HPLC: $99 \%$ ee (Daicel Chiralcel OD-H, hexane/i-PrOH =95/5, flow rate: 0.6 $\mathrm{mL} / \mathrm{min}$, detector: $254 \mathrm{~nm}, \mathrm{t}_{\mathrm{R}}($ minor $)=10.420 \mathrm{~min}, \mathrm{t}_{\mathrm{R}}($ major $)=11.135 \mathrm{~min}$.

\section{(R)-1-(4-chlorophenyl)-7-fluoro-2a,3-dihydrocyclobuta[a]naphthalen-4(2H)-one (3t)}

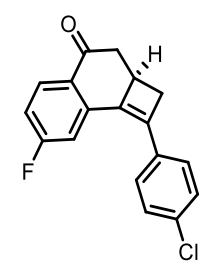

White solid after purification by column chromatography (petroleum ether/ethyl acetate $=50 / 1$ ); $23.8 \mathrm{mg}, 40 \%$ yield; mp: $134-136{ }^{\circ} \mathrm{C} ;[\alpha]_{\mathrm{D}}{ }^{20}=+570.5$ (c = 0.200, acetone); ${ }^{1} \mathrm{H}$ NMR $\left(400 \mathrm{MHz}, \mathrm{CDCl}_{3}\right)(\delta, \mathrm{ppm}): 8.14-8.10(\mathrm{~m}, 1 \mathrm{H}), 7.54-$ $7.50(\mathrm{~m}, 2 \mathrm{H}), 7.41-7.34(\mathrm{~m}, 3 \mathrm{H}), 7.09-7.04(\mathrm{~m}, 1 \mathrm{H}), 3.26-3.12(\mathrm{~m}, 3 \mathrm{H}), 2.69-2.60(\mathrm{~m}, 2 \mathrm{H}) .{ }^{13} \mathrm{C} \mathrm{NMR}(100 \mathrm{MHz}, \mathrm{CDCl} 3)$

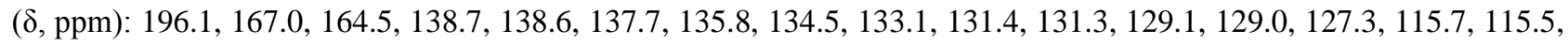
111.3, 111.1, 45.6, 36.0, 35.9. IR (KBr, $\left.v, \mathrm{~cm}^{-1}\right): 3005,2989,1684,1600,1491,1401,828,763$; HRMS (ESI) m/z calcd for $\mathrm{C}_{18} \mathrm{H}_{13} \mathrm{ClFO}[\mathrm{M}+\mathrm{H}]^{+}$299.0639, found 299.0640; HPLC: >99\% ee (Daicel Chiralcel OD-H, hexane/i-PrOH =95/5, flow rate: $0.6 \mathrm{~mL} / \mathrm{min}$, detector: $254 \mathrm{~nm}, \mathrm{t}_{\mathrm{R}}($ minor $)=11.232 \mathrm{~min}, \mathrm{t}_{\mathrm{R}}($ major $)=12.095 \mathrm{~min}$.

(R)-7-chloro-1-(p-tolyl)-2a,3-dihydrocyclobuta[a]naphthalen-4(2H)-one (3u) 


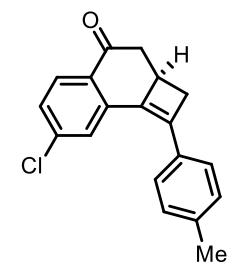

White solid after purification by column chromatography (petroleum ether/ethyl acetate $=50 / 1$ ); $35.3 \mathrm{mg}, 60 \% \mathrm{yield}$; mp: $126-128{ }^{\circ} \mathrm{C} ;[\alpha]_{\mathrm{D}}^{20}=+338.7\left(\mathrm{c}=0.194\right.$, acetone); ${ }^{1} \mathrm{H} \mathrm{NMR}\left(400 \mathrm{MHz}, \mathrm{CDCl}_{3}\right)(\delta, \mathrm{ppm}): 8.01(\mathrm{~d}, J=8.4 \mathrm{~Hz}, 1 \mathrm{H})$, $7.73(\mathrm{~d}, J=2.0 \mathrm{~Hz}, 1 \mathrm{H}), 7.49$ (d, $J=8.0 \mathrm{~Hz}, 2 \mathrm{H}), 7.32-7.30(\mathrm{~m}, 1 \mathrm{H}), 7.24$ (d, $J=8.0 \mathrm{~Hz}, 2 \mathrm{H}), 3.25-3.12(\mathrm{~m}, 3 \mathrm{H}), 2.68$ $2.60(\mathrm{~m}, 2 \mathrm{H}), 2.40$ (s, 3H). ${ }^{13} \mathrm{C} \mathrm{NMR}\left(100 \mathrm{MHz}, \mathrm{CDCl}_{3}\right)(\delta, \mathrm{ppm}): 196.9,139.9,139.2,139.0,138.0,133.6,132.0,130.6$, 129.7, 129.4, 128.0, 126.0, 124.6, 45.8, 35.9, 35.7, 21.5. IR (KBr, v, cm $\left.{ }^{-1}\right): 3005,2985,1685,1586,1511,1416,897$, 764; HRMS (ESI) m/z calcd for $\mathrm{C}_{19} \mathrm{H}_{16} \mathrm{ClO}[\mathrm{M}+\mathrm{H}]^{+}$295.0890, found 295.0891; HPLC: >99\% ee (Daicel Chiralcel OD$\mathrm{H}$, hexane/i-PrOH =95/5, flow rate: $0.6 \mathrm{~mL} / \mathrm{min}$, detector: $254 \mathrm{~nm}, \mathrm{t}_{\mathrm{R}}($ minor $)=9.823 \mathrm{~min}, \mathrm{t}_{\mathrm{R}}($ major $)=10.313 \mathrm{~min}$.

(R)-6-methyl-1-phenyl-2a,3-dihydrocyclobuta[a]naphthalen-4(2H)-one (3v)

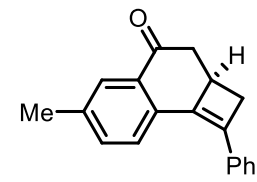

White solid after purification by column chromatography (petroleum ether/ethyl acetate = 50/1); $38.6 \mathrm{mg}, 74 \%$ yield; mp: $72-74{ }^{\circ} \mathrm{C} ;[\alpha]_{\mathrm{D}}{ }^{20}=+444.2\left(\mathrm{c}=0.484\right.$, acetone); ${ }^{1} \mathrm{H}$ NMR $\left(400 \mathrm{MHz}, \mathrm{CDCl}_{3}\right)(\delta, \mathrm{ppm}): 7.91(\mathrm{~d}, J=0.4 \mathrm{~Hz}, 1 \mathrm{H}), 7.71$ $(\mathrm{d}, J=8.0 \mathrm{~Hz}, 1 \mathrm{H}), 7.64-7.62(\mathrm{~m}, 2 \mathrm{H}), 7.42-7.38(\mathrm{~m}, 3 \mathrm{H}), 7.34-7.32(\mathrm{~m}, 1 \mathrm{H}), 3.25-3.14(\mathrm{~m}, 3 \mathrm{H}), 2.69-2.62(\mathrm{~m}, 2 \mathrm{H}), 2.42$ (s, 3H). ${ }^{13} \mathrm{C}$ NMR $\left(100 \mathrm{MHz}, \mathrm{CDCl}_{3}\right)(\delta, \mathrm{ppm}): 198.2,138.1,136.4,136.4,135.2,134.3,134.1,132.3,128.6,128.3,128.2$, 125.9, 124.9, 46.1, 35.9, 35.6, 21.4. IR (KBr, $\left.v, \mathrm{~cm}^{-1}\right): 3005,2989,1684,1605,1493,1474,825,762 ; \mathrm{HRMS}(\mathrm{ESI}) \mathrm{m} / \mathrm{z}$ calcd for $\mathrm{C}_{19} \mathrm{H}_{17} \mathrm{O}[\mathrm{M}+\mathrm{H}]^{+}$261.1279, found 261.1278; HPLC: $>99 \%$ ee (Daicel Chiralcel OD-H, hexane/i-PrOH $=95 / 5$, flow rate: $0.6 \mathrm{~mL} / \mathrm{min}$, detector: $254 \mathrm{~nm}, \mathrm{t}_{\mathrm{R}}($ minor $)=9.906 \mathrm{~min}, \mathrm{t}_{\mathrm{R}}($ major $)=12.111 \mathrm{~min}$.

\section{(R)-6-methoxy-1-phenyl-2a,3-dihydrocyclobuta[a]naphthalen-4(2H)-one (3w)}

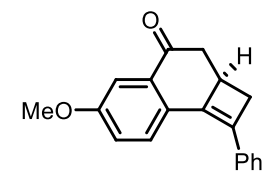

Yellow oil after purification by column chromatography (petroleum ether/ethyl acetate $=50 / 1$ ); $33.8 \mathrm{mg}, 61 \%$ yield; $[\alpha]_{\mathrm{D}}{ }^{20}=-464.1(\mathrm{c}=0.128$, acetone $) ;{ }^{1} \mathrm{H} \mathrm{NMR}\left(400 \mathrm{MHz}, \mathrm{CDCl}_{3}\right)(\delta, \mathrm{ppm}): 7.75(\mathrm{~d}, J=8.4 \mathrm{~Hz}, 1 \mathrm{H}), 7.62-7.59(\mathrm{~m}, 3 \mathrm{H})$, 7.42-7.37 (m, 2H), 7.33-7.27 (m, 1H), 7.17-7.15 (m, 1H), $3.89(\mathrm{~s}, 3 \mathrm{H}), 3.25-3.13(\mathrm{~m}, 3 \mathrm{H}), 2.70-2.61(\mathrm{~m}, 2 \mathrm{H}) .{ }^{13} \mathrm{C} \mathrm{NMR}$ $\left(100 \mathrm{MHz}, \mathrm{CDCl}_{3}\right)(\delta, \mathrm{ppm}): 197.9,159.4,136.1,135.3,135.2,133.8,130.0,128.6,128.1,126.5,125.8,121.4,110.7$,

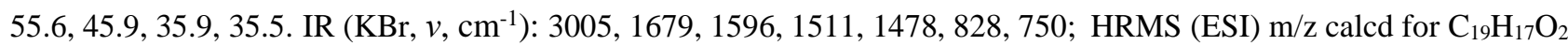
$[\mathrm{M}+\mathrm{H}]^{+}$277.1229, found 277.1231; HPLC: $>99 \%$ ee (Daicel Chiralcel OD-H, hexane/i-PrOH =95/5, flow rate: 0.6 $\mathrm{mL} / \mathrm{min}$, detector: $254 \mathrm{~nm}, \mathrm{t}_{\mathrm{R}}($ minor $)=12.267 \mathrm{~min}, \mathrm{t}_{\mathrm{R}}($ major $)=15.445 \mathrm{~min}$.

(R)-6-methoxy-1-(p-tolyl)-2a,3-dihydrocyclobuta[a]naphthalen-4(2H)-one (3x)

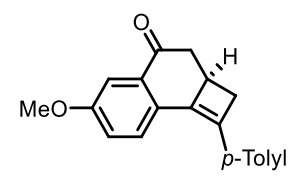


Yellow oil after purification by column chromatography (petroleum ether/ethyl acetate $=50 / 1$ ); $30.7 \mathrm{mg}, 53 \%$ yield; $[\alpha]_{\mathrm{D}}{ }^{20}=-35.8\left(\mathrm{c}=0.226\right.$, acetone); ${ }^{1} \mathrm{H} \mathrm{NMR}\left(400 \mathrm{MHz}, \mathrm{CDCl}_{3}\right)(\delta, \mathrm{ppm}): 7.73(\mathrm{~d}, J=8.4 \mathrm{~Hz}, 1 \mathrm{H}), 7.58(\mathrm{~d}, J=2.8 \mathrm{~Hz}$, $1 \mathrm{H}), 7.50(\mathrm{~d}, J=8.0 \mathrm{~Hz}, 2 \mathrm{H}), 7.20(\mathrm{~d}, J=8.0 \mathrm{~Hz}, 2 \mathrm{H}), 7.21-7.14(\mathrm{~m}, 1 \mathrm{H}), 3.88(\mathrm{~s}, 3 \mathrm{H}), 3.25-3.12(\mathrm{~m}, 3 \mathrm{H}), 2.69-2.62(\mathrm{~m}$, 2H), 2.38 (s, 3H). ${ }^{13} \mathrm{C}$ NMR (100 MHz, $\left.\mathrm{CDCl}_{3}\right)(\delta, \mathrm{ppm}): 198.0,159.2,138.1,135.2,134.9,133.7,132.6,130.2,129.2$, 126.4, 125.7, 121.4, 110.6, 55.6, 46.0, 35.8, 35.5, 21.4. IR (KBr, $\left.v, \mathrm{~cm}^{-1}\right)$ : 3005, 2989, 1680, 1599, 1511, 1478, 813, 750; HRMS (ESI) $\mathrm{m} / \mathrm{z}$ calcd for $\mathrm{C}_{20} \mathrm{H}_{19} \mathrm{O}_{2}[\mathrm{M}+\mathrm{H}]^{+}$291.1385, found 291.1381; HPLC: >99\% ee (Daicel Chiralcel OD-H, hexane $/ \mathrm{i}-\mathrm{PrOH}=95 / 5$, flow rate: $0.6 \mathrm{~mL} / \mathrm{min}$, detector: $254 \mathrm{~nm}, \mathrm{t}_{\mathrm{R}}($ minor $)=10.727 \mathrm{~min}, \mathrm{t}_{\mathrm{R}}($ major $)=12.598 \mathrm{~min}$.

\section{(R)-1-(4-fluorophenyl)-6-methoxy-2a,3-dihydrocyclobuta[a]naphthalen-4(2H)-one (3y)}

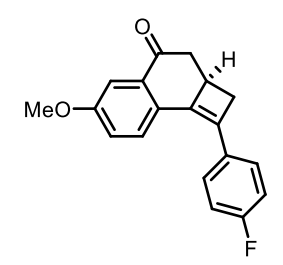

Yellow oil after purification by column chromatography (petroleum ether/ethyl acetate $=50 / 1) ; 42.3 \mathrm{mg}$, 72\% yield; $[\alpha]_{\mathrm{D}}{ }^{20}=-116.3\left(\mathrm{c}=0.086\right.$, acetone); ${ }^{1} \mathrm{H} \mathrm{NMR}\left(400 \mathrm{MHz}, \mathrm{CDCl}_{3}\right)(\delta, \mathrm{ppm}): 7.68(\mathrm{~d}, J=8.8 \mathrm{~Hz}, 1 \mathrm{H}), 7.61-7.54(\mathrm{~m}, 3 \mathrm{H})$, 7.17-7.14 (m, 1H), 7.09-7.05 (m, 2H), $3.89(\mathrm{~s}, 3 \mathrm{H}), 3.23-3.12(\mathrm{~m}, 3 \mathrm{H}), 2.68-2.62(\mathrm{~m}, 2 \mathrm{H}) .{ }^{13} \mathrm{C} \mathrm{NMR}\left(100 \mathrm{MHz}, \mathrm{CDCl}_{3}\right)$ $(\delta, \mathrm{ppm}): 197.7,163.6,161.2\left({ }^{1} J_{\mathrm{CF}}=246.8 \mathrm{~Hz}\right), 159.4,135.5(0), 135.5(8), 133.9(4), 133.9(5)\left({ }^{3} J_{\mathrm{CF}}=8.6 \mathrm{~Hz}\right), 131.7,131.6$ $\left({ }^{4} J_{\mathrm{CF}}=3.3 \mathrm{~Hz}\right), 129.8,127.5,127.4,126.2,121.4,115.7,115.5\left({ }^{2} J_{\mathrm{CF}}=21.5 \mathrm{~Hz}\right), 110.8,55.6,45.9,35.8,35.6 . \mathrm{IR}(\mathrm{KBr}$, $\left.v, \mathrm{~cm}^{-1}\right): 3005,2989,1685,1597,1508,1462,830,763$; HRMS (ESI) m/z calcd for $\mathrm{C}_{19} \mathrm{H}_{15} \mathrm{NaFO}_{2}[\mathrm{M}+\mathrm{Na}]^{+} 317.0954$, found 317.0957; HPLC: >99\% ee (Daicel Chiralcel OD-H, hexane/i-PrOH =95/5, flow rate: $0.6 \mathrm{~mL} / \mathrm{min}$, detector: 254 $\mathrm{nm}, \mathrm{t}_{\mathrm{R}}($ minor $)=11.816 \mathrm{~min}, \mathrm{t}_{\mathrm{R}}($ major $)=12.692 \mathrm{~min}$.

(R)-1-(4-chlorophenyl)-6-methoxy-2a,3-dihydrocyclobuta[a]naphthalen-4(2H)-one (3z)

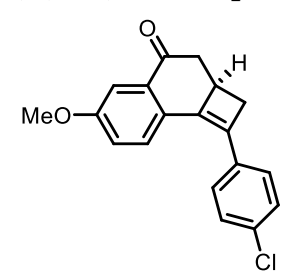

Yellow oil after purification by column chromatography (petroleum ether/ethyl acetate $=50 / 1$ ); $54.6 \mathrm{mg}, 88 \%$ yield; $[\alpha]_{\mathrm{D}}{ }^{20}=-38.4\left(\mathrm{c}=0.700\right.$, acetone); ${ }^{1} \mathrm{H} \mathrm{NMR}\left(400 \mathrm{MHz}, \mathrm{CDCl}_{3}\right)(\delta, \mathrm{ppm}): 7.68(\mathrm{~d}, J=8.4 \mathrm{~Hz}, 1 \mathrm{H}), 7.59(\mathrm{~d}, J=2.8 \mathrm{~Hz}$, $1 \mathrm{H}), 7.54-7.50(\mathrm{~m}, 2 \mathrm{H}), 7.38-7.33(\mathrm{~m}, 2 \mathrm{H}), 7.18-7.15(\mathrm{~m}, 1 \mathrm{H}), 3.89(\mathrm{~s}, 3 \mathrm{H}), 3.24-3.14(\mathrm{~m}, 3 \mathrm{H}), 2.70-2.62(\mathrm{~m}, 2 \mathrm{H}) .{ }^{13} \mathrm{C}$ NMR (100 MHz, $\left.\mathrm{CDCl}_{3}\right)(\delta, \mathrm{ppm}): 197.6,159.6,136.8,133.9,133.8,133.8,133.7,129.6,128.8,127.0,126.3,121.4$, 110.8, 55.6, 45.8, 36.0, 35.4. IR (KBr, $\left.v, \mathrm{~cm}^{-1}\right): 3005,2989,1685,1598,1492,1474,822,766$; HRMS (ESI) m/z calcd for $\mathrm{C}_{19} \mathrm{H}_{15} \mathrm{ClNaO}_{2}[\mathrm{M}+\mathrm{Na}]^{+}$333.0658, found 333.0656; HPLC: >99\% ee (Daicel Chiralcel OD-H, hexane/i-PrOH = 95/5, flow rate: $0.6 \mathrm{~mL} / \mathrm{min}$, detector: $254 \mathrm{~nm}, \mathrm{t}_{\mathrm{R}}($ minor $)=12.583 \mathrm{~min}, \mathrm{t}_{\mathrm{R}}($ major $)=13.148 \mathrm{~min}$.

\section{(R)-6-fluoro-1-phenyl-2a,3-dihydrocyclobuta[a]naphthalen-4(2H)-one (3aa)}

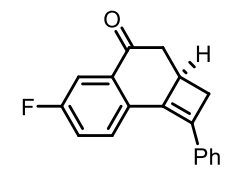

Yellow oil after purification by column chromatography (petroleum ether/ethyl acetate $=50 / 1$ ); $26.1 \mathrm{mg}$, $54 \%$ yield; $[\alpha]_{\mathrm{D}^{20}}=+432.6\left(\mathrm{c}=0.092\right.$, acetone); ${ }^{1} \mathrm{H} \mathrm{NMR}\left(400 \mathrm{MHz}, \mathrm{CDCl}_{3}\right)(\delta, \mathrm{ppm}): 7.84-7.76(\mathrm{~m}, 2 \mathrm{H}), 7.62(\mathrm{~d}, J=7.2 \mathrm{~Hz}, 2 \mathrm{H})$, 7.45-7.41 (m, 2H), 7.38-7.29 (m, 2H), 3.30-3.17 (m, 3H), 2.71-2.65 (m, 2H). ${ }^{13} \mathrm{C}$ NMR (100 MHz, $\left.\mathrm{CDCl}_{3}\right)(\delta, \mathrm{ppm})$ : 
$196.7,163.4,160.9\left({ }^{1} J_{\mathrm{CF}}=248.1 \mathrm{~Hz}\right), 137.1,135.1,134.9,134.5(2), 134.5(6), 133.0(2), 133.0(9)\left({ }^{4} J_{\mathrm{CF}}=3.2 \mathrm{~Hz}\right), 128.6$, 128.5, 127.0, $126.9\left({ }^{3} J_{\mathrm{CF}}=7.2 \mathrm{~Hz}\right), 125.9,120.9,120.7\left({ }^{2} J_{\mathrm{CF}}=22.4 \mathrm{~Hz}\right), 114.6,114.4,45.7,35.7,35.7 . \mathrm{IR}\left(\mathrm{KBr}, v, \mathrm{~cm}^{-}\right.$ $\left.{ }^{1}\right): 3005,2988,1686,1603,1521,1445,826,750$; HRMS (ESI) m/z calcd for $\mathrm{C}_{18} \mathrm{H}_{14} \mathrm{FO}[\mathrm{M}+\mathrm{Na}]^{+} 265.1029$, found 265.1028; HPLC: 97\% ee (Daicel Chiralcel OD-H, hexane/i-PrOH =95/5, flow rate: $0.6 \mathrm{~mL} / \mathrm{min}$, detector: $254 \mathrm{~nm}$, $\mathrm{t}_{\mathrm{R}}($ minor $)=10.487 \mathrm{~min}, \mathrm{t}_{\mathrm{R}}($ major $)=13.519 \min$.

\section{(R)-6-fluoro-1-(p-tolyl)-2a,3-dihydrocyclobuta[a]naphthalen-4(2H)-one (3bb)}

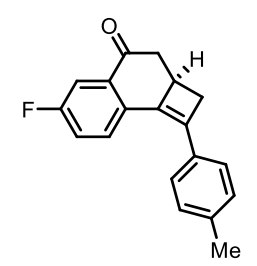

Yellow oil after purification by column chromatography (petroleum ether/ethyl acetate $=50 / 1$ ); $31.2 \mathrm{mg}$, 56\% yield; $[\alpha]_{\mathrm{D}}^{20}=+303.0(\mathrm{c}=0.462$, acetone $) ;{ }^{1} \mathrm{H}$ NMR $\left(400 \mathrm{MHz}, \mathrm{CDCl}_{3}\right)(\delta, \mathrm{ppm}): 7.82-7.70(\mathrm{~m}, 2 \mathrm{H}), 7.49(\mathrm{~d}, J=8.0 \mathrm{~Hz}, 2 \mathrm{H})$, 7.32-7.26 (m, 1H), $7.21(\mathrm{~d}, J=8.0 \mathrm{~Hz}, 2 \mathrm{H}), 3.25-3.14(\mathrm{~m}, 3 \mathrm{H}), 2.70-2.60(\mathrm{~m}, 2 \mathrm{H}), 2.39(\mathrm{~s}, 3 \mathrm{H}) .{ }^{13} \mathrm{C}$ NMR $(100 \mathrm{MHz}$, $\left.\mathrm{CDCl}_{3}\right)(\delta, \mathrm{ppm}): 196.8,160.0,137.9,135.2\left({ }^{1} J_{\mathrm{CF}}=269.9 \mathrm{~Hz}\right), 133.6,133.5,133.4,132.3,128.1,127.8,127.4,126.2$, 114.1, 55.4, 45.8, 35.8, 35.4. IR (KBr, $\left.v, \mathrm{~cm}^{-1}\right): 3025,2914,1686,1603,1511,1417,813$, 749; HRMS (ESI) m/z calcd for $\mathrm{C}_{19} \mathrm{H}_{16} \mathrm{FO}[\mathrm{M}+\mathrm{H}]^{+}$279.1185, found 279.1187; HPLC: $>99 \%$ ee (Daicel Chiralpak IA, hexane/i-PrOH =95/5, flow rate: $0.6 \mathrm{~mL} / \mathrm{min}$, detector: $254 \mathrm{~nm}, \mathrm{t}_{\mathrm{R}}($ minor $)=8.694 \mathrm{~min}, \mathrm{t}_{\mathrm{R}}($ major $)=9.912 \mathrm{~min}$.

(R)-6-chloro-1-phenyl-2a,3-dihydrocyclobuta[a]naphthalen-4(2H)-one (3cc)

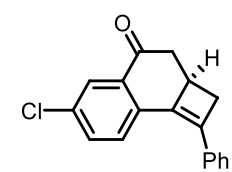

Yellow oil after purification by column chromatography (petroleum ether/ethyl acetate $=50 / 1$ ); $35.4 \mathrm{mg}$, $63 \%$ yield; $[\alpha]_{\mathrm{D}}^{20}=+127.0(\mathrm{c}=0.126$, acetone $) ;{ }^{1} \mathrm{H}$ NMR $\left(400 \mathrm{MHz}, \mathrm{CDCl}_{3}\right)(\delta, \mathrm{ppm}): 8.07(\mathrm{~d}, J=2.4 \mathrm{~Hz}, 1 \mathrm{H}), 7.77(\mathrm{~d}, J=8.4 \mathrm{~Hz}$, $1 \mathrm{H}), 7.61(\mathrm{~d}, J=7.2 \mathrm{~Hz}, 2 \mathrm{H}), 7.57-7.55(\mathrm{~m}, 1 \mathrm{H}), 7.46-7.33(\mathrm{~m}, 3 \mathrm{H}), 3.31-3.15(\mathrm{~m}, 3 \mathrm{H}), 2.74-2.62(\mathrm{~m}, 2 \mathrm{H}) .{ }^{13} \mathrm{C}$ NMR $\left(100 \mathrm{MHz}, \mathrm{CDCl}_{3}\right)(\delta, \mathrm{ppm}): 196.6,138.2,134.9,134.9,134.8,134.1,133.6,133.4,128.7,128.1,126.3,126.0,45.7,35.9$, 35.6. IR $\left(\mathrm{KBr}, v, \mathrm{~cm}^{-1}\right): 3051,2983,1685,1585,1492,1462,825,742$; HRMS (ESI) $\mathrm{m} / \mathrm{z}$ calcd for $\mathrm{C}_{18} \mathrm{H}_{14} \mathrm{ClO}[\mathrm{M}+\mathrm{H}]^{+}$ 281.0733, found 281.0734; HPLC: 96\% ee (Daicel Chiralcel OD-H, hexane/i-PrOH $=95 / 5$, flow rate: $0.6 \mathrm{~mL} / \mathrm{min}$, detector: $254 \mathrm{~nm}, \mathrm{t}_{\mathrm{R}}($ minor $)=9.772 \mathrm{~min}, \mathrm{t}_{\mathrm{R}}($ major $)=12.988 \mathrm{~min}$.

\section{(R)-6-chloro-1-(4-methoxyphenyl)-2a,3-dihydrocyclobuta[a]naphthalen-4(2H)-one (3dd)}

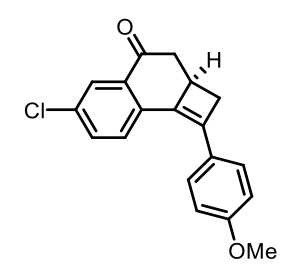

Yellow oil after purification by column chromatography (petroleum ether/ethyl acetate $=50 / 1$ ); $37.8 \mathrm{mg}, 61 \%$ yield; $[\alpha]_{\mathrm{D}}{ }^{20}=+312.0(\mathrm{c}=0.150$, acetone $) ;{ }^{1} \mathrm{H}$ NMR $\left(400 \mathrm{MHz}, \mathrm{CDCl}_{3}\right)(\delta, \mathrm{ppm}): 8.03(\mathrm{~d}, J=2.4 \mathrm{~Hz}, 1 \mathrm{H}), 7.71(\mathrm{~d}, J=8.4 \mathrm{~Hz}$, 1H), 7.59-7.47 (m, 3H), 6.96-6.90 (m, 2H), $3.85(\mathrm{~s}, 3 \mathrm{H}), 3.26-3.12(\mathrm{~m}, 3 \mathrm{H}), 2.70-2.59(\mathrm{~m}, 2 \mathrm{H}) .{ }^{13} \mathrm{C}$ NMR $(100 \mathrm{MHz}$, $\left.\mathrm{CDCl}_{3}\right)(\delta, \mathrm{ppm}): 196.8,160.0,137.9,135.2,133.6,133.5,133.4,132.3,128.1,127.8,127.4,126.2,114.1,55.4,45.8$, 35.8, 35.4. IR (KBr, $\left.v, \mathrm{~cm}^{-1}\right): 3004,2932,1685,1602,1510,1420,826,748$; HRMS (ESI) m/z calcd for $\mathrm{C}_{19} \mathrm{H}_{15} \mathrm{ClNaO}_{2}$ 
$[\mathrm{M}+\mathrm{Na}]^{+}$333.0658, found 333.0657; HPLC: 99\% ee (Daicel Chiralpak IA, hexane/i-PrOH =95/5, flow rate: $0.6 \mathrm{~mL} / \mathrm{min}$, detector: $254 \mathrm{~nm}, \mathrm{t}_{\mathrm{R}}($ minor $)=12.863 \mathrm{~min}, \mathrm{t}_{\mathrm{R}}($ major $)=17.249 \mathrm{~min}$. 
Chiral HPLC analyses of products 3

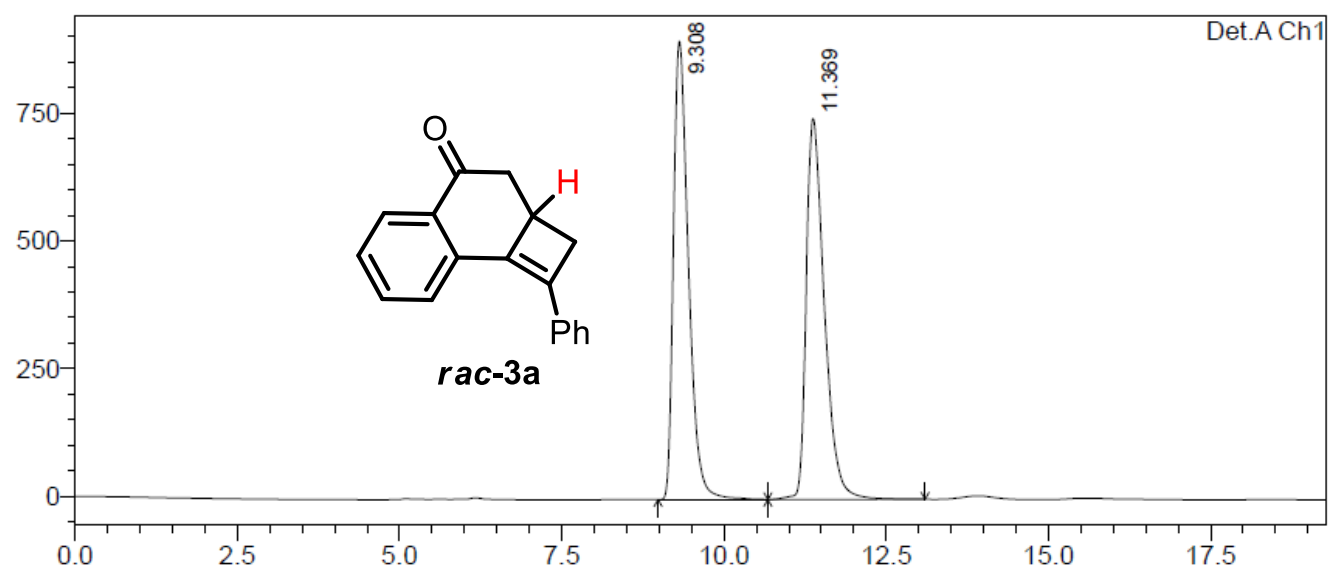

\begin{tabular}{|r|r|r|r|r|r|}
\multicolumn{1}{|c|}{ Peak\# } & Ret. Time & Area & Height & \multicolumn{1}{c|}{ Area \% } & Height \% \\
\hline 1 & 9.308 & 14433520 & 896964 & 49.870 & 54.625 \\
\hline 2 & 11.369 & 14508891 & 745090 & 50.130 & 45.375 \\
\hline Total & & 28942411 & 1642054 & 100.000 & 100.000 \\
\hline
\end{tabular}

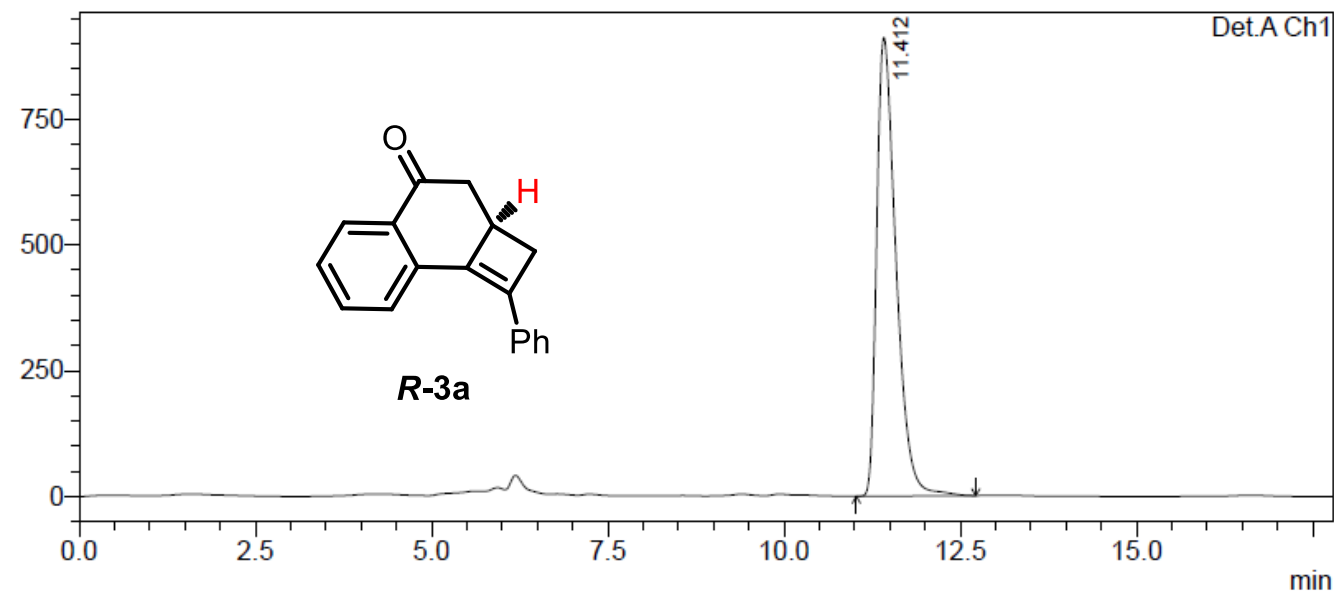

\begin{tabular}{|r|r|r|r|r|r|}
\hline \multicolumn{1}{|c|}{ Peak\# } & Ret. Time & Area & Height & \multicolumn{1}{c|}{ Area \% } & \multicolumn{1}{c|}{ Height \% } \\
\hline 1 & 11.412 & 17417895 & 912048 & 100.000 & 100.000 \\
\hline Total & & 17417895 & 912048 & 100.000 & 100.000 \\
\hline
\end{tabular}

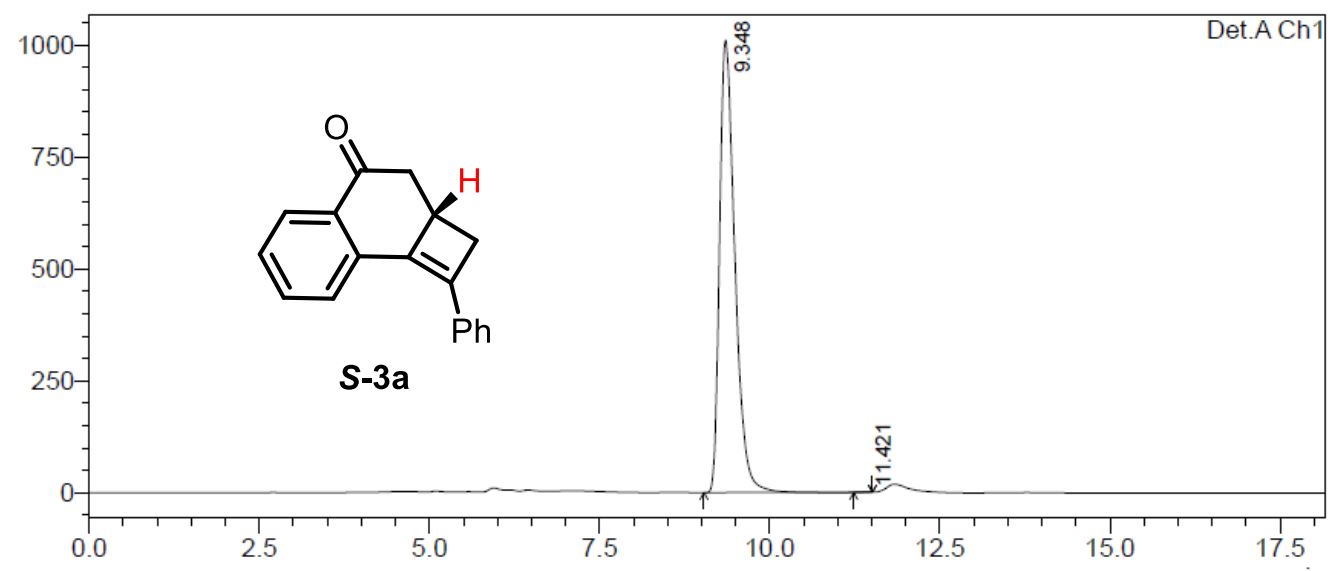

\begin{tabular}{|r|r|r|r|r|r|}
\hline \multicolumn{1}{|c|}{ Peak\# } & Ret. Time & \multicolumn{1}{c|}{ Area } & Height & \multicolumn{1}{c|}{ Area \% } & Height \% \\
\hline 1 & 9.348 & 15787832 & 1011050 & 99.977 & 99.964 \\
\hline 2 & 11.421 & 3706 & 368 & 0.023 & 0.036 \\
\hline Total & & 15791538 & 1011418 & 100.000 & 100.000 \\
\hline
\end{tabular}




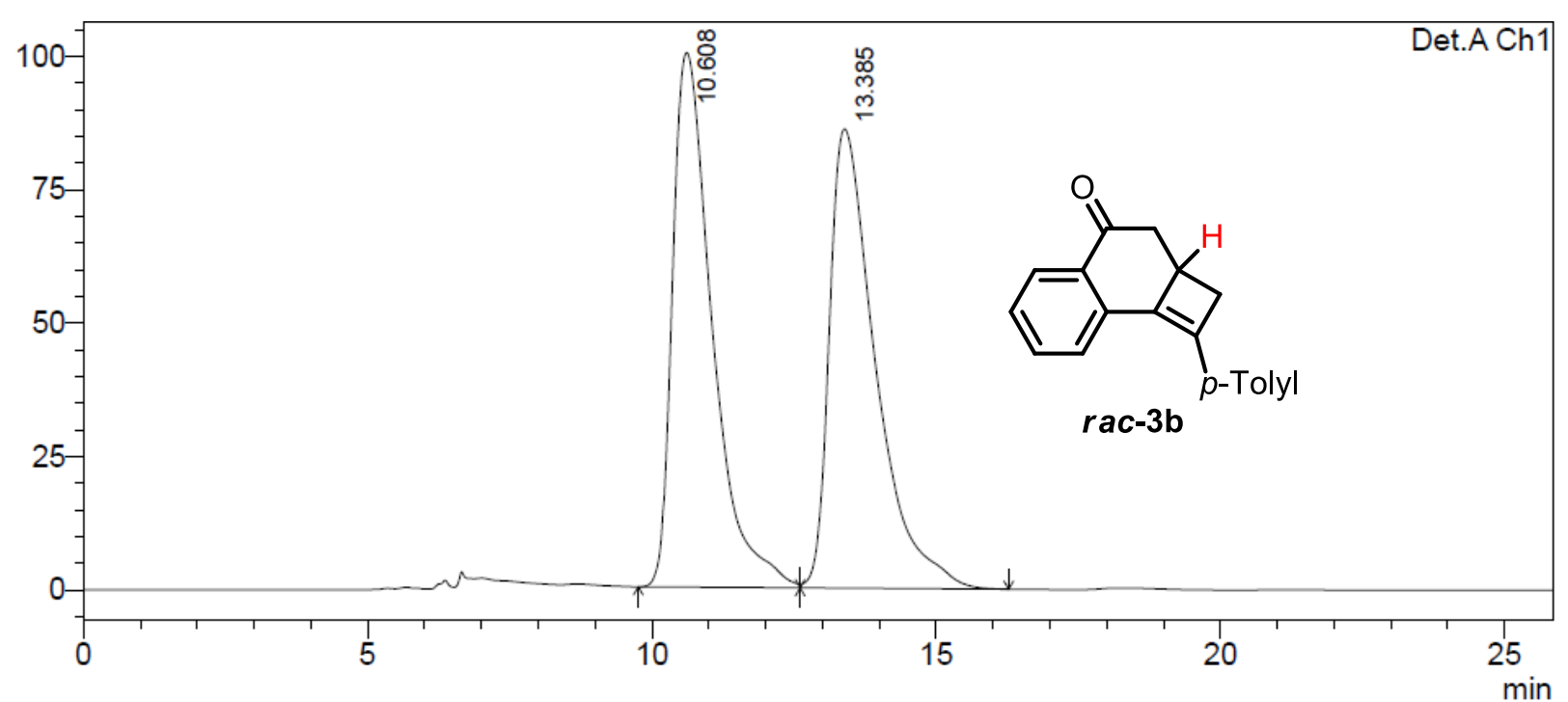

\begin{tabular}{|r|r|r|r|r|r|}
\hline \multicolumn{1}{|c|}{ Peak\# } & Ret. Time & \multicolumn{1}{c|}{ Area } & Height & \multicolumn{1}{c|}{ Area \% } & \multicolumn{1}{c|}{ Height \% } \\
\hline 1 & 10.608 & 4855996 & 100131 & 50.041 & 53.794 \\
\hline 2 & 13.385 & 4848102 & 86005 & 49.959 & 46.206 \\
\hline Total & & 9704098 & 186136 & 100.000 & 100.000 \\
\hline
\end{tabular}

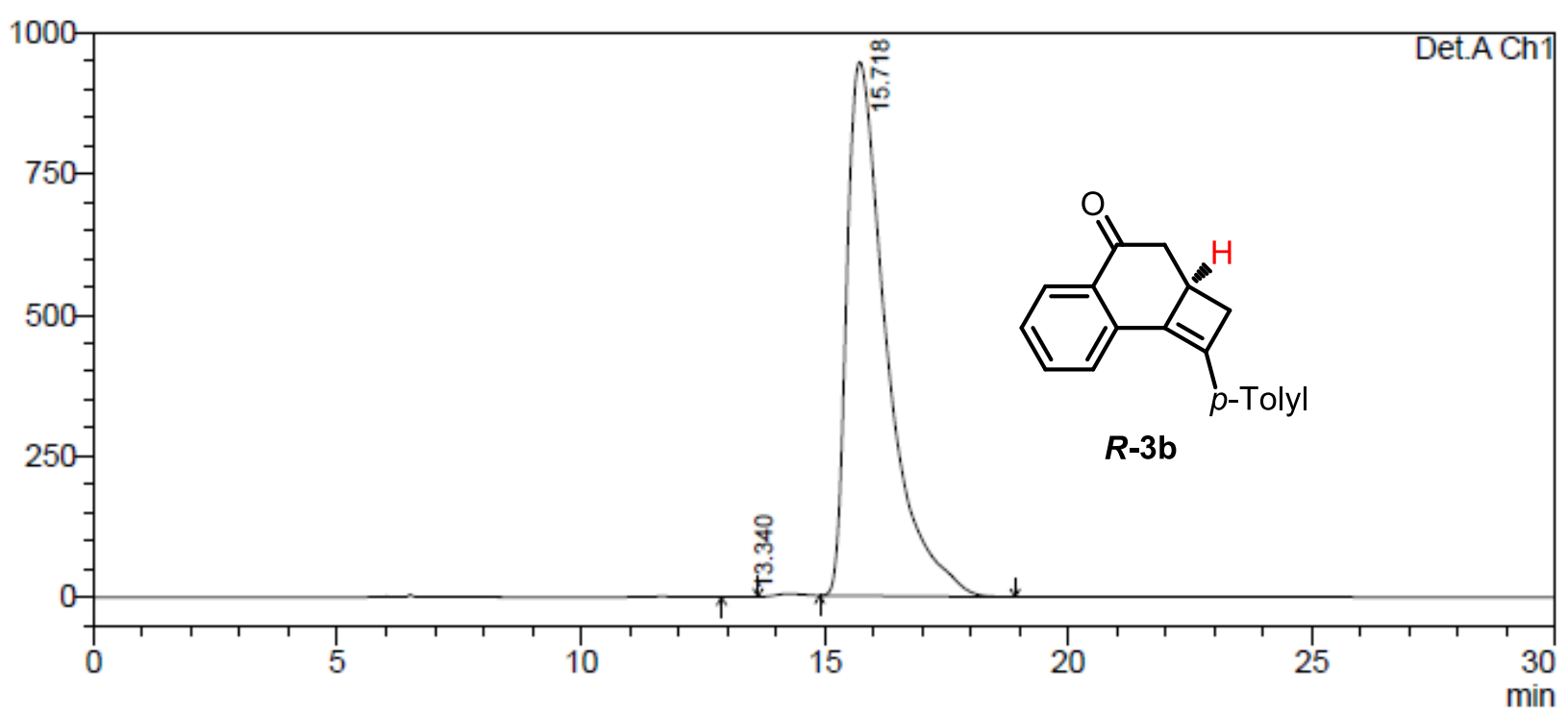

\begin{tabular}{|r|r|r|r|r|r|}
\hline \multicolumn{1}{|c|}{ Peak\# } & Ret. Time & \multicolumn{1}{c|}{ Area } & \multicolumn{1}{c|}{ Height } & Area \% & \multicolumn{1}{c|}{ Height \% } \\
\hline 1 & 13.340 & 10841 & 434 & 0.020 & 0.046 \\
\hline 2 & 15.718 & 53805793 & 945111 & 99.980 & 99.954 \\
\hline Total & & 53816634 & 945545 & 100.000 & 100.000 \\
\hline
\end{tabular}



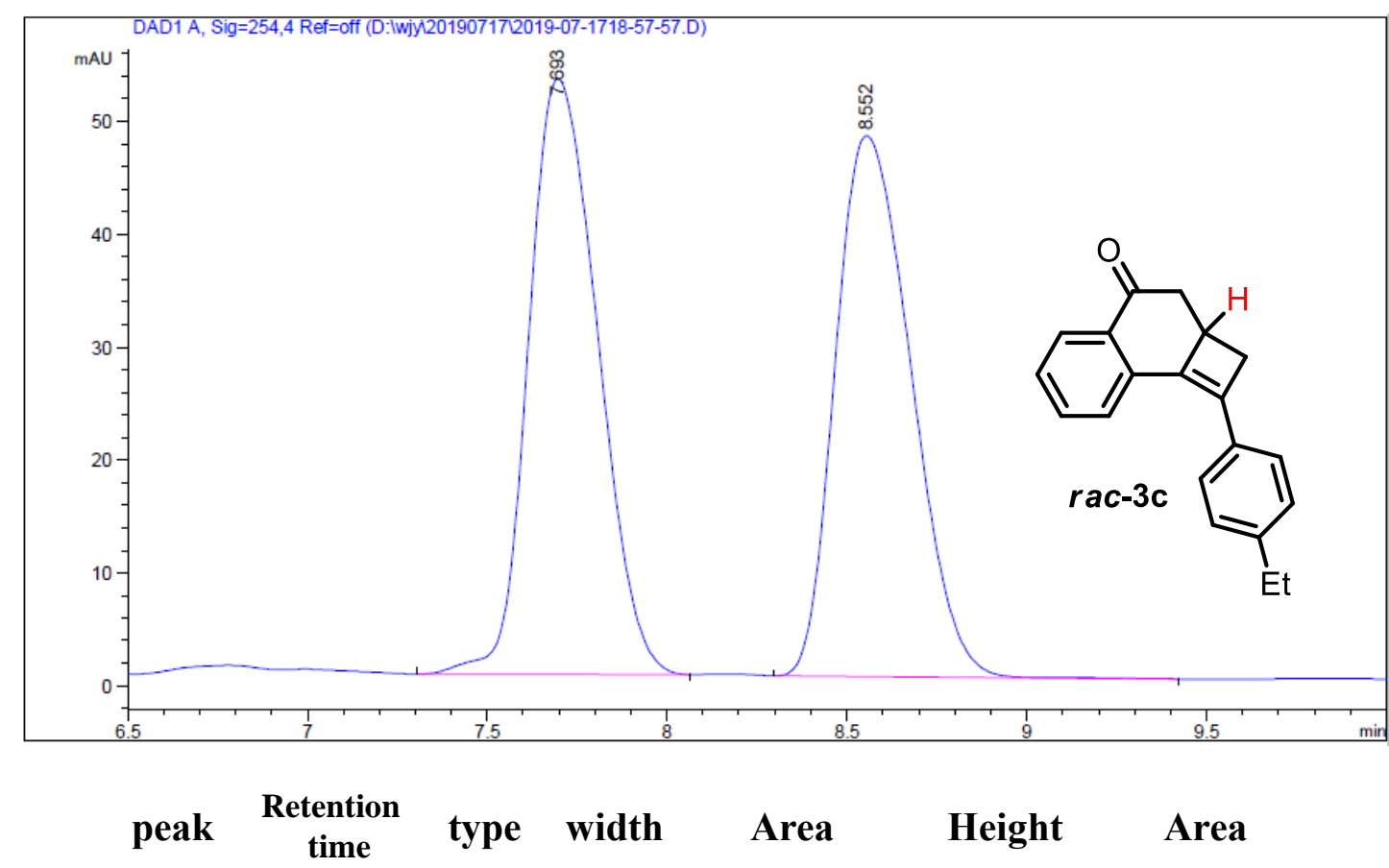

峰 保留时间 类型 峰宽峰面积峰高峰面积

$\begin{array}{cccccc}\# & \text { [min] } & \text { [min] } & \text { [mAU*s] } & \text { [mAU] } & \% \\ -1 & 7.693 \mathrm{BB} & 0.2138 & 704.92413 & 52.77470 & 50.1814 \\ 2 & 8.552 \mathrm{BB} & 0.2343 & 699.82721 & 47.93524 & 49.8186\end{array}$
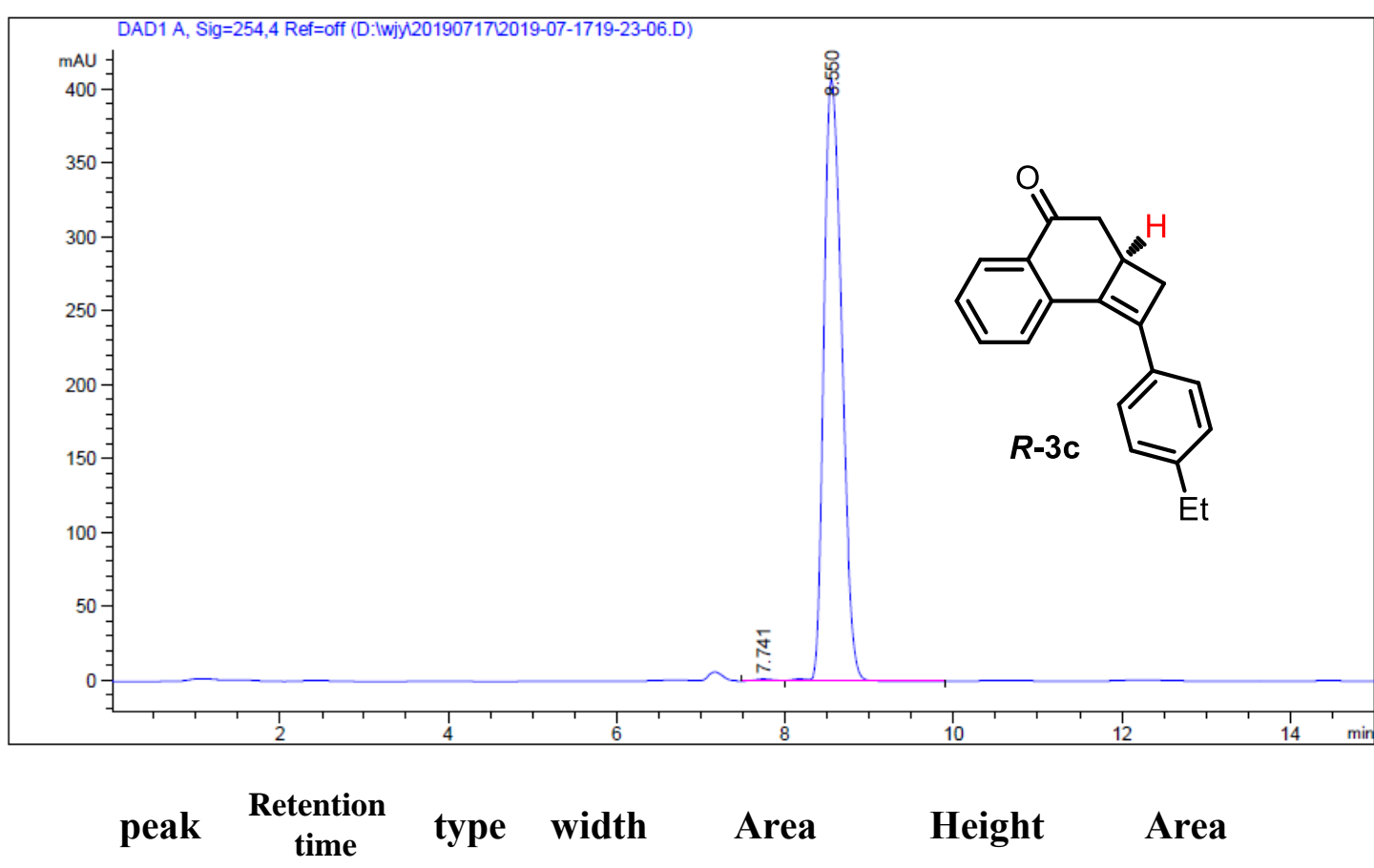

峰保留时间类型峰宽峰面积峰高峰面积

\# [min] [min] [mAU*s $] \quad[\mathrm{mAU}] \quad \%$

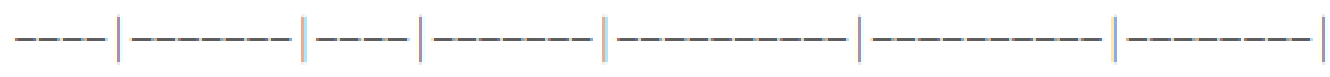
17.741 BV E
0. 2249
19. 77050
1. 30383
0. 3287

28.550 VB R

0.23735995 .68799

407. 24240

99.6713 


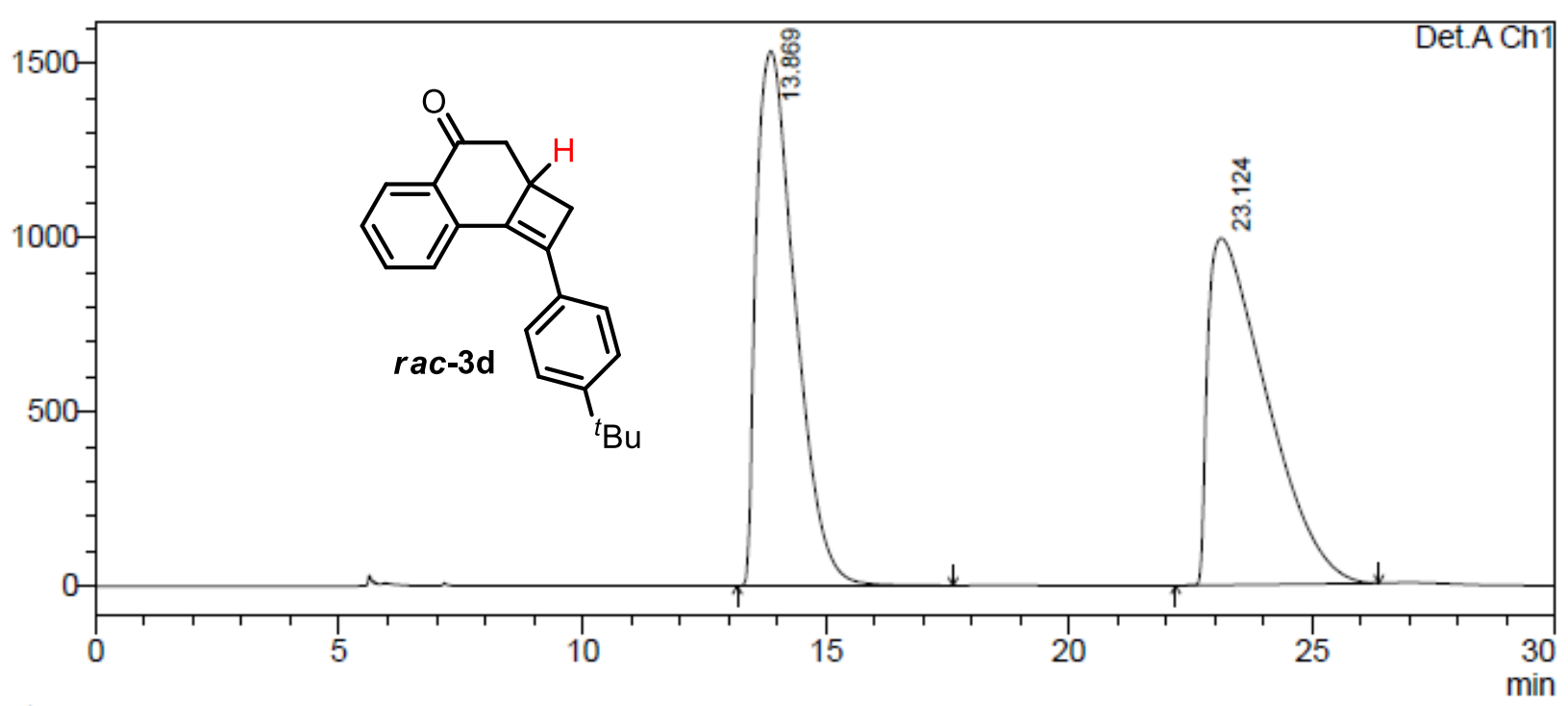

\begin{tabular}{|r|r|r|r|r|r|}
\hline \multicolumn{1}{|c|}{ Peak\# } & Ret. Time & \multicolumn{1}{c|}{ Area } & \multicolumn{1}{c|}{ Height } & \multicolumn{1}{|c|}{ Area \% } & \multicolumn{1}{|c|}{ Height \% } \\
\hline 1 & 13.869 & 84957991 & 1533317 & 50.303 & 60.655 \\
\hline 2 & 23.124 & 83933736 & 994595 & 49.697 & 39.345 \\
\hline Total & & 168891727 & 2527912 & 100.000 & 100.000 \\
\hline
\end{tabular}

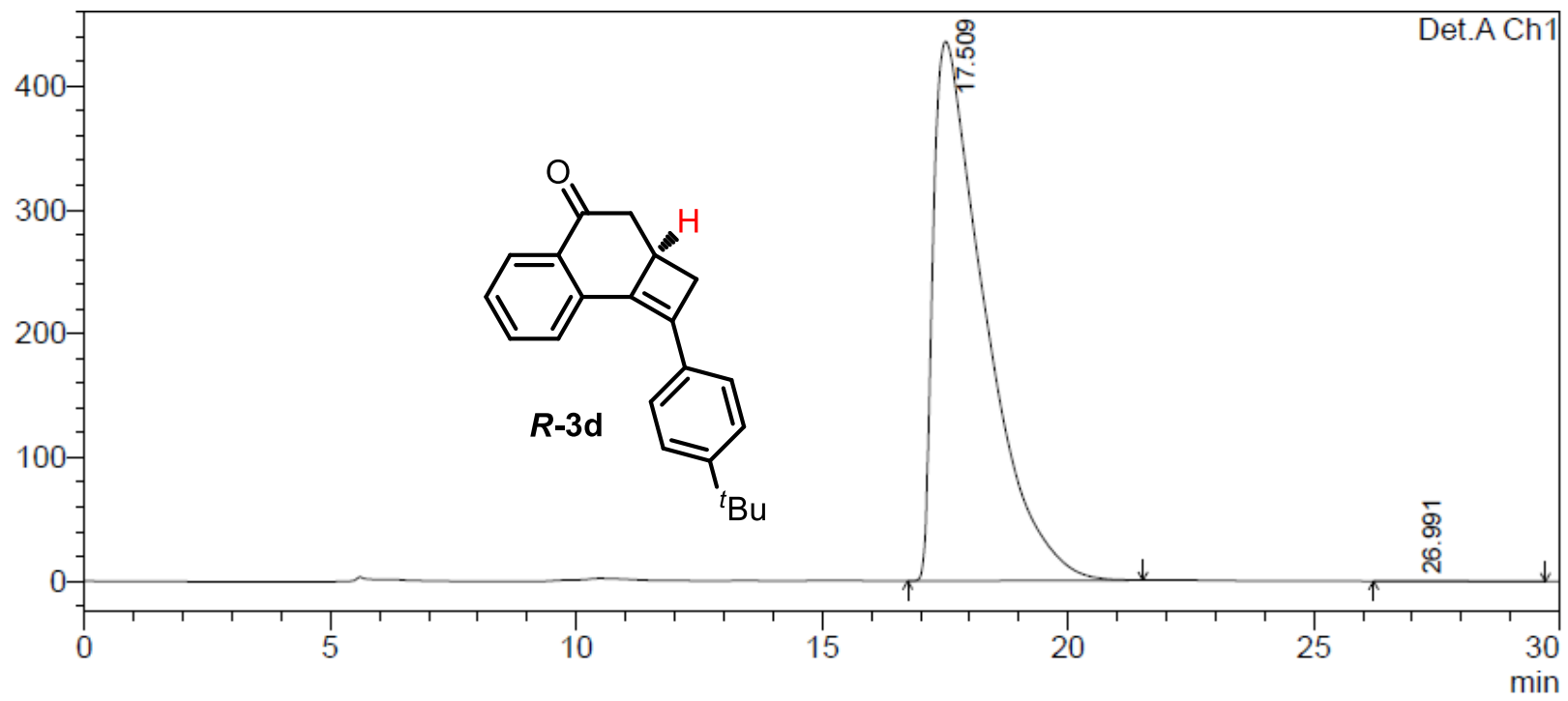

\begin{tabular}{|r|r|r|r|r|r|}
\hline \multicolumn{1}{|c|}{ Peak\# } & Ret. Time & \multicolumn{1}{c|}{ Area } & \multicolumn{1}{c|}{ Height } & \multicolumn{1}{c|}{ Area \% } & \multicolumn{1}{c|}{ Height \% } \\
\hline 1 & 17.509 & 31897246 & 435898 & 99.894 & 99.919 \\
\hline 2 & 26.991 & 33854 & 352 & 0.106 & 0.081 \\
\hline Total & & 31931099 & 436250 & 100.000 & 100.000 \\
\hline
\end{tabular}




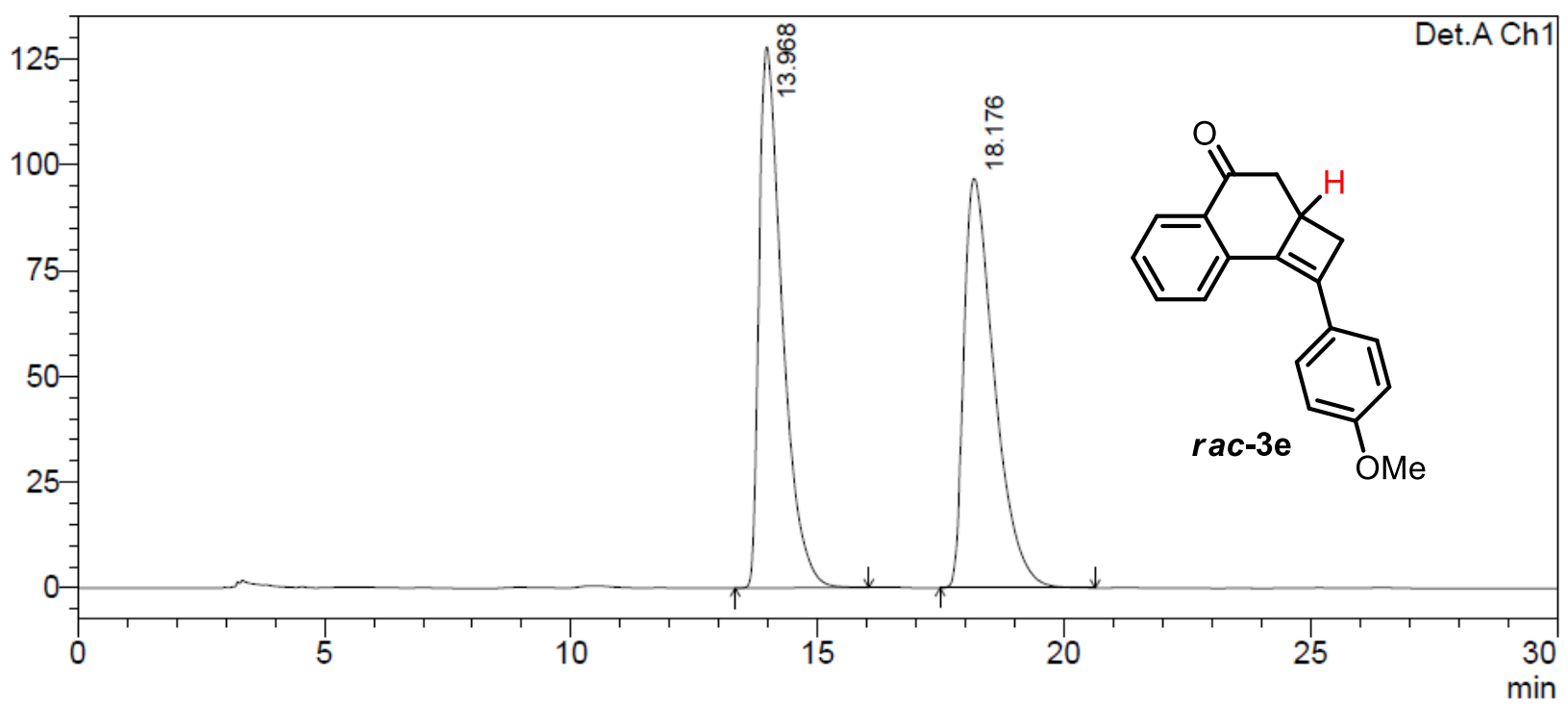

\begin{tabular}{|r|r|r|r|r|r|}
\hline \multicolumn{1}{|c|}{ Peak\# } & Ret. Time & Area & Height & \multicolumn{1}{|c|}{ Area \% } & \multicolumn{1}{|c|}{ Height \% } \\
\hline 1 & 13.968 & 4209276 & 127911 & 50.243 & 56.911 \\
\hline 2 & 18.176 & 4168586 & 96845 & 49.757 & 43.089 \\
\hline Total & & 8377862 & 224756 & 100.000 & 100.000 \\
\hline
\end{tabular}

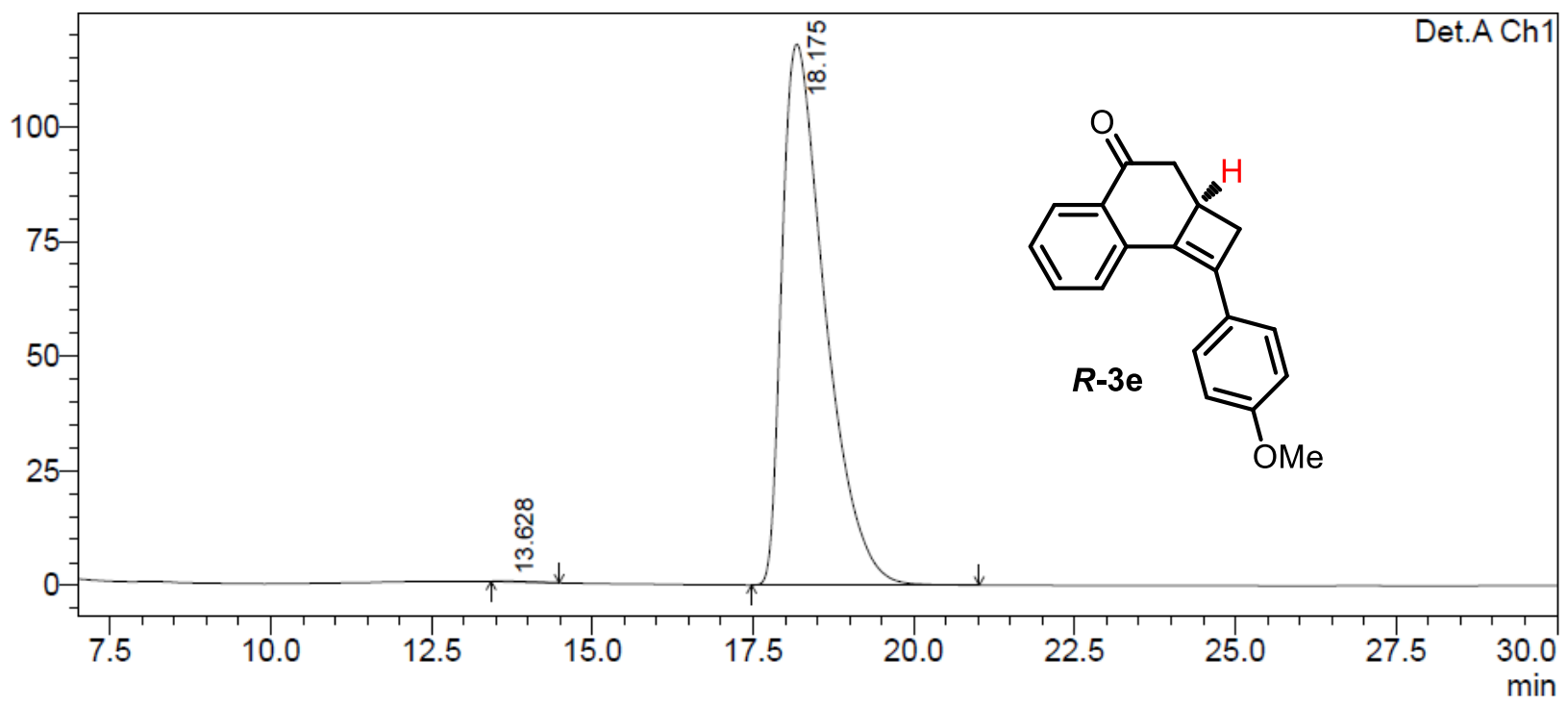

\begin{tabular}{|r|r|r|r|r|r|}
\hline \multicolumn{1}{|c|}{ Peak\# } & Ret. Time & \multicolumn{1}{c|}{ Area } & \multicolumn{1}{c|}{ Height } & \multicolumn{1}{c|}{ Area \% } & \multicolumn{1}{c|}{ Height \% } \\
\hline 1 & 13.628 & 3131 & 82 & 0.057 & 0.070 \\
\hline 2 & 18.175 & 5520499 & 117953 & 99.943 & 99.930 \\
\hline Total & & 5523631 & 118035 & 100.000 & 100.000 \\
\hline
\end{tabular}



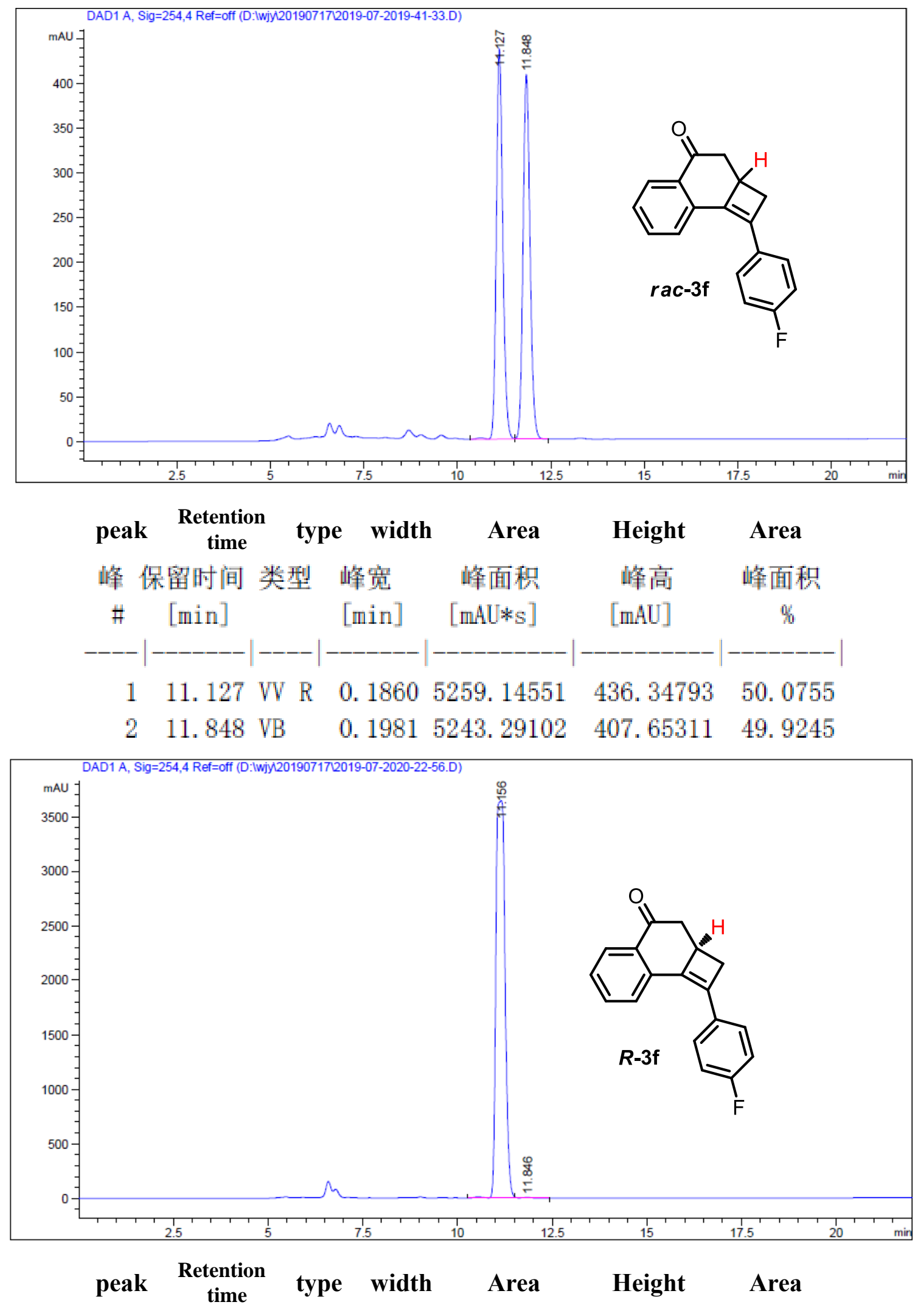

峰 保留时间 类型 峰宽峰面积峰高峰面积

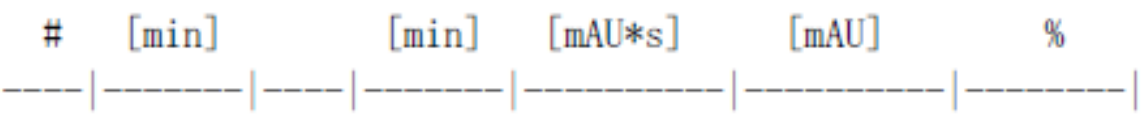

$1 \quad 11.156$ VV R $\quad 0.2331 \quad 6.11520 \mathrm{e} 4 \quad 3644.91211 \quad 99.8084$

$2 \quad 11.846$ VB E $\quad 0.2433 \quad 117.42107 \quad 7.16215 \quad 0.1916$ 

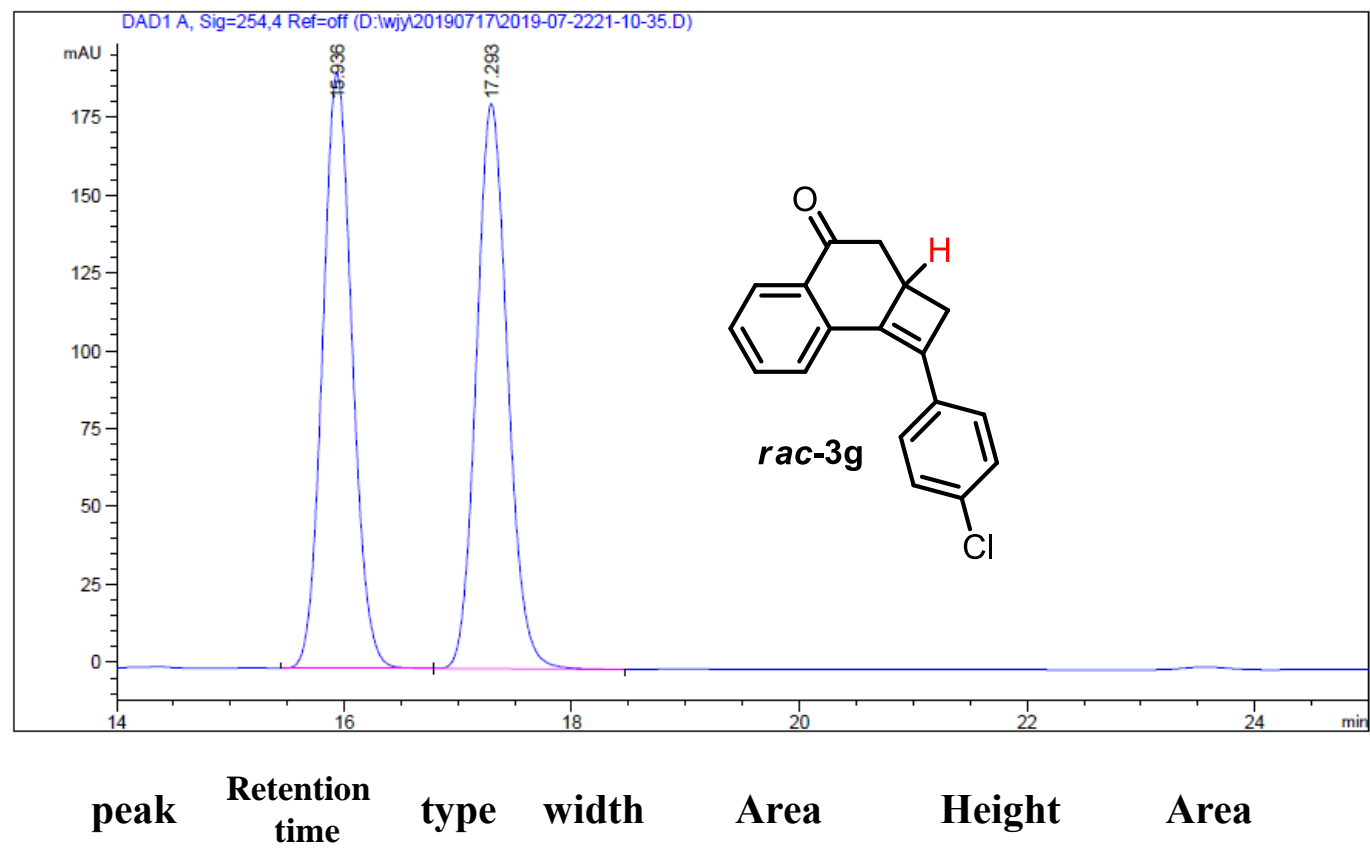

峰 保留时间 类型 峰宽峰面积峰高峰面积

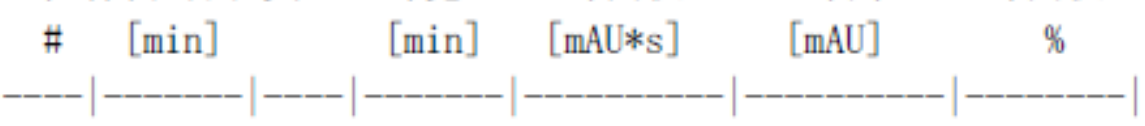
$1 \quad 15.936 \mathrm{BB}$
0. $27863451.18750 \quad 191.17418 \quad 49.9131$

$217.293 \mathrm{BB}$

$\begin{array}{llll}0.2968 & 3463.20117 & 181.37451 & 50.0869\end{array}$

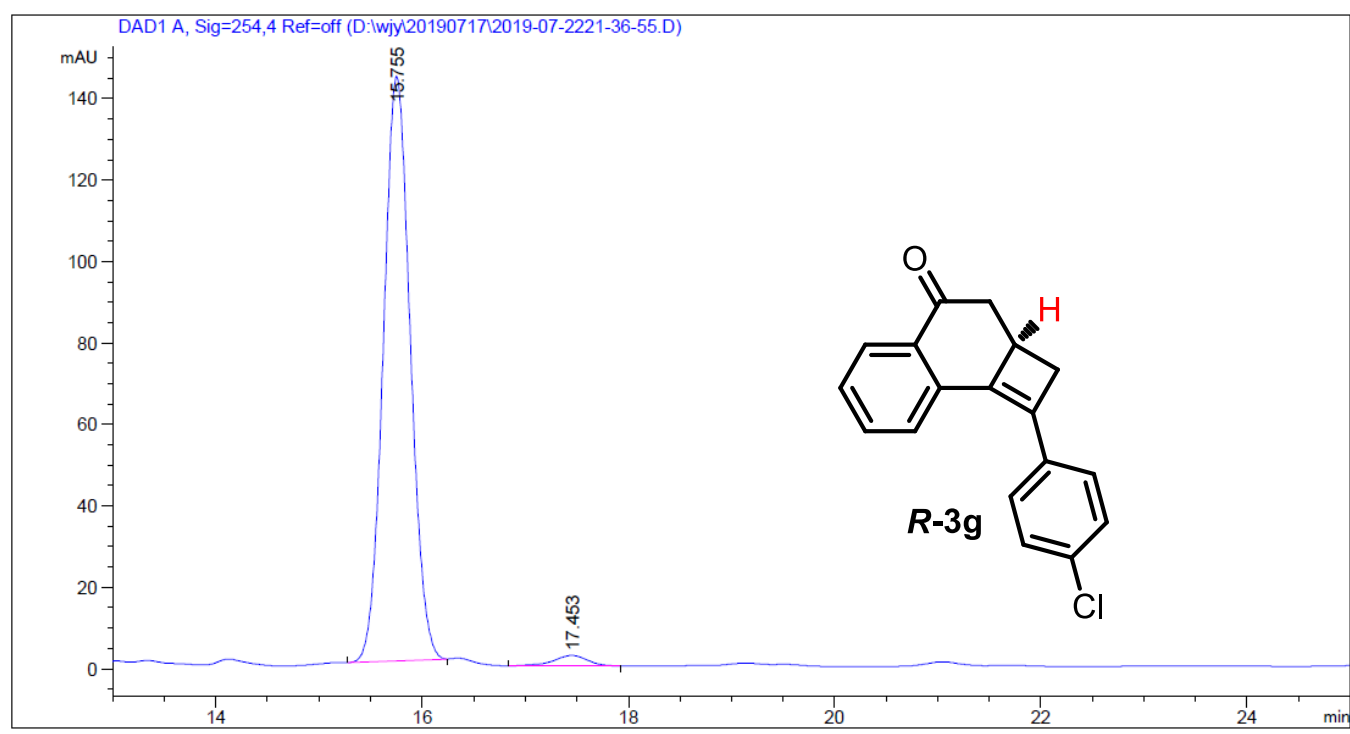

peak $\begin{gathered}\text { Retention } \\ \text { time }\end{gathered}$ type width Area Height Area

\begin{tabular}{|c|c|c|c|c|c|c|}
\hline $\begin{array}{r}\text { 峰 } \\
\#\end{array}$ & $\begin{array}{c}\text { 保留时间 } \\
\text { [min] }\end{array}$ & 类型 & $\begin{array}{l}\text { 峰宽 } \\
\text { [min] }\end{array}$ & $\begin{array}{c}\text { 峰面积 } \\
\text { [mAU*s] }\end{array}$ & $\begin{array}{r}\text { 峰高 } \\
\text { [mAU] }\end{array}$ & $\begin{array}{c}\text { 峰面积 } \\
\%\end{array}$ \\
\hline 1 & 15. 755 & BB & 0.2812 & 2599. 72290 & 143.63481 & 97.8137 \\
\hline 2 & 17. 453 & $\mathrm{DP}$ & 0.3435 & 58.10947 & 2. 57324 & 2. 1863 \\
\hline
\end{tabular}




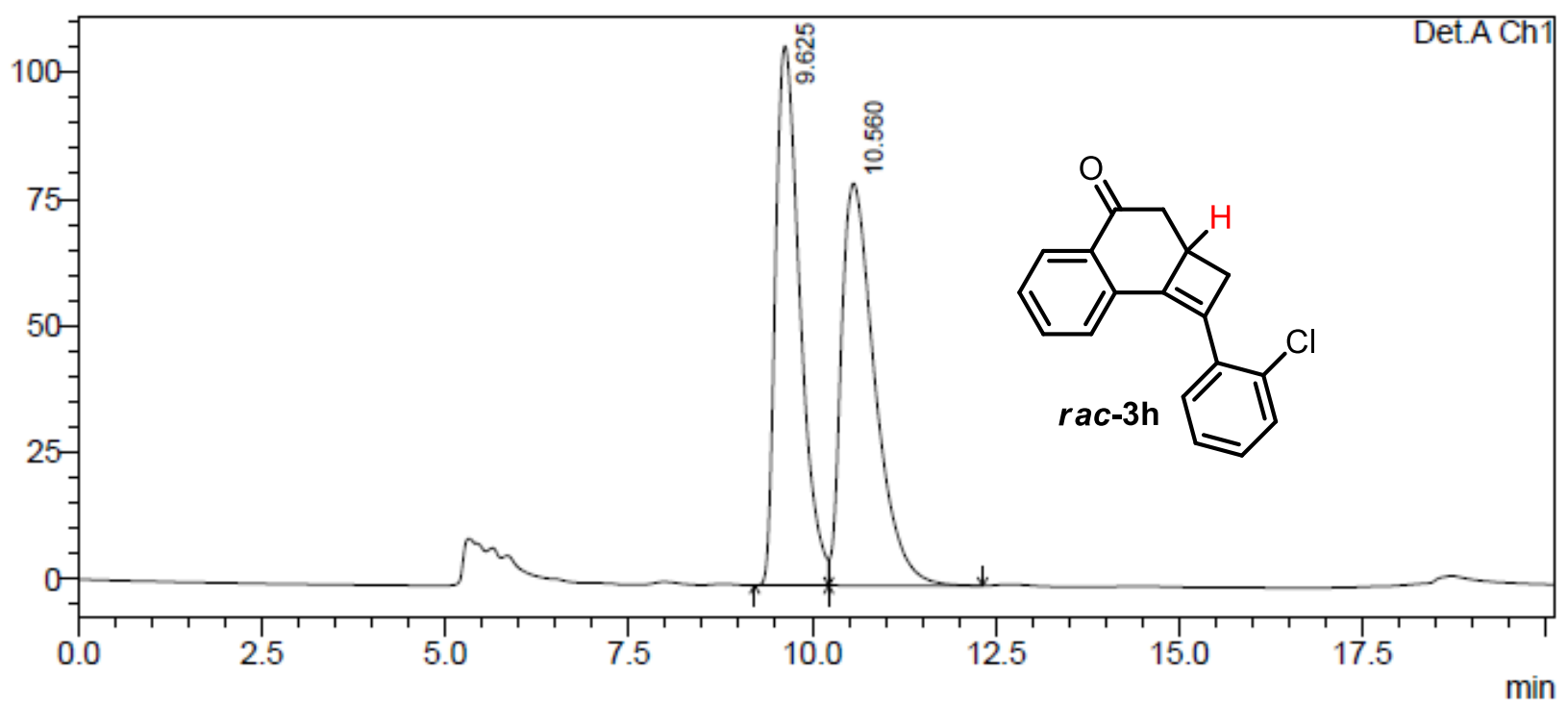

\begin{tabular}{|r|r|r|r|r|r|}
\hline \multicolumn{1}{|c|}{ Peak\# } & \multicolumn{1}{|c|}{ Ret. Time } & \multicolumn{1}{c|}{ Area } & \multicolumn{1}{c|}{ Height } & Area \% & \multicolumn{1}{c|}{ Height \% } \\
\hline 1 & 9.625 & 2475093 & 106377 & 49.458 & 57.283 \\
\hline 2 & 10.560 & 2529302 & 79327 & 50.542 & 42.717 \\
\hline Total & & 5004395 & 185704 & 100.000 & 100.000 \\
\hline
\end{tabular}

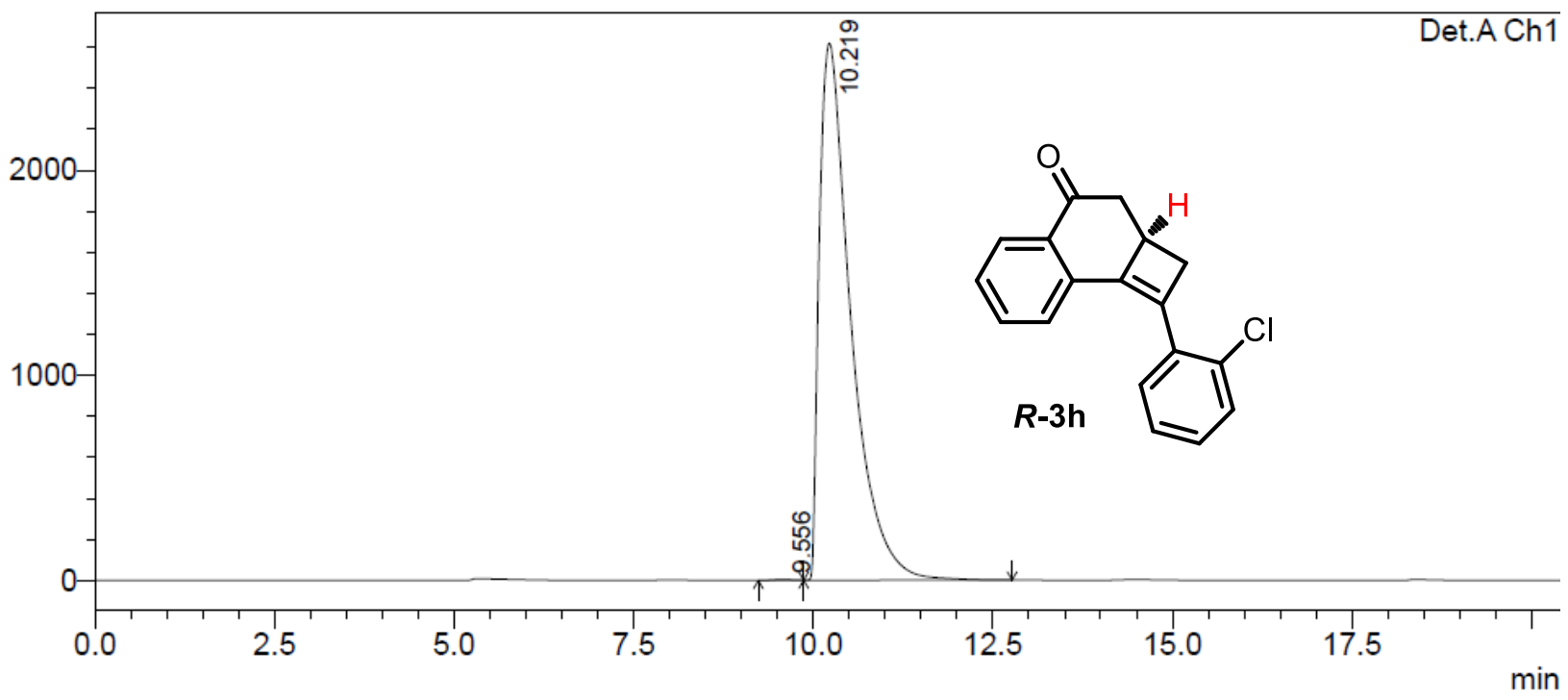

\begin{tabular}{|r|r|r|r|r|r|}
\hline \multicolumn{1}{|c|}{ Peak\# } & \multicolumn{1}{|c|}{ Ret. Time } & \multicolumn{1}{c|}{ Area } & Height & \multicolumn{1}{|c|}{ Area \% } & \multicolumn{1}{c|}{ Height \% } \\
\hline 1 & 9.556 & 81661 & 3891 & 0.102 & 0.148 \\
\hline 2 & 10.219 & 80241447 & 2618692 & 99.898 & 99.852 \\
\hline Total & & 80323108 & 2622583 & 100.000 & 100.000 \\
\hline
\end{tabular}




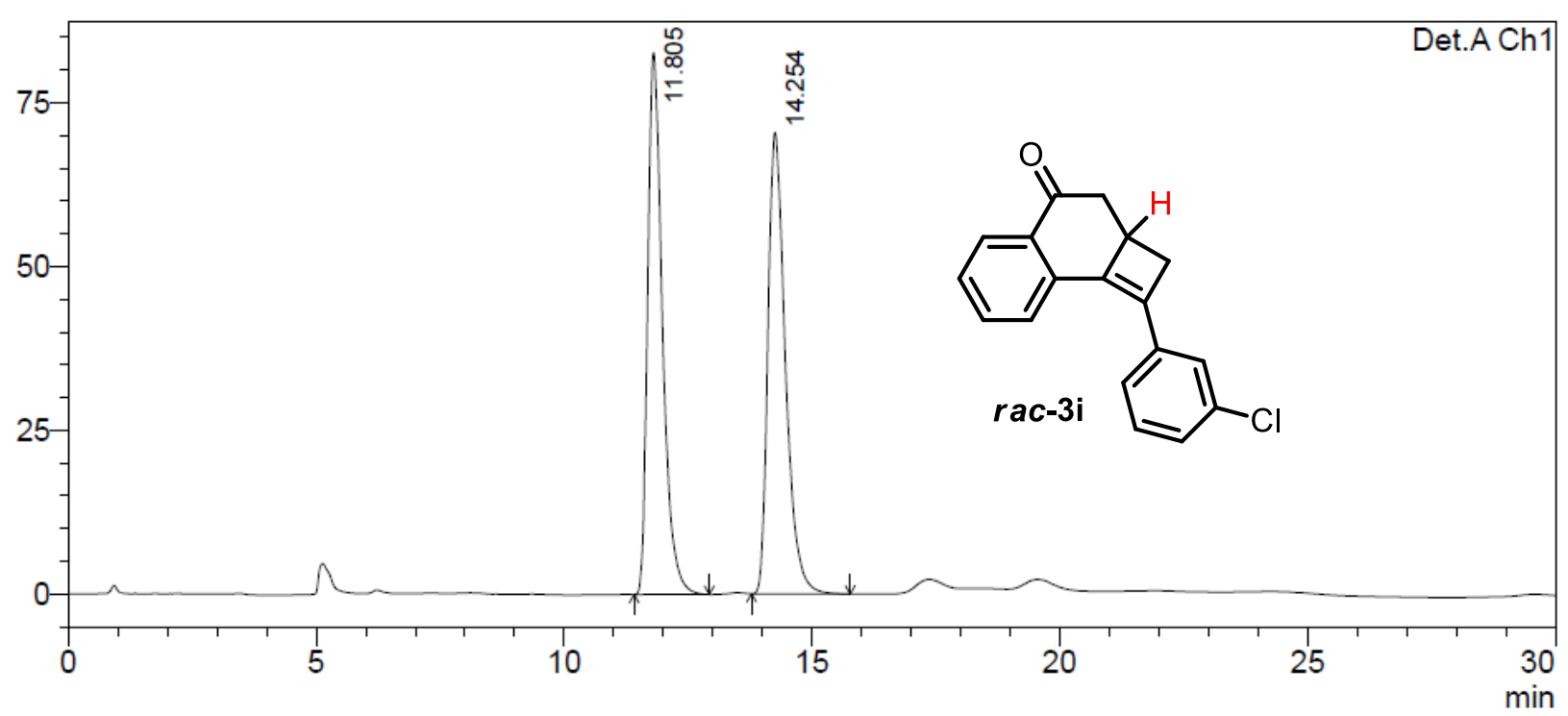

\begin{tabular}{|r|r|r|r|r|r|}
\hline \multicolumn{1}{|c|}{ Peak\# } & Ret. Time & \multicolumn{1}{c|}{ Area } & Height & \multicolumn{1}{c|}{ Area \% } & \multicolumn{1}{c|}{ Height \% } \\
\hline 1 & 11.805 & 1717561 & 82787 & 49.966 & 54.017 \\
\hline 2 & 14.254 & 1719890 & 70474 & 50.034 & 45.983 \\
\hline Total & & 3437451 & 153261 & 100.000 & 100.000 \\
\hline
\end{tabular}

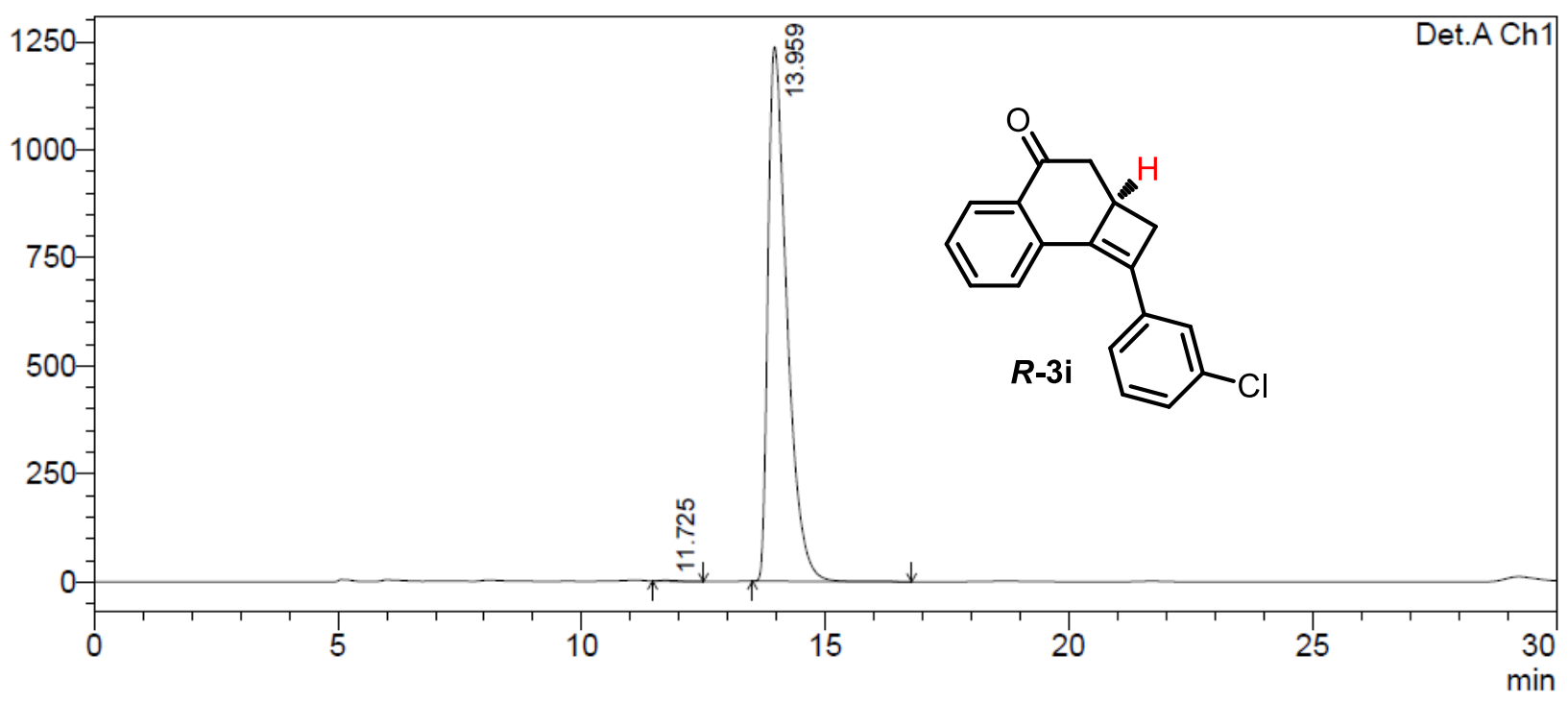

\begin{tabular}{|r|r|r|r|r|r|}
\hline \multicolumn{1}{|c|}{ Peak\# } & Ret. Time & \multicolumn{1}{c|}{ Area } & \multicolumn{1}{c|}{ Height } & \multicolumn{1}{c|}{ Area \% } & \multicolumn{1}{c|}{ Height \% } \\
\hline 1 & 11.725 & 45699 & 1899 & 0.137 & 0.153 \\
\hline 2 & 13.959 & 33429408 & 1237069 & 99.863 & 99.847 \\
\hline Total & & 33475106 & 1238968 & 100.000 & 100.000 \\
\hline
\end{tabular}




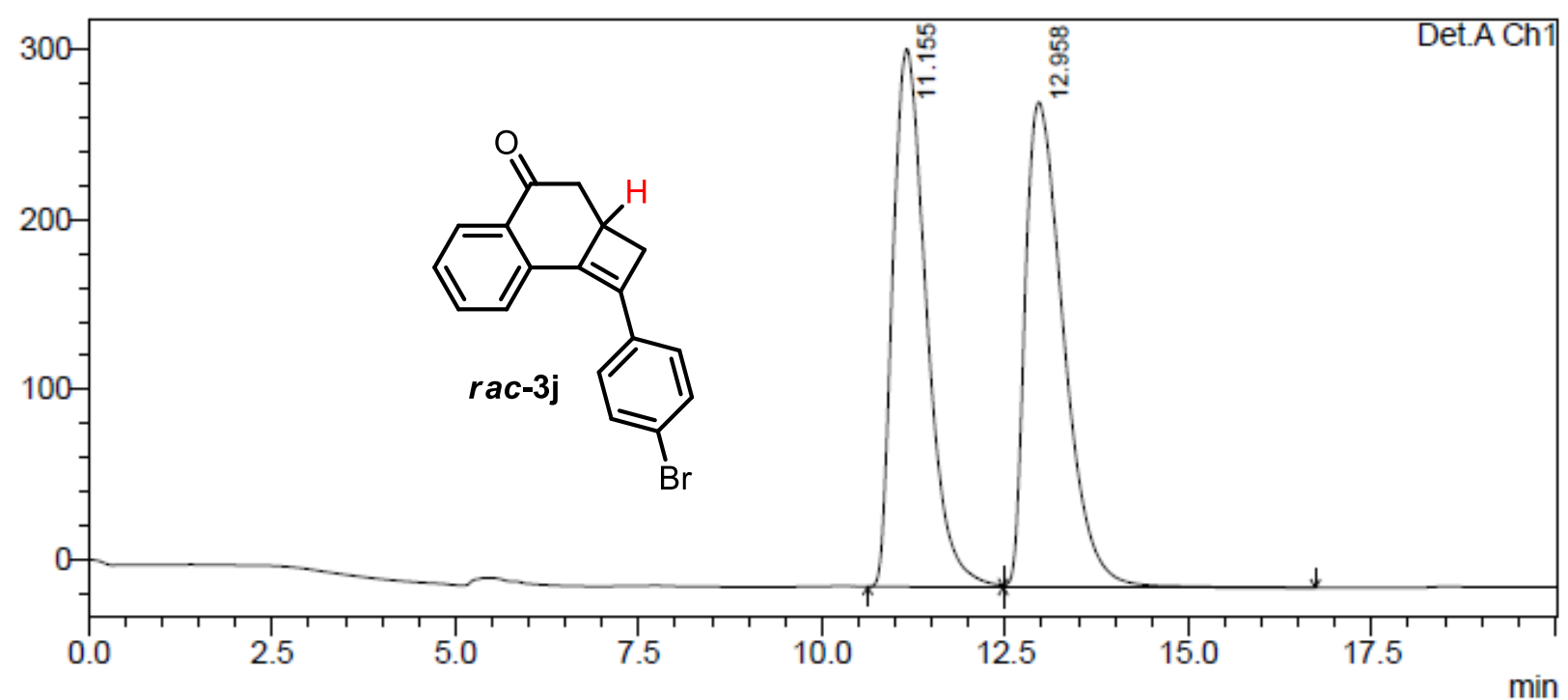

\begin{tabular}{|r|r|r|r|r|r|}
\hline \multicolumn{1}{|c|}{ Peak\# } & Ret. Time & Area & Height & \multicolumn{1}{|c|}{ Area \% } & Height \% \\
\hline 1 & 11.155 & 10089420 & 316763 & 49.213 & 52.609 \\
\hline 2 & 12.958 & 10412154 & 285345 & 50.787 & 47.391 \\
\hline Total & & 20501574 & 602108 & 100.000 & 100.000 \\
\hline
\end{tabular}

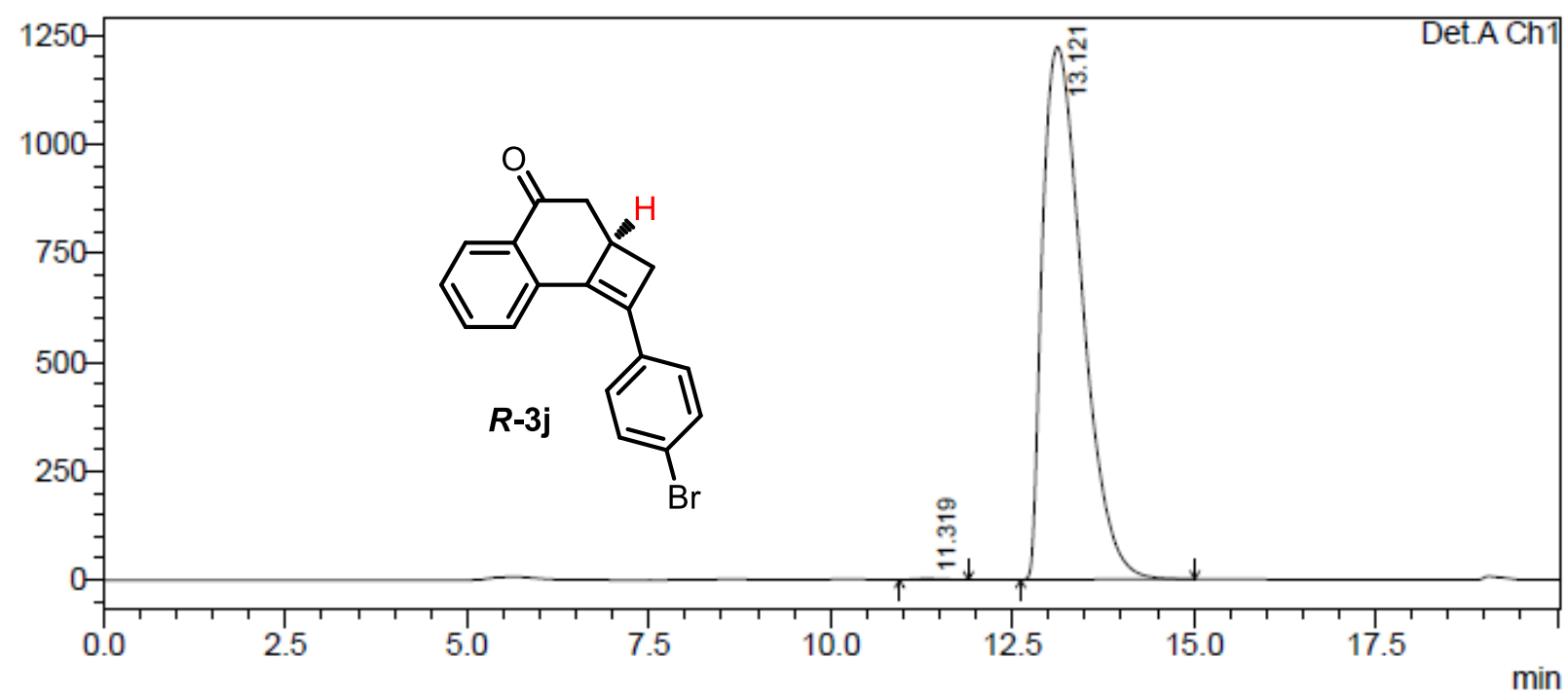

\begin{tabular}{|r|r|r|r|r|r|}
\hline \multicolumn{1}{|c|}{ Peak\# } & Ret. Time & \multicolumn{1}{|c|}{ Area } & Height & \multicolumn{1}{|c|}{ Area \% } & Height \% \\
\hline 1 & 11.319 & 60069 & 2308 & 0.129 & 0.188 \\
\hline 2 & 13.121 & 46456382 & 1222994 & 99.871 & 99.812 \\
\hline Total & & 46516451 & 1225302 & 100.000 & 100.000 \\
\hline
\end{tabular}




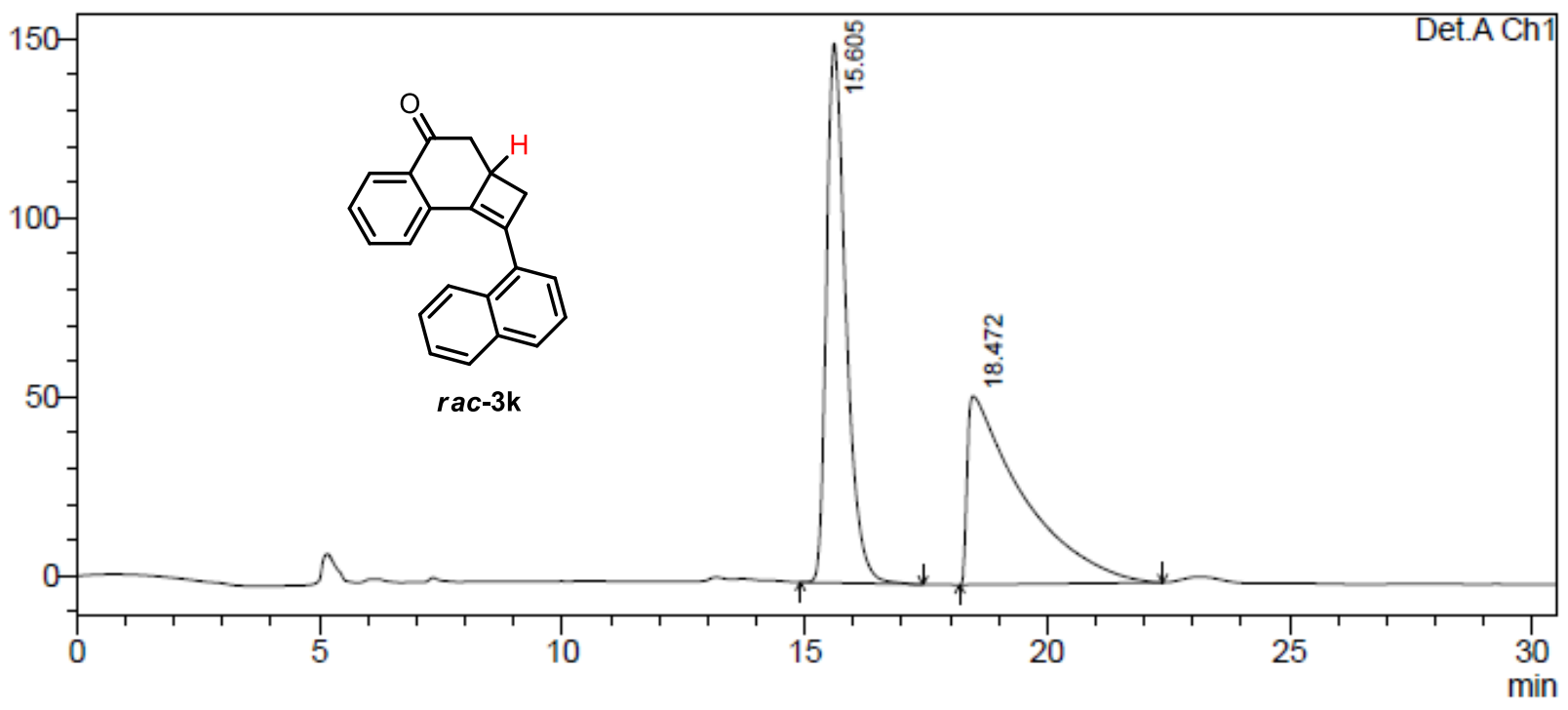

\begin{tabular}{|r|r|r|r|r|r|}
\hline \multicolumn{1}{|c|}{ Peak\# } & \multicolumn{1}{|c|}{ Ret. Time } & \multicolumn{1}{c|}{ Area } & \multicolumn{1}{c|}{ Height } & \multicolumn{1}{c|}{ Area \% } & \multicolumn{1}{c|}{ Height \% } \\
\hline 1 & 15.605 & 4223254 & 150689 & 50.437 & 74.114 \\
\hline 2 & 18.472 & 4150120 & 52632 & 49.563 & 25.886 \\
\hline Total & & 8373374 & 203321 & 100.000 & 100.000 \\
\hline
\end{tabular}

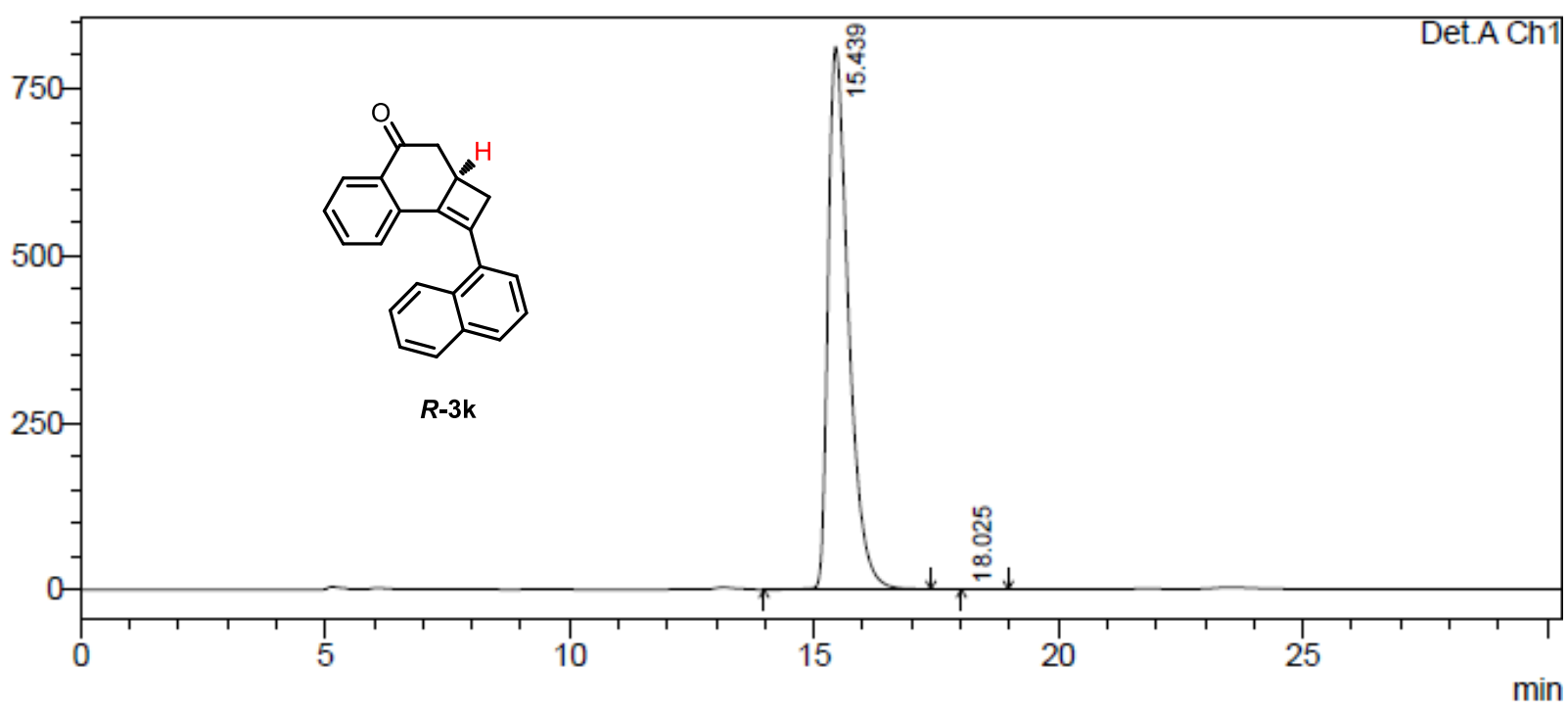

\begin{tabular}{|r|r|r|r|r|r|}
\hline \multicolumn{1}{|c|}{ Peak\# } & \multicolumn{1}{|c|}{ Ret. Time } & \multicolumn{1}{c|}{ Area } & Height & \multicolumn{1}{c|}{ Area \% } & Height \% \\
\hline 1 & 15.439 & 23619560 & 812183 & 100.002 & 100.000 \\
\hline 2 & 18.025 & -543 & -0 & -0.002 & -0.000 \\
\hline Total & & 23619016 & 812182 & 100.000 & 100.000 \\
\hline
\end{tabular}




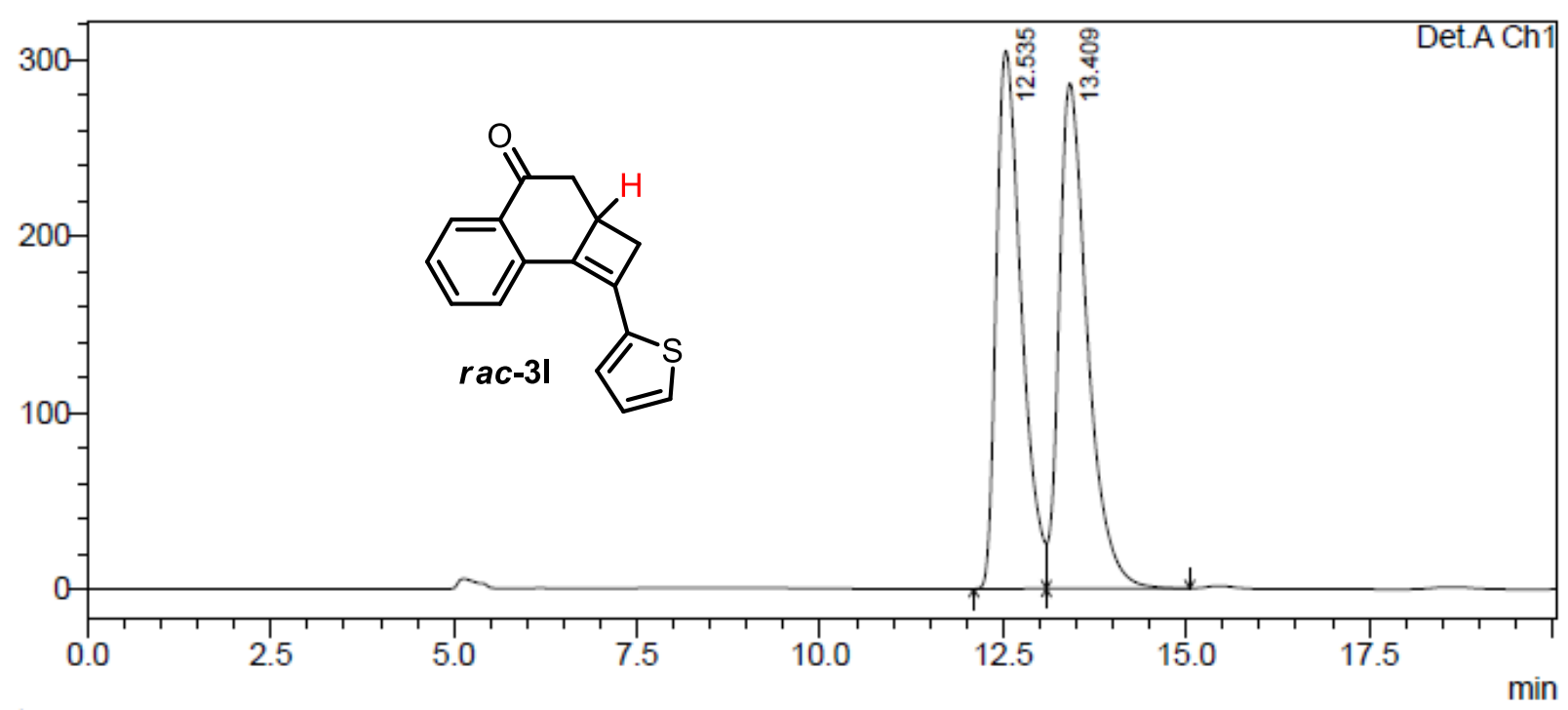

\begin{tabular}{|r|r|r|r|r|r|}
\hline Peak\# & Ret. Time & \multicolumn{1}{c|}{ Area } & Height & \multicolumn{1}{c|}{ Area \% } & \multicolumn{1}{c|}{ Height \% } \\
\hline 1 & 12.535 & 7299701 & 304896 & 48.588 & 51.564 \\
\hline 2 & 13.409 & 7723935 & 286404 & 51.412 & 48.436 \\
\hline Total & & 15023636 & 591300 & 100.000 & 100.000 \\
\hline
\end{tabular}

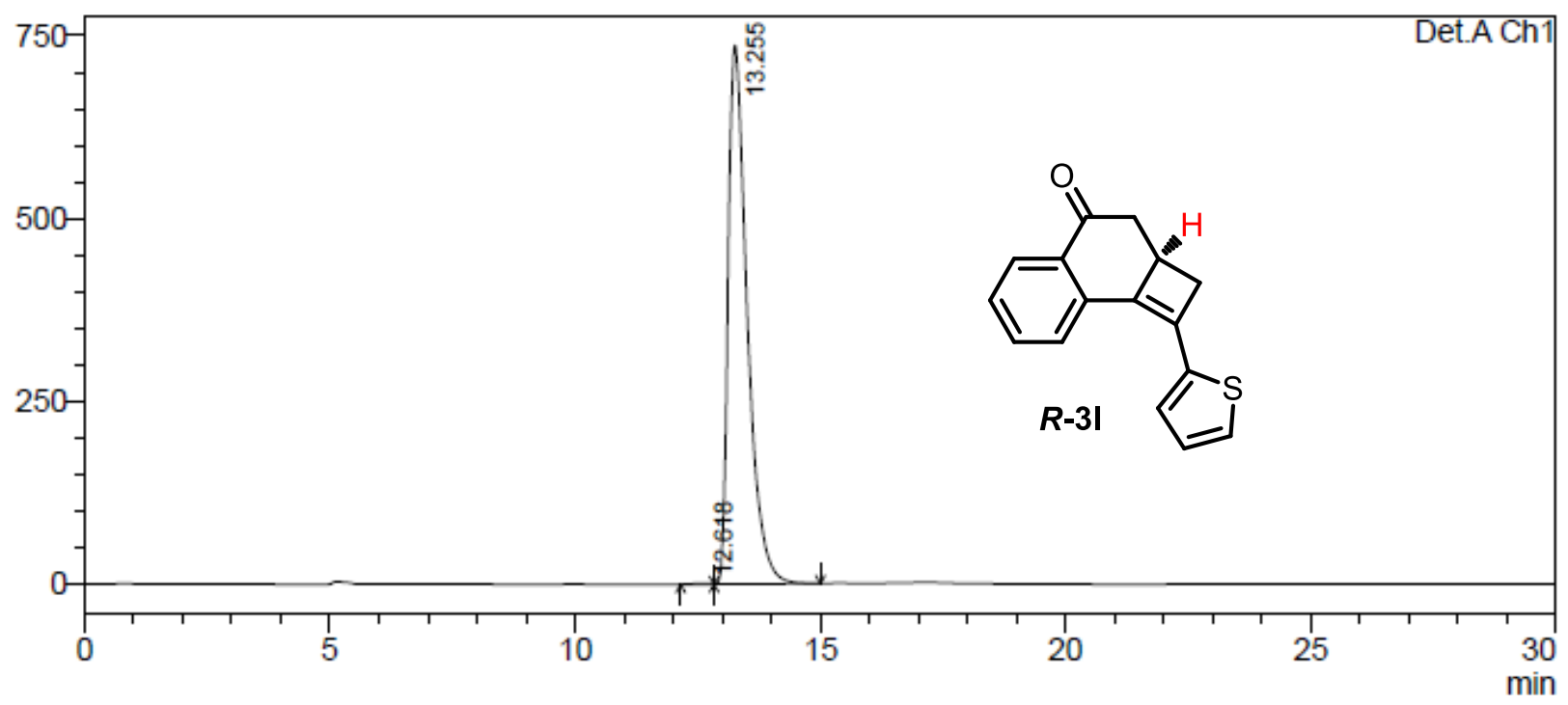

\begin{tabular}{|r|r|r|r|r|r|}
\hline \multicolumn{1}{|c|}{ Peak\# } & \multicolumn{1}{|c|}{ Ret. Time } & \multicolumn{1}{c|}{ Area } & Height & \multicolumn{1}{|c|}{ Area \% } & \multicolumn{1}{c|}{ Height \% } \\
\hline 1 & 12.618 & 36509 & 1516 & 0.183 & 0.205 \\
\hline 2 & 13.255 & 19874285 & 736899 & 99.817 & 99.795 \\
\hline Total & & 19910794 & 738415 & 100.000 & 100.000 \\
\hline
\end{tabular}



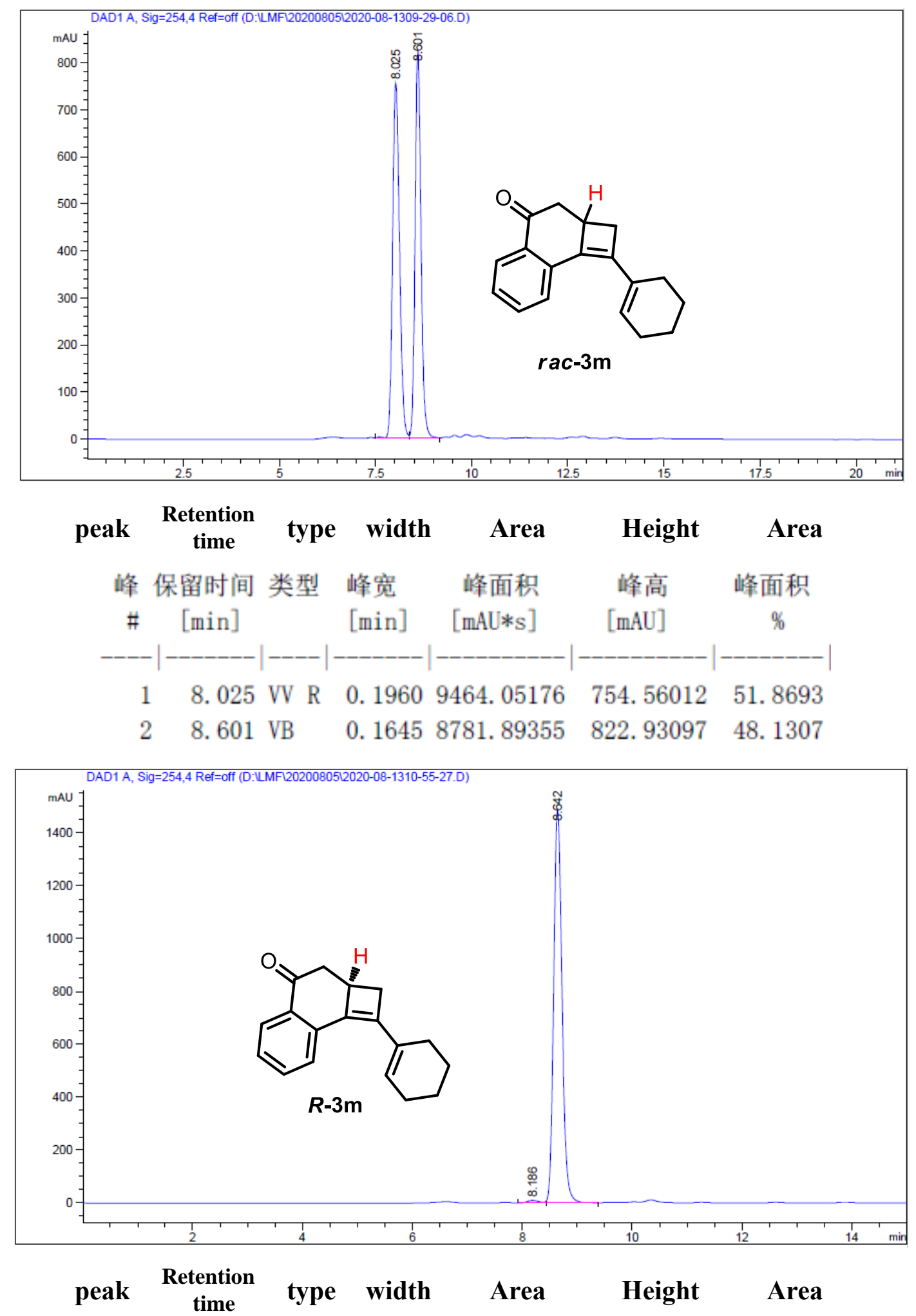

峰保留时间 类型 峰宽峰面积峰高峰面积

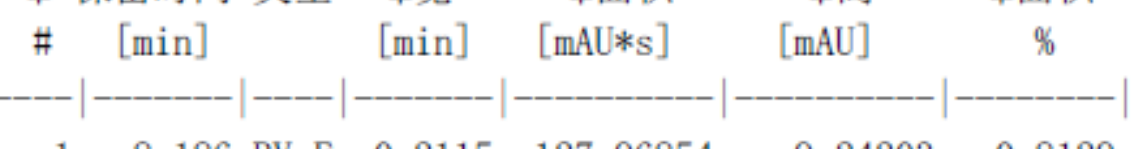
$18.186 \mathrm{BV} \mathrm{E}$
$0.2115 \quad 127.96854$
9. 24203
0.8129

28.642 VB R

0. $1625 \quad 1.56137 \mathrm{e} 4 \quad 1486.80530$

99. 1871 


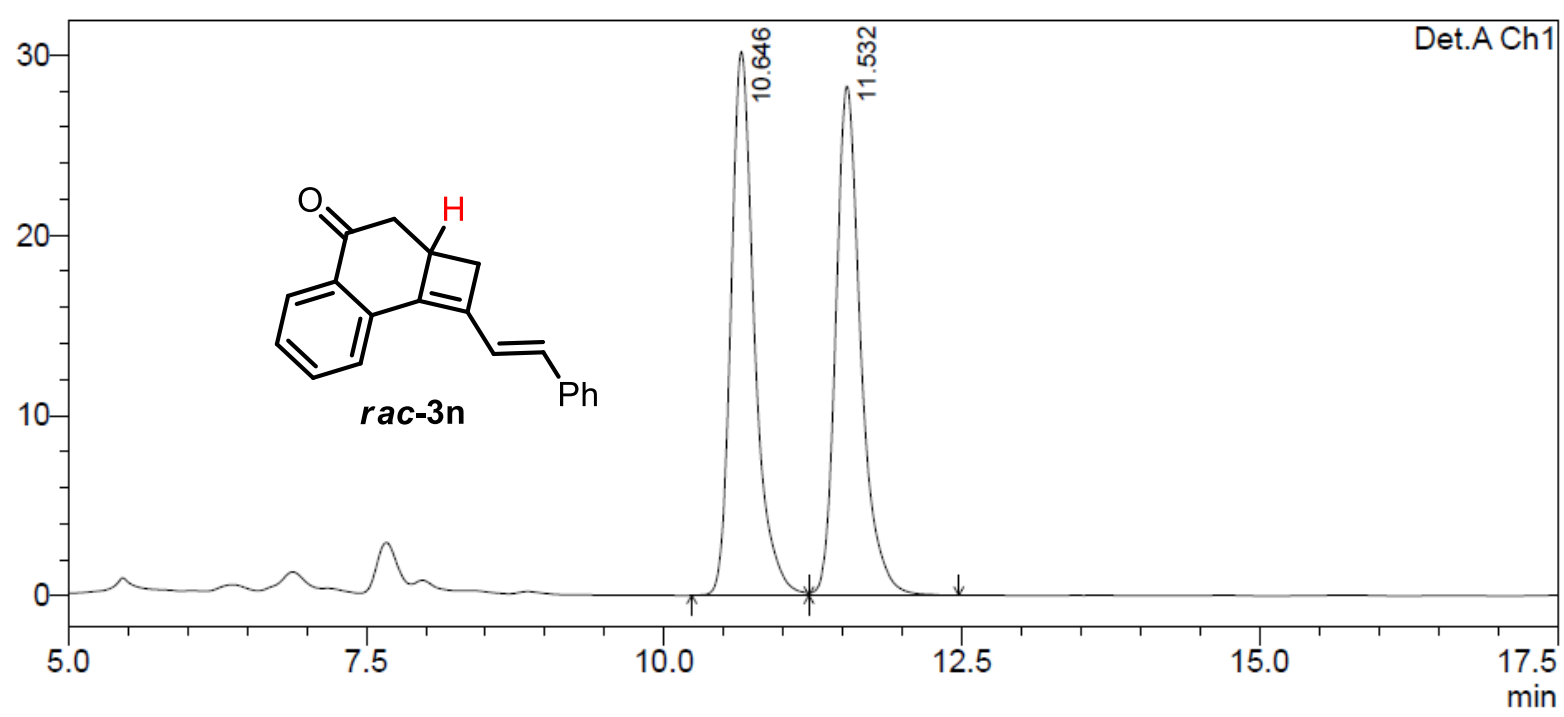

\begin{tabular}{|r|r|r|r|r|r|}
\hline \multicolumn{1}{|c|}{ Peak\# } & Ret. Time & \multicolumn{1}{c|}{ Area } & \multicolumn{1}{c|}{ Height } & \multicolumn{1}{c|}{ Area \% } & \multicolumn{1}{|c|}{ Height \% } \\
\hline 1 & 10.646 & 412663 & 30169 & 50.059 & 51.662 \\
\hline 2 & 11.532 & 411687 & 28228 & 49.941 & 48.338 \\
\hline Total & & 824350 & 58396 & 100.000 & 100.000 \\
\hline
\end{tabular}

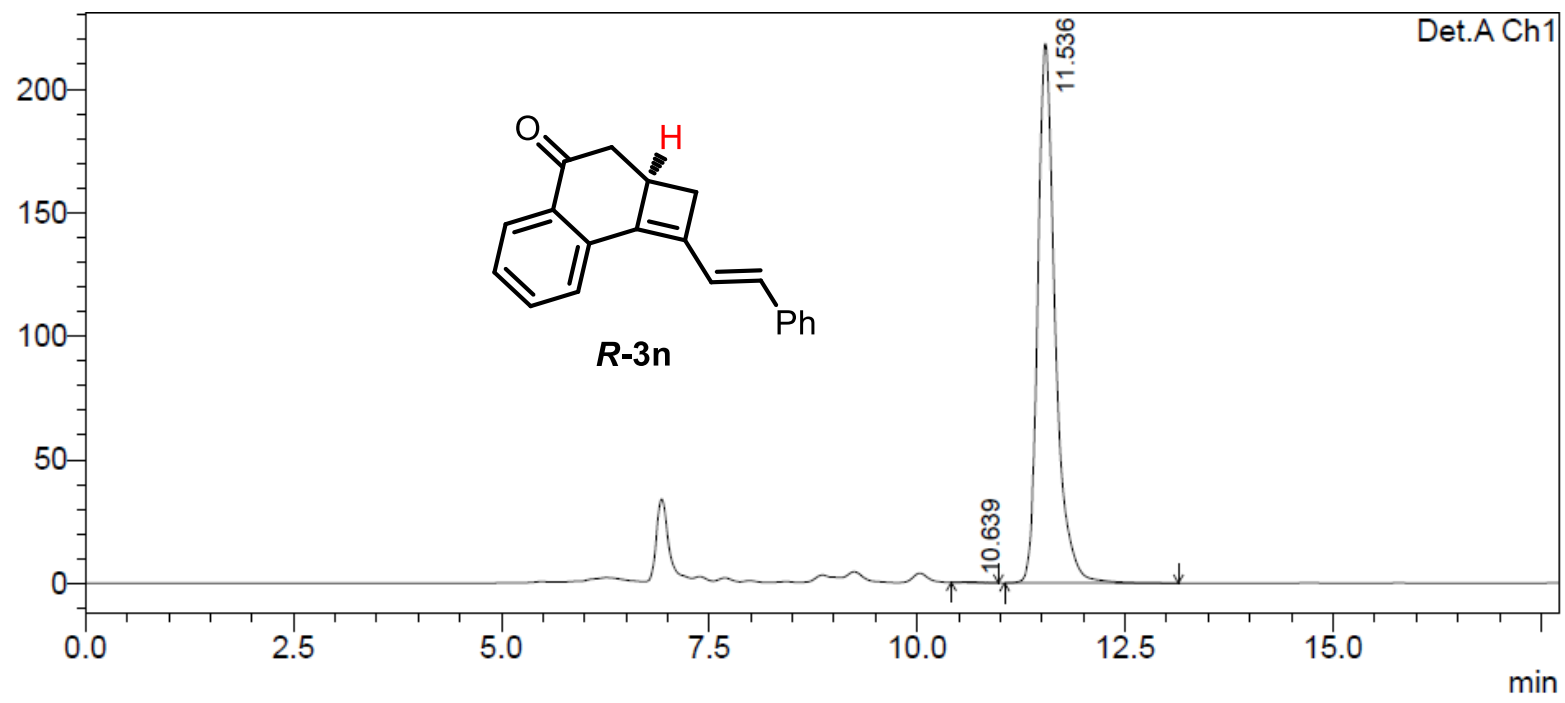

\begin{tabular}{|r|r|r|r|r|r|}
\hline \multicolumn{1}{|c|}{ Peak\# } & Ret. Time & \multicolumn{1}{c|}{ Area } & \multicolumn{1}{|c|}{ Height } & \multicolumn{1}{|c|}{ Area \% } & \multicolumn{1}{c|}{ Height \% } \\
\hline 1 & 10.639 & 3595 & 263 & 0.112 & 0.120 \\
\hline 2 & 11.536 & 3198923 & 218363 & 99.888 & 99.880 \\
\hline Total & & 3202517 & 218626 & 100.000 & 100.000 \\
\hline
\end{tabular}




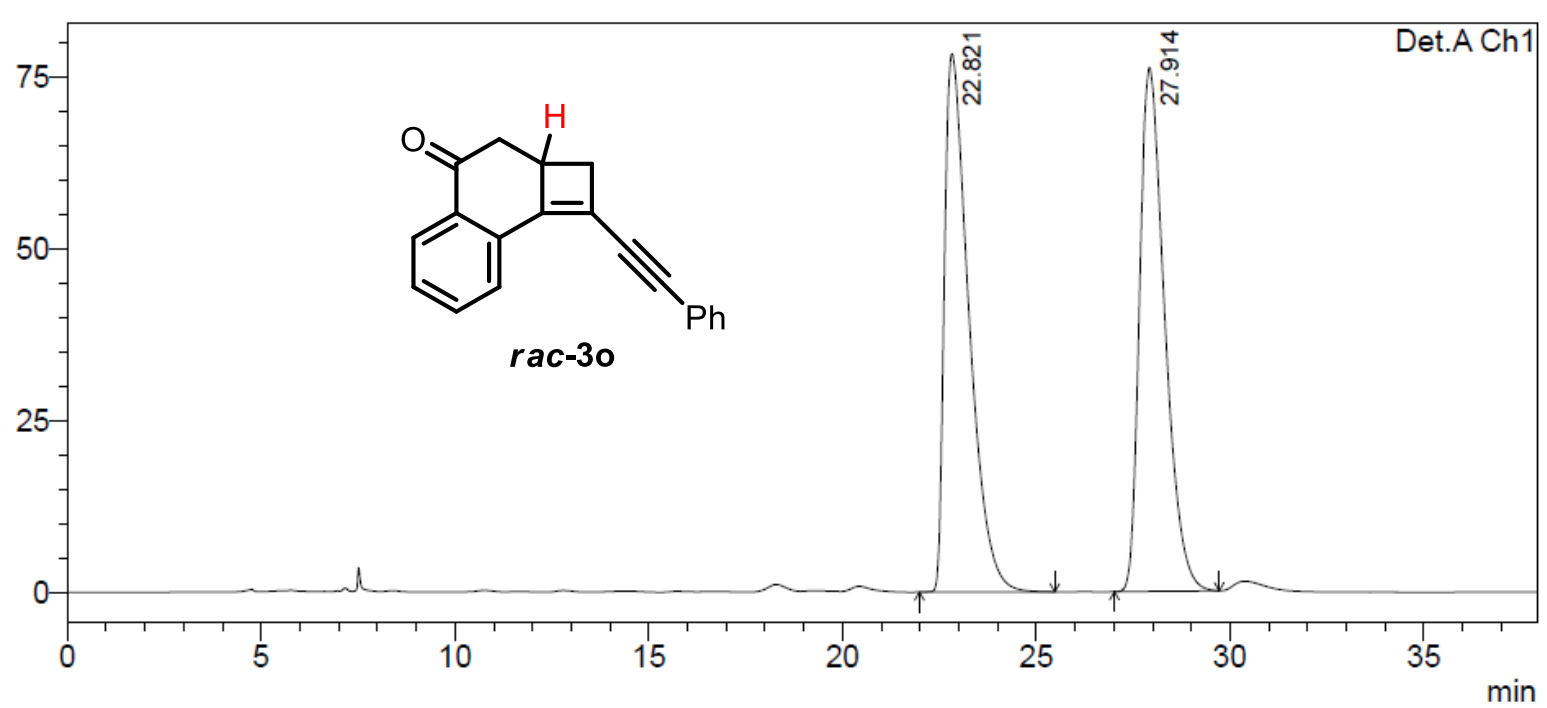

\begin{tabular}{|r|r|r|r|r|r|}
\hline Peak\# & Ret. Time & \multicolumn{1}{|c|}{ Area } & \multicolumn{1}{|c|}{ Height } & \multicolumn{1}{|c|}{ Area \% } & \multicolumn{1}{|c|}{ Height \% } \\
\hline 1 & 22.821 & 3448569 & 78377 & 50.120 & 50.686 \\
\hline 2 & 27.914 & 3432106 & 76254 & 49.880 & 49.314 \\
\hline Total & & 6880675 & 154630 & 100.000 & 100.000 \\
\hline
\end{tabular}

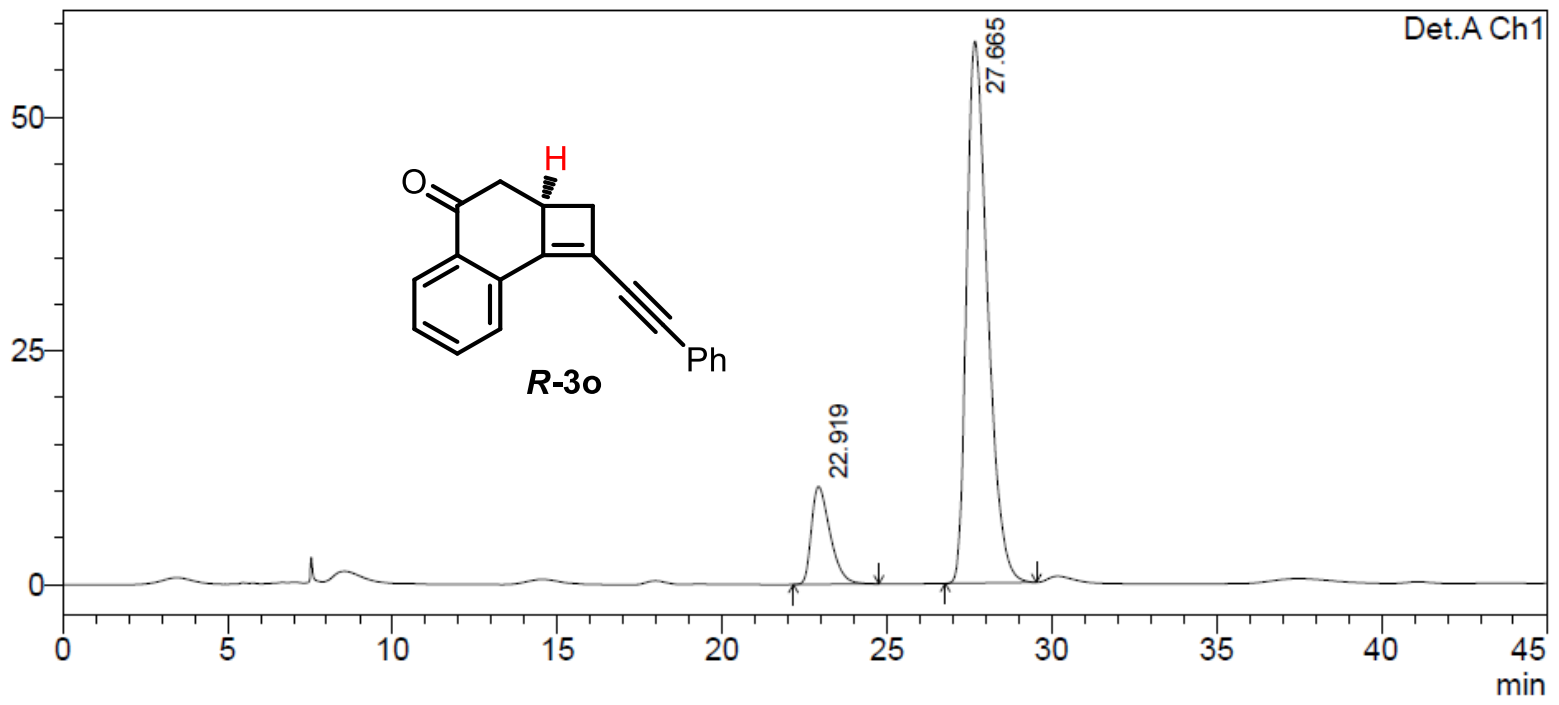

\begin{tabular}{|r|r|r|r|r|r|}
\hline \multicolumn{1}{|c|}{ Peak\# } & Ret. Time & \multicolumn{1}{c|}{ Area } & \multicolumn{1}{c|}{ Height } & \multicolumn{1}{c|}{ Area \% } & \multicolumn{1}{c|}{ Height $\%$} \\
\hline 1 & 22.919 & 413906 & 10454 & 13.880 & 15.287 \\
\hline 2 & 27.665 & 2568128 & 57931 & 86.120 & 84.713 \\
\hline Total & & 2982034 & 68385 & 100.000 & 100.000 \\
\hline
\end{tabular}




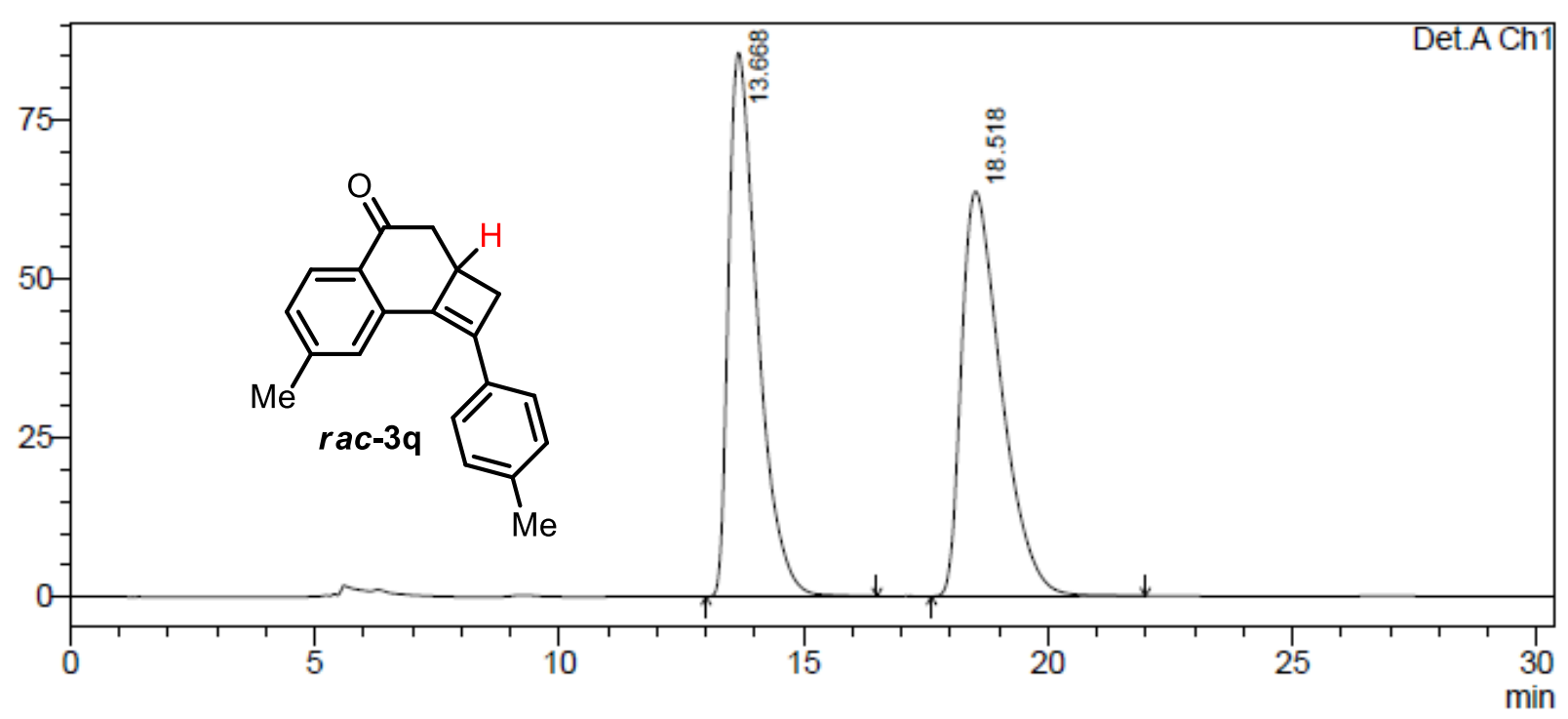

\begin{tabular}{|r|r|r|r|r|r|}
\hline \multicolumn{1}{|c|}{ Peak\# } & Ret. Time & \multicolumn{1}{|c|}{ Area } & \multicolumn{1}{|c|}{ Height } & \multicolumn{1}{|c|}{ Area \% } & \multicolumn{1}{c|}{ Height \% } \\
\hline 1 & 13.668 & 3554322 & 85501 & 49.632 & 57.331 \\
\hline 2 & 18.518 & 3607040 & 63635 & 50.368 & 42.669 \\
\hline Total & & 7161362 & 149136 & 100.000 & 100.000 \\
\hline
\end{tabular}

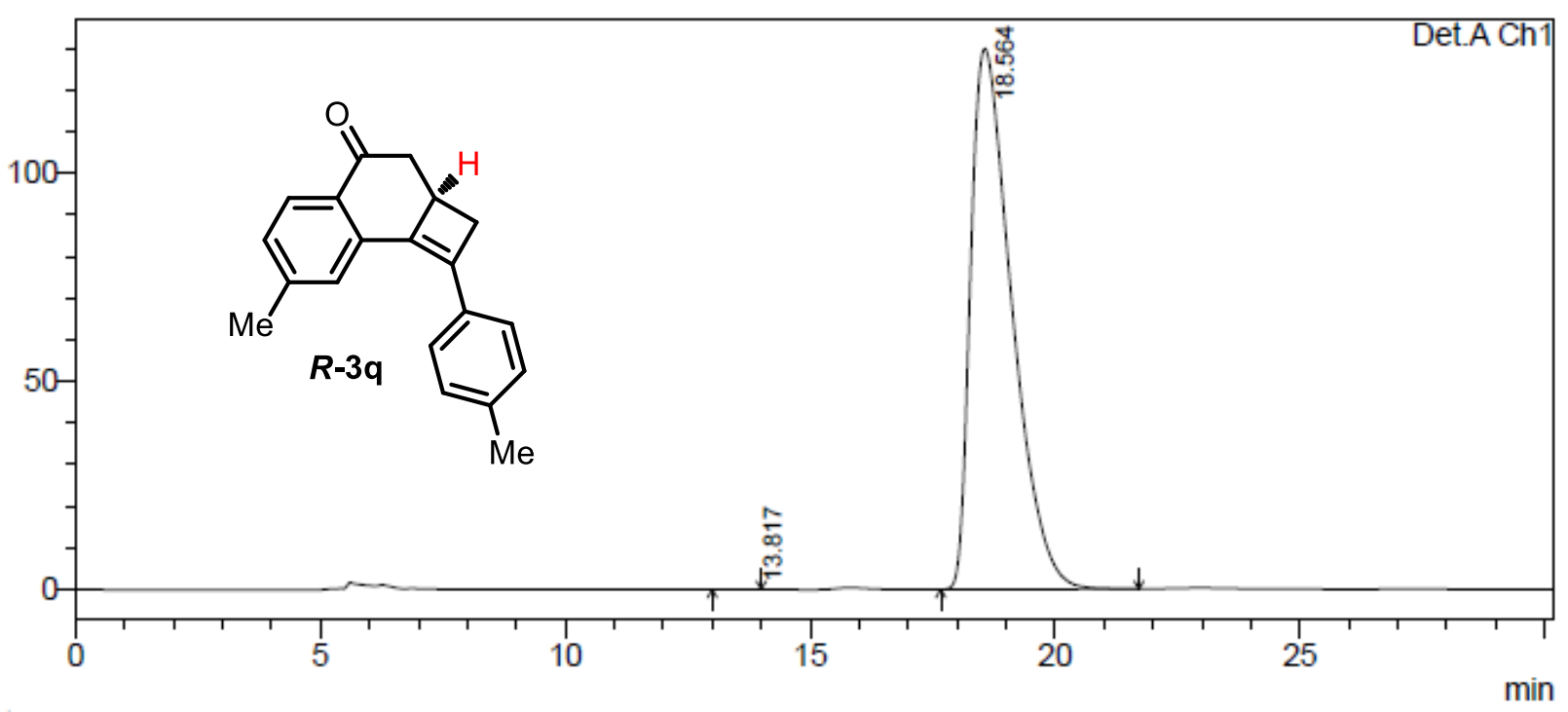

\begin{tabular}{|r|r|r|r|r|r|}
\hline \multicolumn{1}{|c|}{ Peak\# } & \multicolumn{1}{|c|}{ Ret. Time } & \multicolumn{1}{|c|}{ Area } & \multicolumn{1}{c|}{ Height } & \multicolumn{1}{c|}{ Area \% } & \multicolumn{1}{c|}{ Height \% } \\
\hline 1 & 13.817 & 323 & 26 & 0.004 & 0.020 \\
\hline 2 & 18.564 & 7600750 & 129999 & 99.996 & 99.980 \\
\hline Total & & 7601073 & 130025 & 100.000 & 100.000 \\
\hline
\end{tabular}




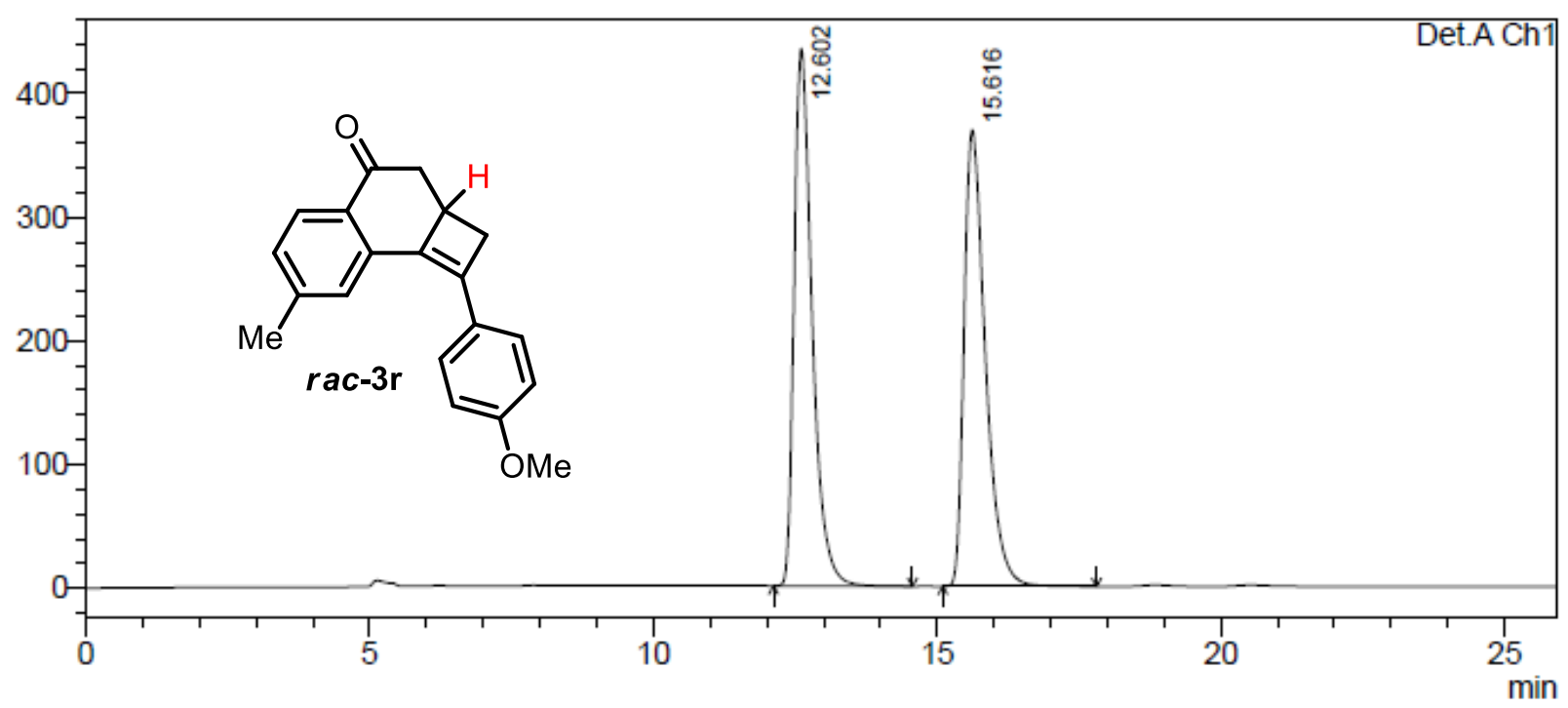

\begin{tabular}{|r|r|r|r|r|r|}
\hline Peak\# & Ret. Time & \multicolumn{1}{|c|}{ Area } & \multicolumn{1}{|c|}{ Height } & \multicolumn{1}{|c|}{ Area \% } & \multicolumn{1}{c|}{ Height \% } \\
\hline 1 & 12.602 & 9616317 & 434413 & 50.039 & 54.074 \\
\hline 2 & 15.616 & 9601176 & 368948 & 49.961 & 45.926 \\
\hline Total & & 19217492 & 803361 & 100.000 & 100.000 \\
\hline
\end{tabular}

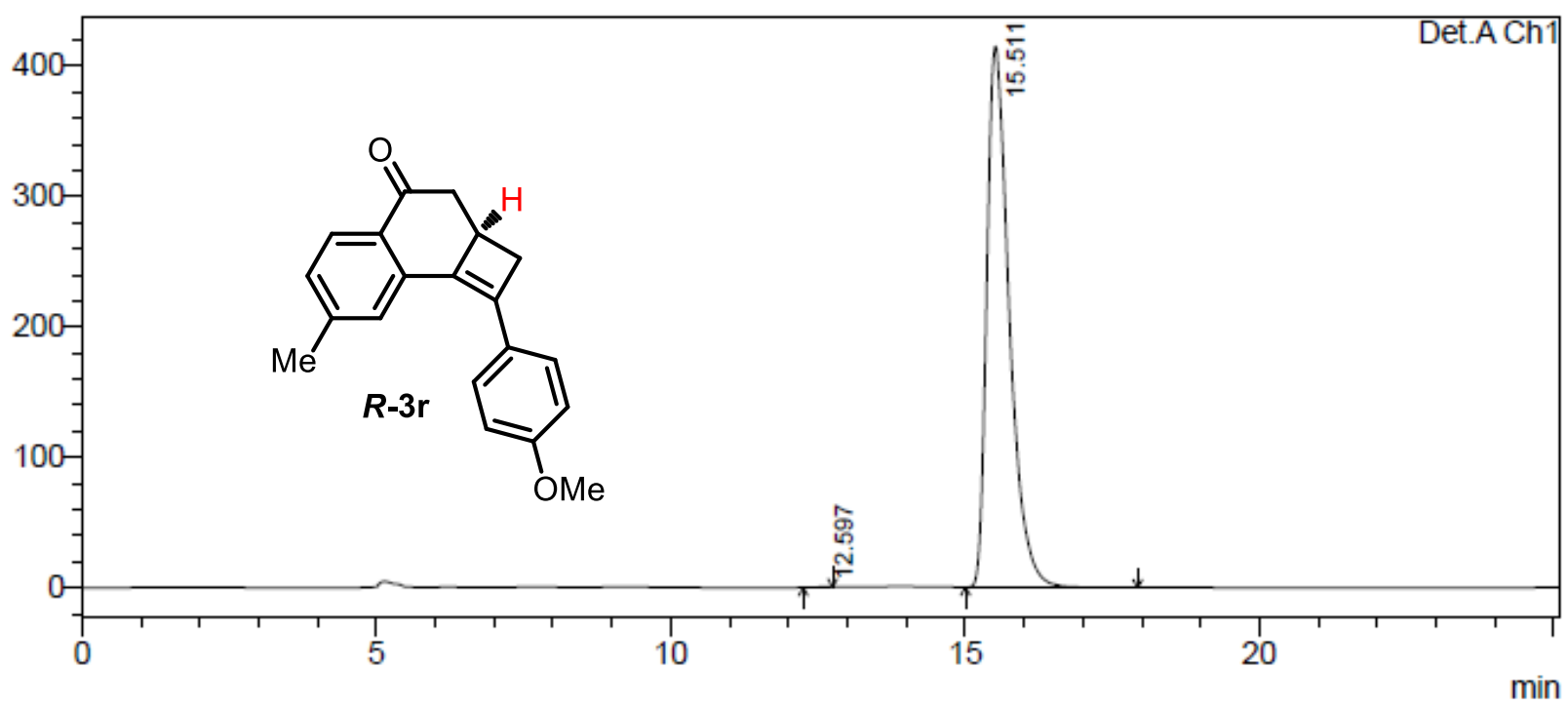

\begin{tabular}{|r|r|r|r|r|r|}
\hline \multicolumn{1}{|c|}{ Peak\# } & \multicolumn{1}{|c|}{ Ret. Time } & \multicolumn{1}{c|}{ Area } & \multicolumn{1}{c|}{ Height } & \multicolumn{1}{c|}{ Area \% } & \multicolumn{1}{c|}{ Height \% } \\
\hline 1 & 12.597 & 2545 & 156 & 0.023 & 0.038 \\
\hline 2 & 15.511 & 11015633 & 414672 & 99.977 & 99.962 \\
\hline Total & & 11018179 & 414828 & 100.000 & 100.000 \\
\hline
\end{tabular}




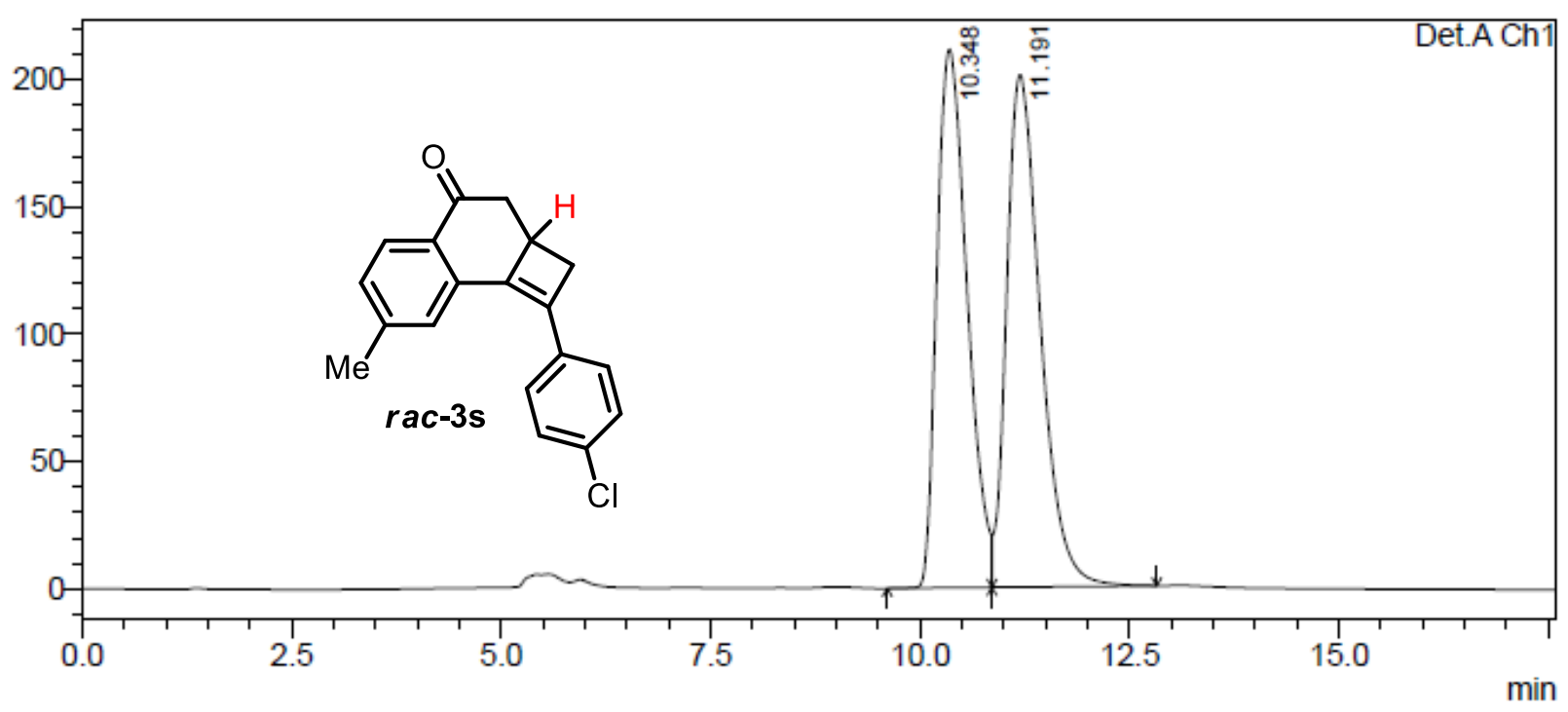

\begin{tabular}{|r|r|r|r|r|r|}
\hline \multicolumn{1}{|c|}{ Peak\# } & \multicolumn{1}{|c|}{ Ret. Time } & \multicolumn{1}{c|}{ Area } & \multicolumn{1}{c|}{ Height } & \multicolumn{1}{c|}{ Area \% } & \multicolumn{1}{c|}{ Height \% } \\
\hline 1 & 10.348 & 5325158 & 211553 & 48.526 & 51.247 \\
\hline 2 & 11.191 & 5648660 & 201257 & 51.474 & 48.753 \\
\hline Total & & 10973817 & 412810 & 100.000 & 100.000 \\
\hline
\end{tabular}

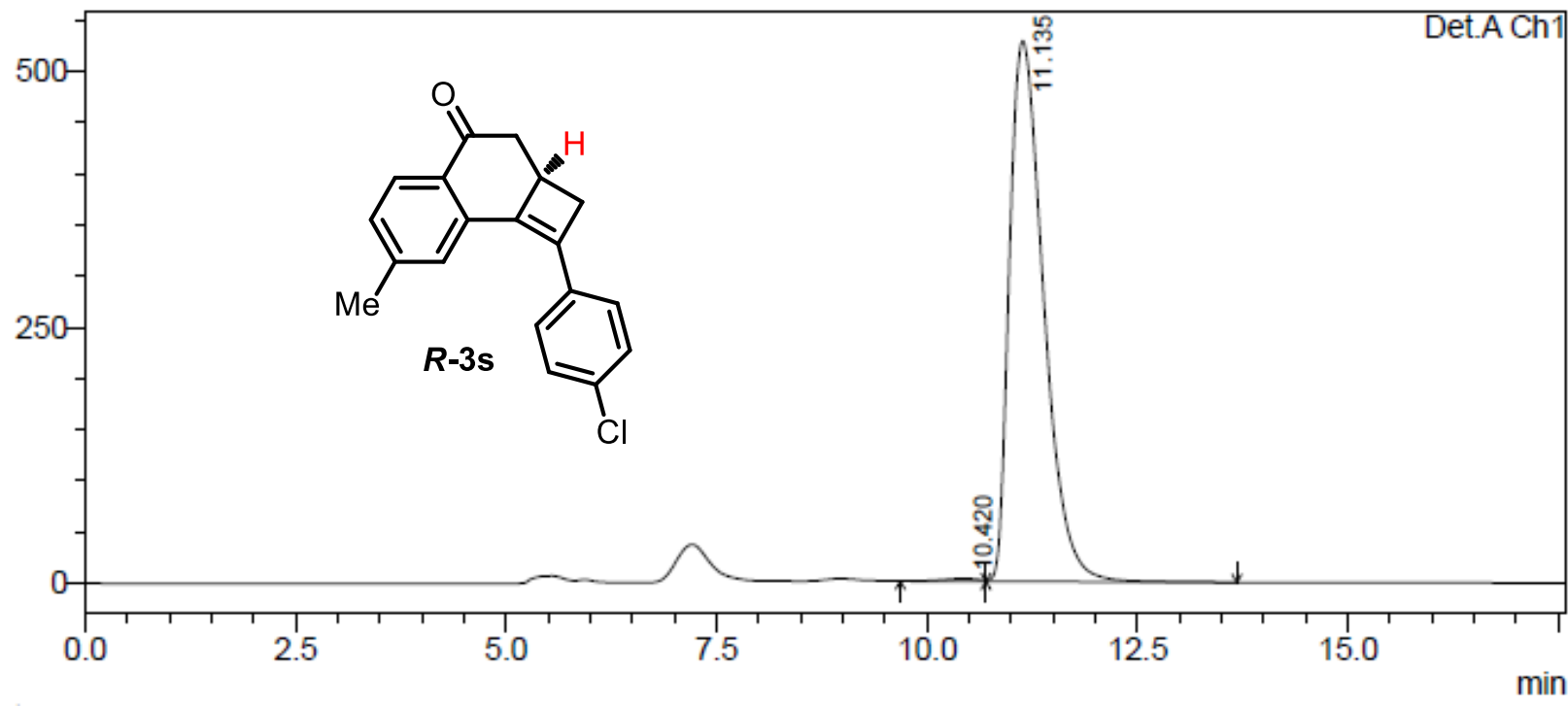

\begin{tabular}{|r|r|r|r|r|r|}
\hline \multicolumn{1}{|c|}{ Peak\# } & Ret. Time & \multicolumn{1}{c|}{ Area } & \multicolumn{1}{|c|}{ Height } & \multicolumn{1}{|c|}{ Area \% } & \multicolumn{1}{c|}{ Height \% } \\
\hline 1 & 10.420 & 82741 & 2647 & 0.551 & 0.499 \\
\hline 2 & 11.135 & 14938399 & 528316 & 99.449 & 99.501 \\
\hline Total & & 15021139 & 530964 & 100.000 & 100.000 \\
\hline
\end{tabular}




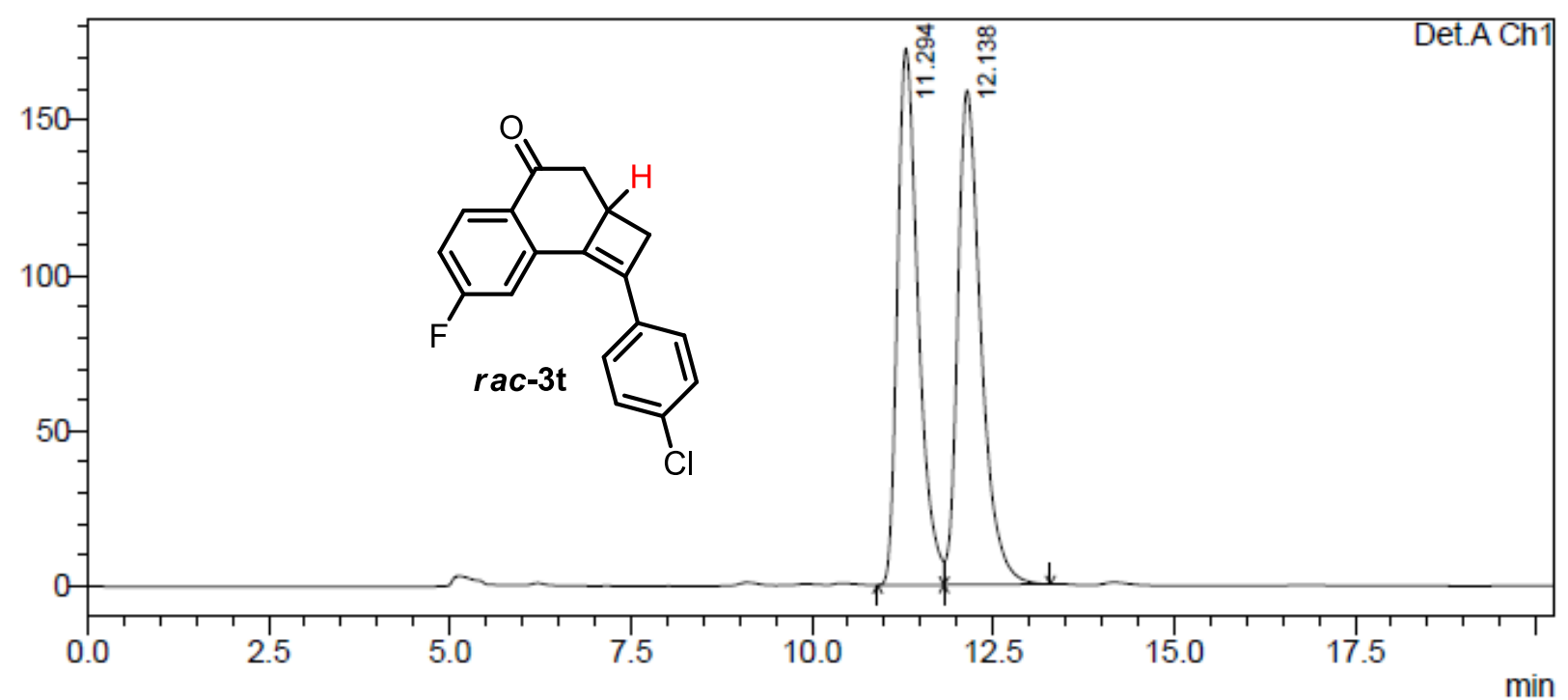

\begin{tabular}{|r|r|r|r|r|r|}
\hline Peak\# & Ret. Time & \multicolumn{1}{c|}{ Area } & Height & \multicolumn{1}{c|}{ Area \% } & Height \% \\
\hline 1 & 11.294 & 3452913 & 172506 & 49.209 & 52.060 \\
\hline 2 & 12.138 & 3563980 & 158853 & 50.791 & 47.940 \\
\hline Total & & 7016893 & 331359 & 100.000 & 100.000 \\
\hline
\end{tabular}

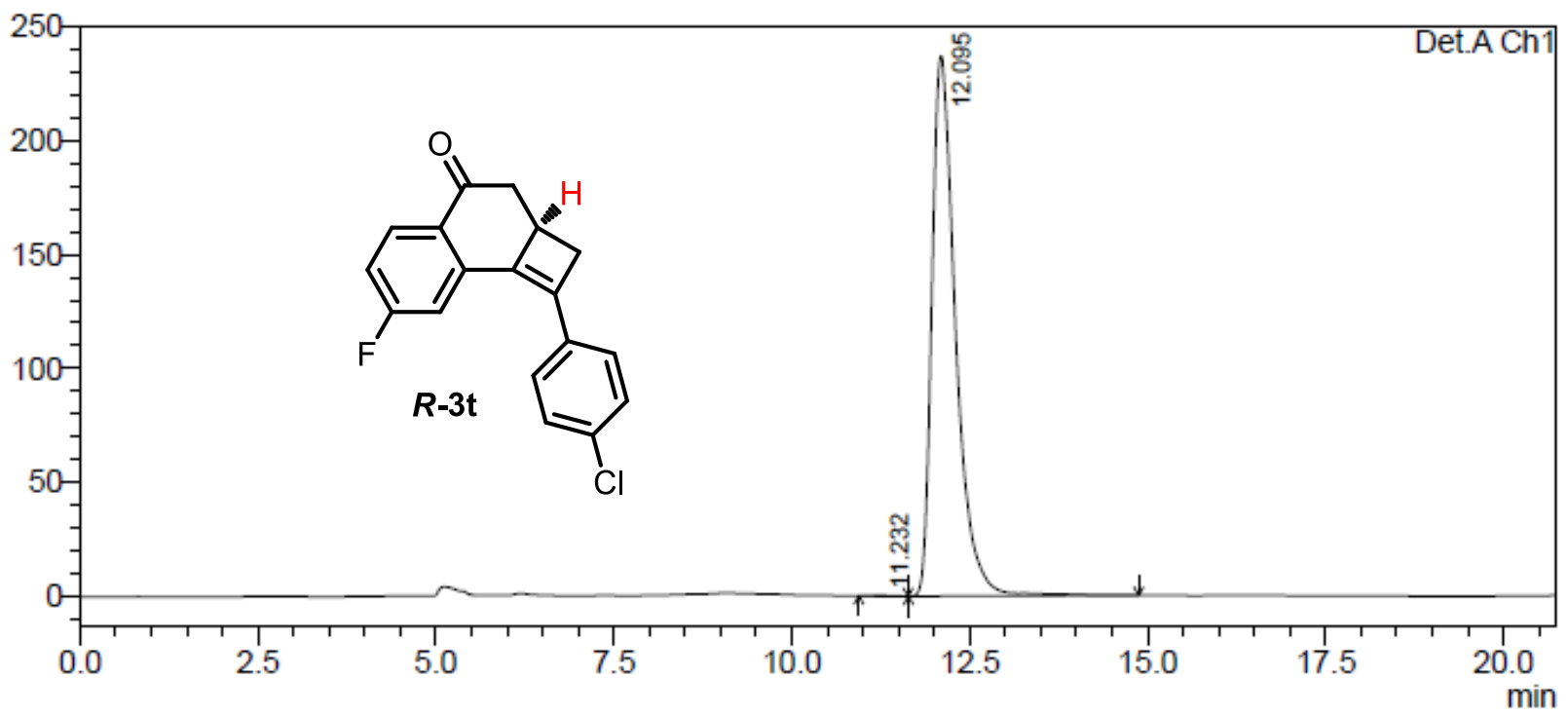

\begin{tabular}{|r|r|r|r|r|r|}
\hline \multicolumn{1}{|c|}{ Peak\# } & \multicolumn{1}{|c|}{ Ret. Time } & \multicolumn{1}{|c|}{ Area } & \multicolumn{1}{c|}{ Height } & \multicolumn{1}{c|}{ Area \% } & \multicolumn{1}{c|}{ Height \% } \\
\hline 1 & 11.232 & 5894 & 331 & 0.107 & 0.140 \\
\hline 2 & 12.095 & 5489448 & 236663 & 99.893 & 99.860 \\
\hline Total & & 5495342 & 236995 & 100.000 & 100.000 \\
\hline
\end{tabular}




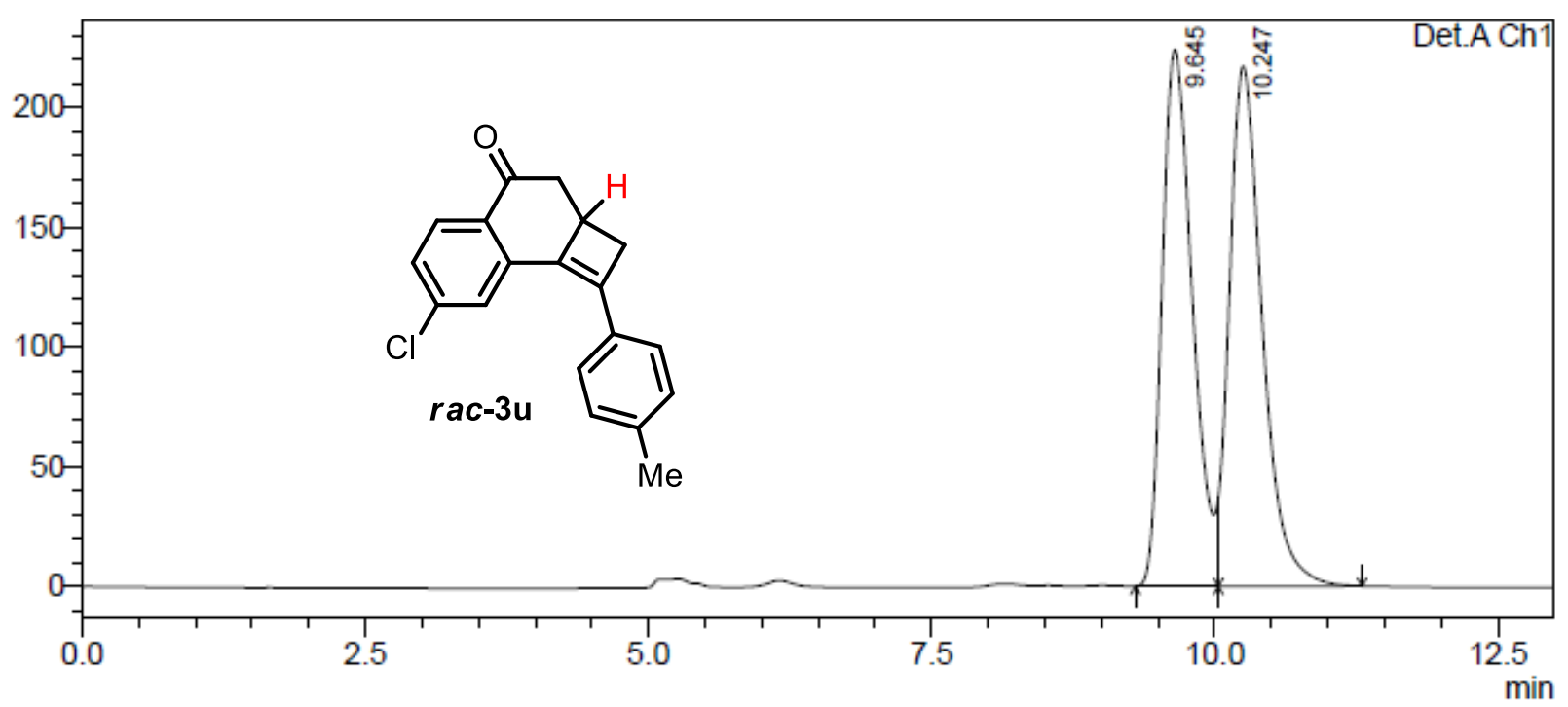

\begin{tabular}{|r|r|r|r|r|r|}
\hline \multicolumn{1}{|c|}{ Peak\# } & \multicolumn{1}{|c|}{ Ret. Time } & \multicolumn{1}{c|}{ Area } & Height & \multicolumn{1}{c|}{ Area \% } & \multicolumn{1}{c|}{ Height \% } \\
\hline 1 & 9.645 & 4204578 & 224243 & 49.090 & 50.794 \\
\hline 2 & 10.247 & 4360488 & 217229 & 50.910 & 49.206 \\
\hline Total & & 8565066 & 441472 & 100.000 & 100.000 \\
\hline
\end{tabular}

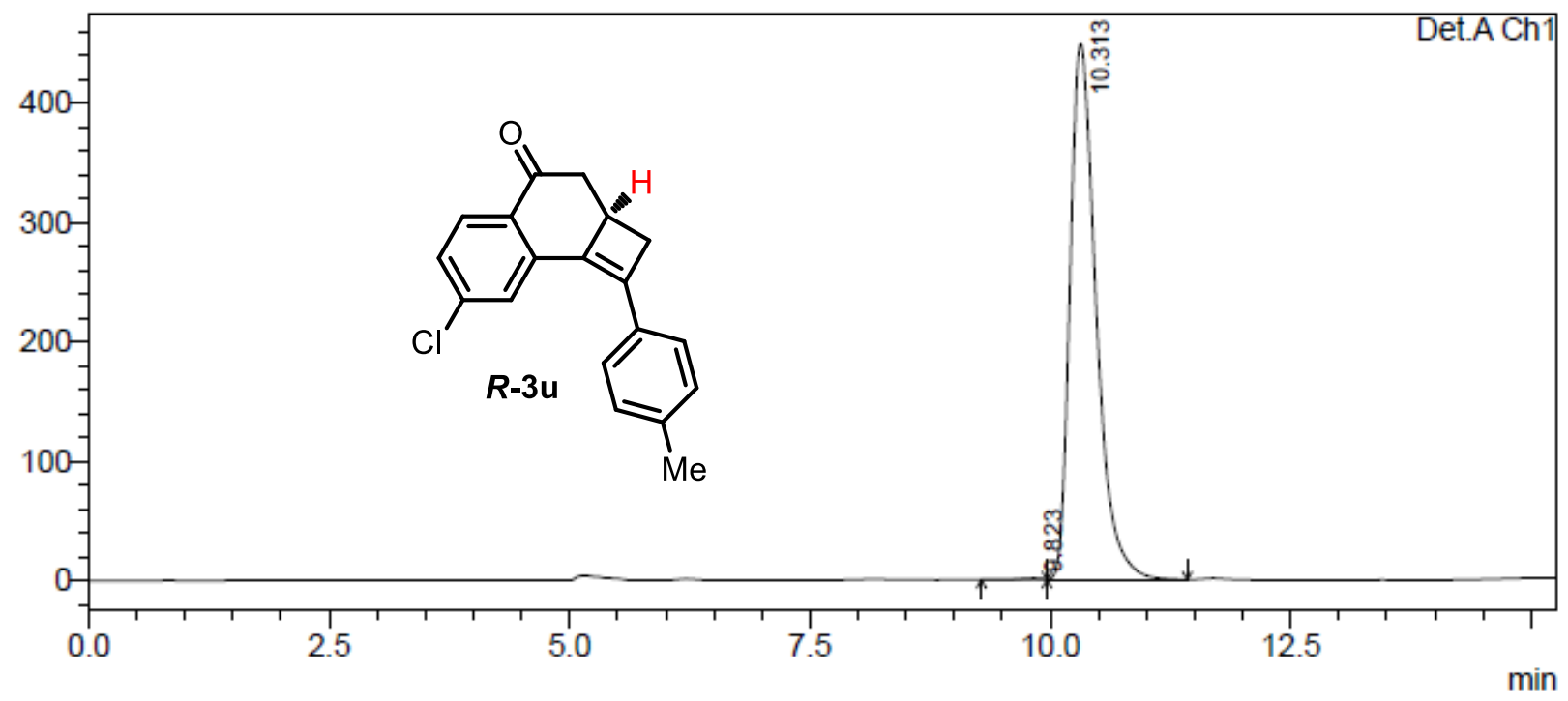

\begin{tabular}{|r|r|r|r|r|r|}
\hline \multicolumn{1}{|c|}{ Peak\# } & \multicolumn{1}{|c|}{ Ret. Time } & \multicolumn{1}{|c|}{ Area } & \multicolumn{1}{|c|}{ Height } & \multicolumn{1}{c|}{ Area \% } & \multicolumn{1}{c|}{ Height \% } \\
\hline 1 & 9.823 & 20984 & 1263 & 0.246 & 0.280 \\
\hline 2 & 10.313 & 8508354 & 449361 & 99.754 & 99.720 \\
\hline Total & & 8529337 & 450624 & 100.000 & 100.000 \\
\hline
\end{tabular}




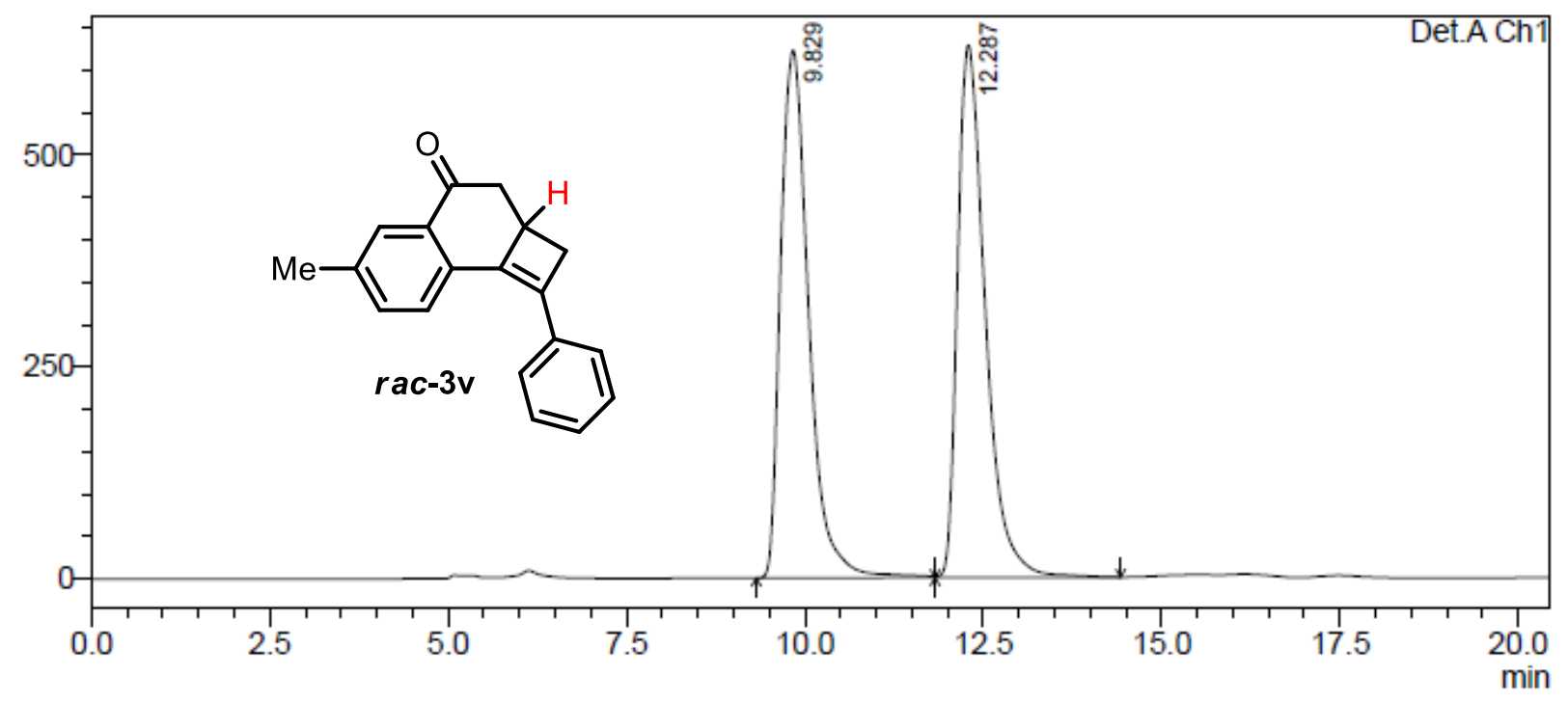

\begin{tabular}{|r|r|r|r|r|r|}
\hline \multicolumn{1}{|c|}{ Peak\# } & \multicolumn{1}{|c|}{ Ret. Time } & \multicolumn{1}{c|}{ Area } & Height & \multicolumn{1}{c|}{ Area \% } & \multicolumn{1}{c|}{ Height \% } \\
\hline 1 & 9.829 & 17100649 & 622467 & 50.164 & 49.786 \\
\hline 2 & 12.287 & 16988919 & 627812 & 49.836 & 50.214 \\
\hline Total & & 34089568 & 1250278 & 100.000 & 100.000 \\
\hline
\end{tabular}

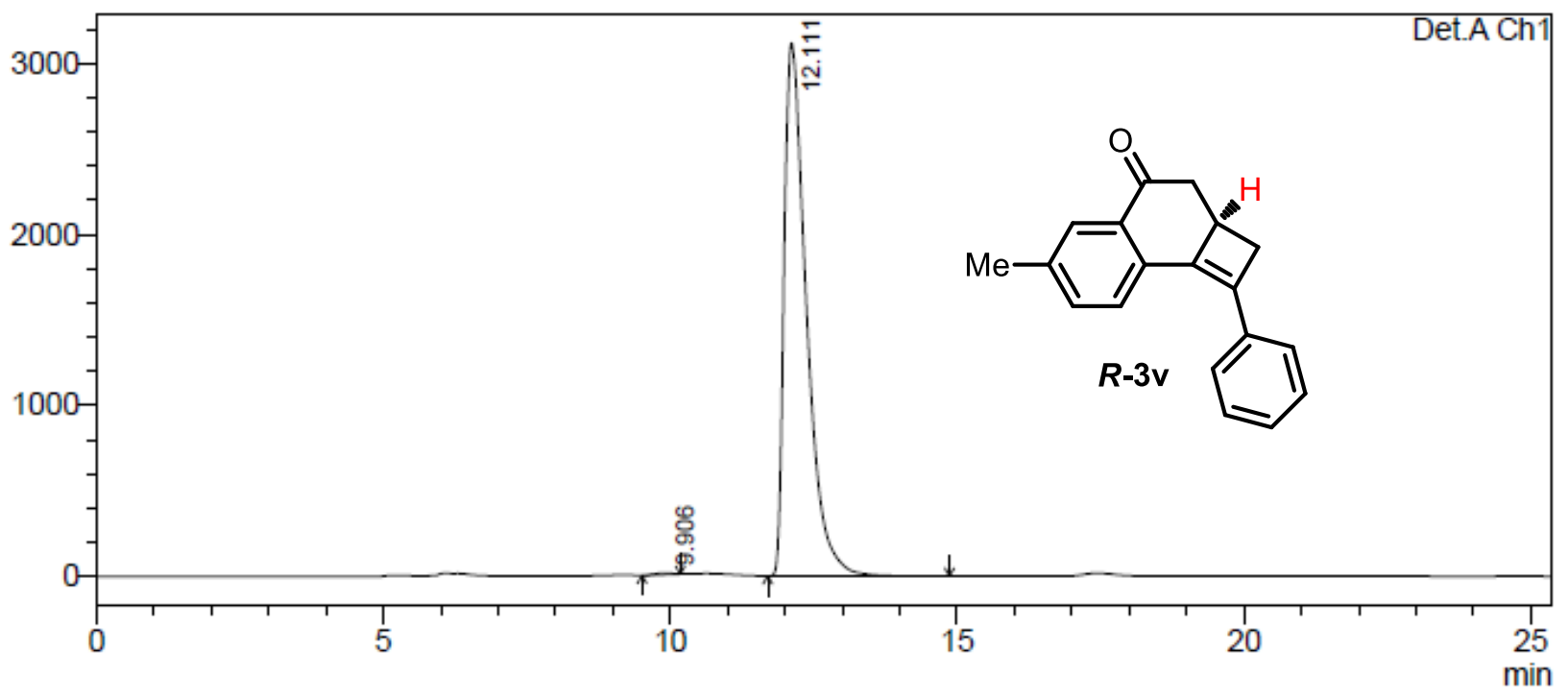

\begin{tabular}{|r|r|r|r|r|r|}
\hline \multicolumn{1}{|c|}{ Peak\# } & \multicolumn{1}{|c|}{ Ret. Time } & \multicolumn{1}{c|}{ Area } & Height & \multicolumn{1}{c|}{ Area \% } & \multicolumn{1}{c|}{ Height \% } \\
\hline 1 & 9.906 & 195221 & 9812 & 0.232 & 0.314 \\
\hline 2 & 12.111 & 83993708 & 3114908 & 99.768 & 99.686 \\
\hline Total & & 84188929 & 3124720 & 100.000 & 100.000 \\
\hline
\end{tabular}




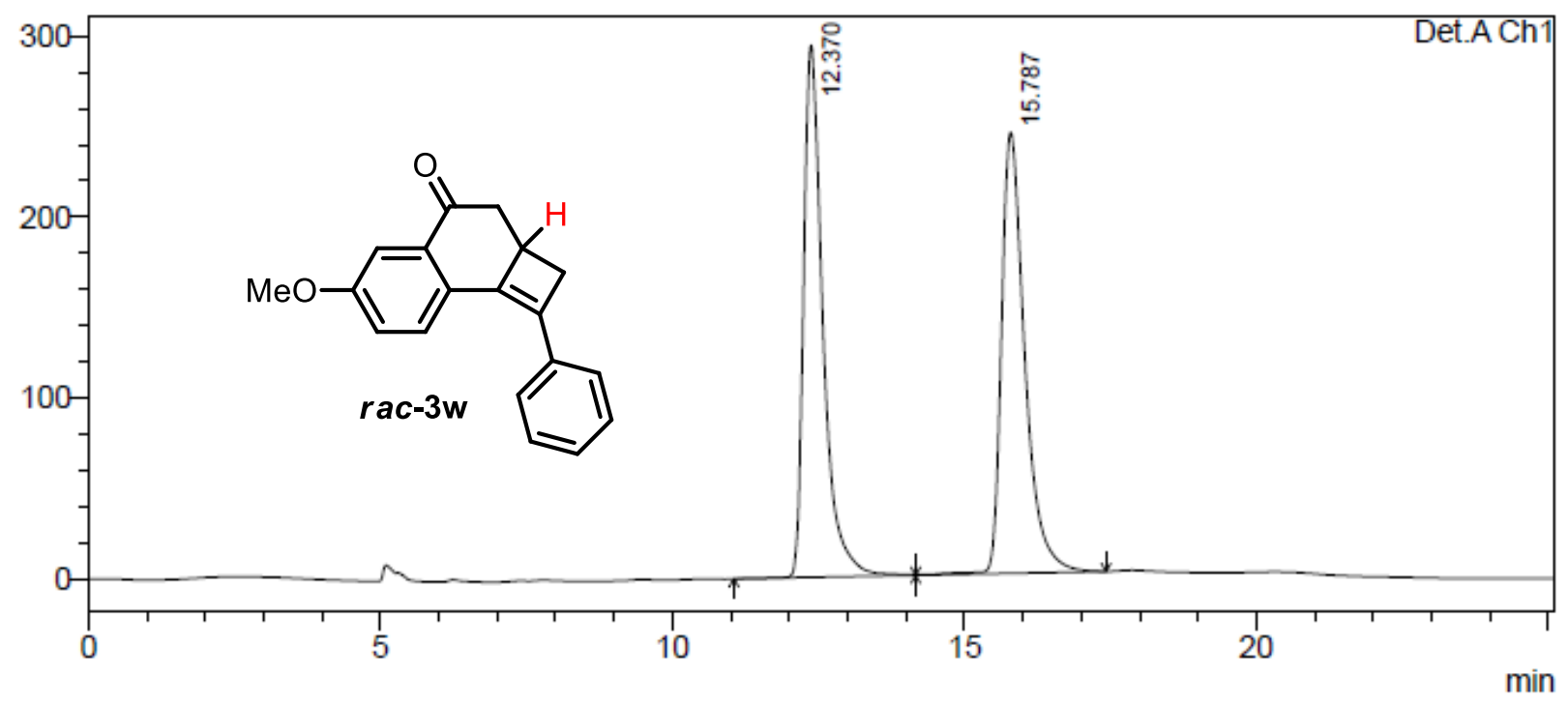

\begin{tabular}{|r|r|r|r|r|r|}
\hline \multicolumn{1}{|c|}{ Peak\# } & Ret. Time & \multicolumn{1}{|c|}{ Area } & Height & \multicolumn{1}{|c|}{ Area \% } & \multicolumn{1}{c|}{ Height \% } \\
\hline 1 & 12.370 & 6754627 & 293750 & 49.970 & 54.672 \\
\hline 2 & 15.787 & 6762643 & 243544 & 50.030 & 45.328 \\
\hline Total & & 13517271 & 537294 & 100.000 & 100.000 \\
\hline
\end{tabular}

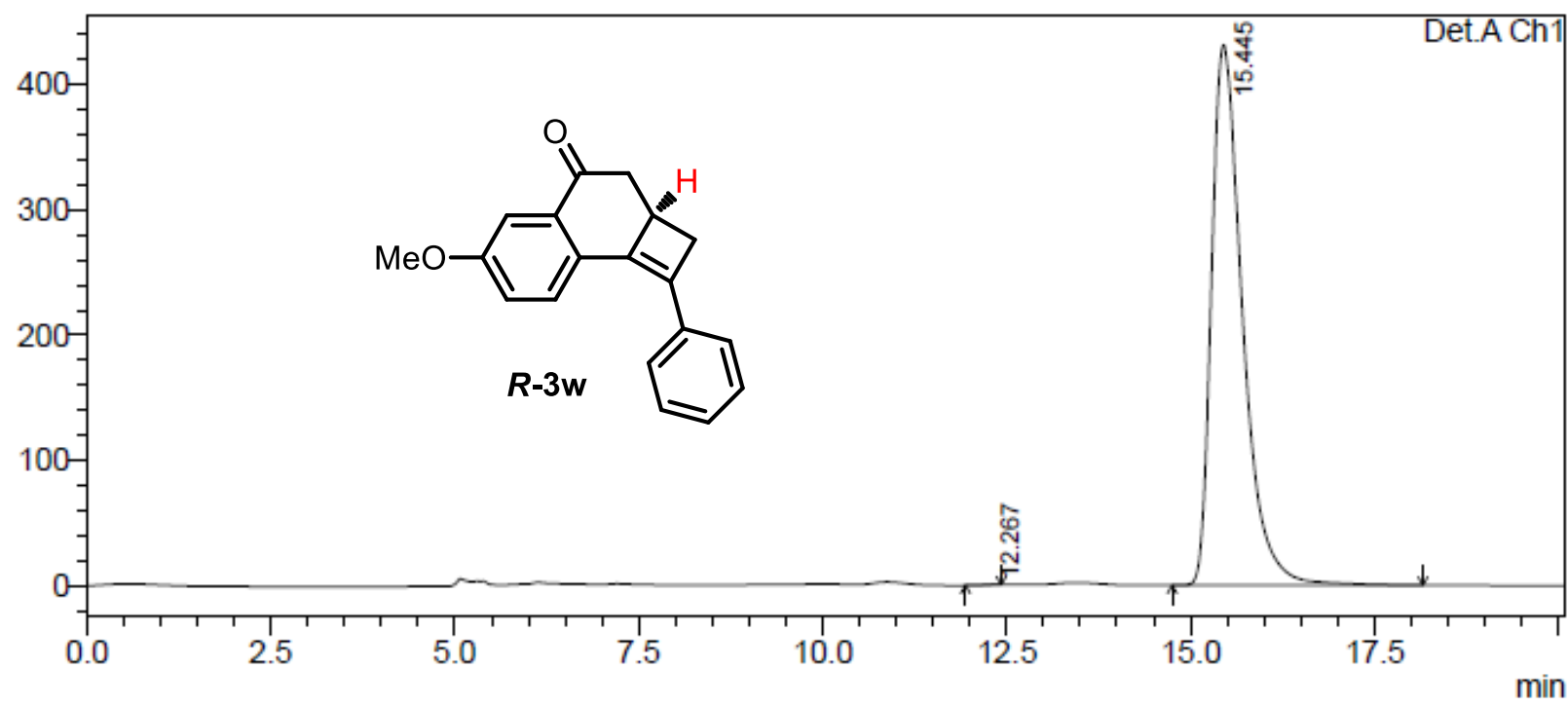

\begin{tabular}{|r|r|r|r|r|r|}
\hline \multicolumn{1}{|c|}{ Peak\# } & \multicolumn{1}{|c|}{ Ret. Time } & \multicolumn{1}{|c|}{ Area } & \multicolumn{1}{|c|}{ Height } & \multicolumn{1}{c|}{ Area \% } & \multicolumn{1}{c|}{ Height \% } \\
\hline 1 & 12.267 & 2882 & 155 & 0.023 & 0.036 \\
\hline 2 & 15.445 & 12651652 & 431285 & 99.977 & 99.964 \\
\hline Total & & 12654533 & 431439 & 100.000 & 100.000 \\
\hline
\end{tabular}




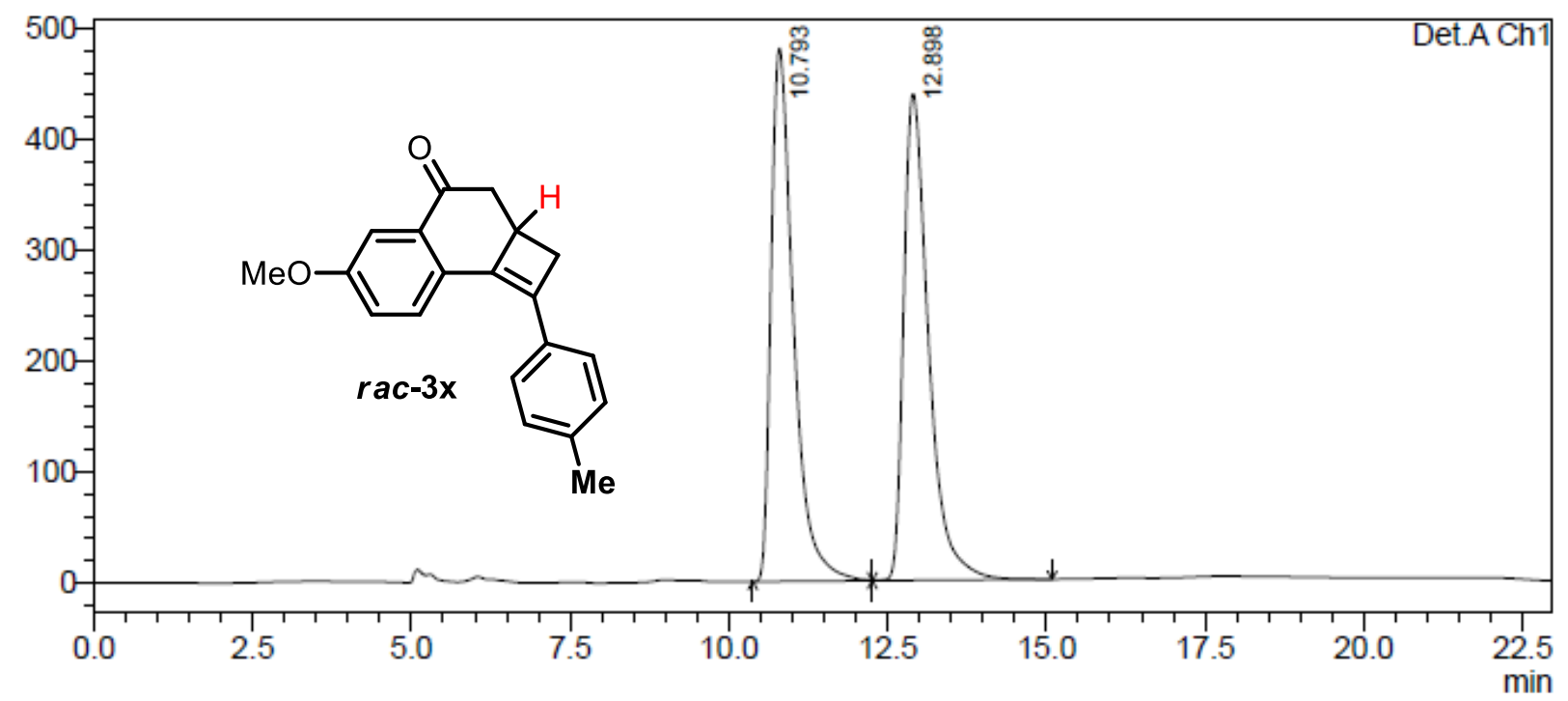

\begin{tabular}{|r|r|r|r|r|r|}
\hline \multicolumn{1}{|c|}{ Peak\# } & Ret. Time & \multicolumn{1}{|c|}{ Area } & Height & \multicolumn{1}{c|}{ Area \% } & \multicolumn{1}{c|}{ Height \% } \\
\hline 1 & 10.793 & 11846308 & 481041 & 49.757 & 52.280 \\
\hline 2 & 12.898 & 11962027 & 439077 & 50.243 & 47.720 \\
\hline Total & & 23808336 & 920118 & 100.000 & 100.000 \\
\hline
\end{tabular}

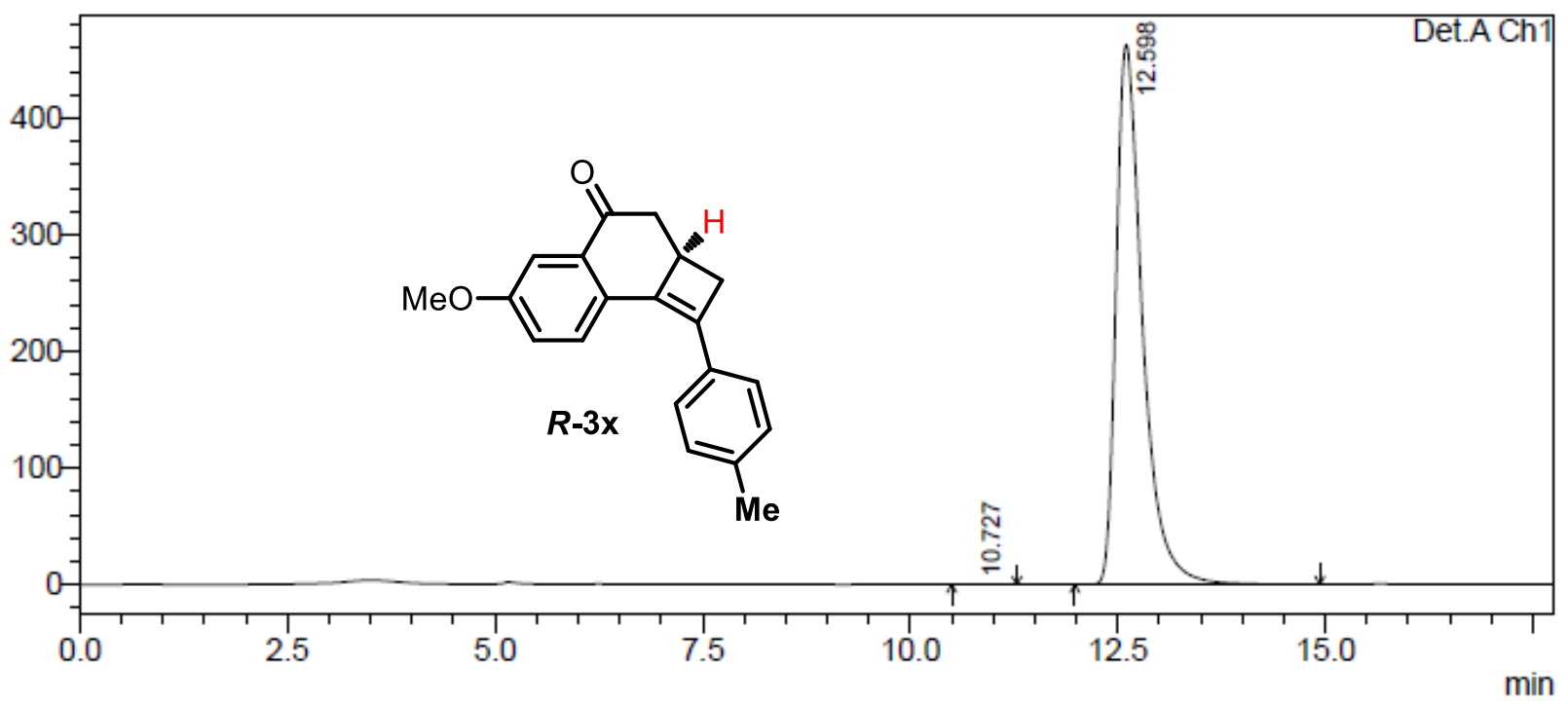

\begin{tabular}{|r|r|r|r|r|r|}
\hline \multicolumn{1}{|c|}{ Peak\# } & \multicolumn{1}{|c|}{ Ret. Time } & \multicolumn{1}{|c|}{ Area } & \multicolumn{1}{c|}{ Height } & \multicolumn{1}{c|}{ Area \% } & \multicolumn{1}{c|}{ Height \% } \\
\hline 1 & 10.727 & 5779 & 275 & 0.056 & 0.059 \\
\hline 2 & 12.598 & 10280186 & 462814 & 99.944 & 99.941 \\
\hline Total & & 10285965 & 463089 & 100.000 & 100.000 \\
\hline
\end{tabular}




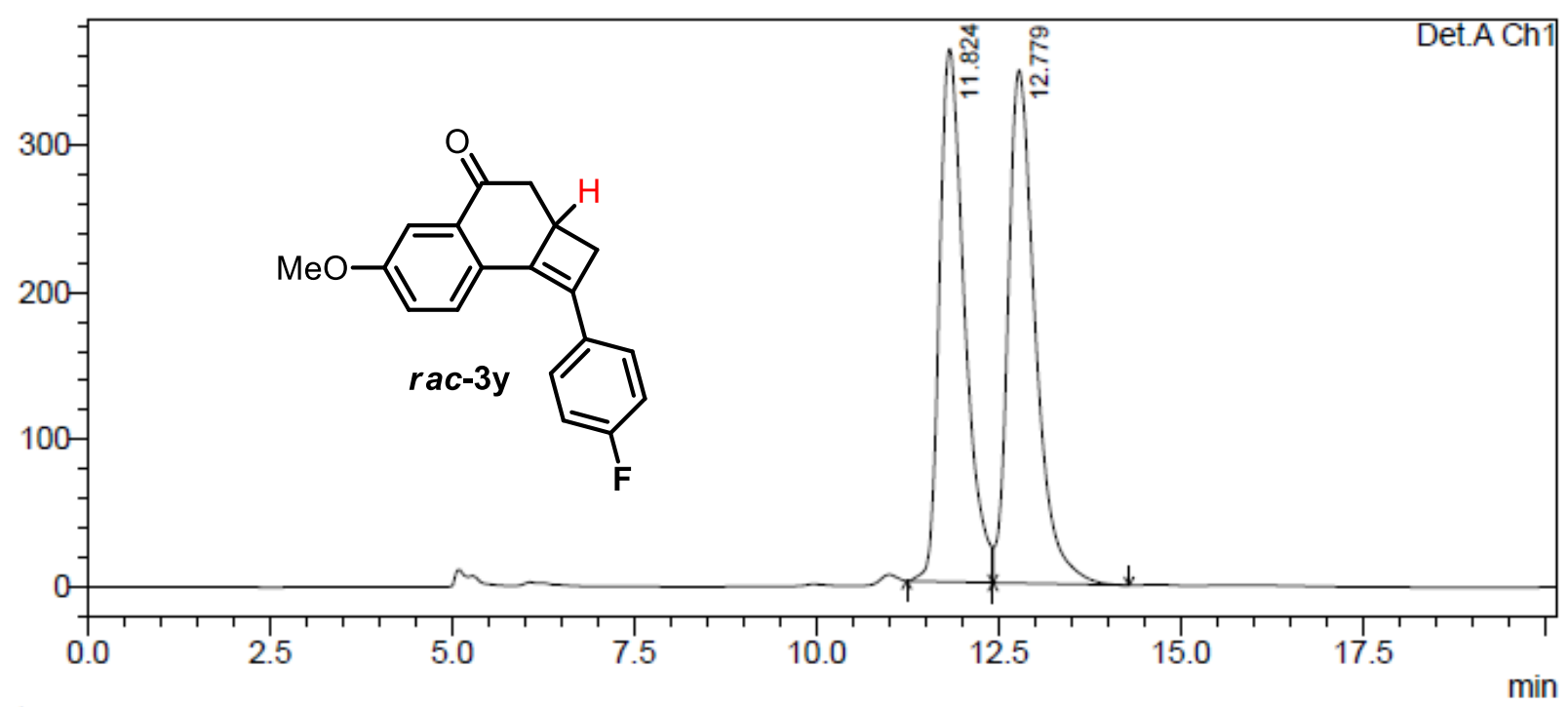

\begin{tabular}{|r|r|r|r|r|r|}
\hline \multicolumn{1}{|c|}{ Peak\# } & Ret. Time & \multicolumn{1}{c|}{ Area } & Height & \multicolumn{1}{c|}{ Area \% } & \multicolumn{1}{c|}{ Height \% } \\
\hline 1 & 11.824 & 8708323 & 361666 & 49.010 & 50.945 \\
\hline 2 & 12.779 & 9060202 & 348252 & 50.990 & 49.055 \\
\hline Total & & 17768525 & 709919 & 100.000 & 100.000 \\
\hline
\end{tabular}

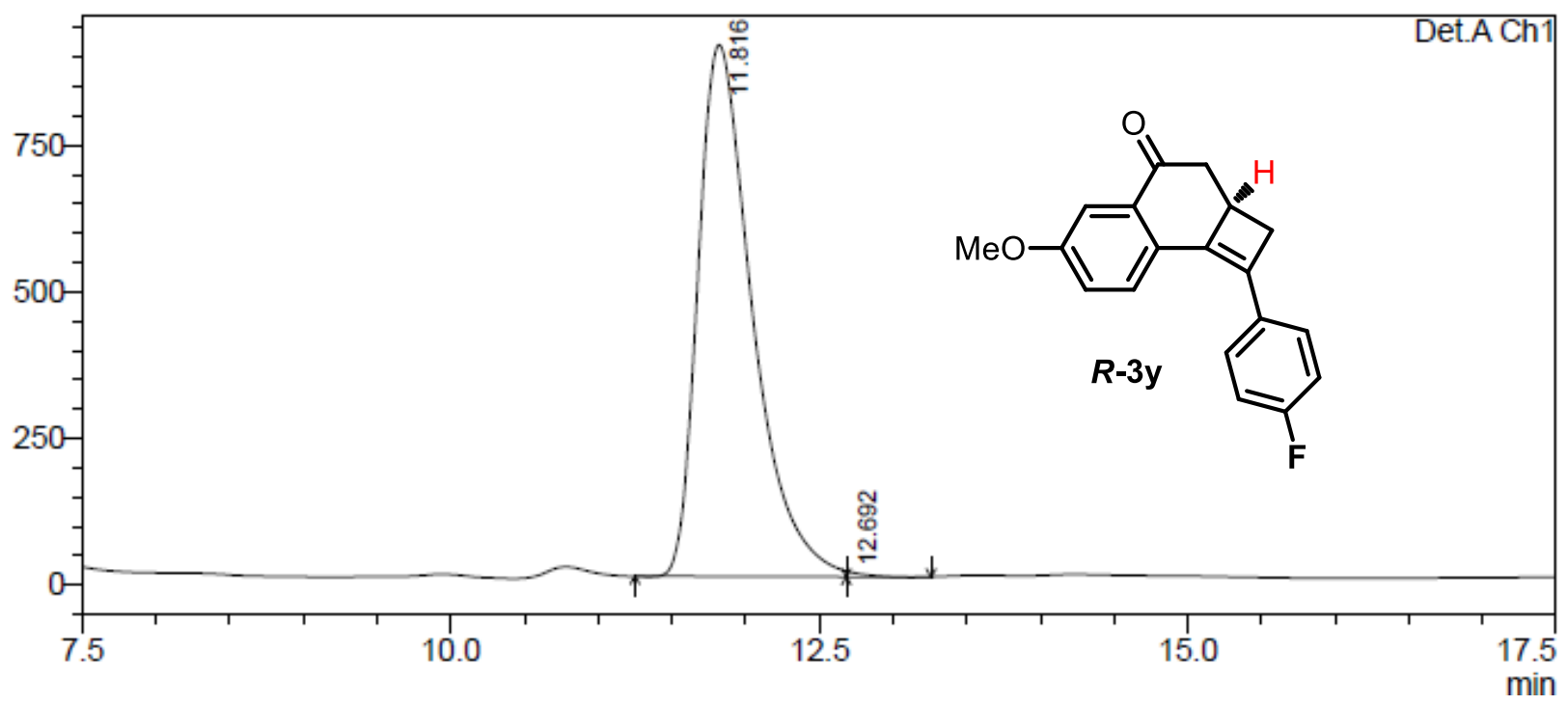

\begin{tabular}{|r|r|r|r|r|r|}
\hline \multicolumn{1}{|c|}{ Peak\# } & \multicolumn{1}{|c|}{ Ret. Time } & \multicolumn{1}{c|}{ Area } & \multicolumn{1}{c|}{ Height } & \multicolumn{1}{c|}{ Area \% } & \multicolumn{1}{c|}{ Height \% } \\
\hline 1 & 11.816 & 23176380 & 906198 & 99.751 & 98.988 \\
\hline 2 & 12.692 & 57933 & 9263 & 0.249 & 1.012 \\
\hline Total & & 23234314 & 915461 & 100.000 & 100.000 \\
\hline
\end{tabular}




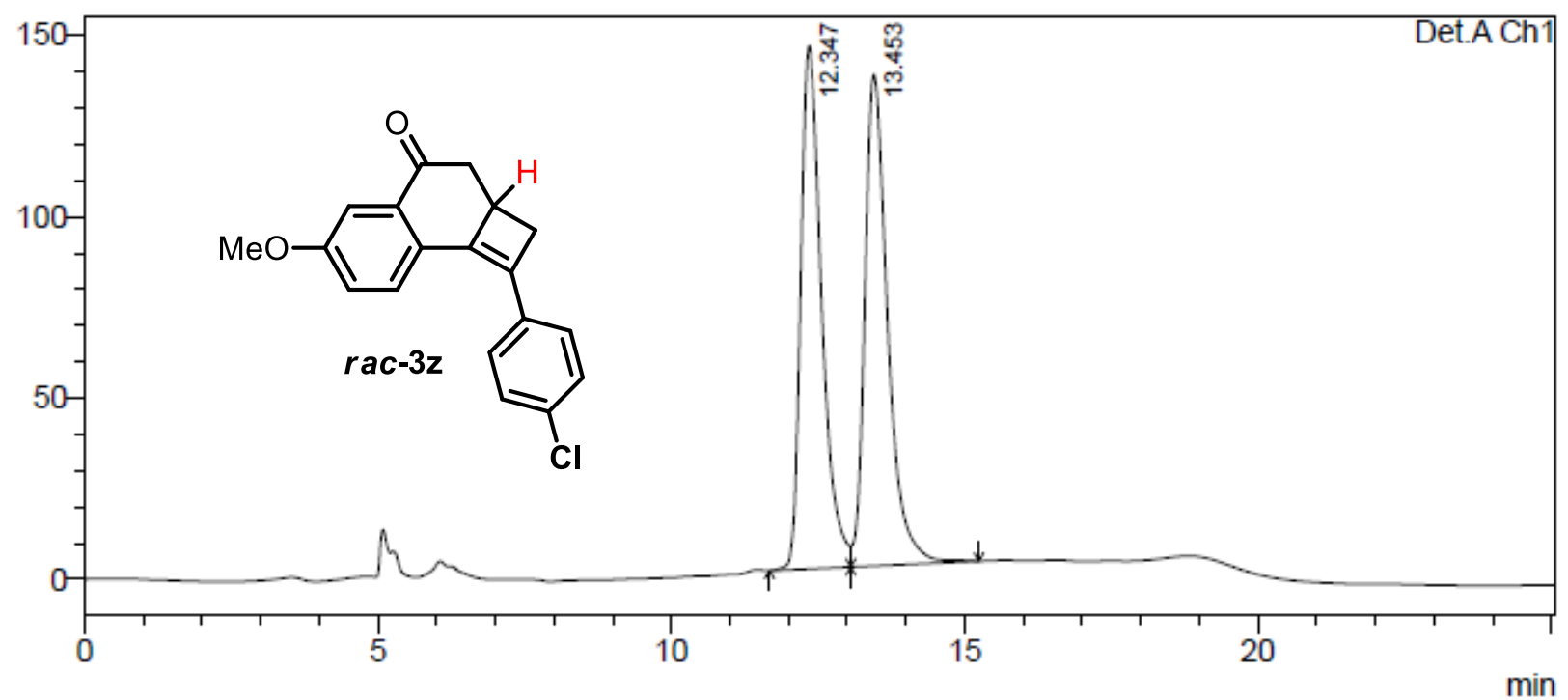

\begin{tabular}{|r|r|r|r|r|r|}
\hline \multicolumn{1}{|c|}{ Peak\# } & Ret. Time & \multicolumn{1}{c|}{ Area } & Height & \multicolumn{1}{|c|}{ Area \% } & \multicolumn{1}{c|}{ Height \% } \\
\hline 1 & 12.347 & 3584112 & 144231 & 49.403 & 51.567 \\
\hline 2 & 13.453 & 3670675 & 135465 & 50.597 & 48.433 \\
\hline Total & & 7254787 & 279696 & 100.000 & 100.000 \\
\hline
\end{tabular}

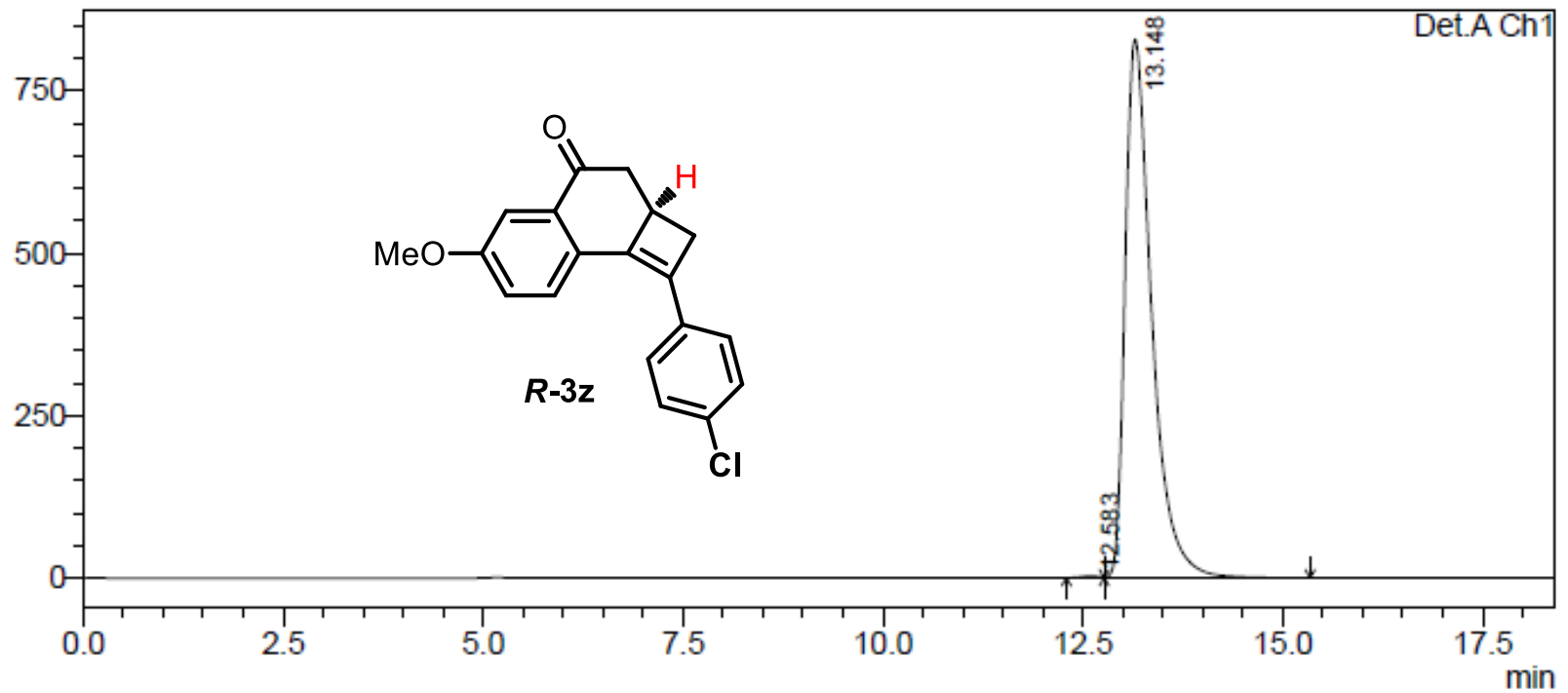

\begin{tabular}{|r|r|r|r|r|r|}
\hline \multicolumn{1}{|c|}{ Peak\# } & Ret. Time & \multicolumn{1}{|c|}{ Area } & \multicolumn{1}{c|}{ Height } & \multicolumn{1}{c|}{ Area \% } & \multicolumn{1}{c|}{ Height \% } \\
\hline 1 & 12.583 & 40252 & 2531 & 0.210 & 0.305 \\
\hline 2 & 13.148 & 19108550 & 828174 & 99.790 & 99.695 \\
\hline Total & & 19148803 & 830705 & 100.000 & 100.000 \\
\hline
\end{tabular}




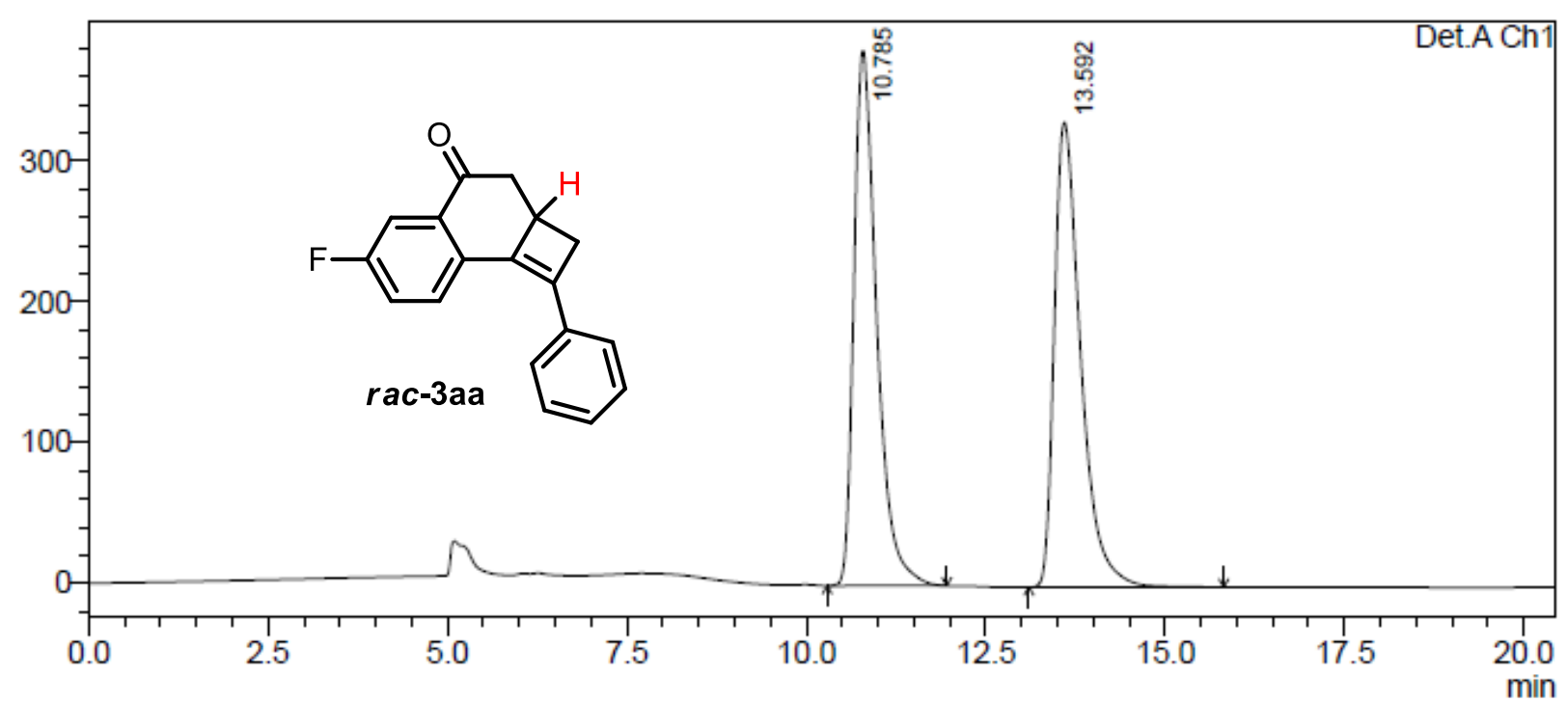

\begin{tabular}{|r|r|r|r|r|r|}
\hline \multicolumn{1}{|c|}{ Peak\# } & Ret. Time & \multicolumn{1}{c|}{ Area } & Height & \multicolumn{1}{c|}{ Area \% } & \multicolumn{1}{c|}{ Height \% } \\
\hline 1 & 10.785 & 8425380 & 380304 & 49.968 & 53.535 \\
\hline 2 & 13.592 & 8436075 & 330075 & 50.032 & 46.465 \\
\hline Total & & 16861455 & 710379 & 100.000 & 100.000 \\
\hline
\end{tabular}

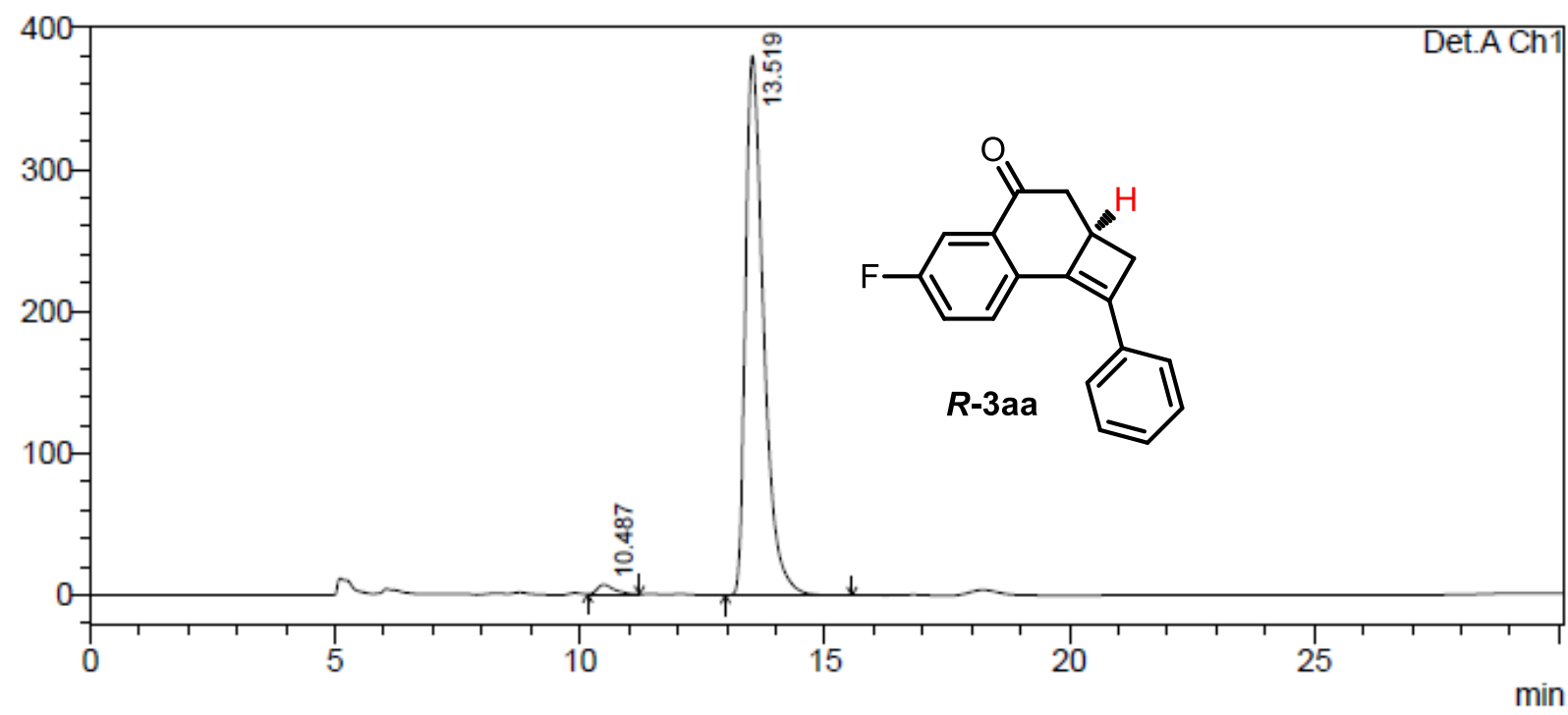

\begin{tabular}{|r|r|r|r|r|r|}
\hline \multicolumn{1}{|c|}{ Peak\# } & Ret. Time & \multicolumn{1}{c|}{ Area } & \multicolumn{1}{c|}{ Height } & \multicolumn{1}{c|}{ Area \% } & \multicolumn{1}{c|}{ Height \% } \\
\hline 1 & 10.487 & 162129 & 6839 & 1.625 & 1.768 \\
\hline 2 & 13.519 & 9814496 & 380013 & 98.375 & 98.232 \\
\hline Total & & 9976625 & 386852 & 100.000 & 100.000 \\
\hline
\end{tabular}




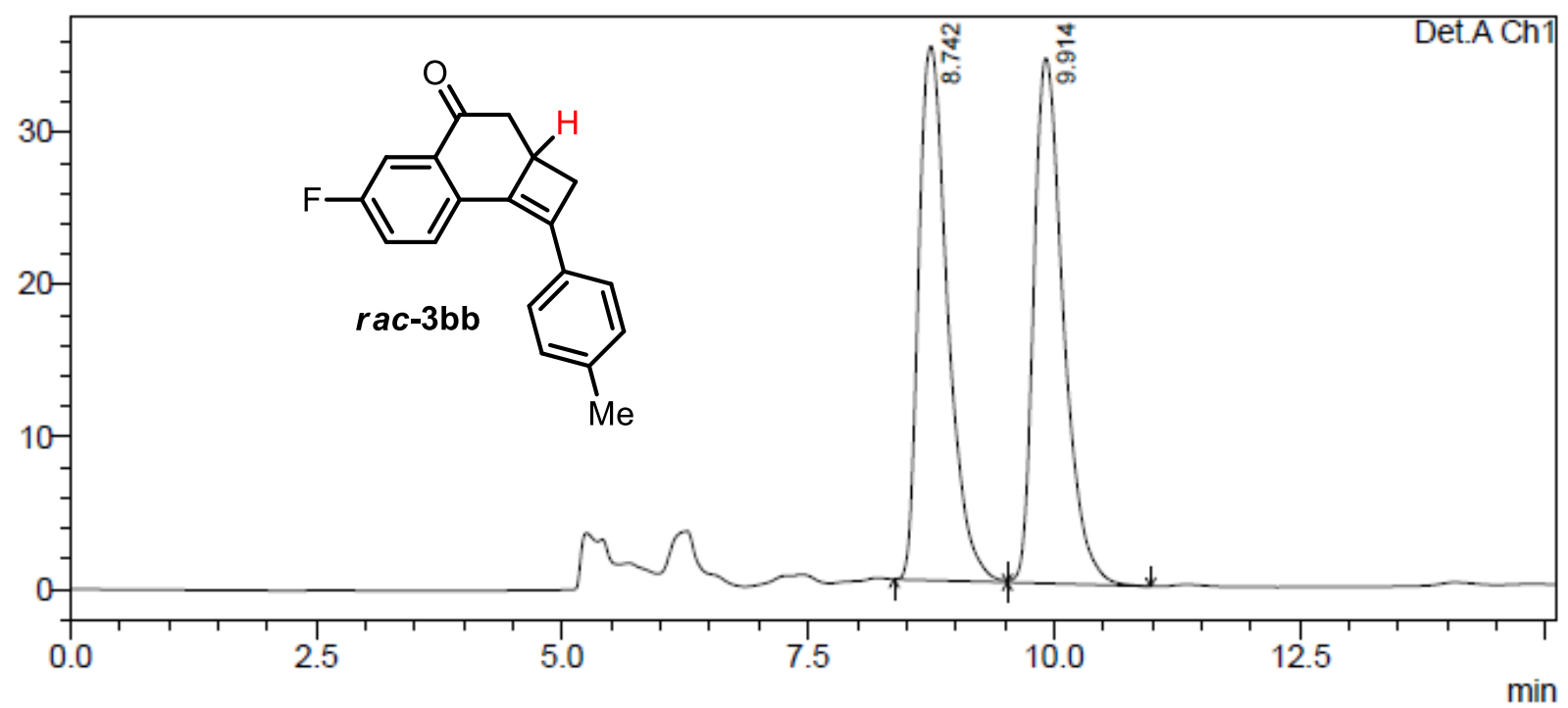

\begin{tabular}{|r|r|r|r|r|r|}
\hline \multicolumn{1}{|c|}{ Peak\# } & \multicolumn{1}{|c|}{ Ret. Time } & \multicolumn{1}{c|}{ Area } & Height & \multicolumn{1}{c|}{ Area \% } & \multicolumn{1}{c|}{ Height \% } \\
\hline 1 & 8.742 & 722657 & 35107 & 49.898 & 50.420 \\
\hline 2 & 9.914 & 725598 & 34523 & 50.102 & 49.580 \\
\hline Total & & 1448255 & 69630 & 100.000 & 100.000 \\
\hline
\end{tabular}

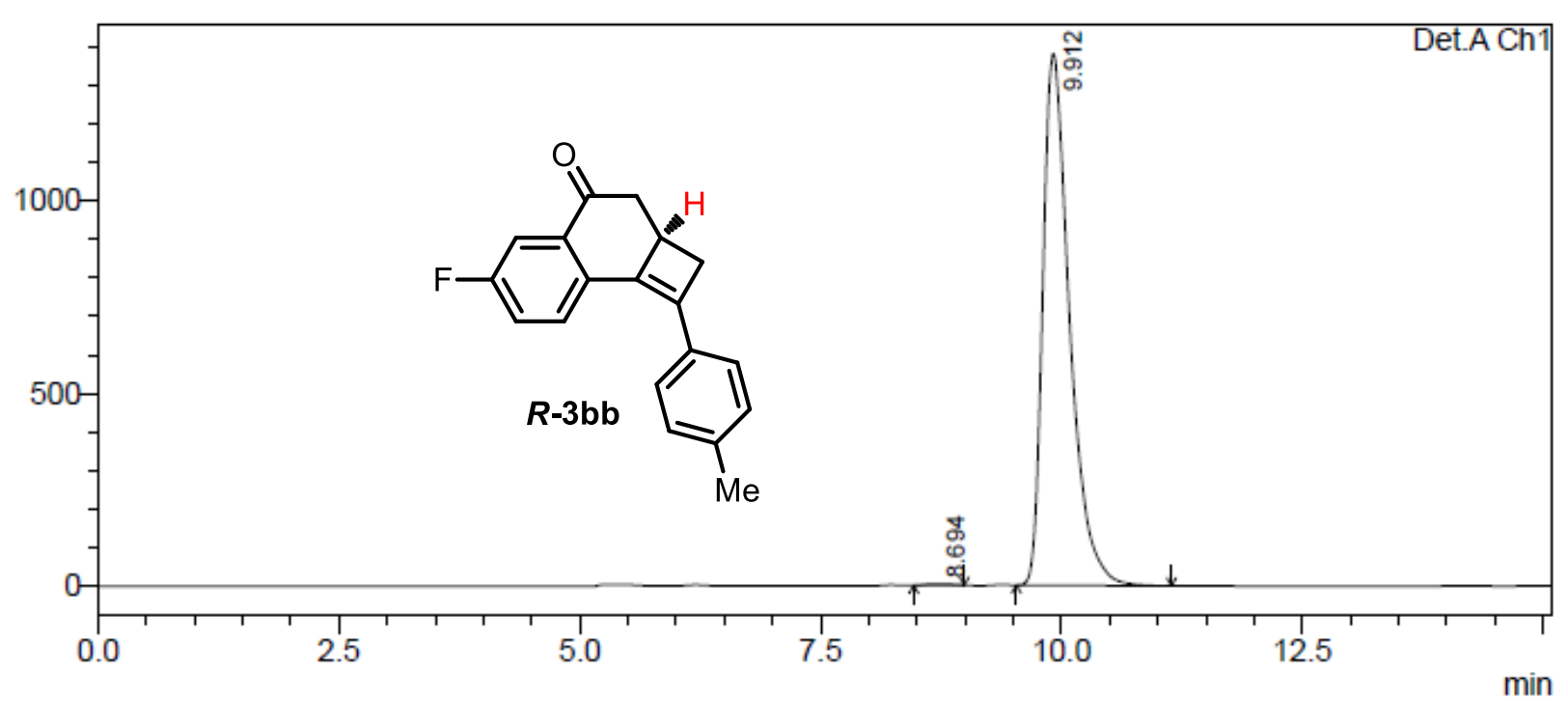

\begin{tabular}{|r|r|r|r|r|r|}
\hline \multicolumn{1}{|c|}{ Peak\# } & \multicolumn{1}{|c|}{ Ret. Time } & \multicolumn{1}{c|}{ Area } & Height & \multicolumn{1}{c|}{ Area \% } & \multicolumn{1}{c|}{ Height \% } \\
\hline 1 & 8.694 & 62915 & 3891 & 0.235 & 0.281 \\
\hline 2 & 9.912 & 26680589 & 1379996 & 99.765 & 99.719 \\
\hline Total & & 26743504 & 1383886 & 100.000 & 100.000 \\
\hline
\end{tabular}




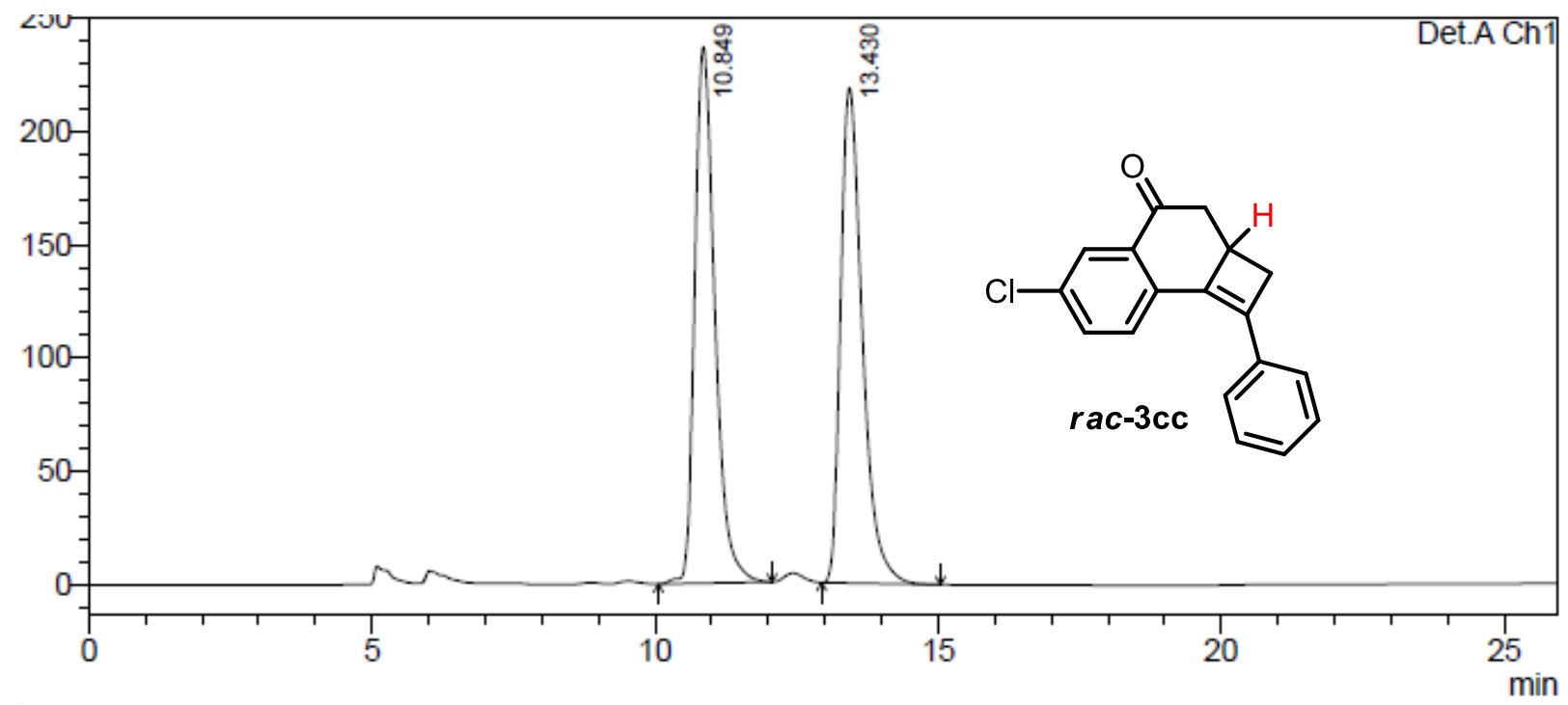

\begin{tabular}{|r|r|r|r|r|r|}
\hline \multicolumn{1}{|c|}{ Peak\# } & Ret. Time & \multicolumn{1}{c|}{ Area } & \multicolumn{1}{c|}{ Height } & \multicolumn{1}{c|}{ Area \% } & \multicolumn{1}{c|}{ Height \% } \\
\hline 1 & 10.849 & 5856856 & 236379 & 50.099 & 51.969 \\
\hline 2 & 13.430 & 5833733 & 218471 & 49.901 & 48.031 \\
\hline Total & & 11690589 & 454850 & 100.000 & 100.000 \\
\hline
\end{tabular}

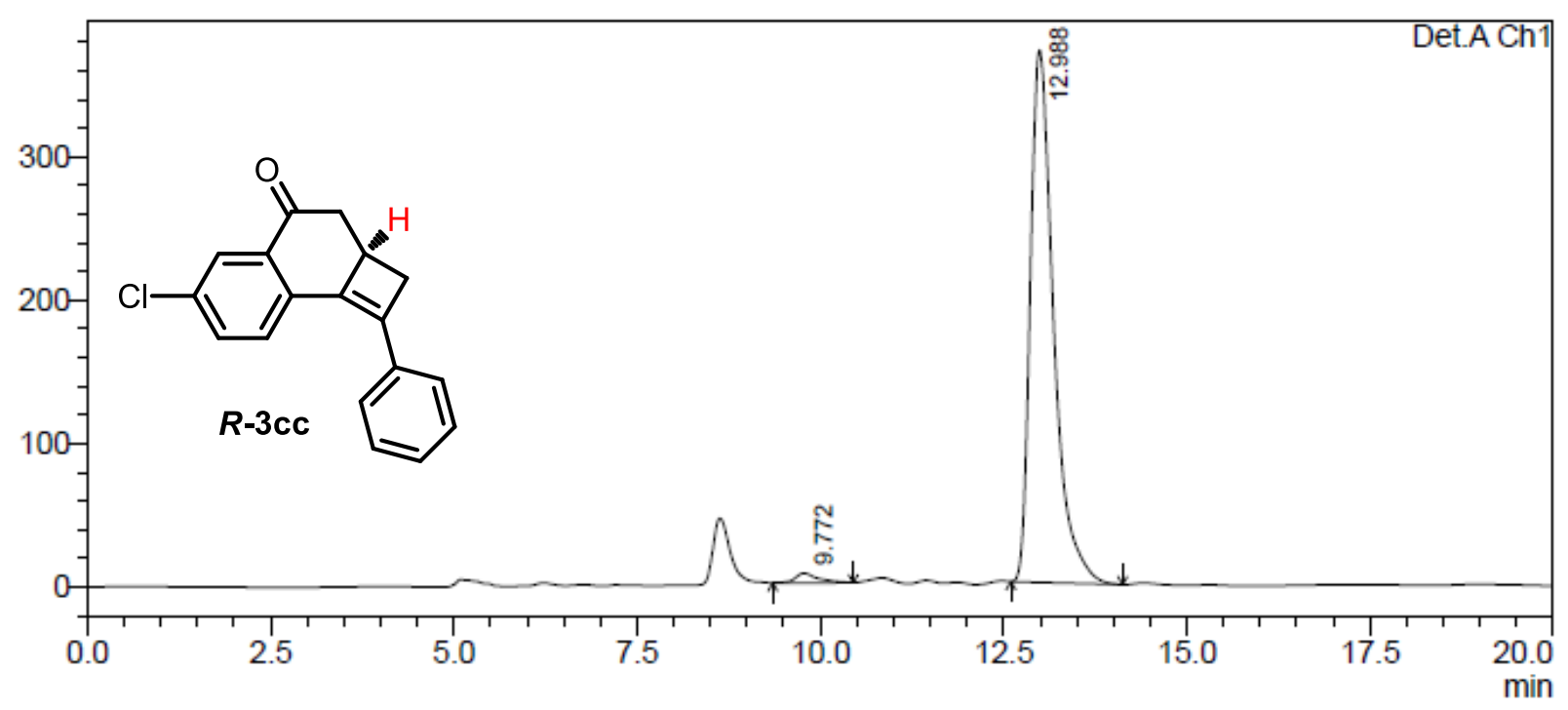

\begin{tabular}{|r|r|r|r|r|r|}
\hline \multicolumn{1}{|c|}{ Peak\# } & \multicolumn{1}{|c|}{ Ret. Time } & \multicolumn{1}{c|}{ Area } & \multicolumn{1}{c|}{ Height } & \multicolumn{1}{c|}{ Area \% } & \multicolumn{1}{c|}{ Height \% } \\
\hline 1 & 9.772 & 148853 & 6753 & 1.783 & 1.786 \\
\hline 2 & 12.988 & 8199159 & 371440 & 98.217 & 98.214 \\
\hline Total & & 8348012 & 378193 & 100.000 & 100.000 \\
\hline
\end{tabular}




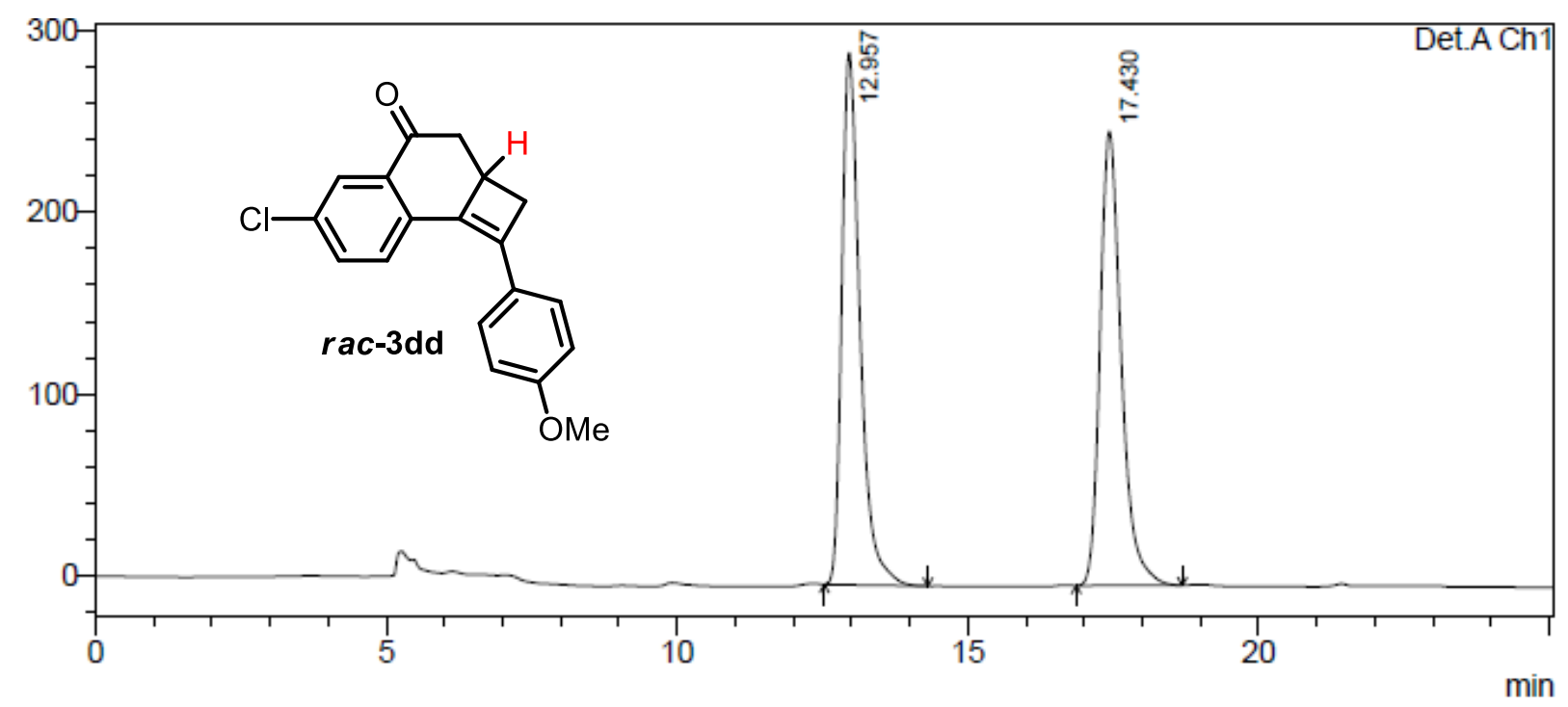

\begin{tabular}{|r|r|r|r|r|r|}
\hline \multicolumn{1}{|c|}{ Peak\# } & Ret. Time & \multicolumn{1}{c|}{ Area } & Height & \multicolumn{1}{c|}{ Area \% } & \multicolumn{1}{c|}{ Height \% } \\
\hline 1 & 12.957 & 6356037 & 292026 & 50.103 & 53.955 \\
\hline 2 & 17.430 & 6329849 & 249214 & 49.897 & 46.045 \\
\hline Total & & 12685886 & 541240 & 100.000 & 100.000 \\
\hline
\end{tabular}

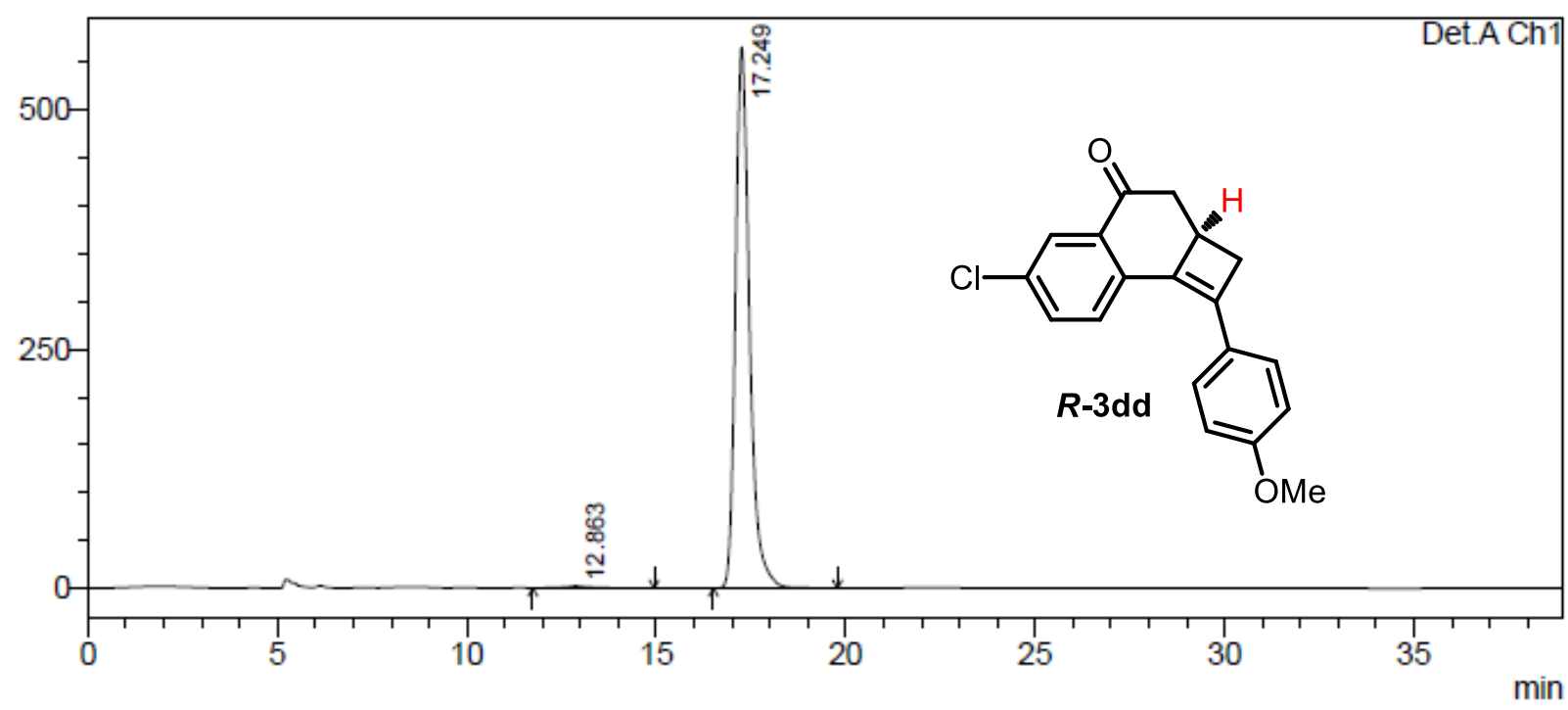

\begin{tabular}{|r|r|r|r|r|r|}
\hline \hline Peak\# & Ret. Time & \multicolumn{1}{c|}{ Area } & \multicolumn{1}{c|}{ Height } & \multicolumn{1}{c|}{ Area \% } & \multicolumn{1}{c|}{ Height \% } \\
\hline 1 & 12.863 & 101034 & 2046 & 0.683 & 0.360 \\
\hline 2 & 17.249 & 14684832 & 565823 & 99.317 & 99.640 \\
\hline Total & & 14785867 & 567869 & 100.000 & 100.000 \\
\hline
\end{tabular}




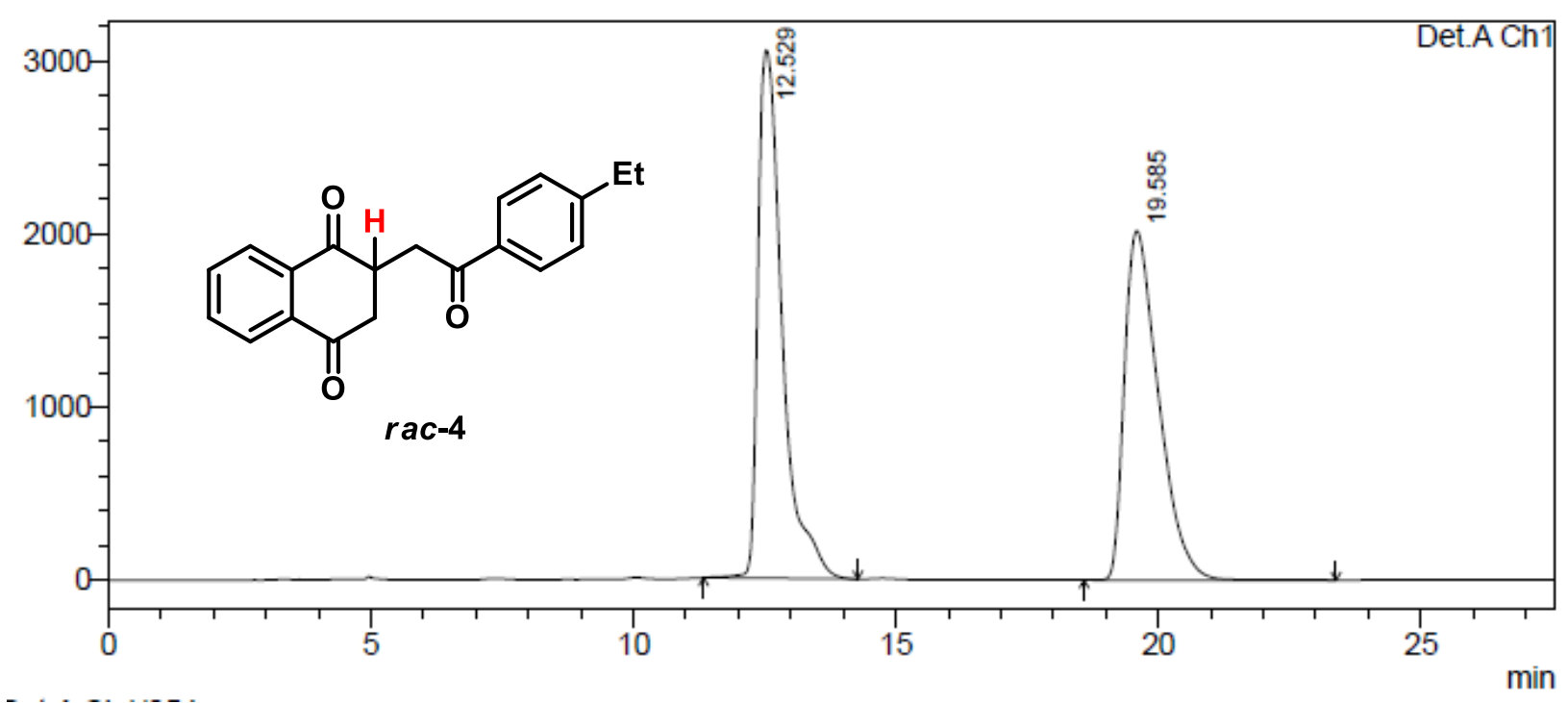

\begin{tabular}{|r|r|r|r|r|r|}
\hline \multicolumn{1}{|c|}{ Peak\# } & \multicolumn{1}{|c|}{ Ret. Time } & \multicolumn{1}{c|}{ Area } & Height & \multicolumn{1}{c|}{ Area \% } & \multicolumn{1}{c|}{ Height \% } \\
\hline 1 & 12.529 & 98420795 & 3049844 & 51.941 & 60.207 \\
\hline 2 & 19.585 & 91065333 & 2015747 & 48.059 & 39.793 \\
\hline Total & & 189486127 & 5065592 & 100.000 & 100.000 \\
\hline
\end{tabular}

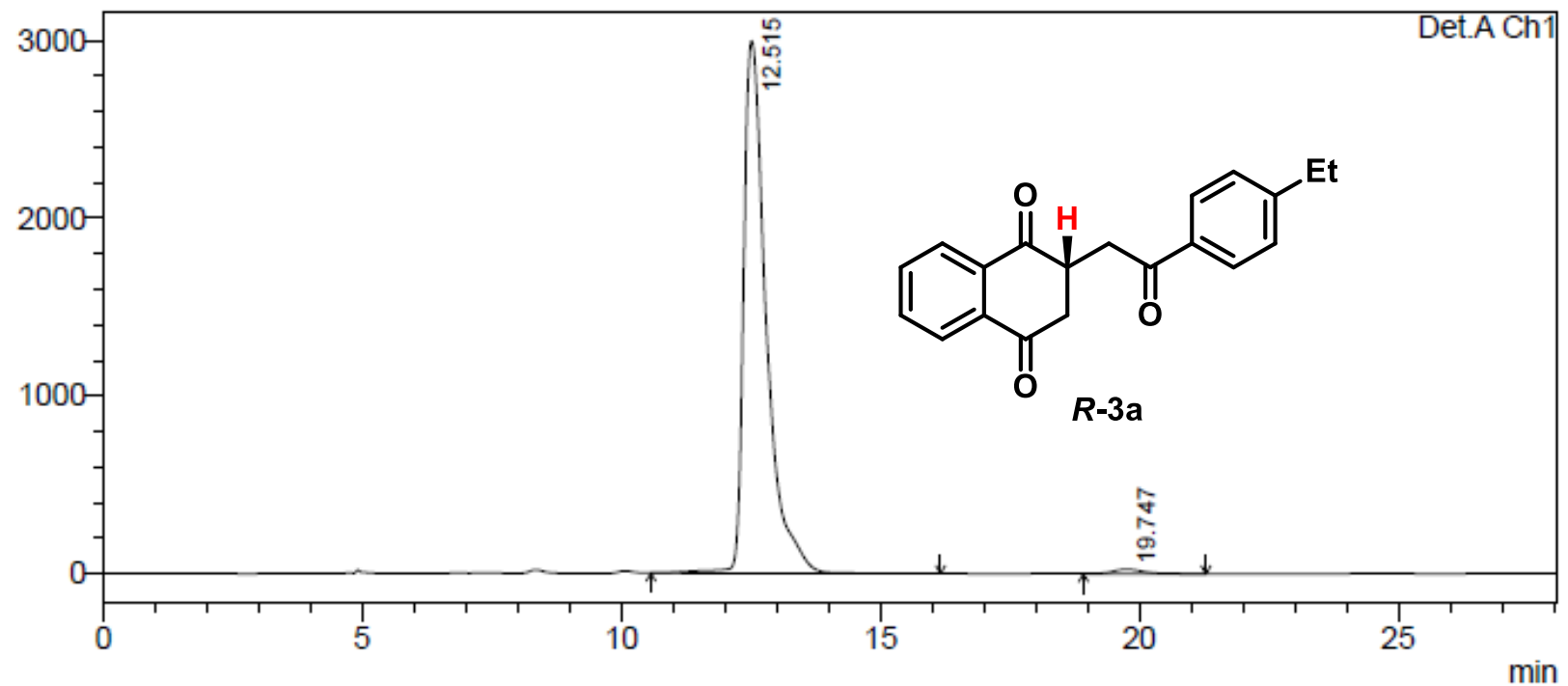

\begin{tabular}{|r|r|r|r|r|r|}
\hline \multicolumn{1}{|c|}{ Peak\# } & \multicolumn{1}{|c|}{ Ret. Time } & \multicolumn{1}{c|}{ Area } & \multicolumn{1}{c|}{ Height } & \multicolumn{1}{c|}{ Area \% } & \multicolumn{1}{c|}{ Height \% } \\
\hline 1 & 12.515 & 91040680 & 2995994 & 98.902 & 99.162 \\
\hline 2 & 19.747 & 1010821 & 25326 & 1.098 & 0.838 \\
\hline Total & & 92051501 & 3021320 & 100.000 & 100.000 \\
\hline
\end{tabular}




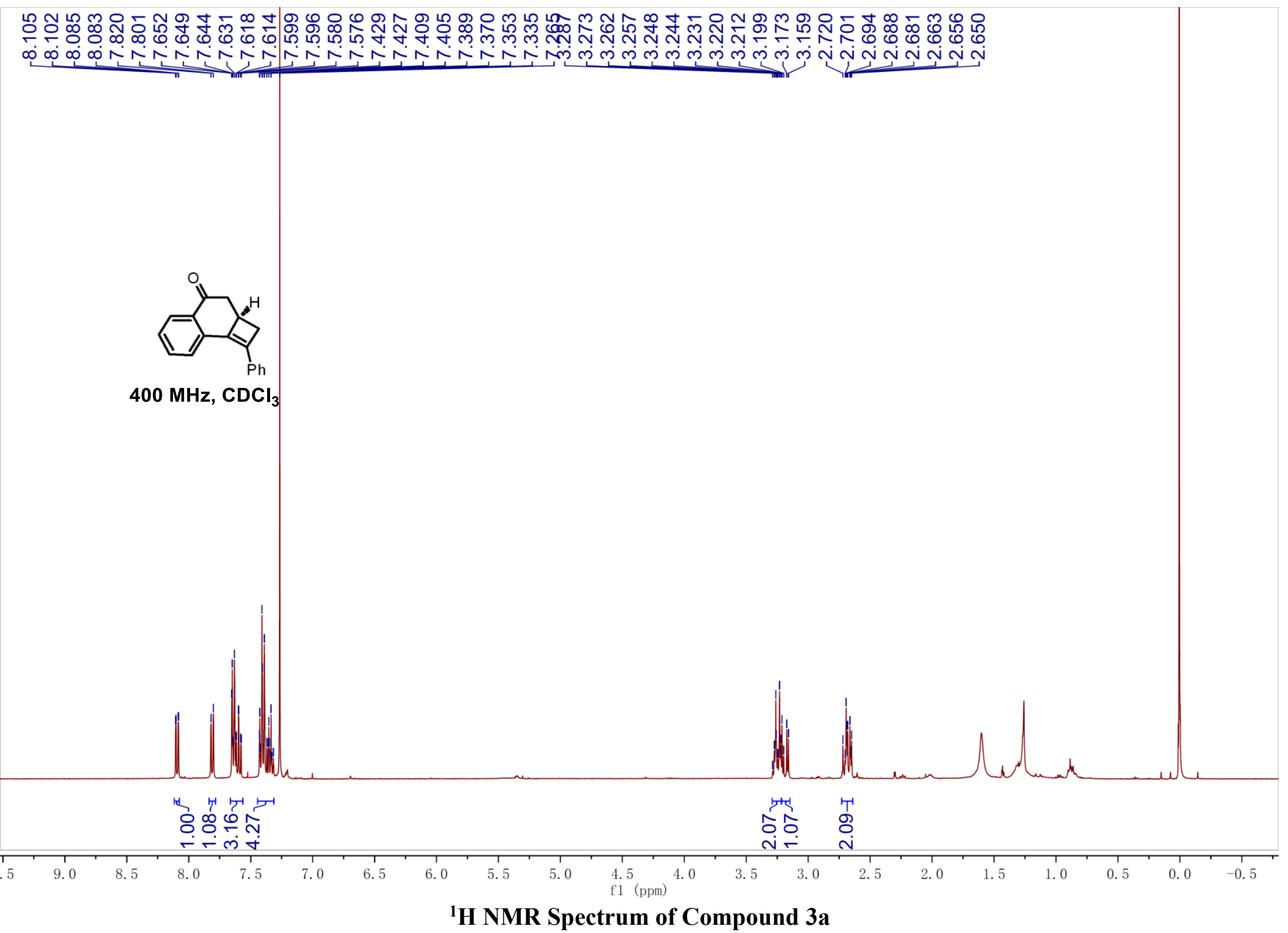




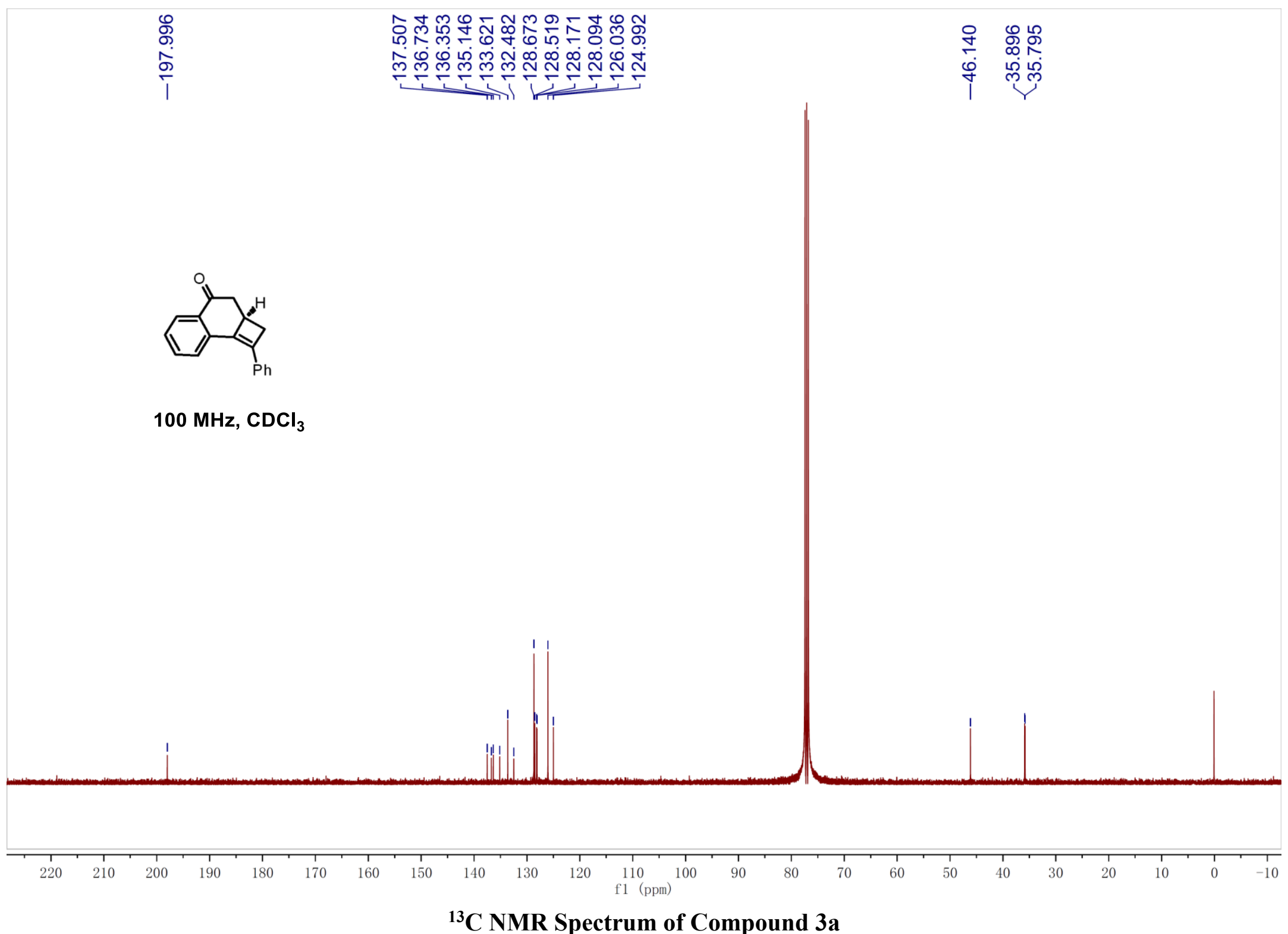



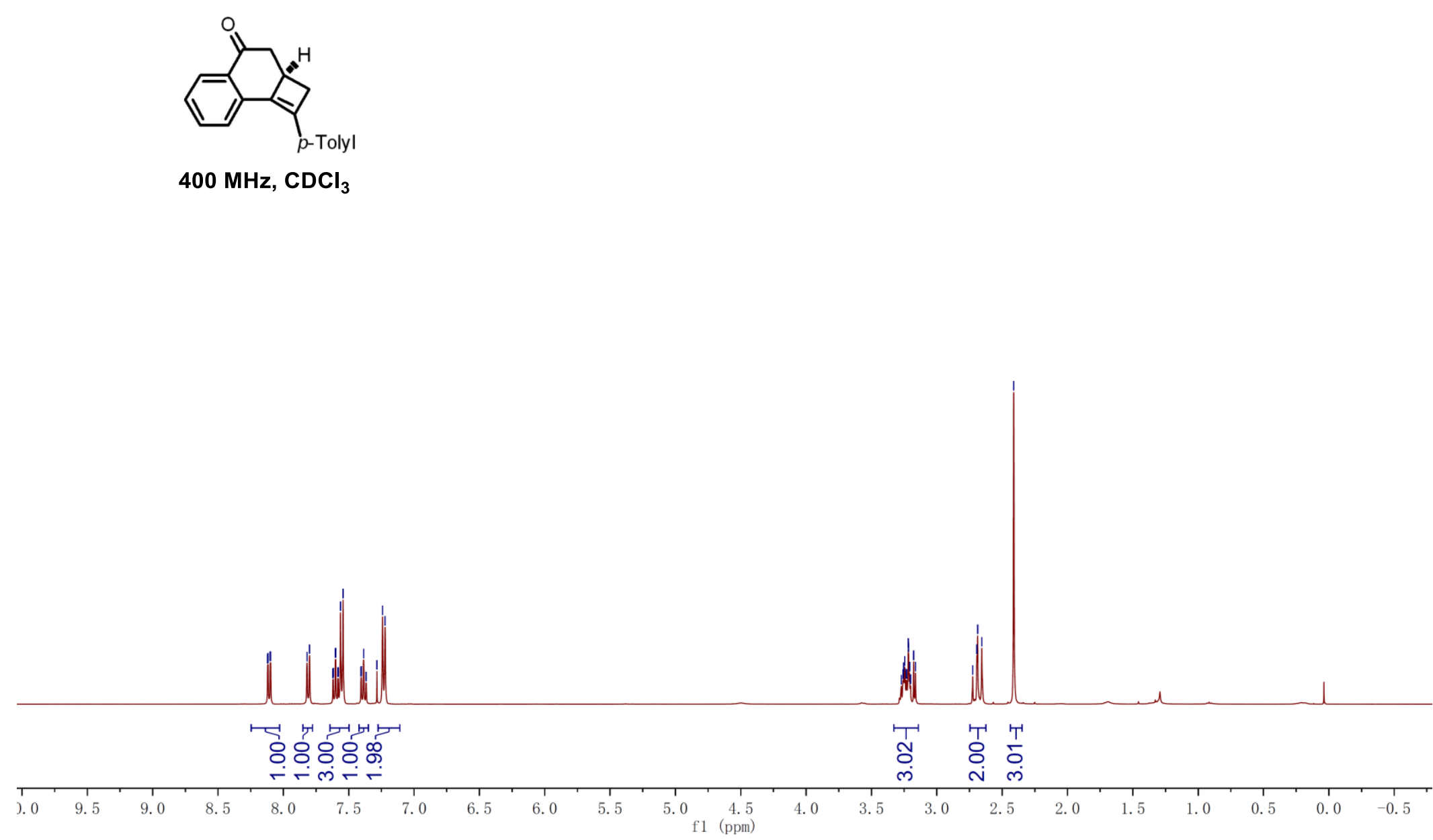

${ }^{1}$ H NMR Spectrum of Compound 3b 


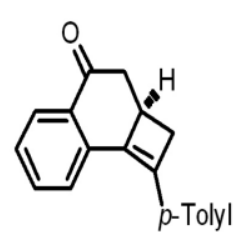

$100 \mathrm{MHz}, \mathrm{CDCl}_{3}$

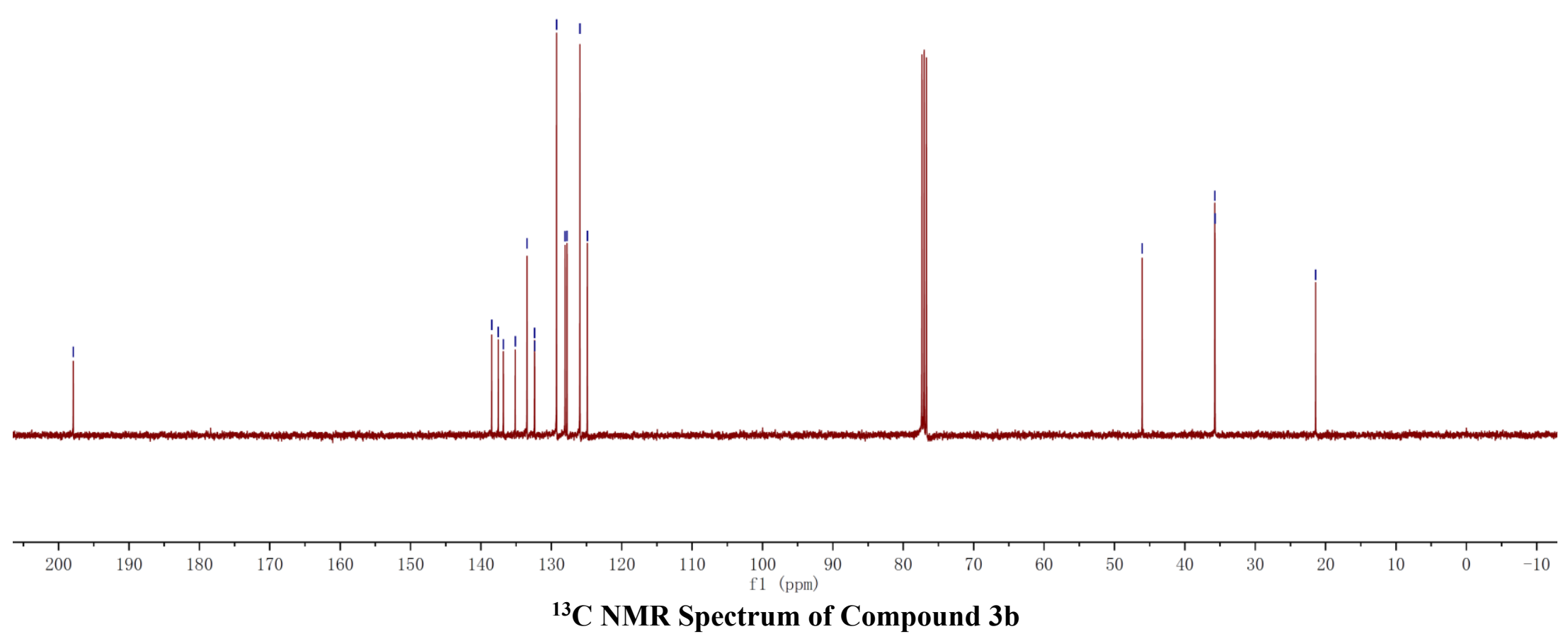




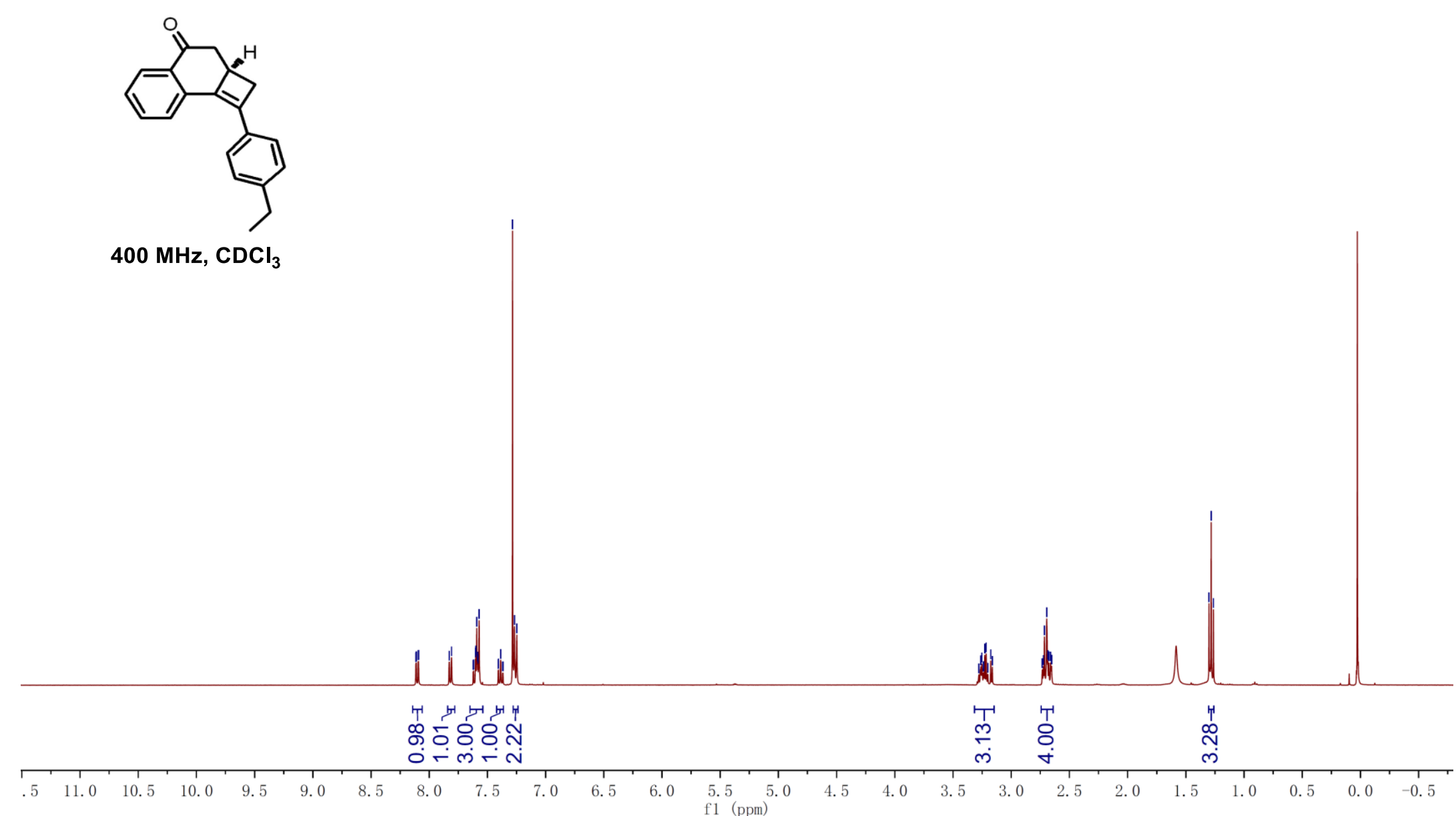

${ }^{1} \mathrm{H}$ NMR Spectrum of Compound 3c 


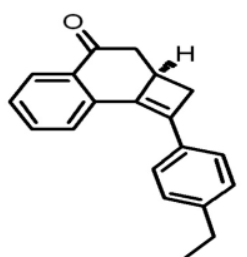

$100 \mathrm{MHz}, \mathrm{CDCl}_{3}$
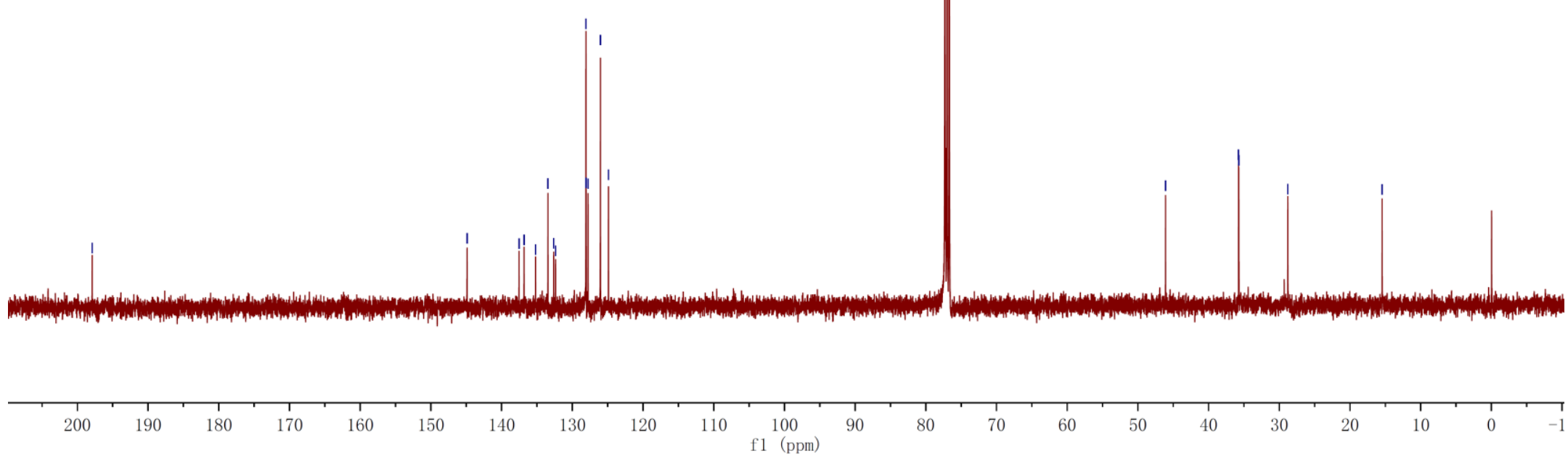

${ }^{13} \mathrm{C}$ NMR Spectrum of Compound $3 \mathrm{c}$ 


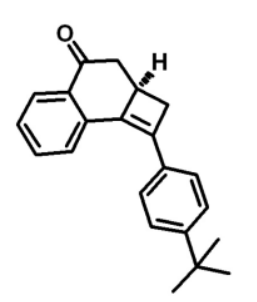

$400 \mathrm{MHz}^{\mathrm{CDCl}_{3}}$

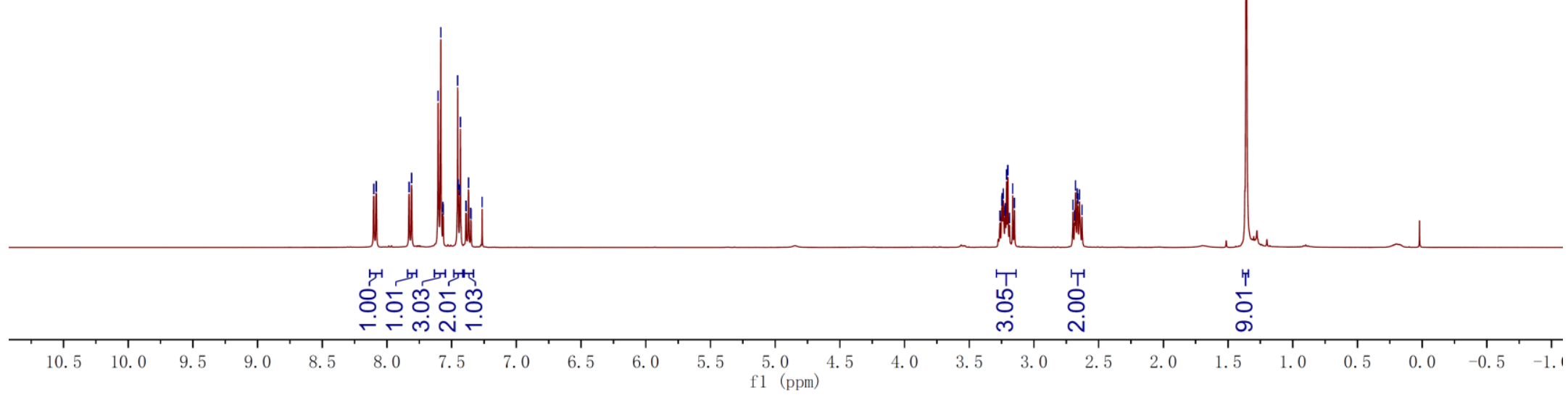

${ }^{1}$ H NMR Spectrum of Compound 3d 


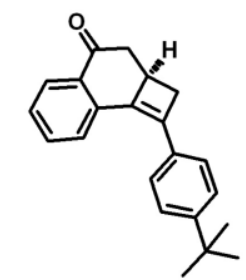

$100 \mathrm{MHz}, \mathrm{CDCl}_{3}$
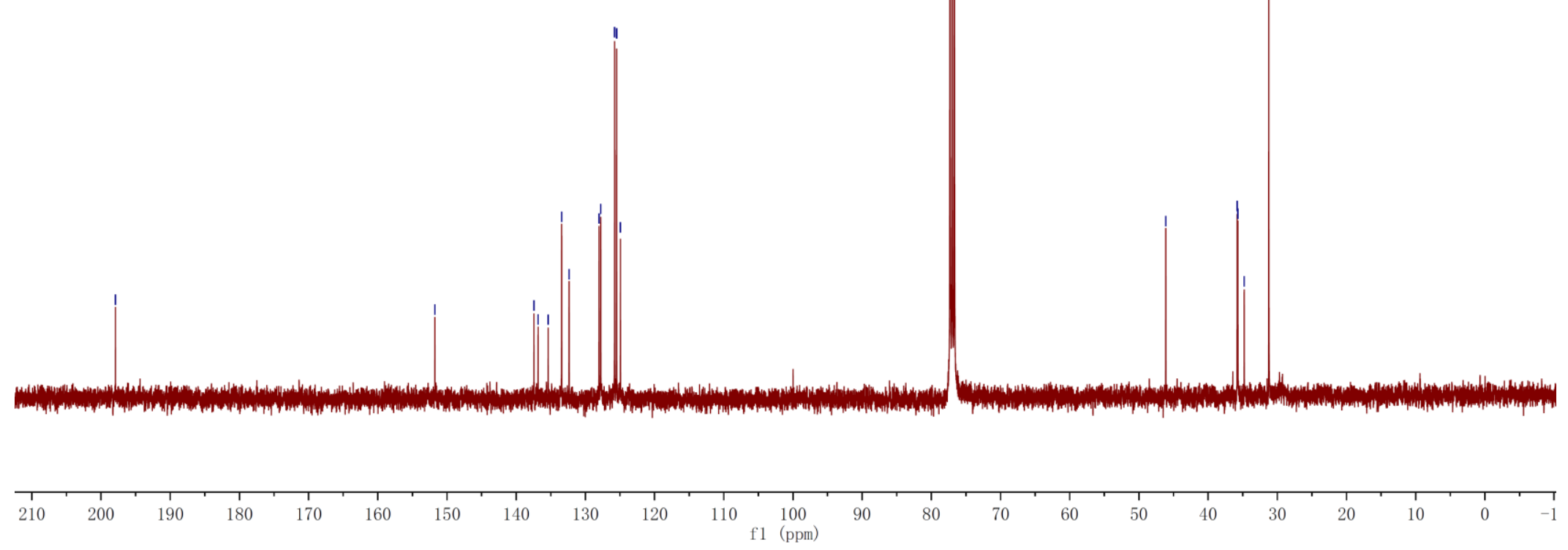

${ }^{13}$ C NMR Spectrum of Compound 3d 


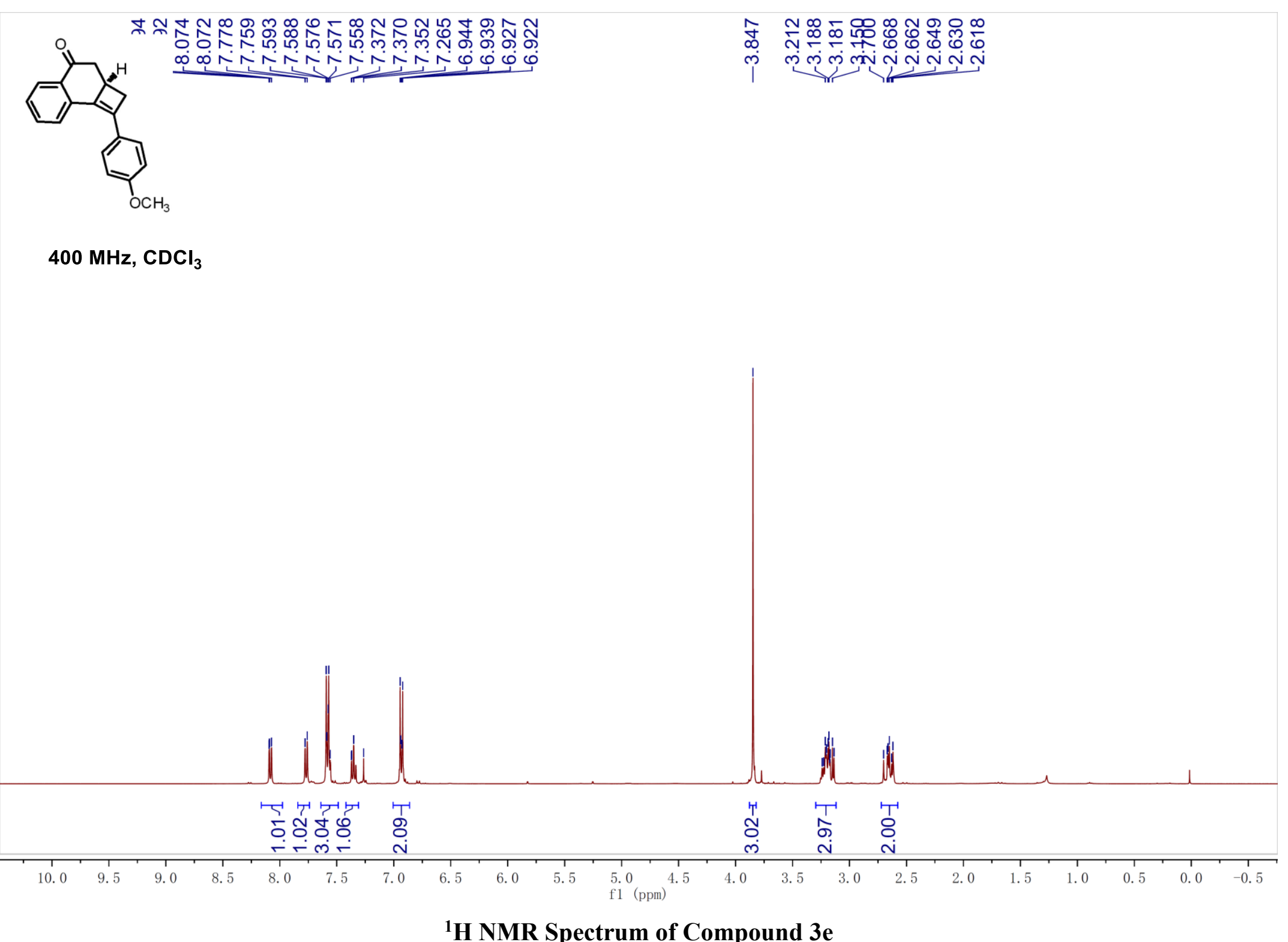




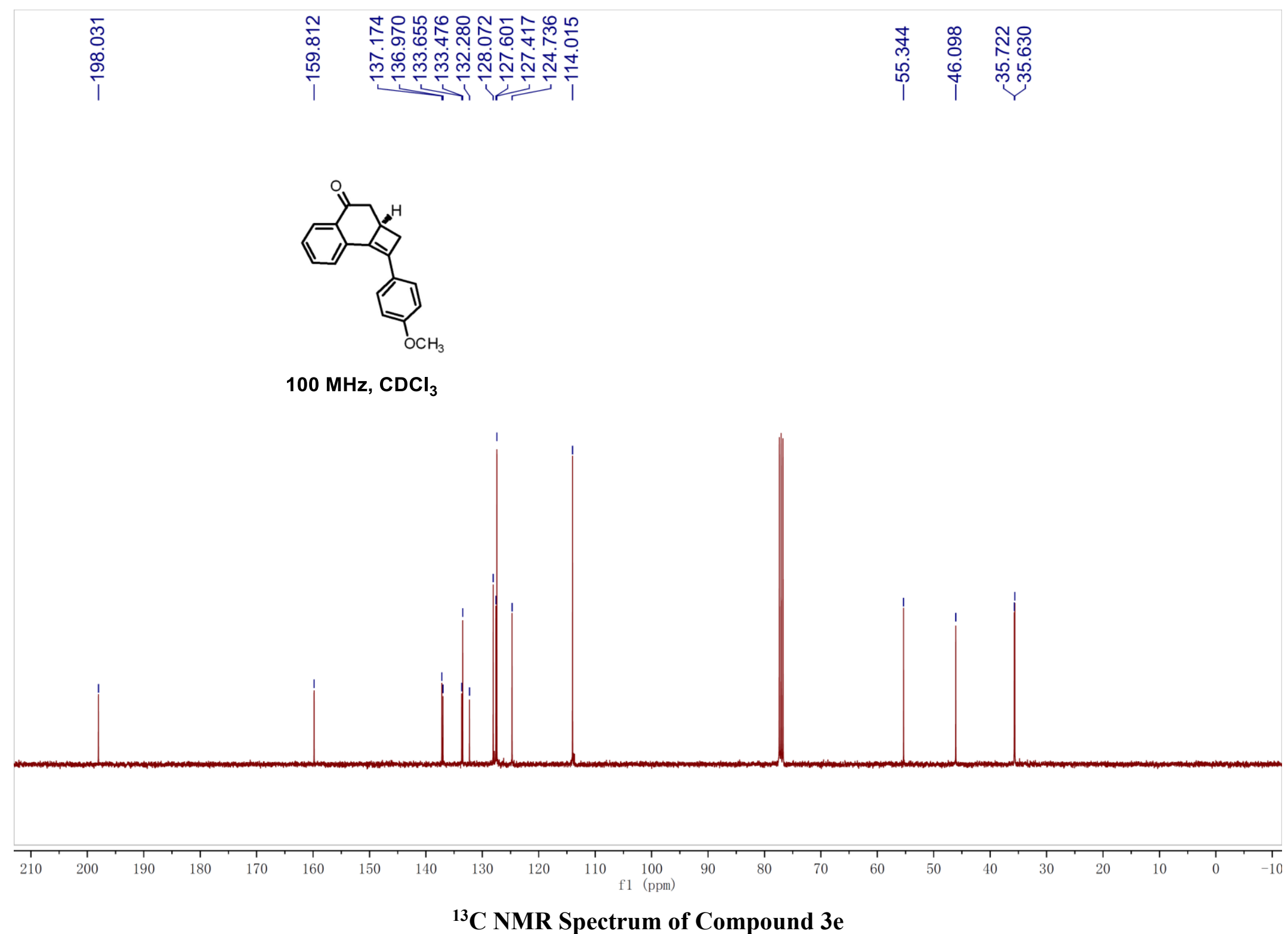




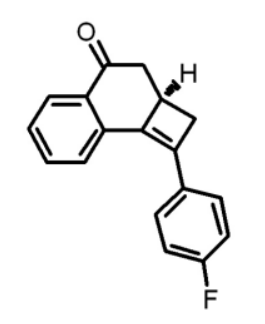

$400 \mathrm{MHz}^{\mathrm{CDCl}_{3}}$

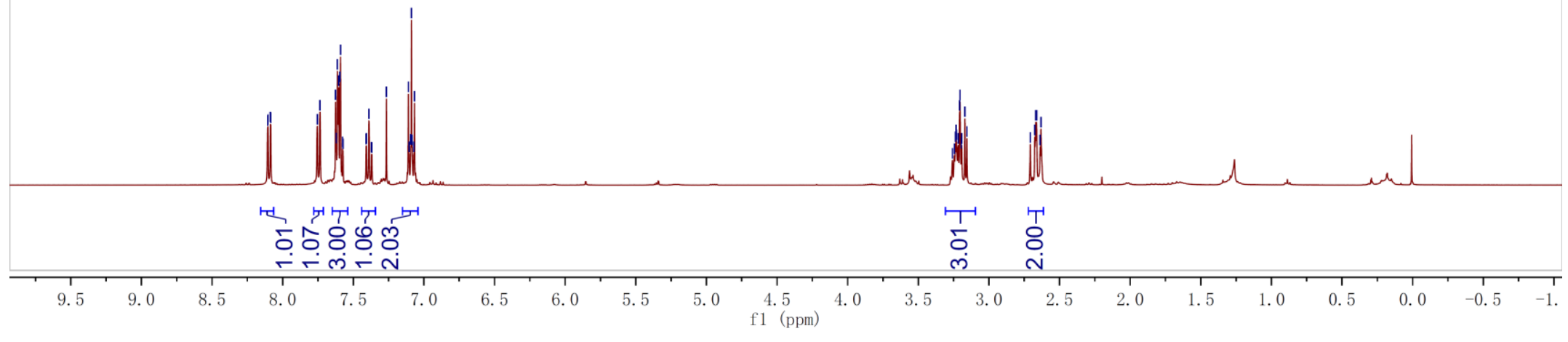

${ }^{1} \mathrm{H}$ NMR Spectrum of Compound $3 \mathrm{f}$ 


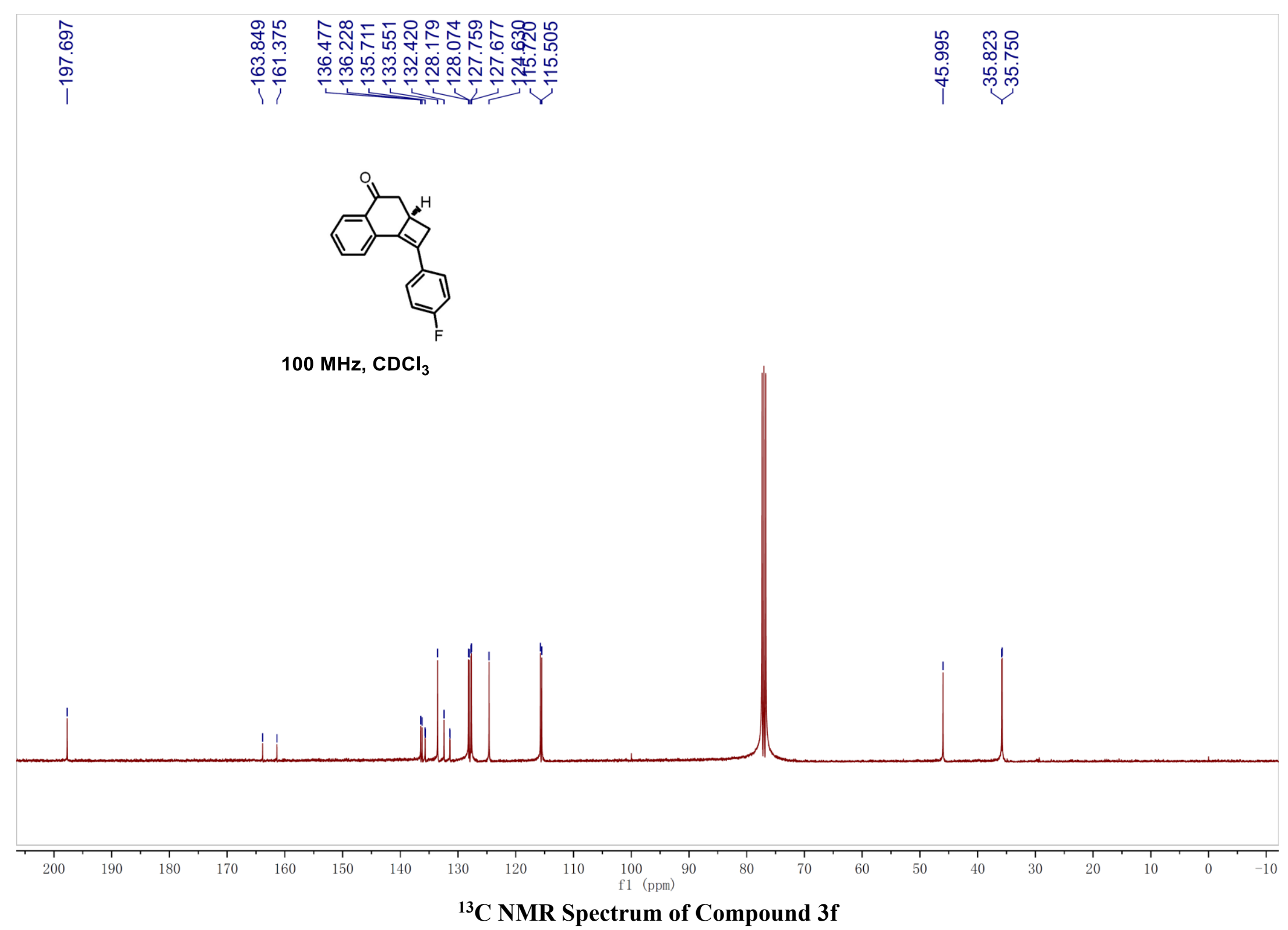




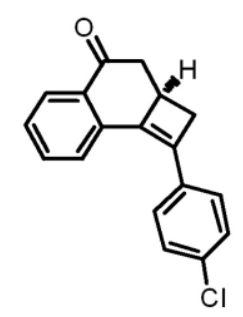

$400 \mathrm{MHz}^{\mathrm{CDCl}_{3}}$

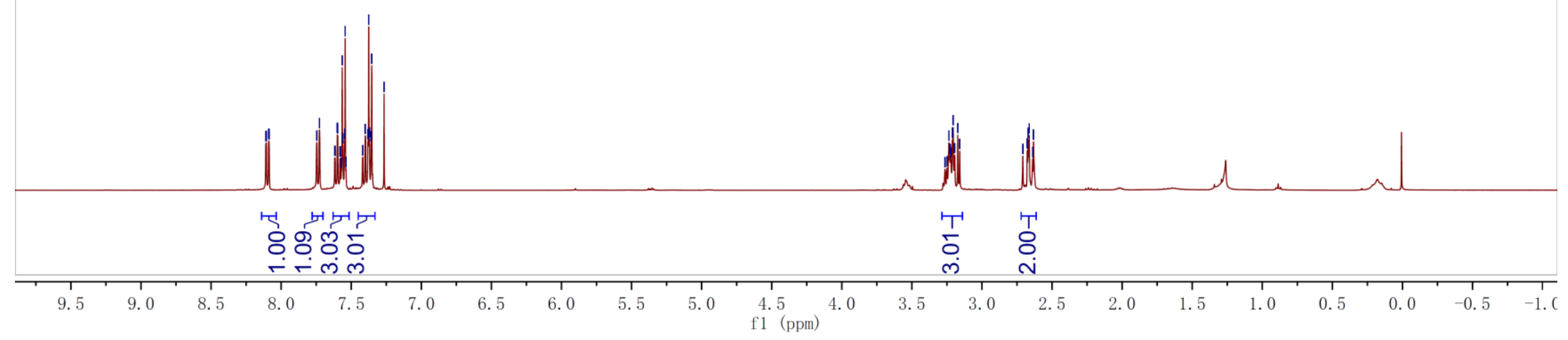

${ }^{1} \mathrm{H}$ NMR Spectrum of Compound $\mathbf{3 g}$ 


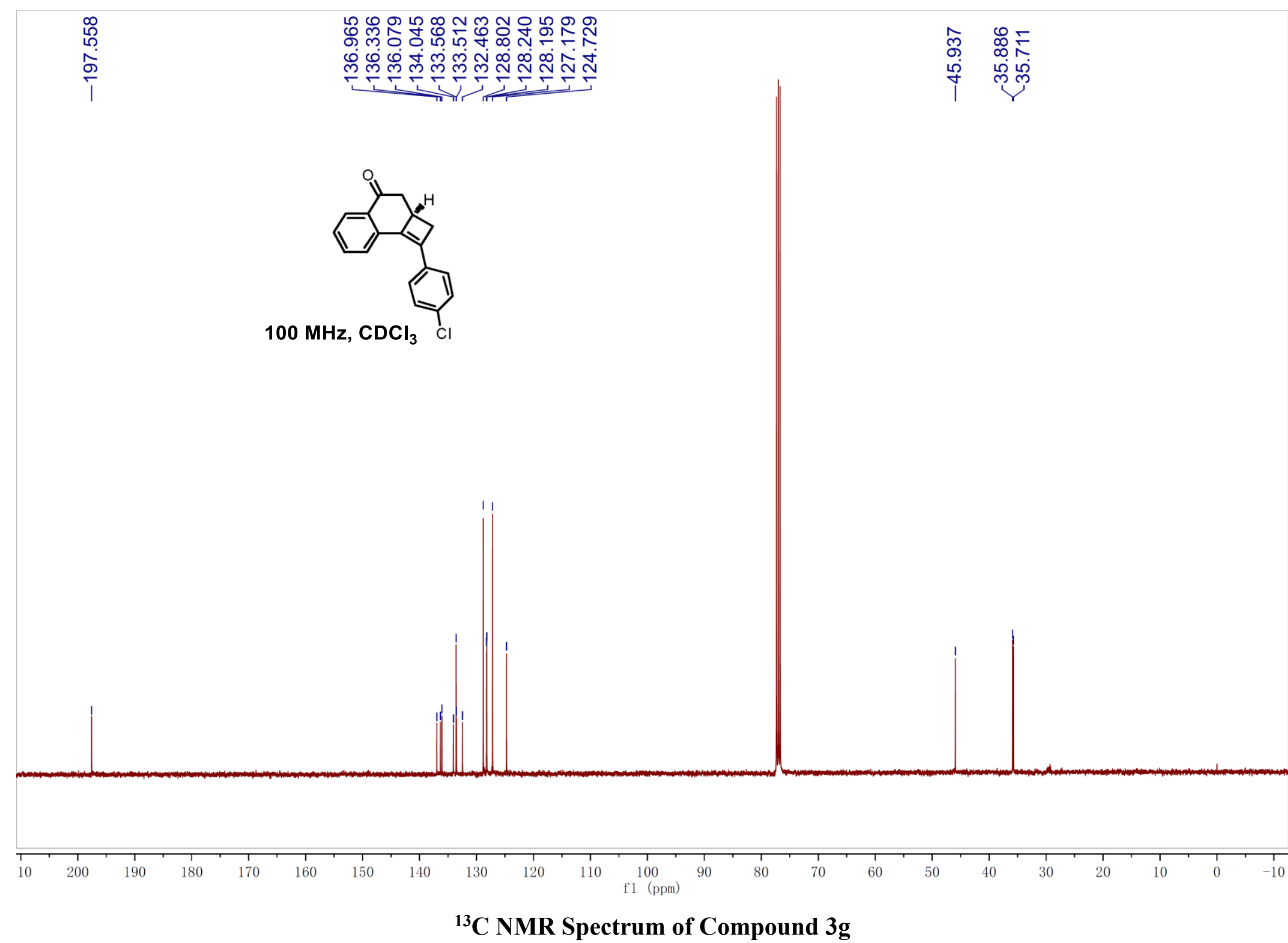




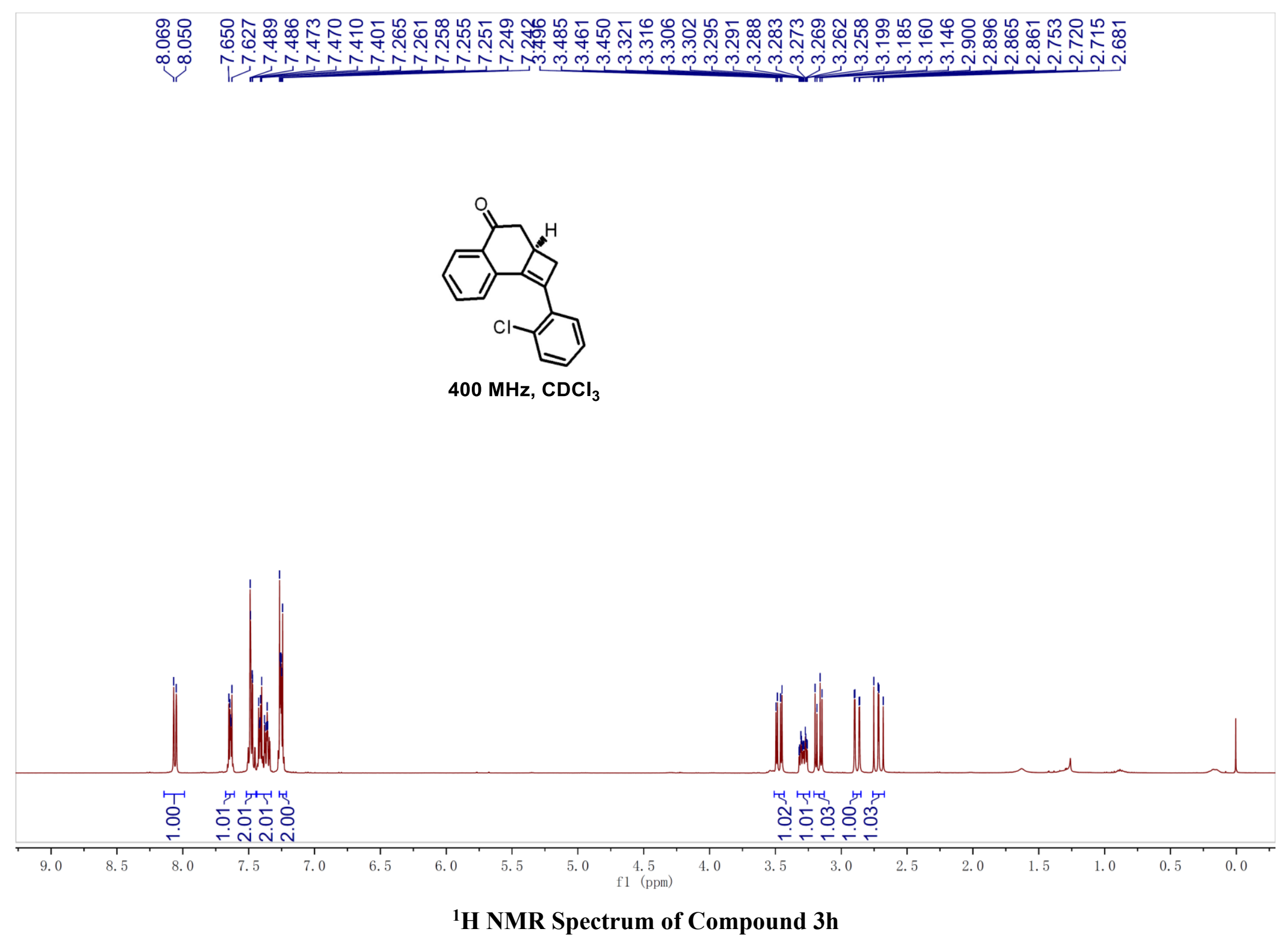




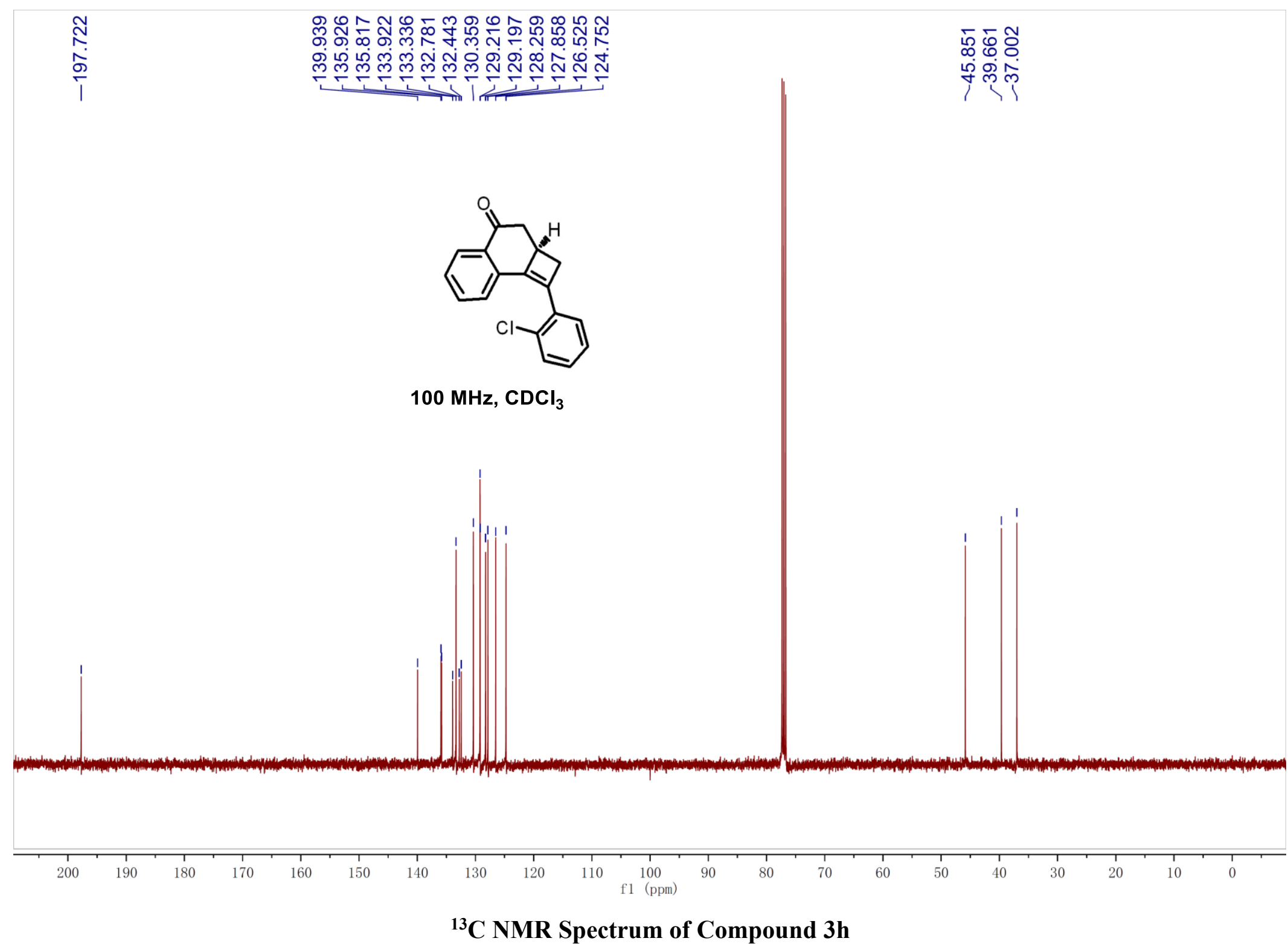




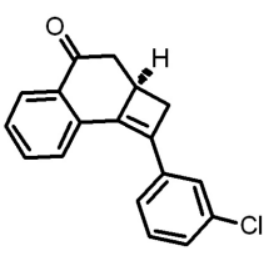

$400 \mathrm{MHz}, \mathrm{CDCl}_{3}$

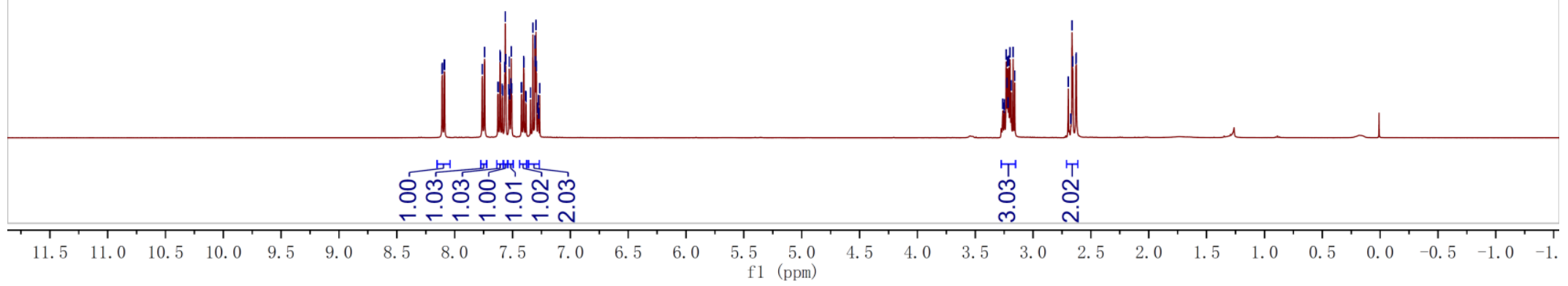

${ }^{1} \mathrm{H}$ NMR Spectrum of Compound 3i 


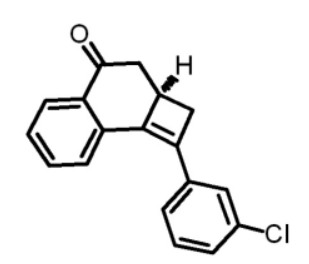

$100 \mathrm{MHz}, \mathrm{CDCl}_{3}$
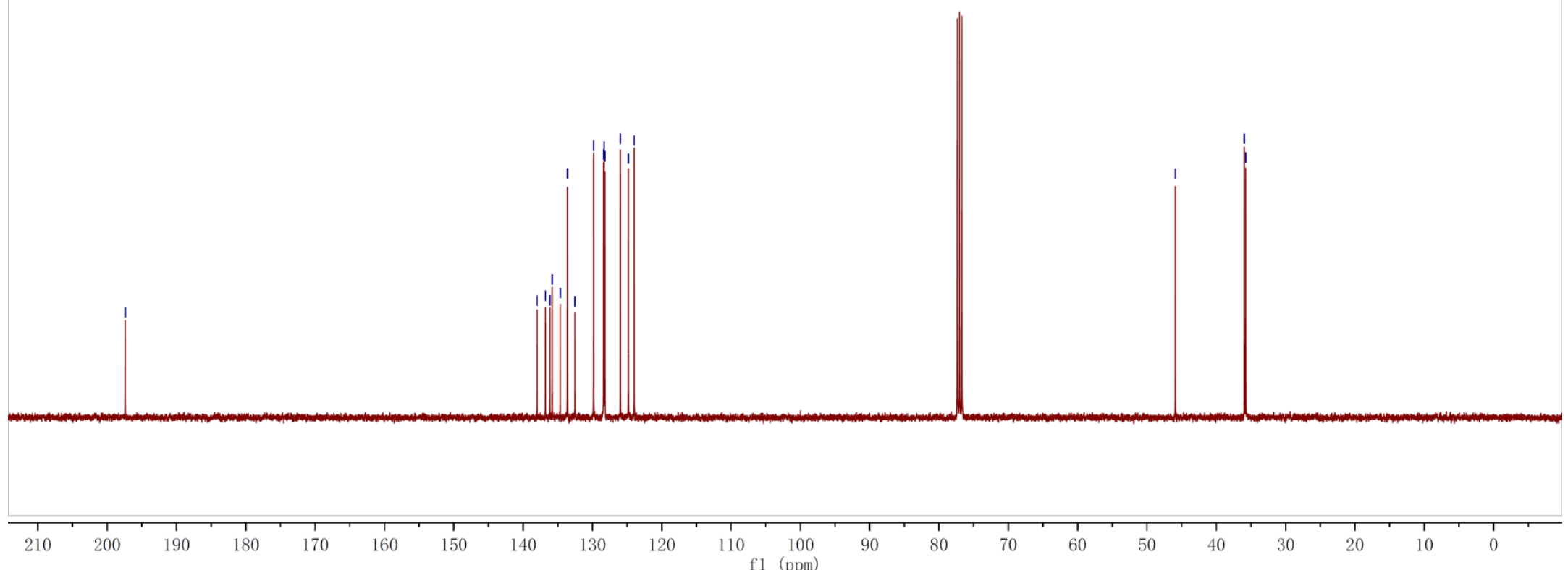

${ }^{13} \mathrm{C}$ NMR Spectrum of Compound 3i 


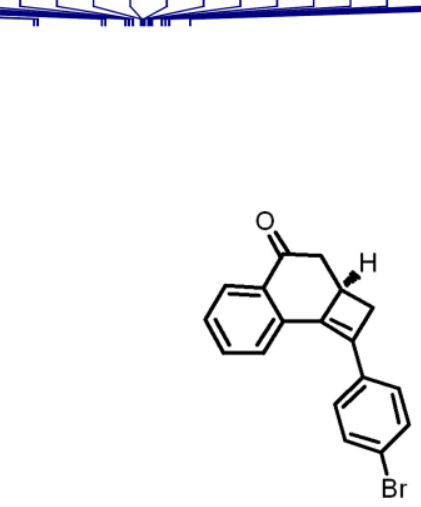

$400 \mathrm{MHz}^{\mathrm{CDCl}_{3}}$

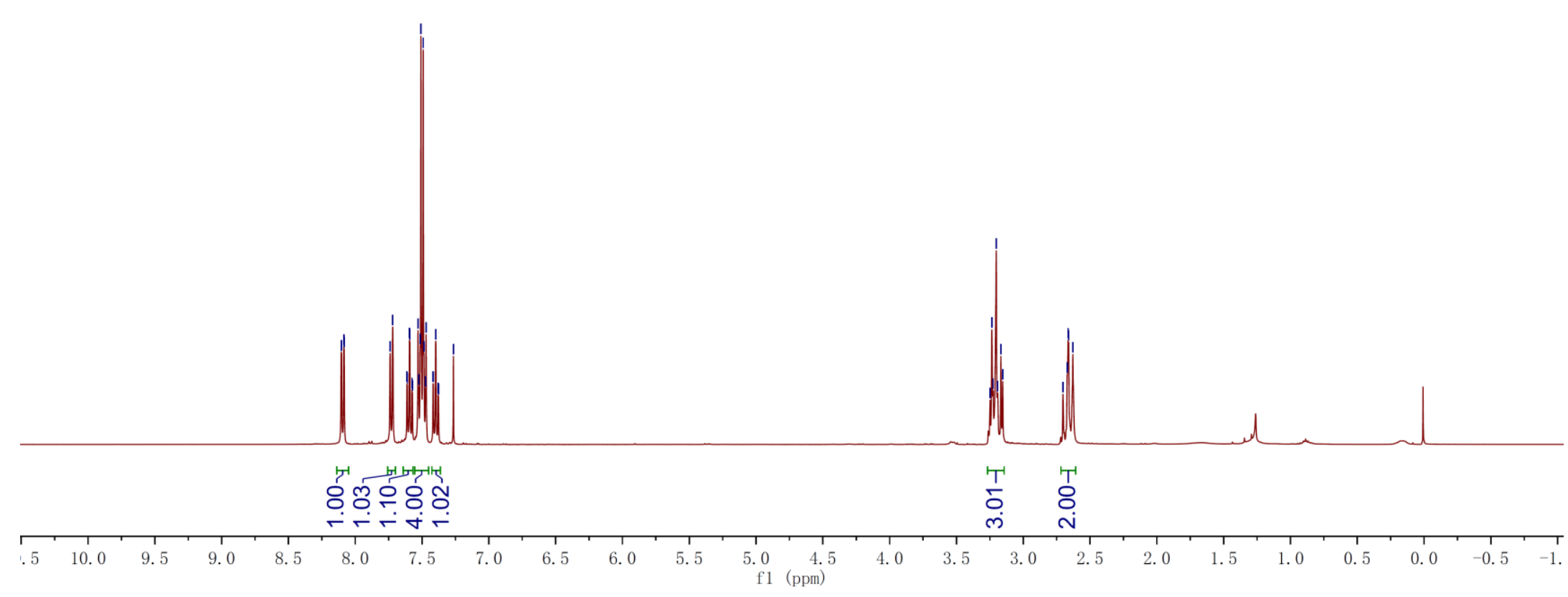

${ }^{1}$ H NMR Spectrum of Compound $3 \mathbf{j}$ 


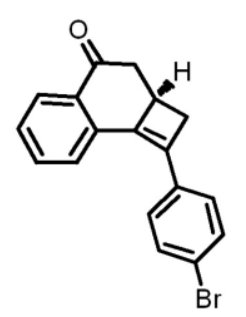

$100 \mathrm{MHz}, \mathrm{CDCl}_{3}$
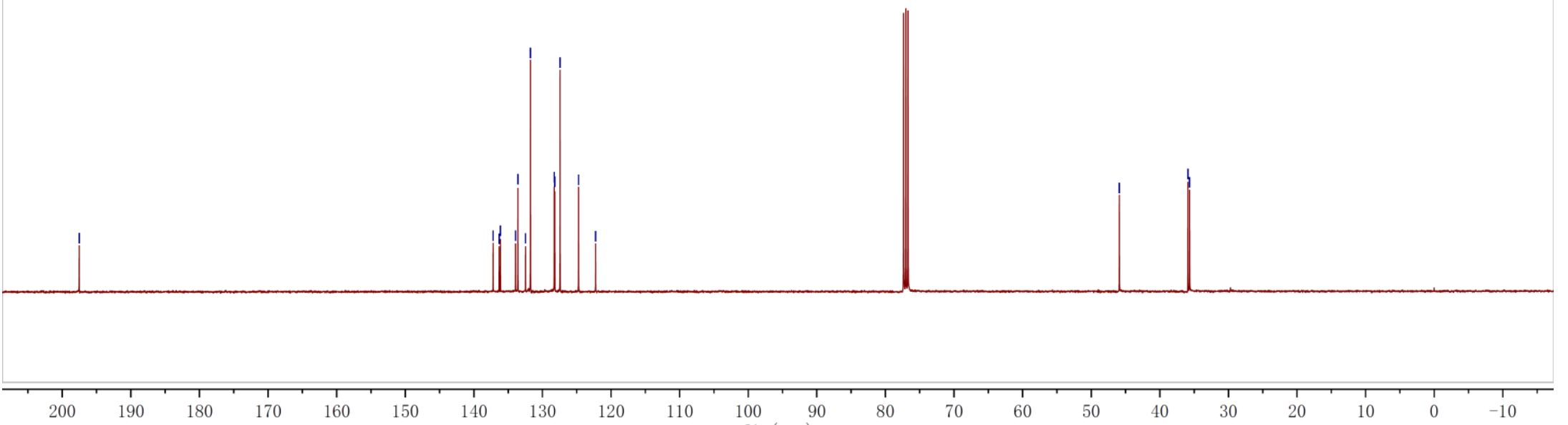

\section{${ }^{13} \mathrm{C}$ NMR Spectrum of Compound $3 \mathrm{j}$}



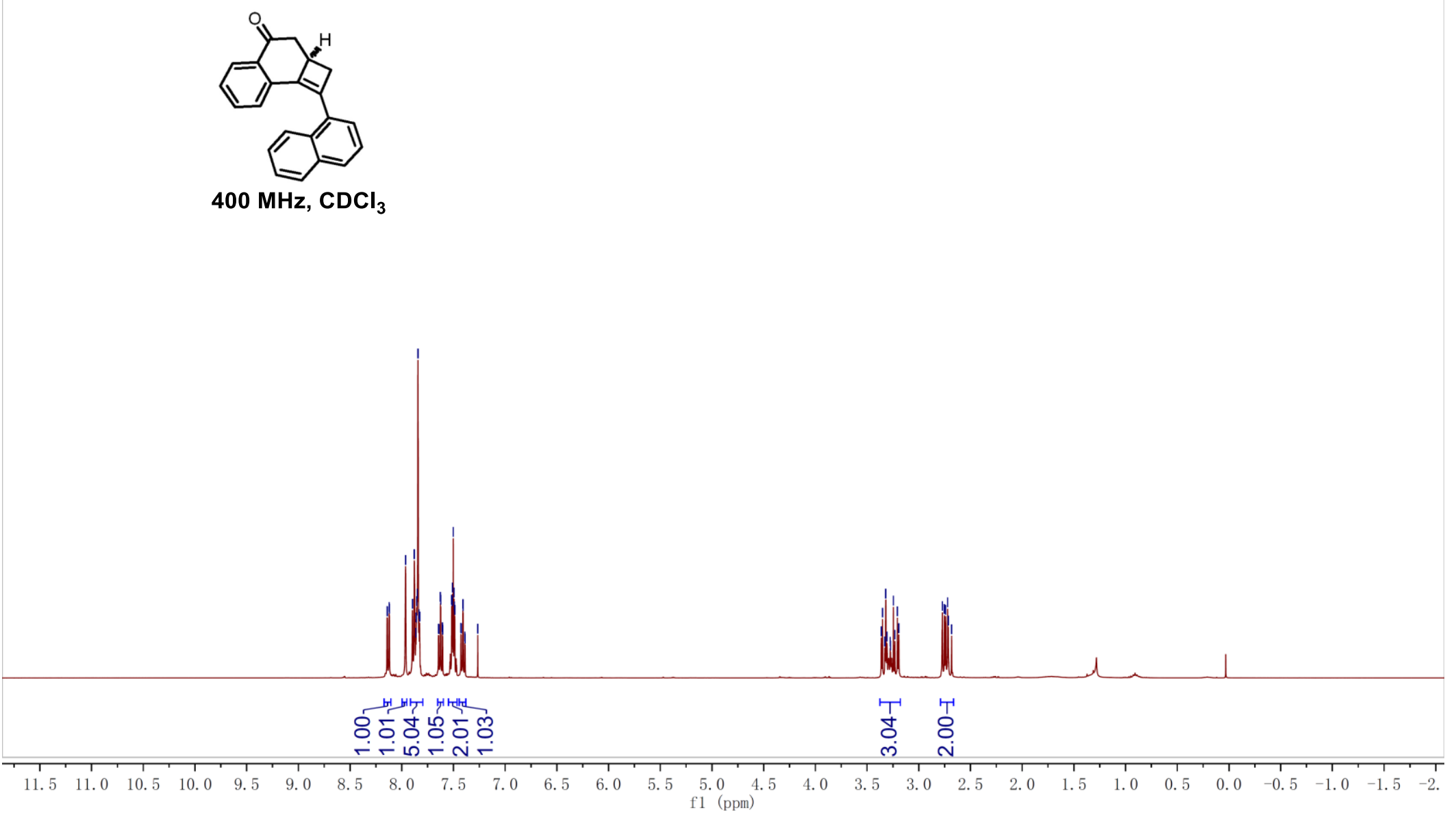

${ }^{1}$ H NMR Spectrum of Compound 3k 


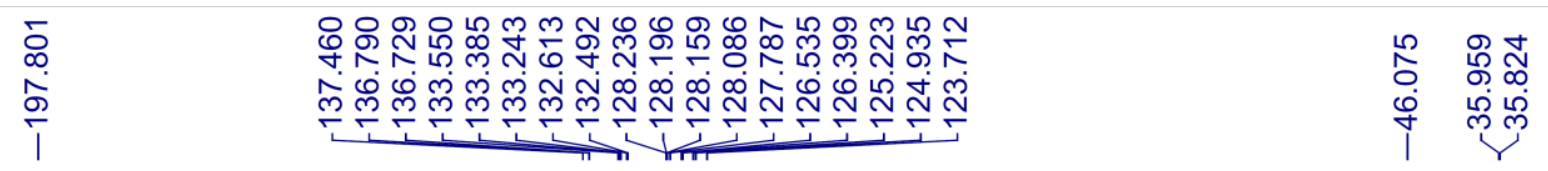

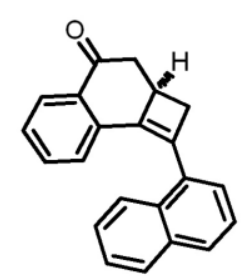

$100 \mathrm{MHz}^{\mathrm{CDCl}_{3}}$
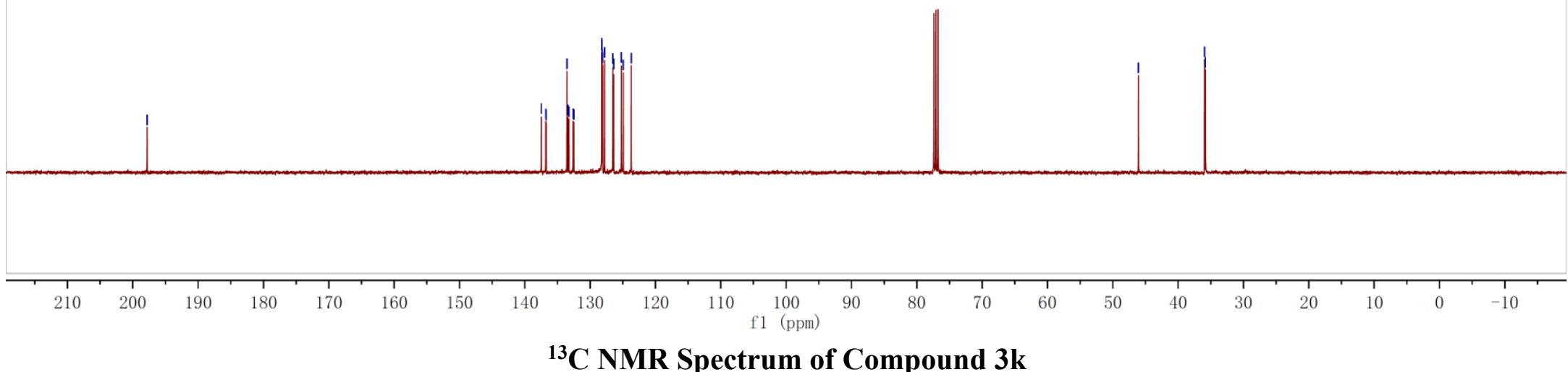


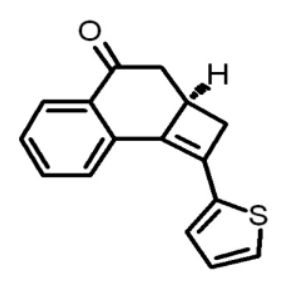

$400 \mathrm{MHz}^{\mathrm{CDCl}_{3}}$

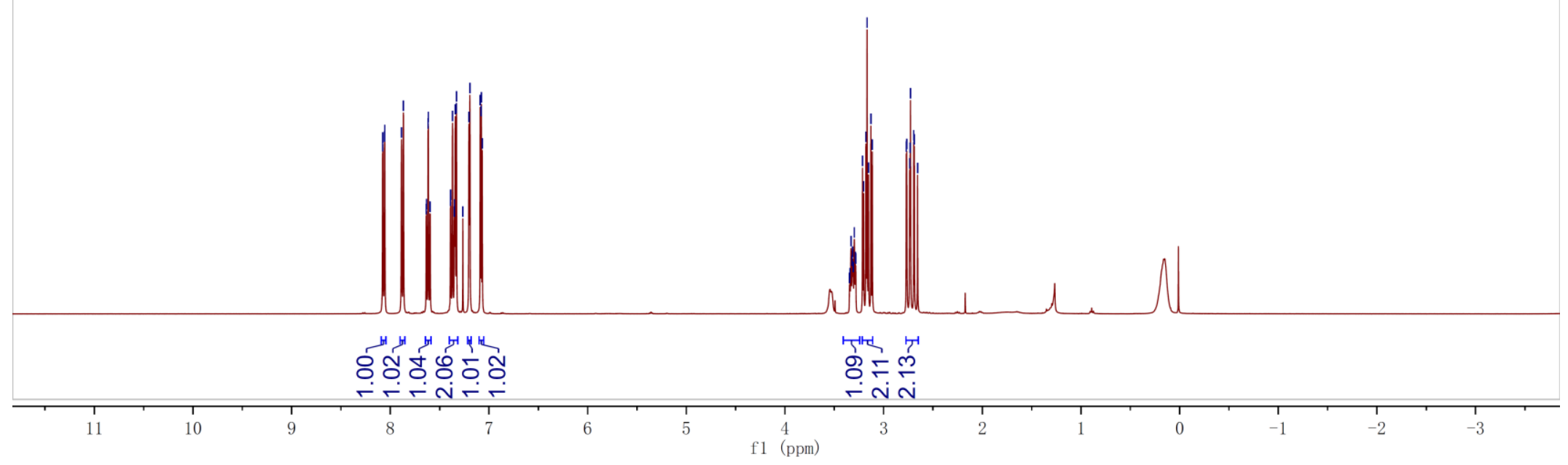

${ }^{1}$ H NMR Spectrum of Compound 3I 


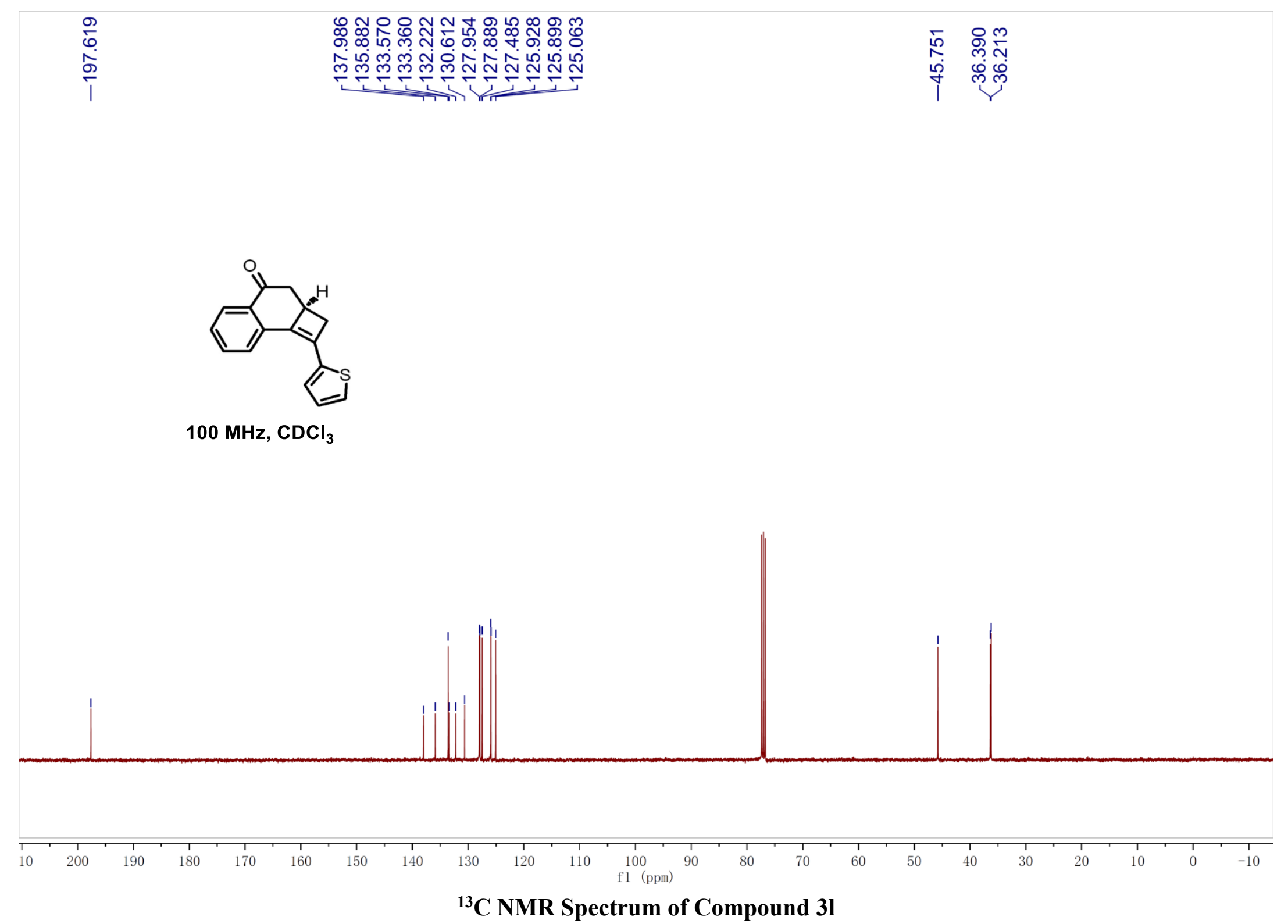




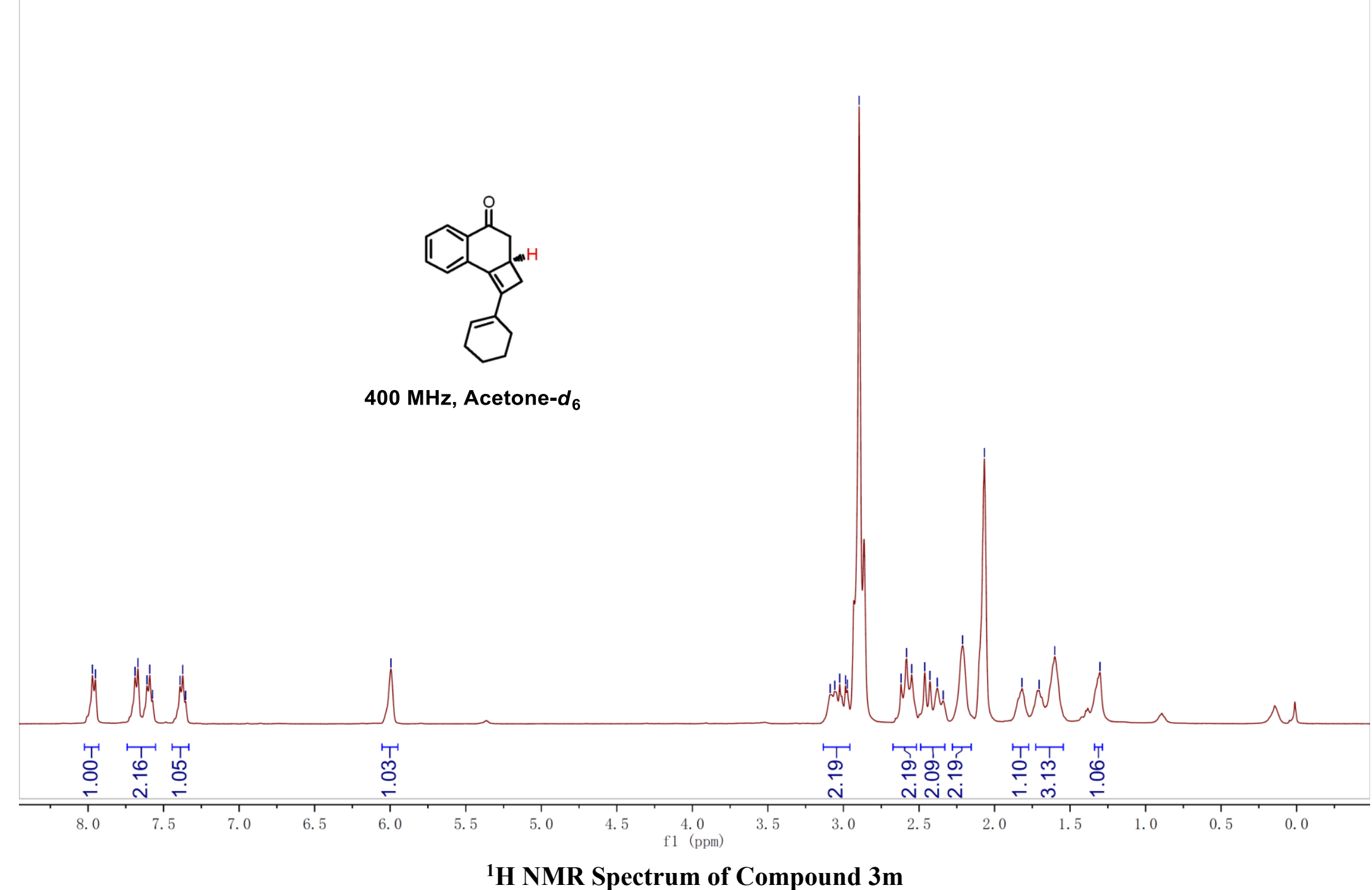




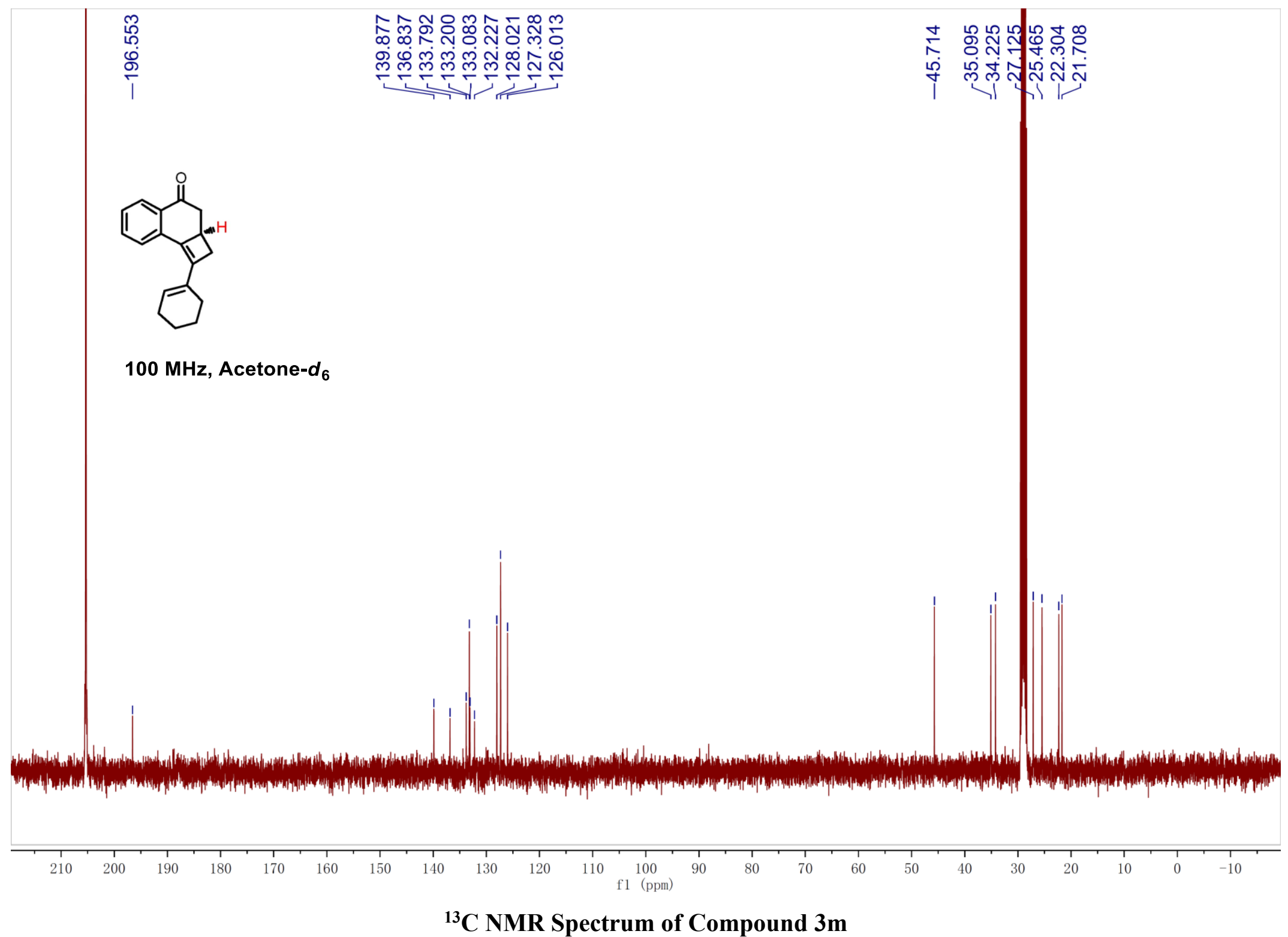




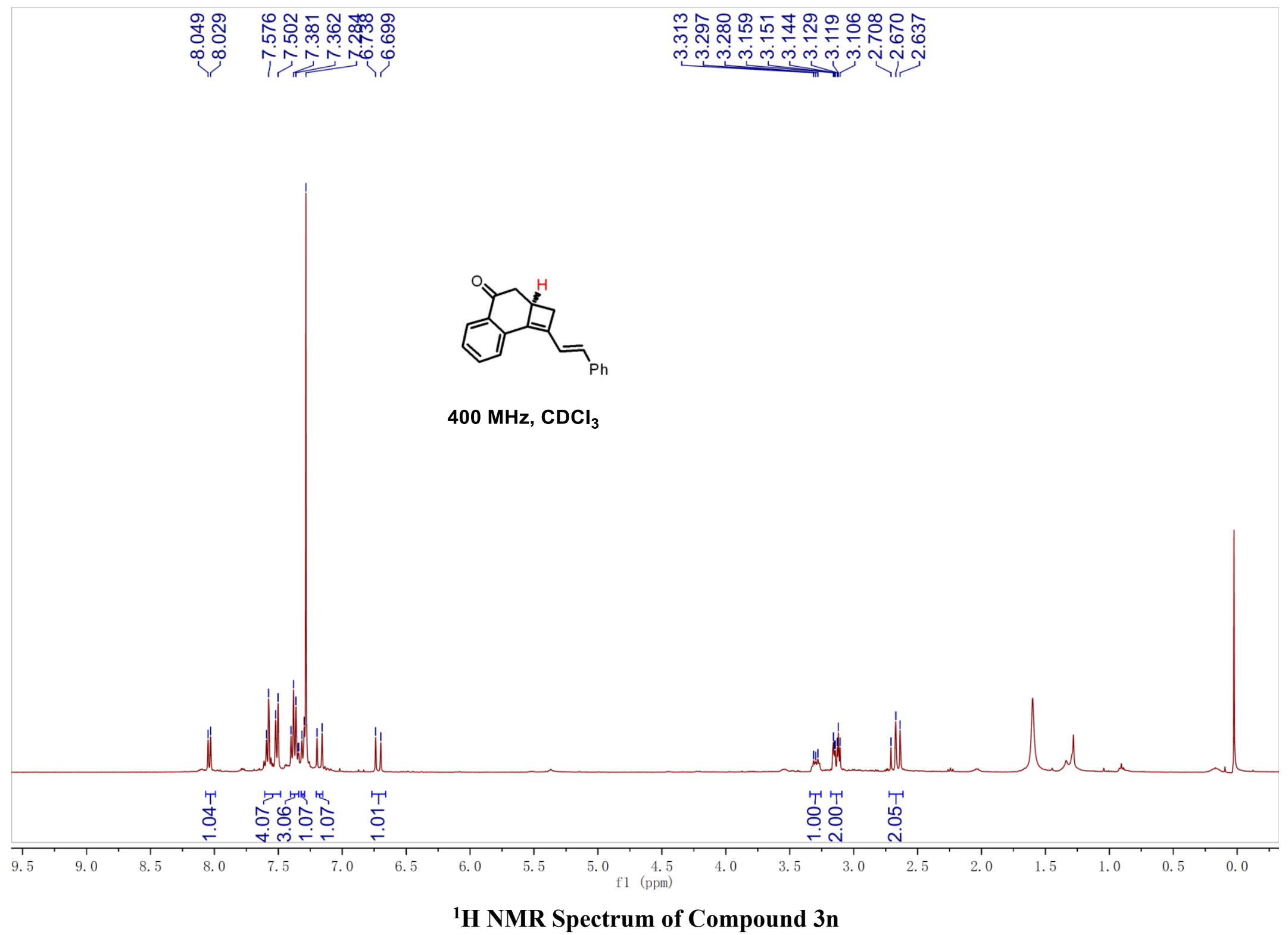




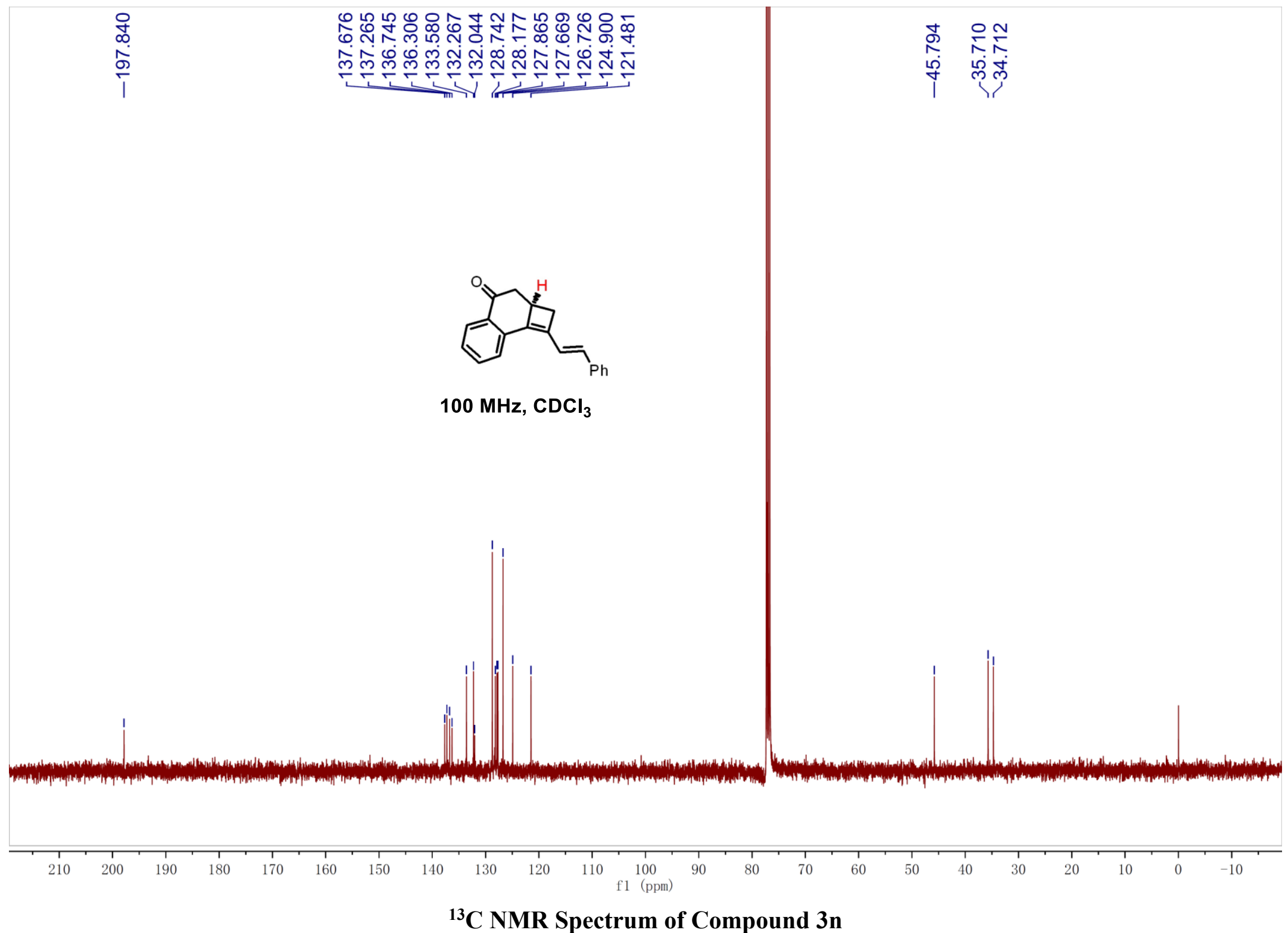




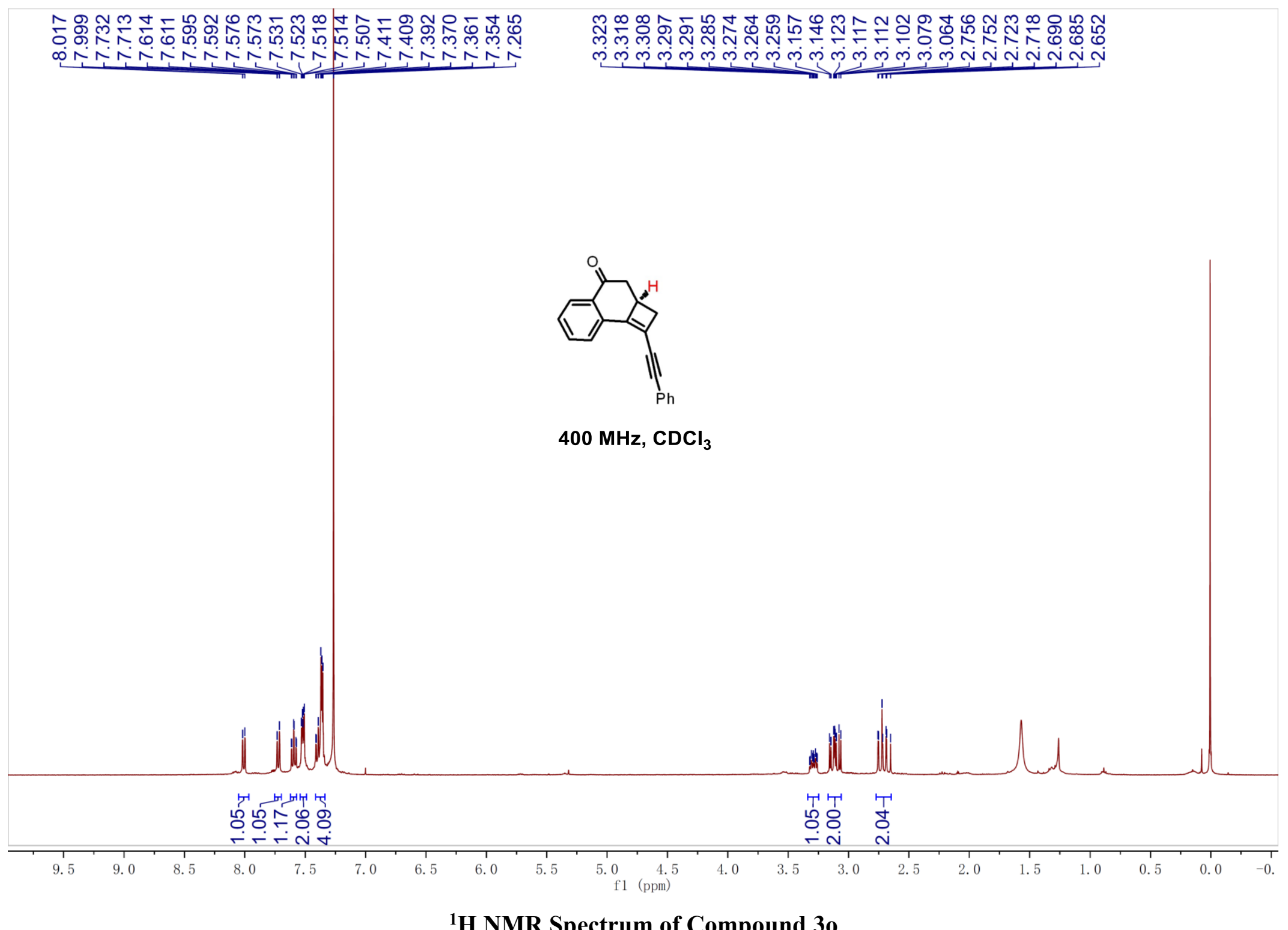

${ }^{1} \mathrm{H}$ NMR Spectrum of Compound $3 \mathrm{o}$ 


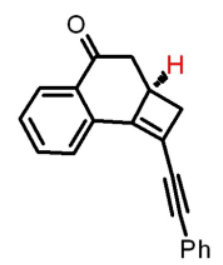

$100 \mathrm{MHz}, \mathrm{CDCl}_{3}$
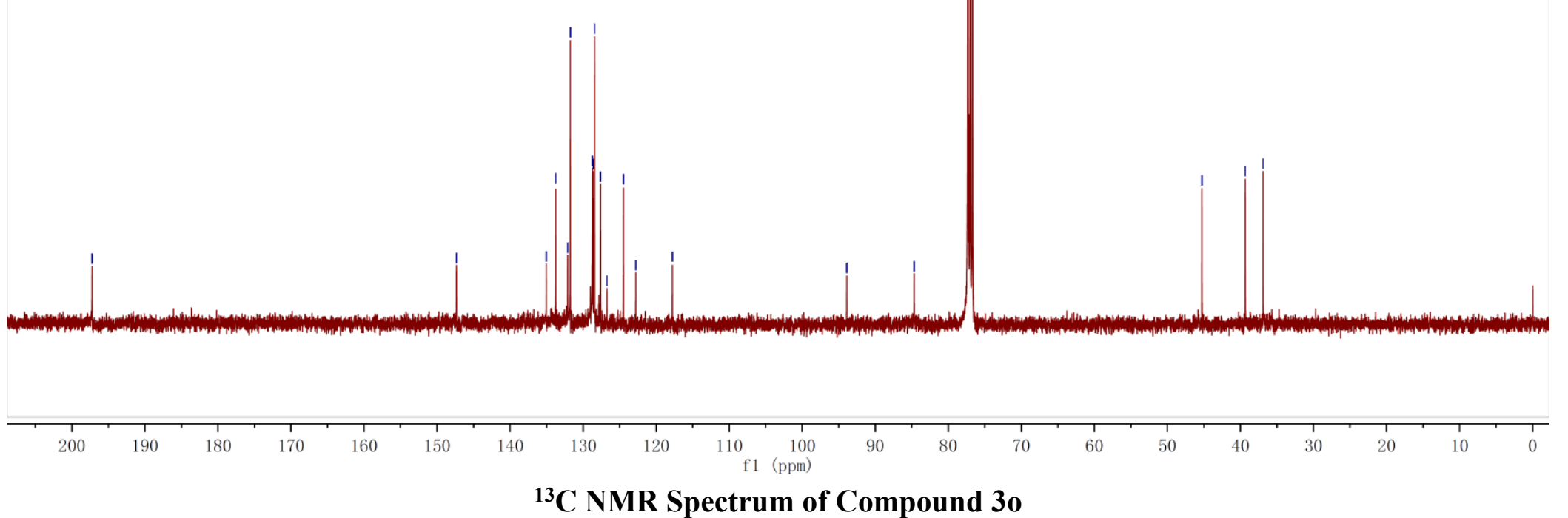


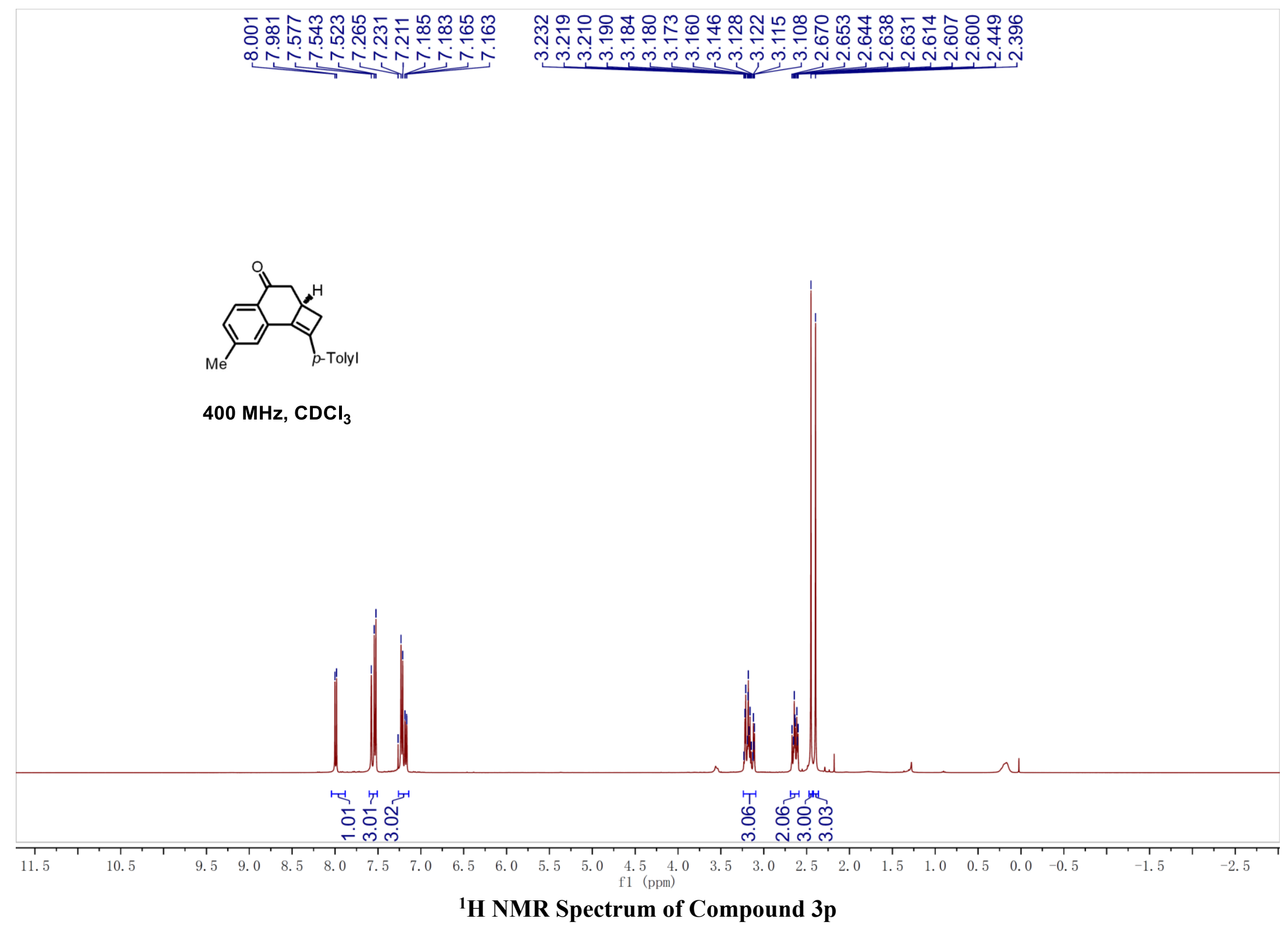



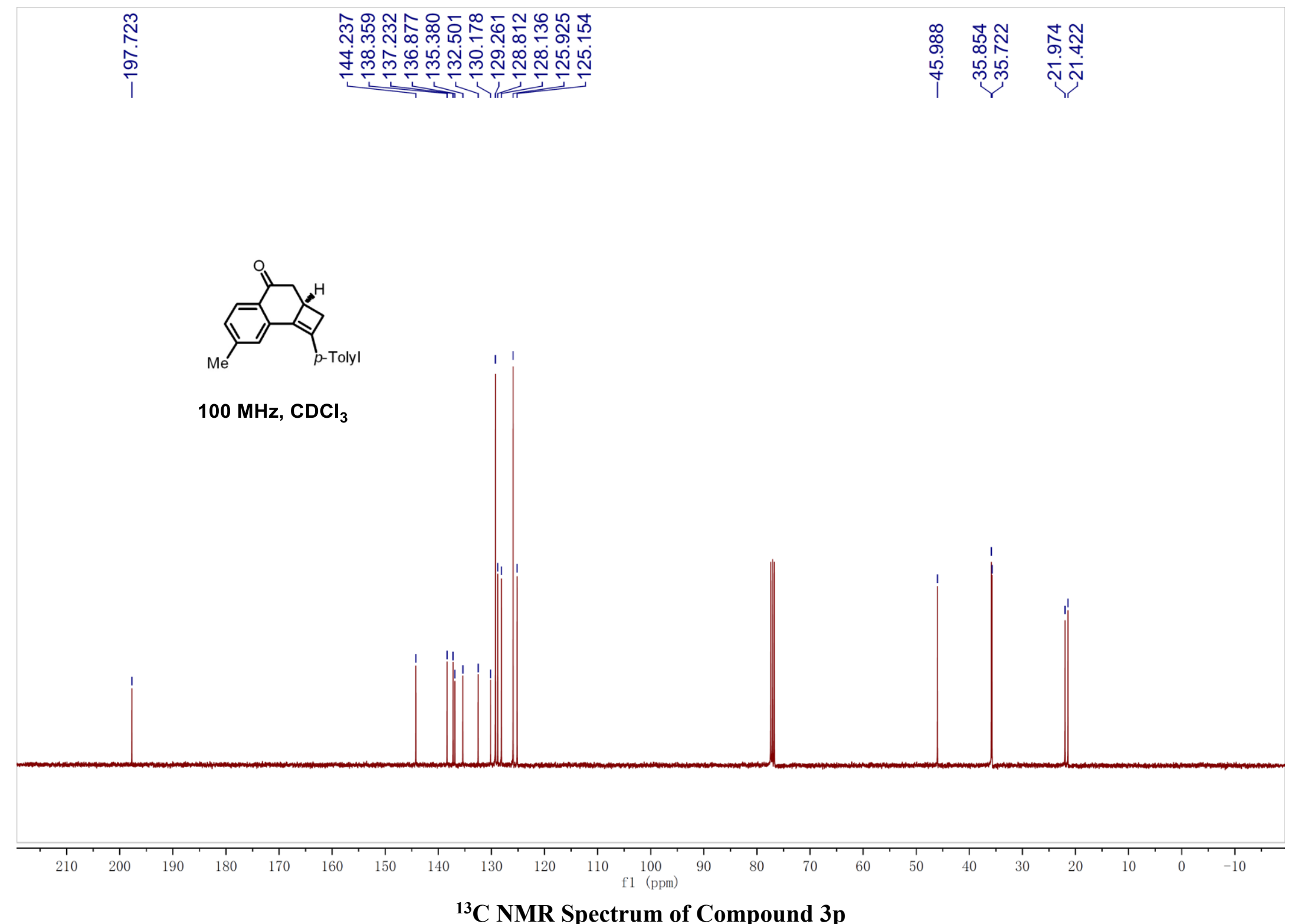


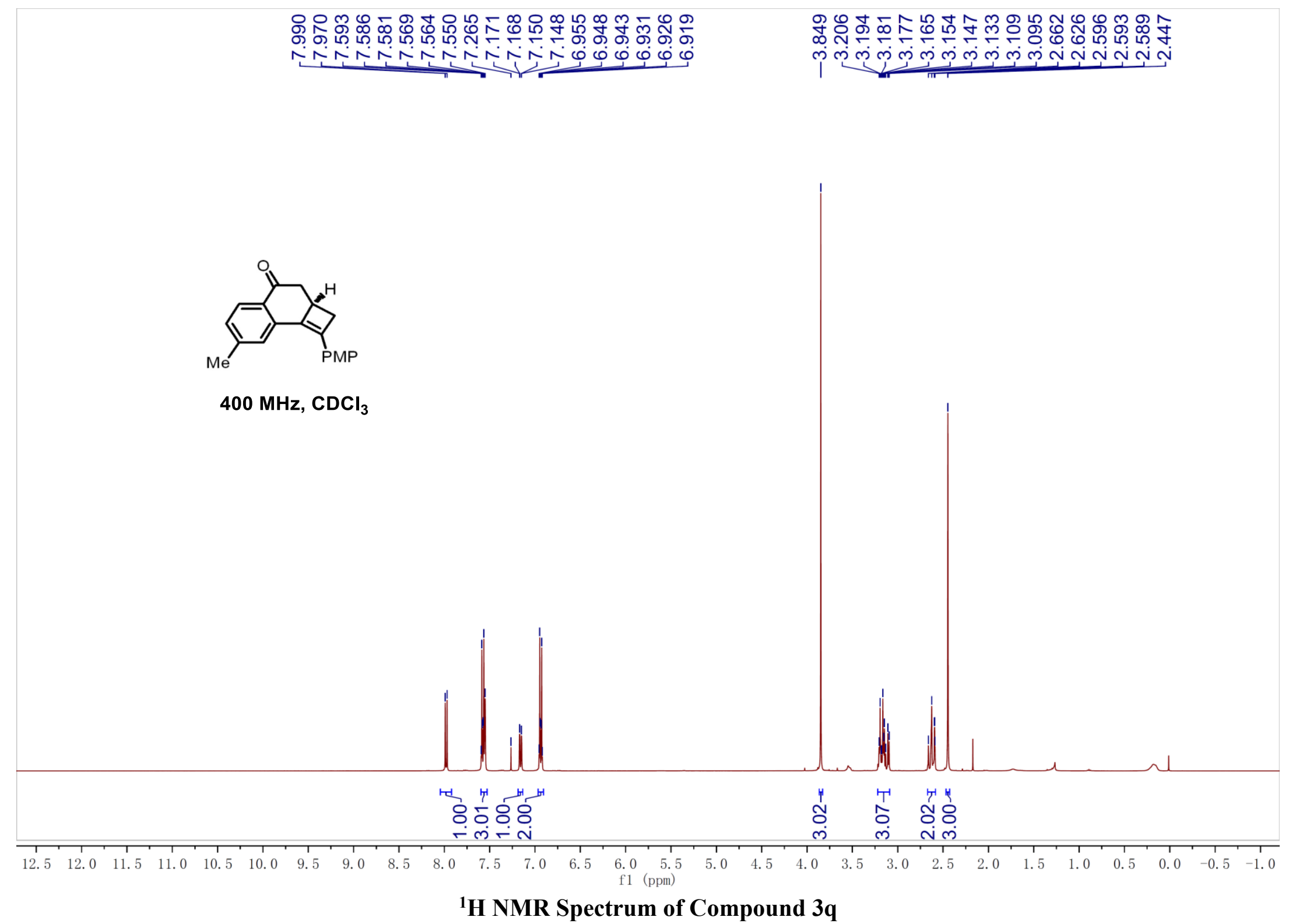



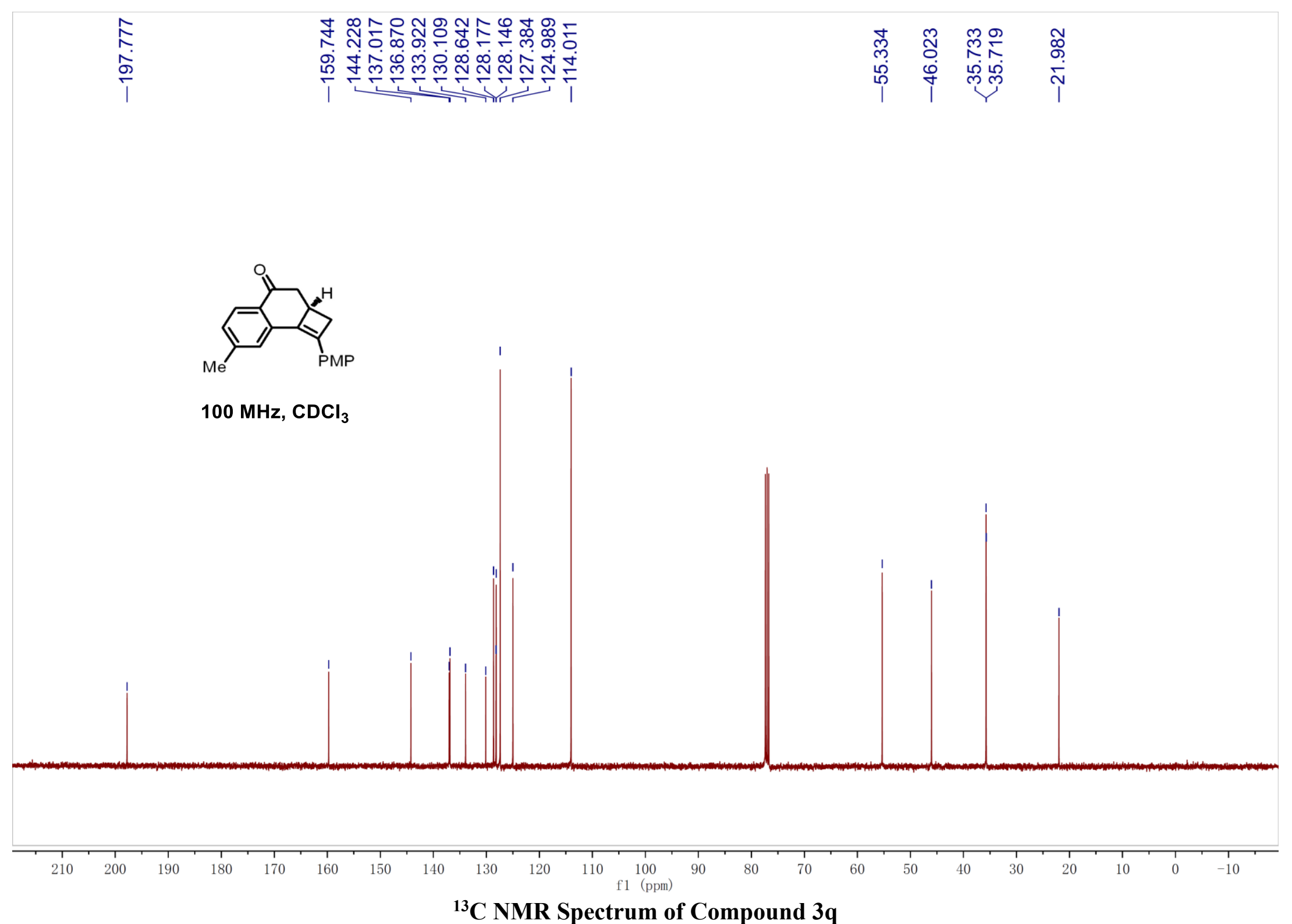


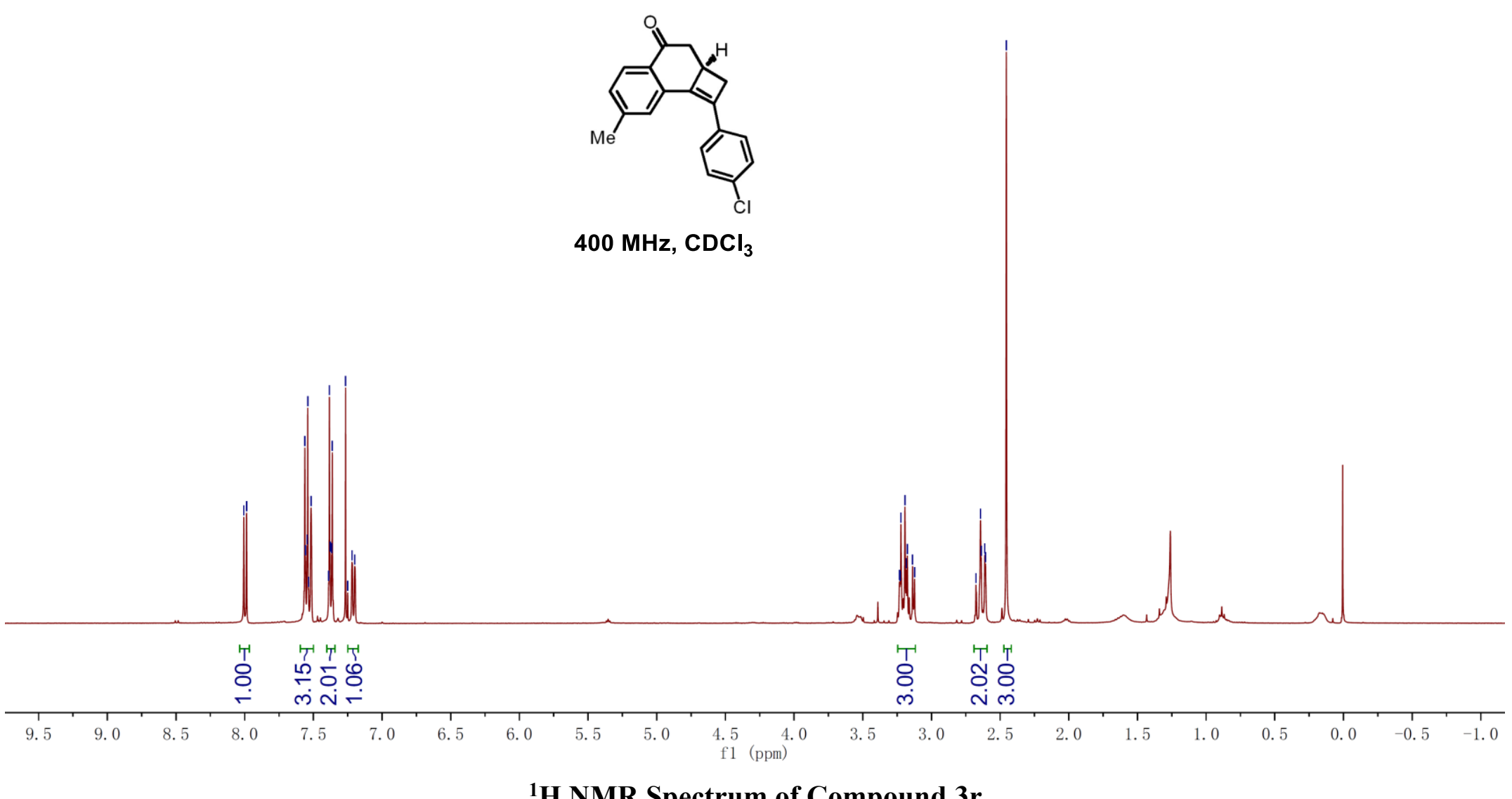



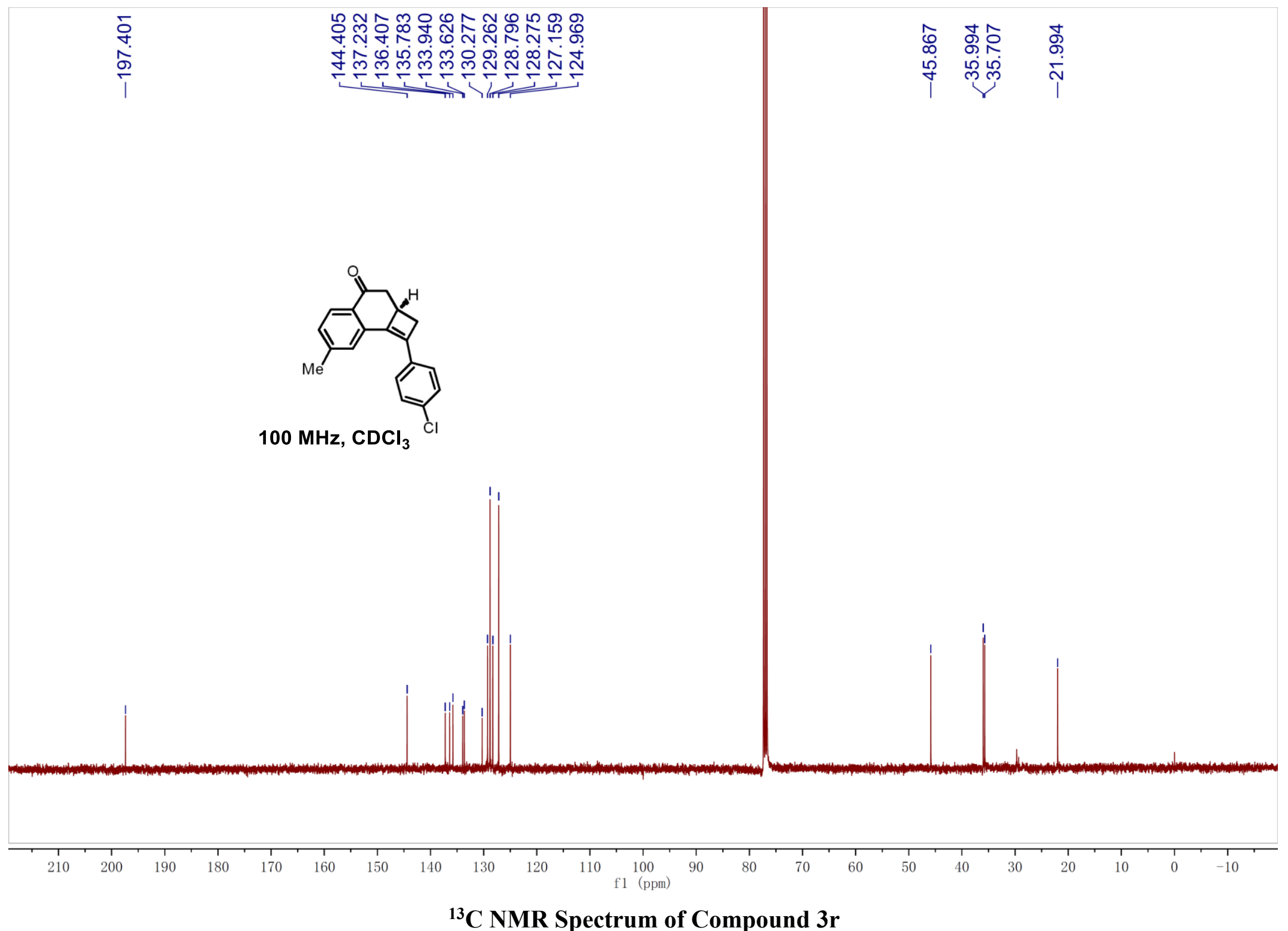


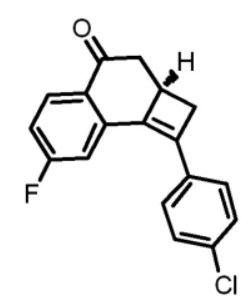

$400 \mathrm{MHz}^{\mathrm{CDCl}_{3}}$

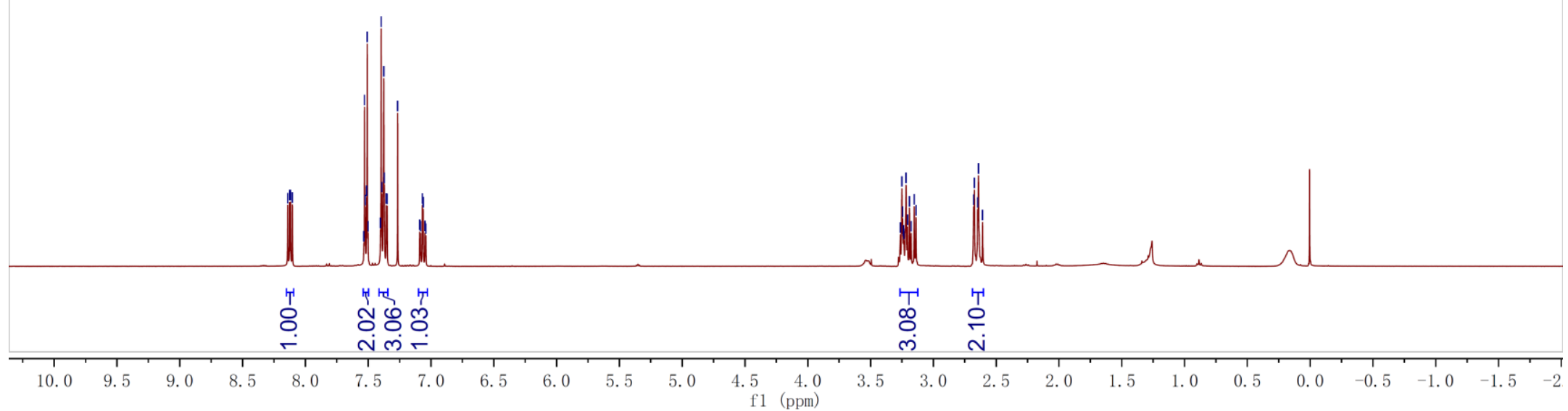

${ }^{1}$ H NMR Spectrum of Compound 3s 


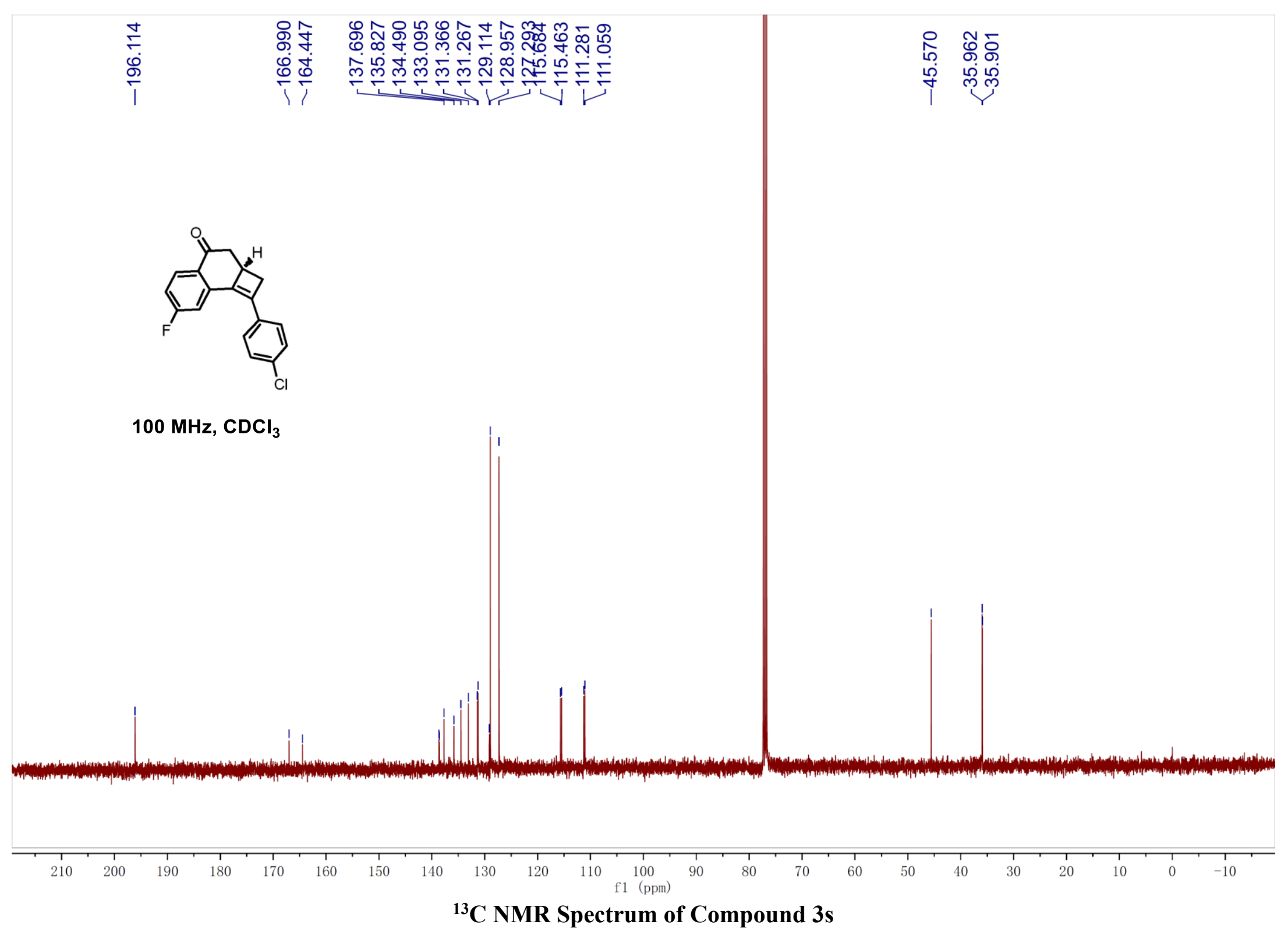




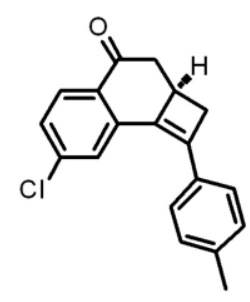

$400 \mathrm{MHz}, \mathrm{CDCl}_{3}$

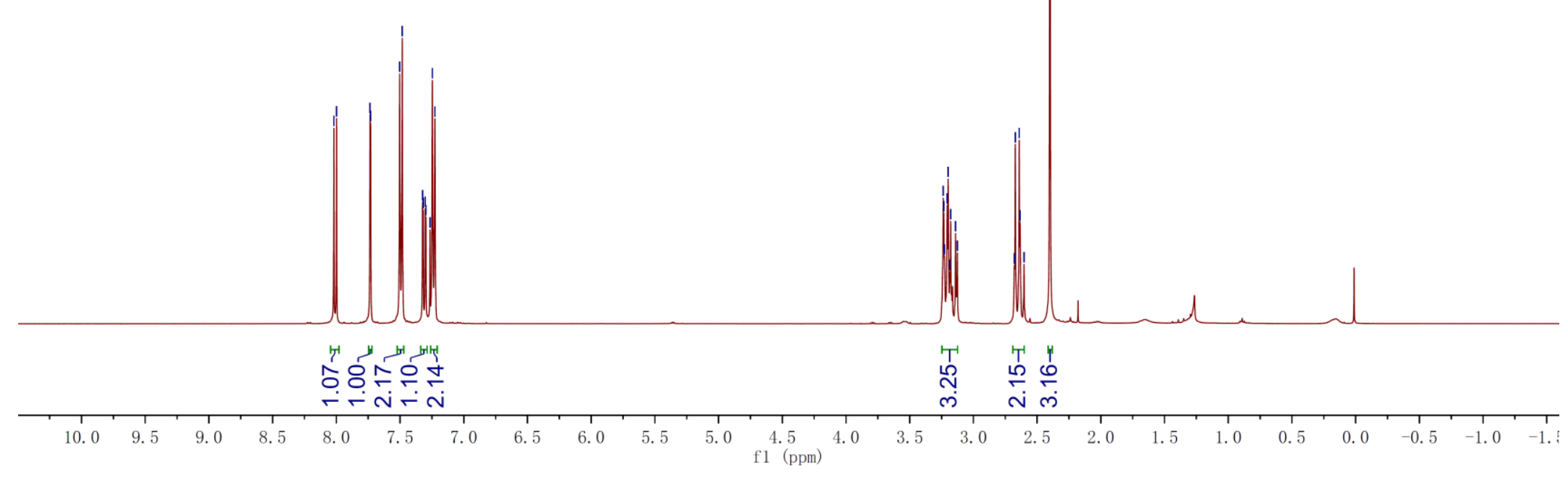

${ }^{1} \mathrm{H}$ NMR Spectrum of Compound $3 \mathrm{t}$ 

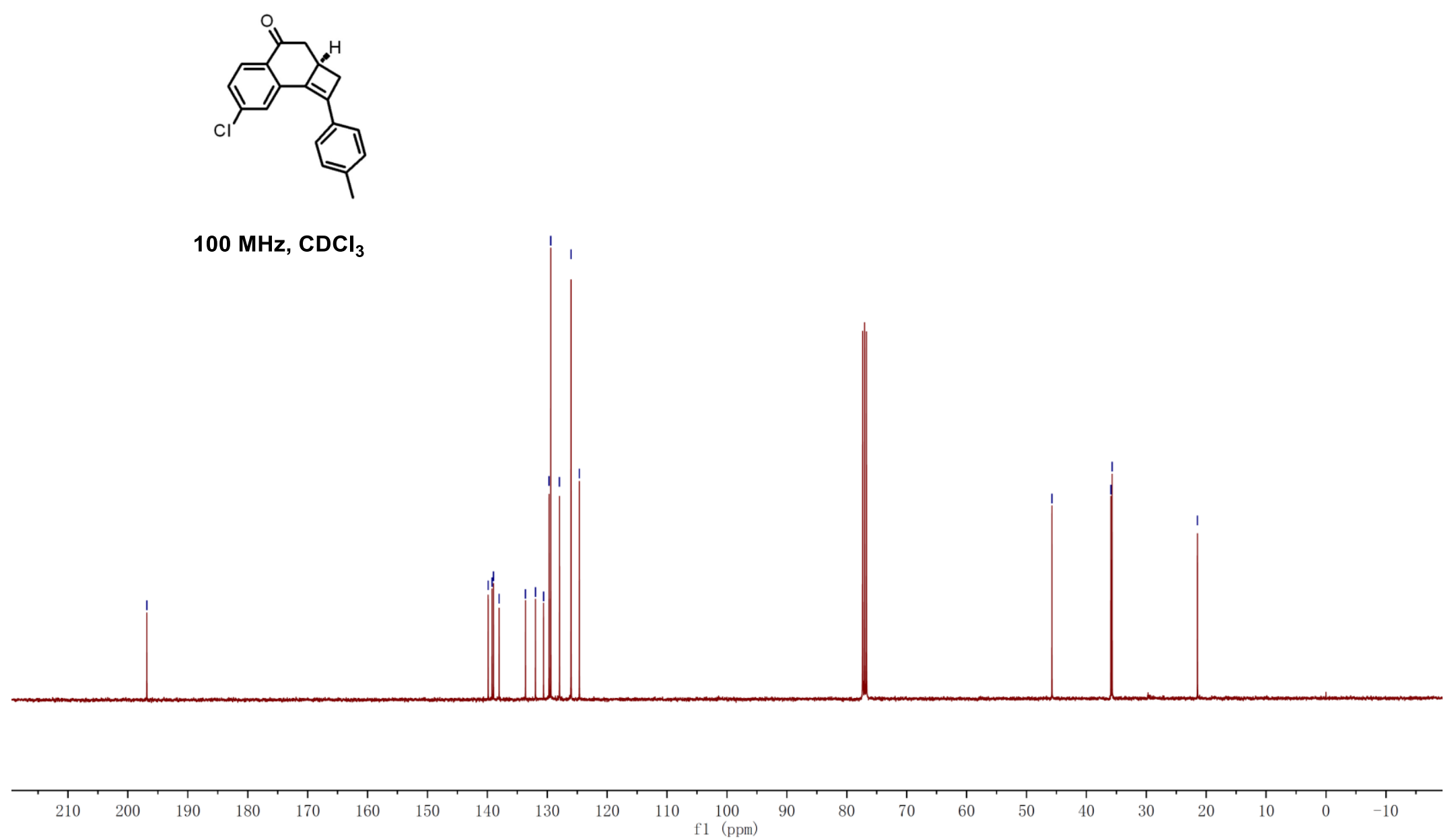

${ }^{13}$ C NMR Spectrum of Compound 3t 

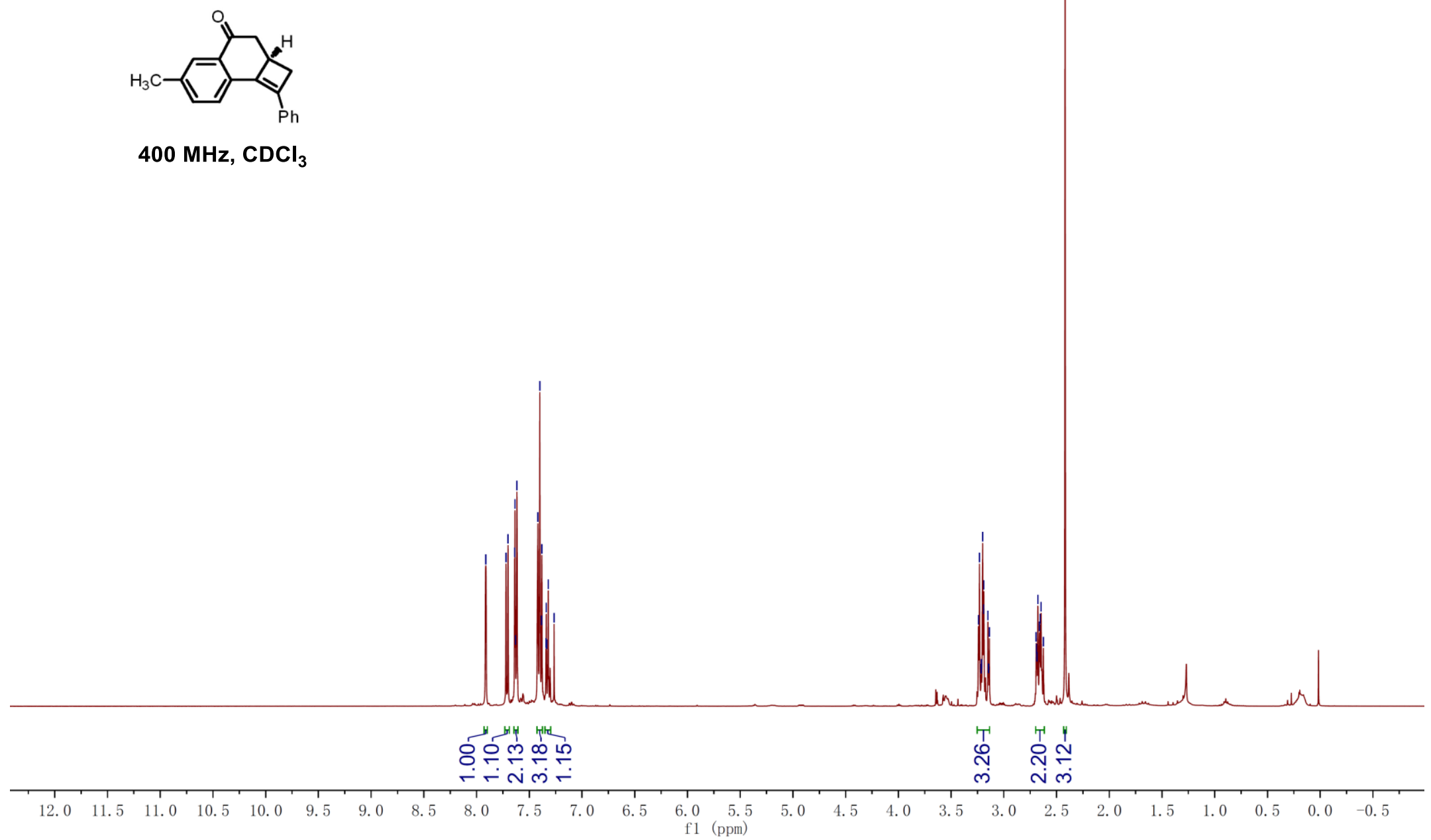

${ }^{1}$ H NMR Spectrum of Compound 3u 


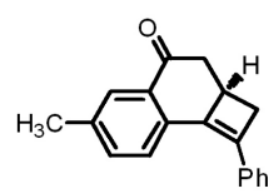

$100 \mathrm{MHz}, \mathrm{CDCl}_{3}$

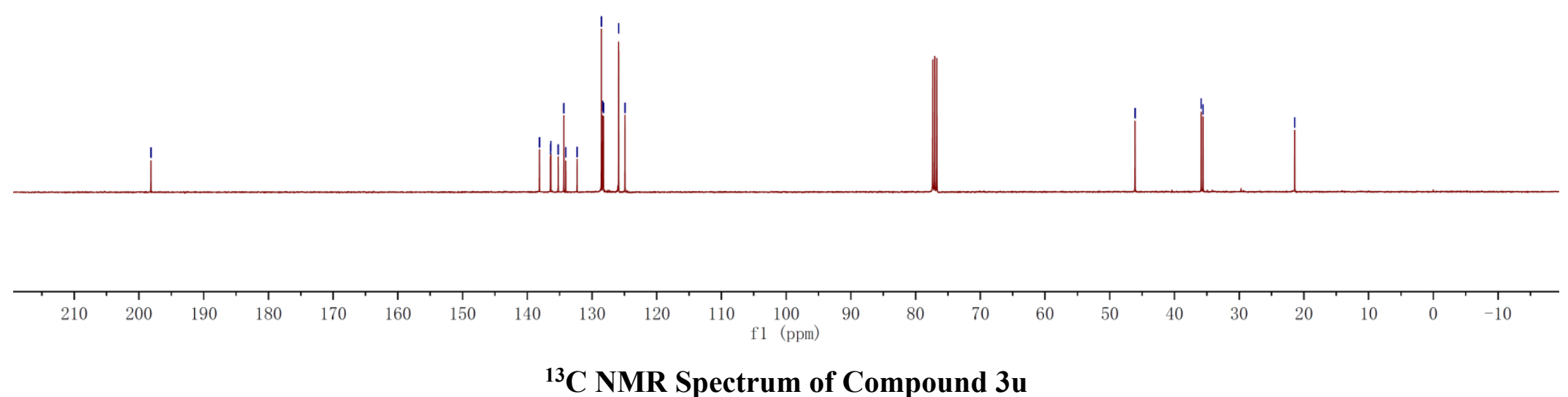




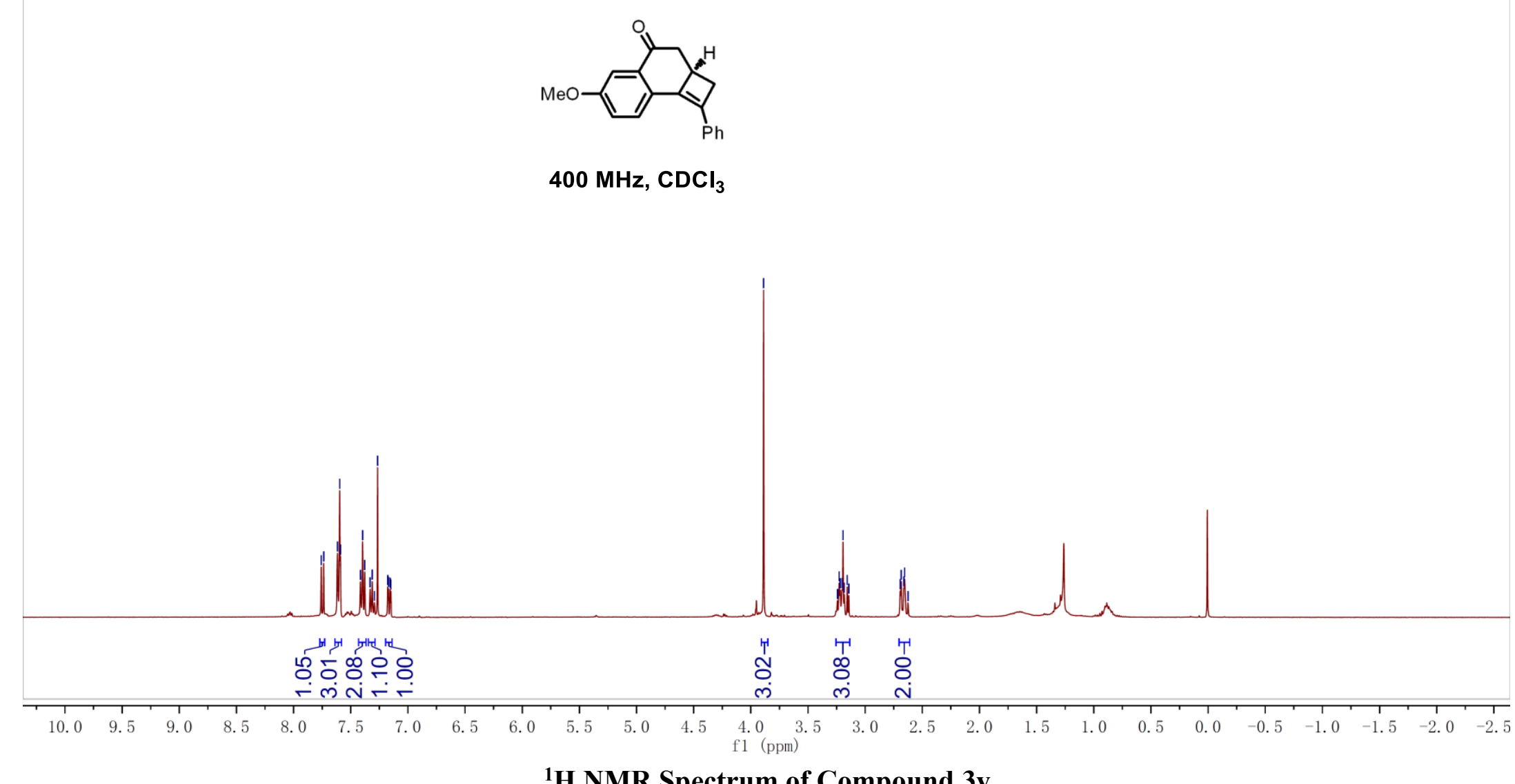

${ }^{1}$ H NMR Spectrum of Compound 3v 


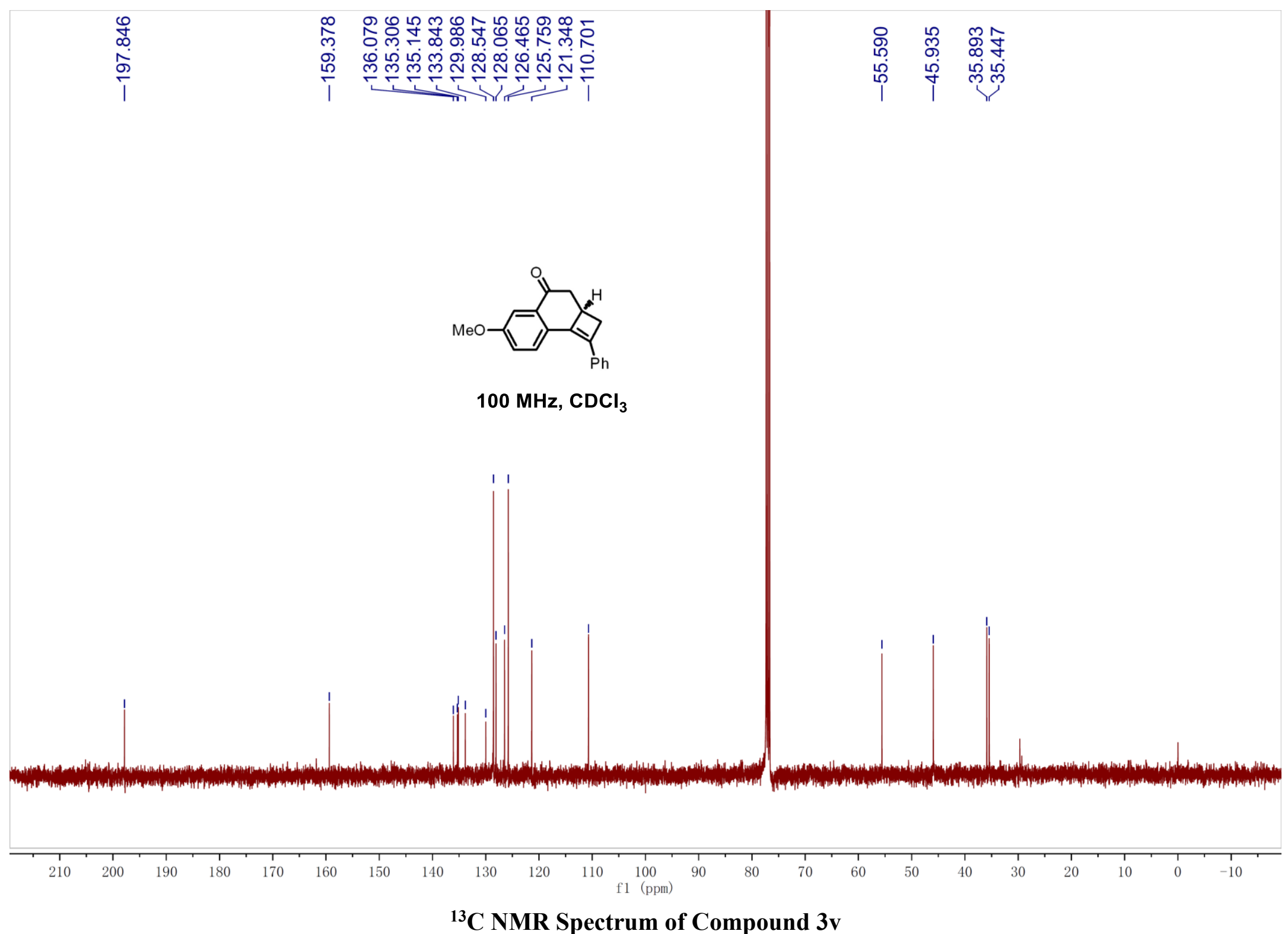




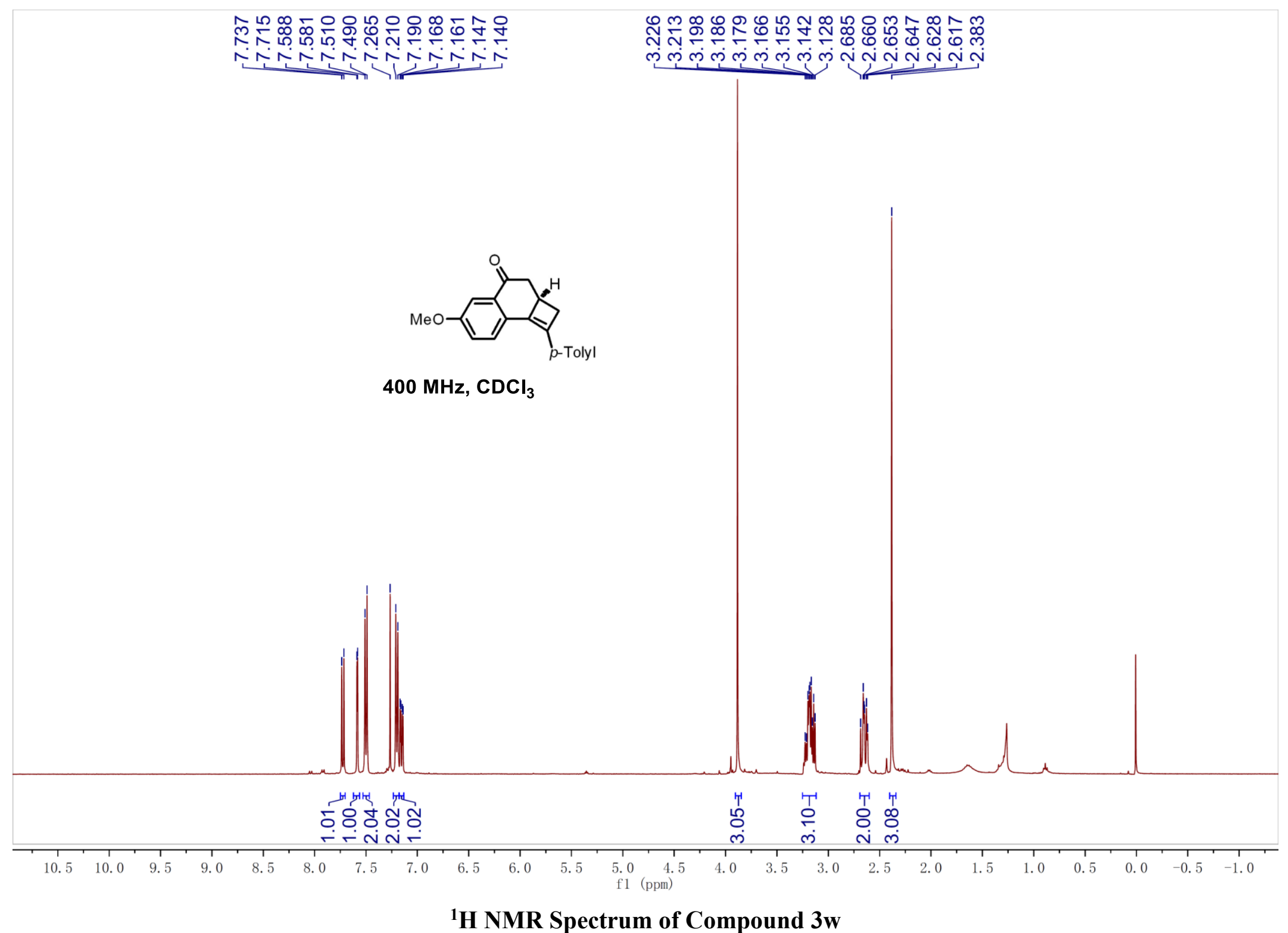



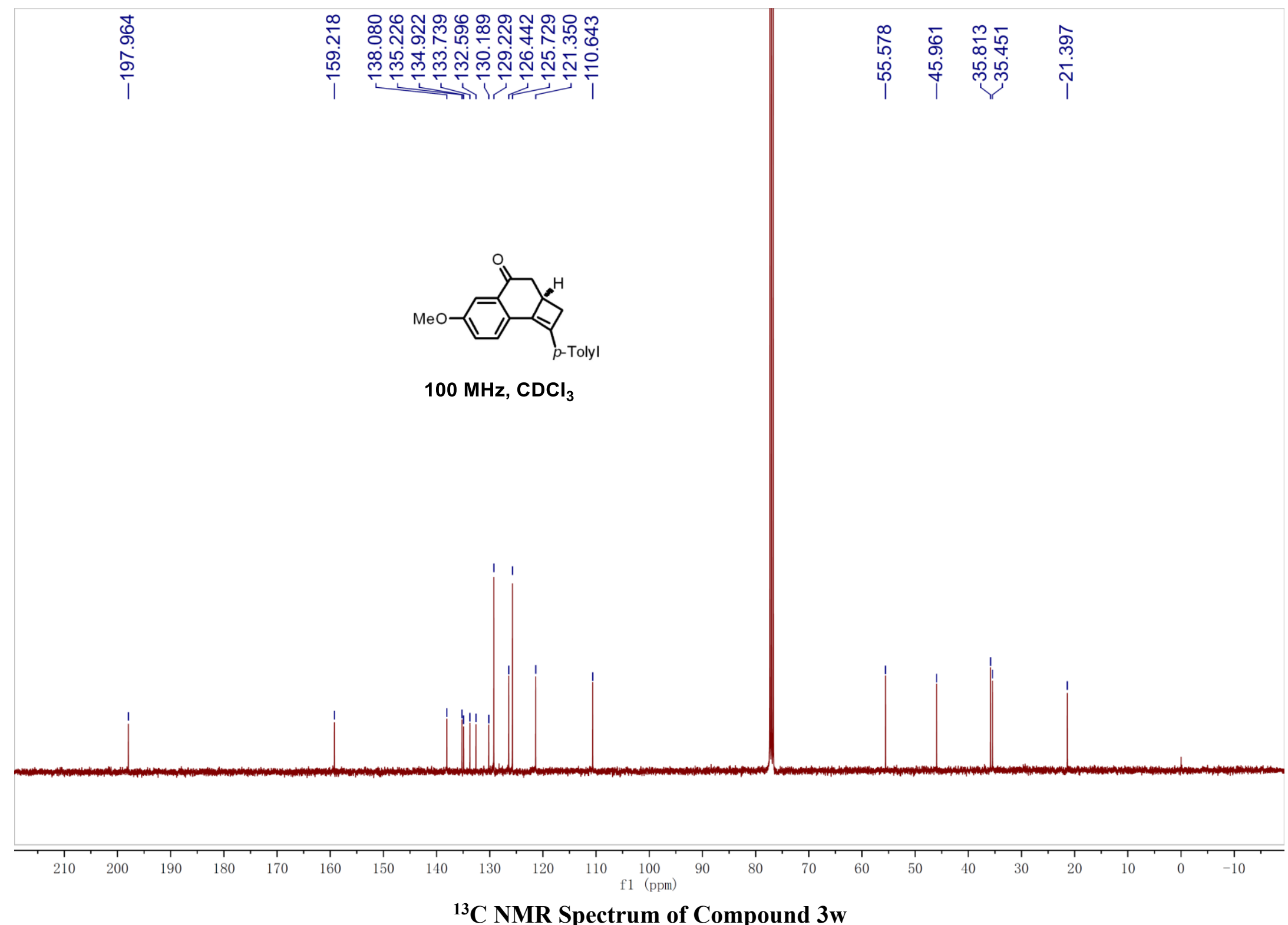


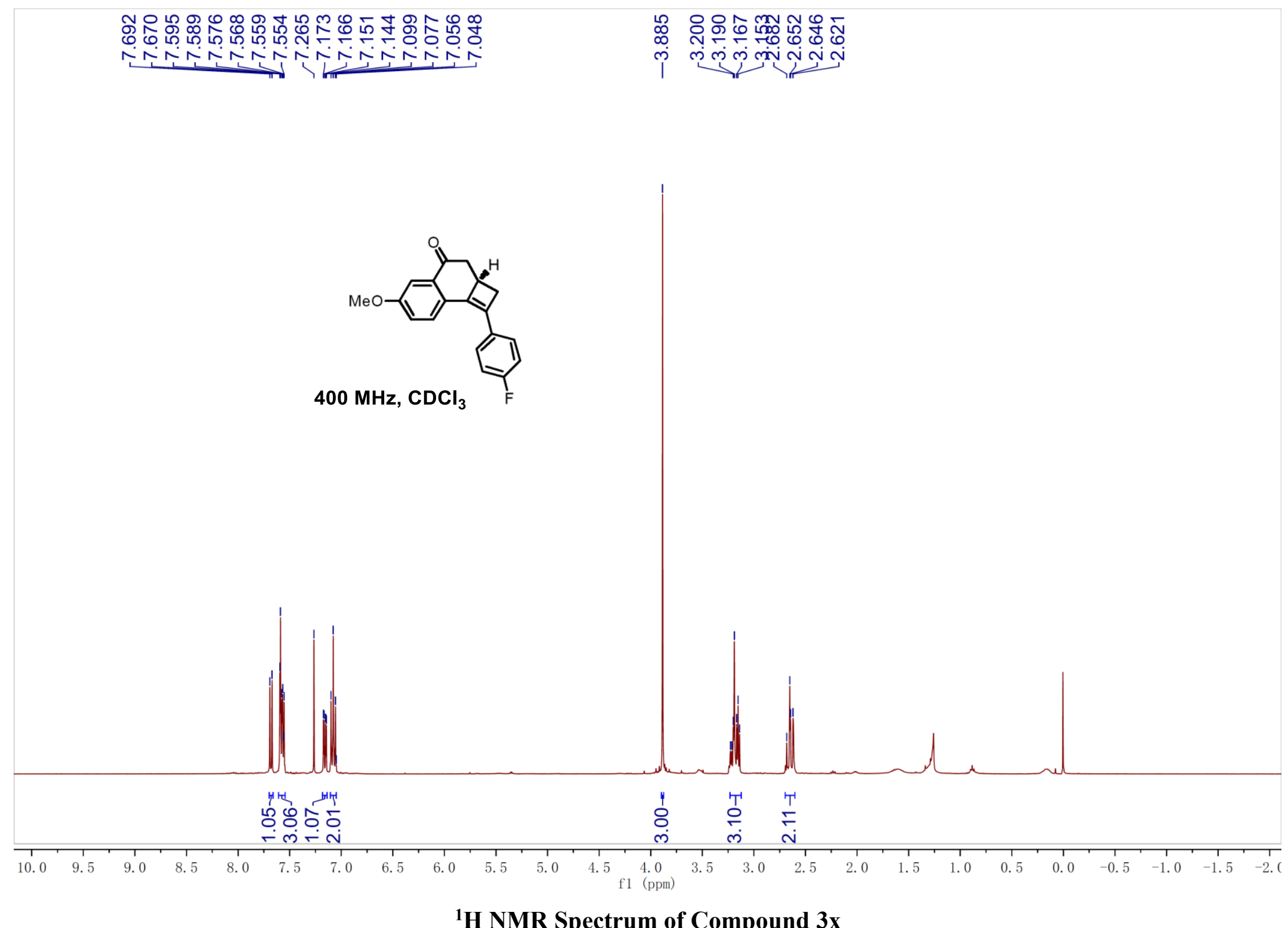

591 


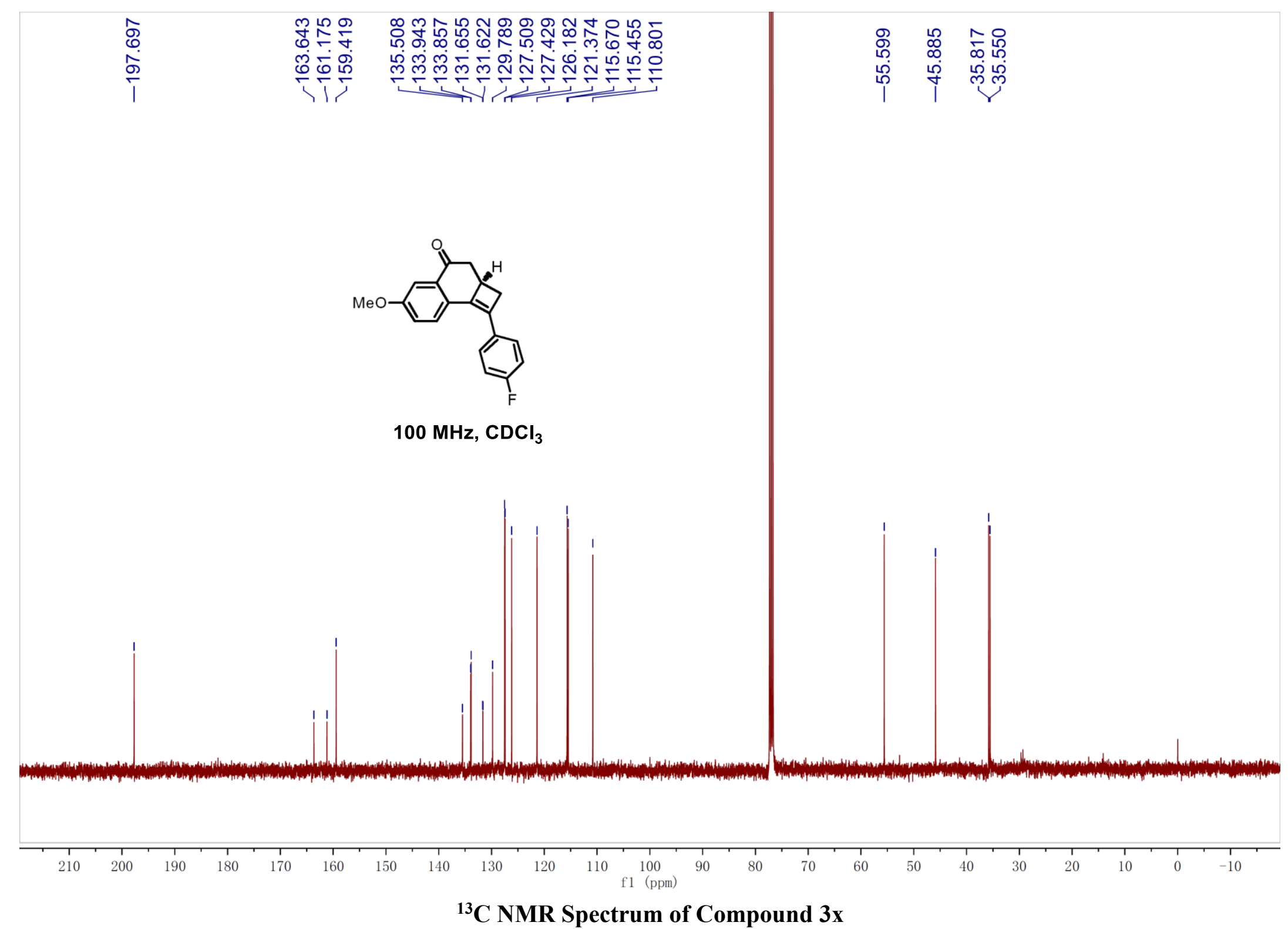




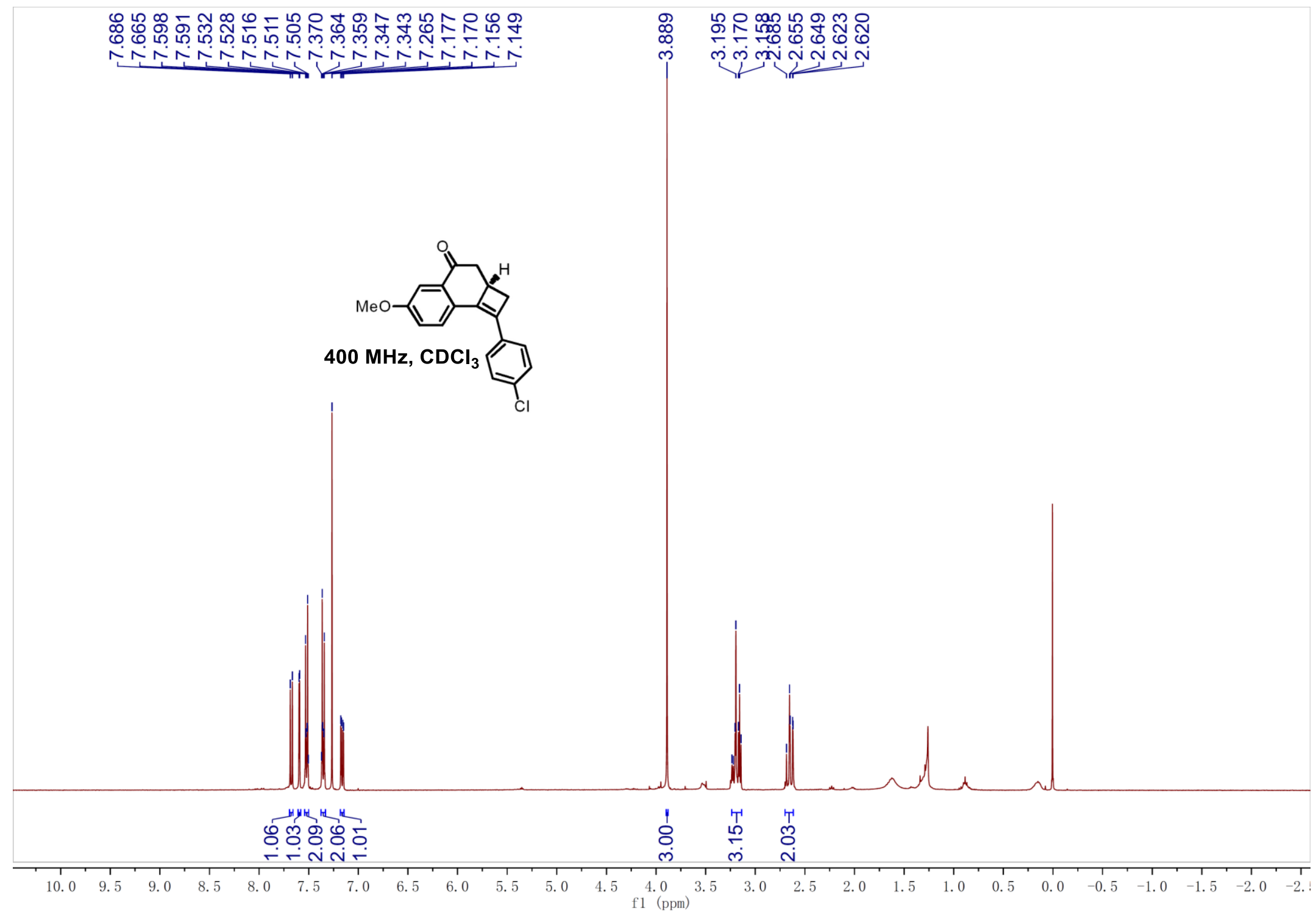

${ }^{1} \mathrm{H}$ NMR Spectrum of Compound $3 y$ 


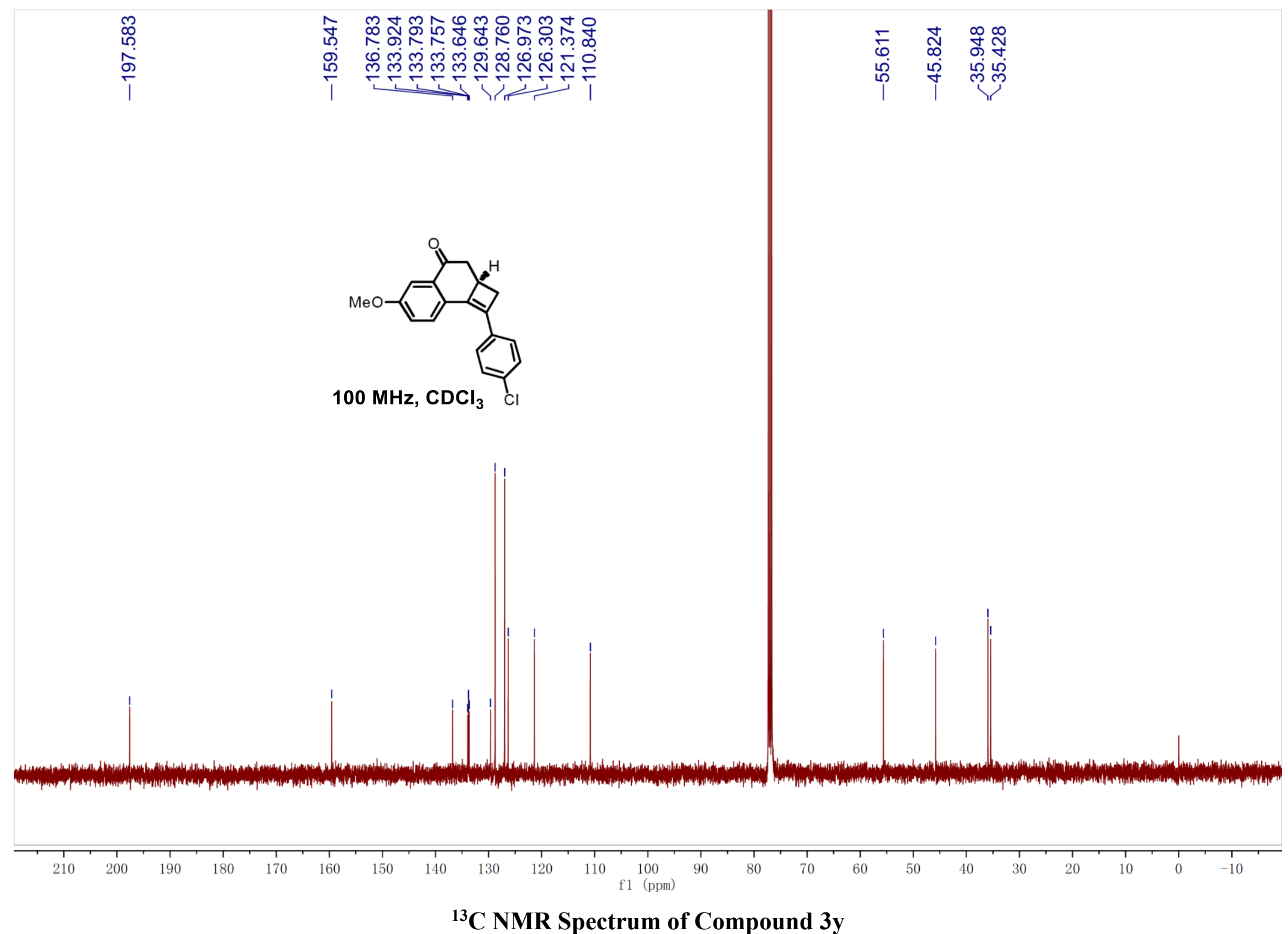




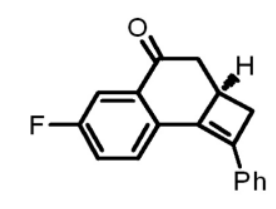

$400 \mathrm{MHz}^{\mathrm{CDCl}_{3}}$

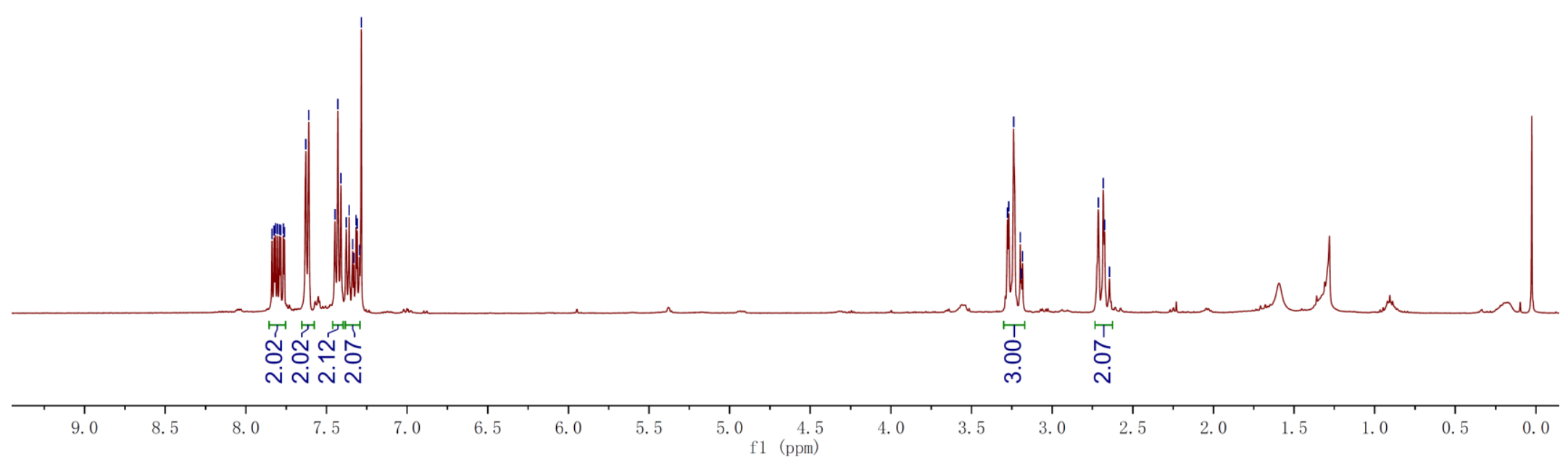

${ }^{1}$ H NMR Spectrum of Compound $3 z$ 

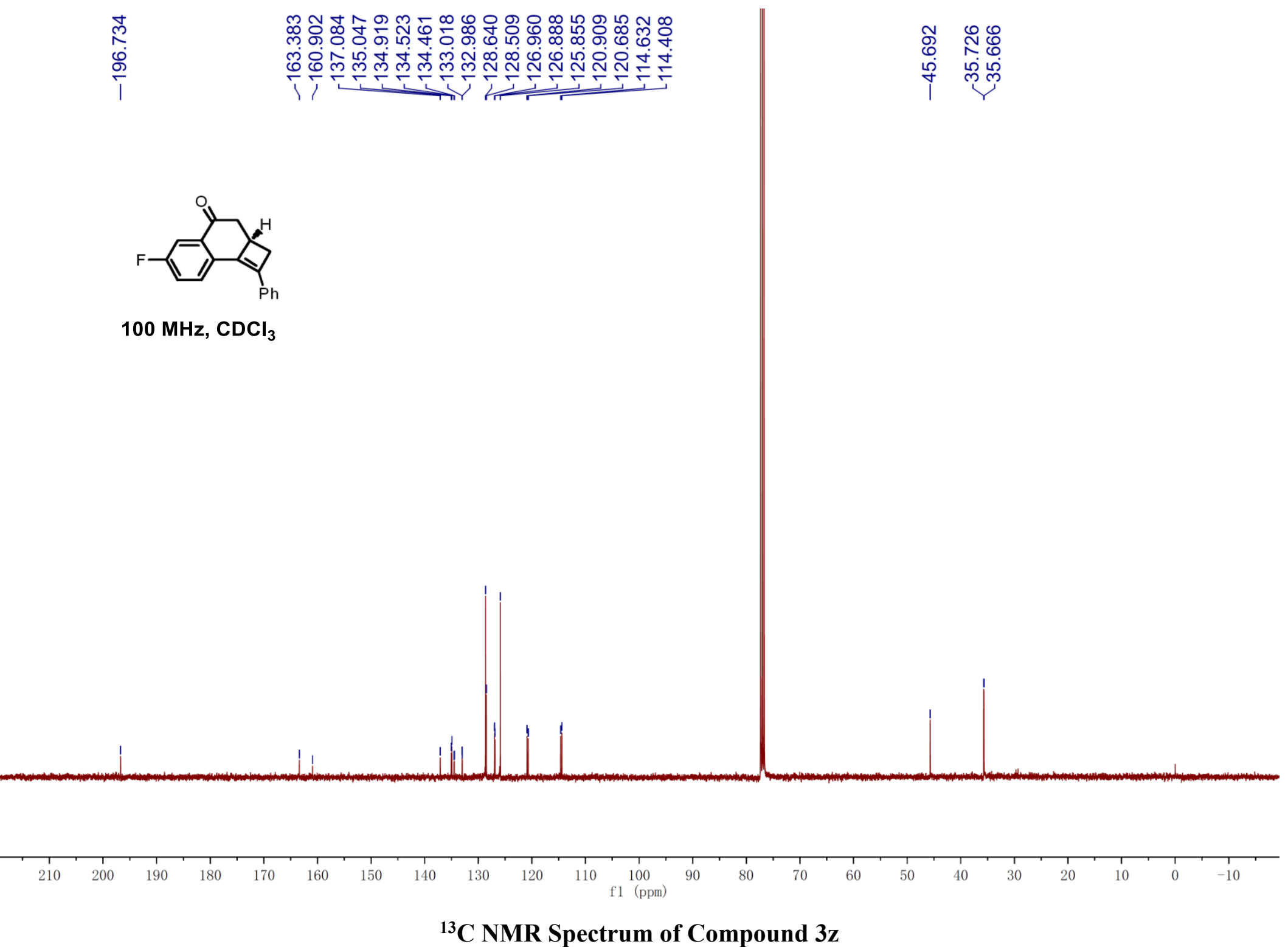


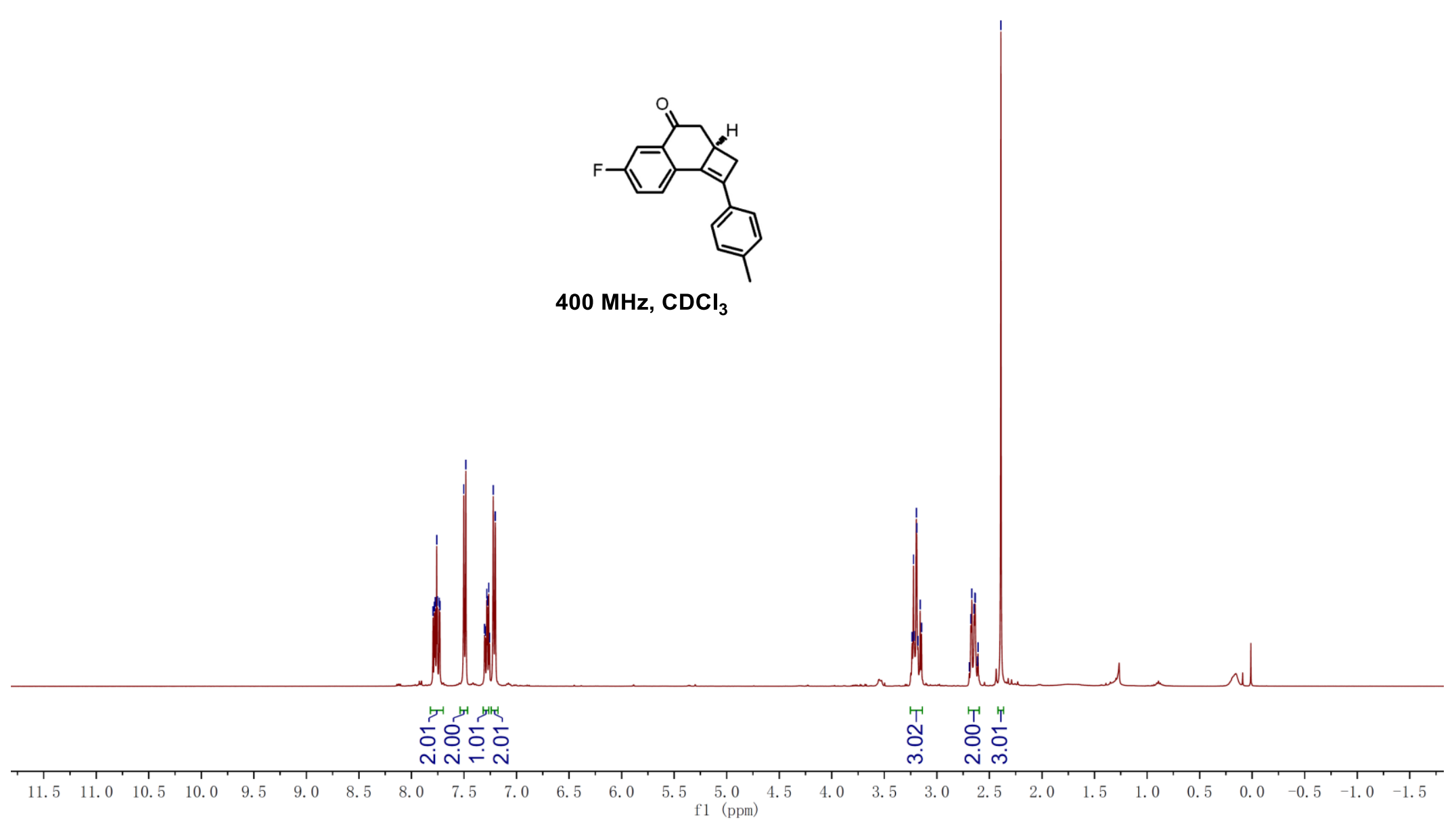

${ }^{1} \mathrm{H}$ NMR Spectrum of Compound 3aa 


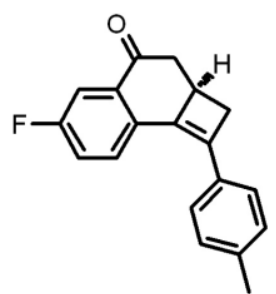

$100 \mathrm{MHz}^{\mathrm{CDCl}_{3}}$

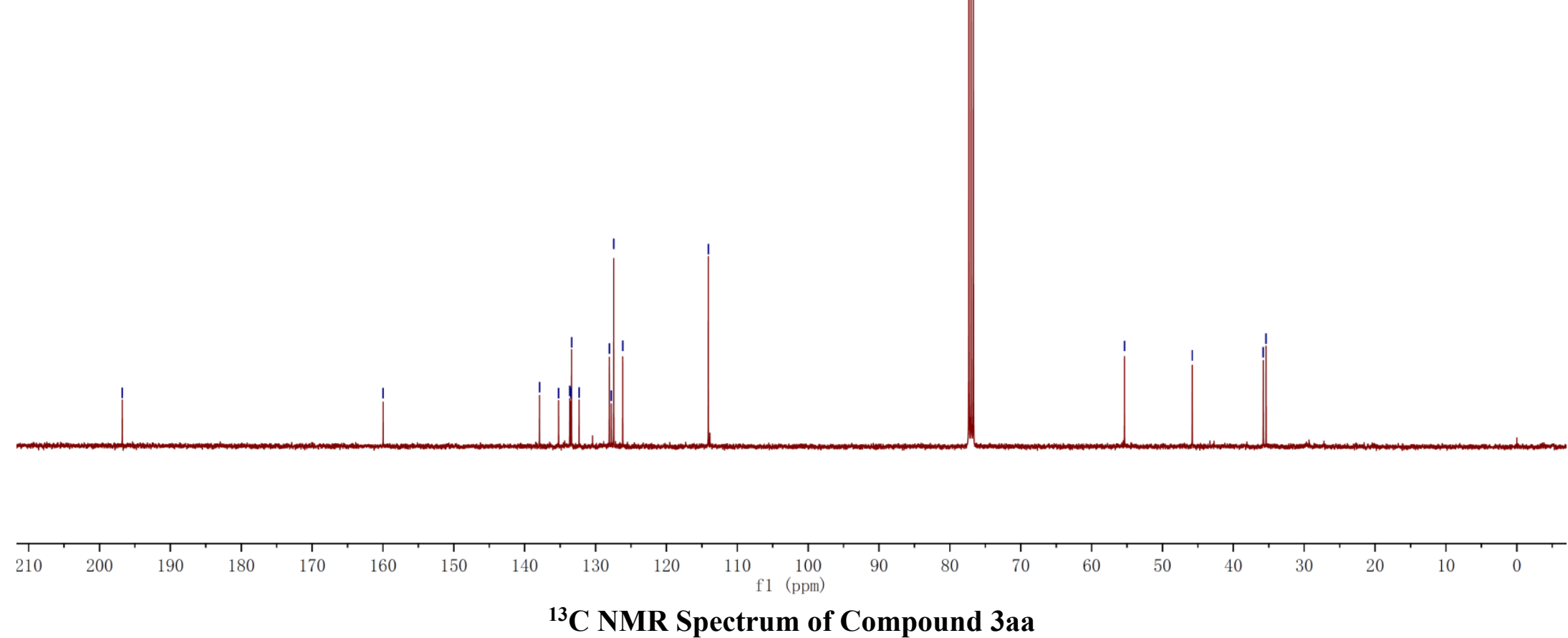




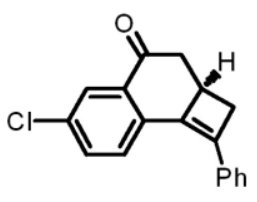

$400 \mathrm{MHz}, \mathrm{CDCl}_{3}$

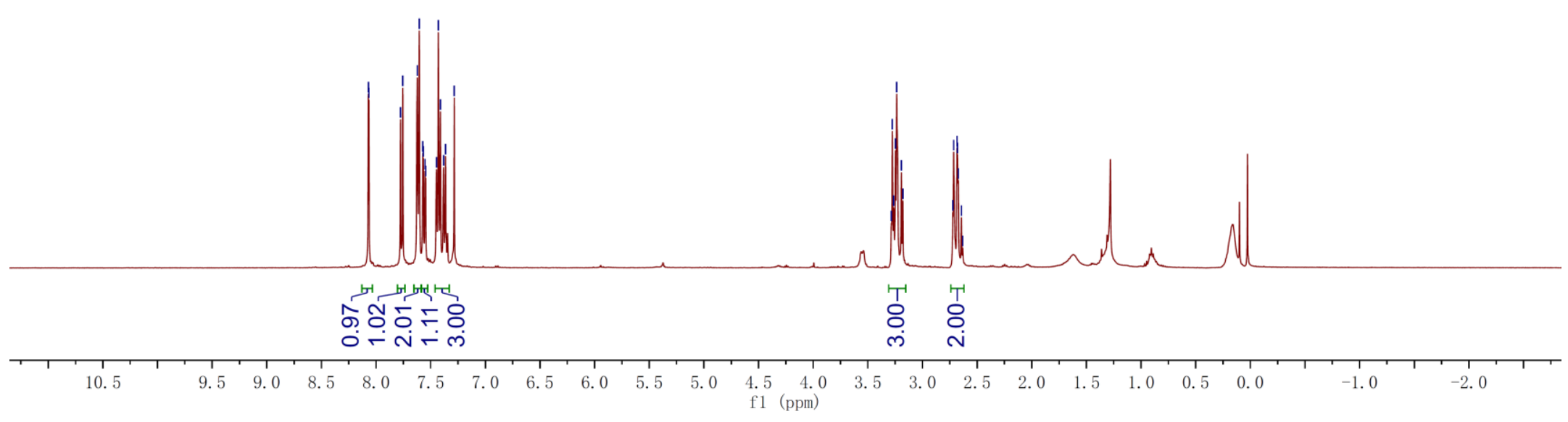

${ }^{1} \mathrm{H}$ NMR Spectrum of Compound 3bb 


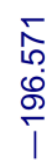

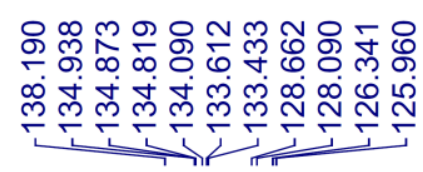

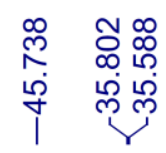

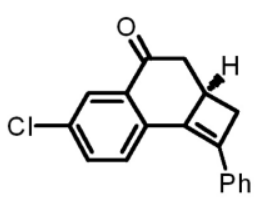

$100 \mathrm{MHz}, \mathrm{CDCl}_{3}$
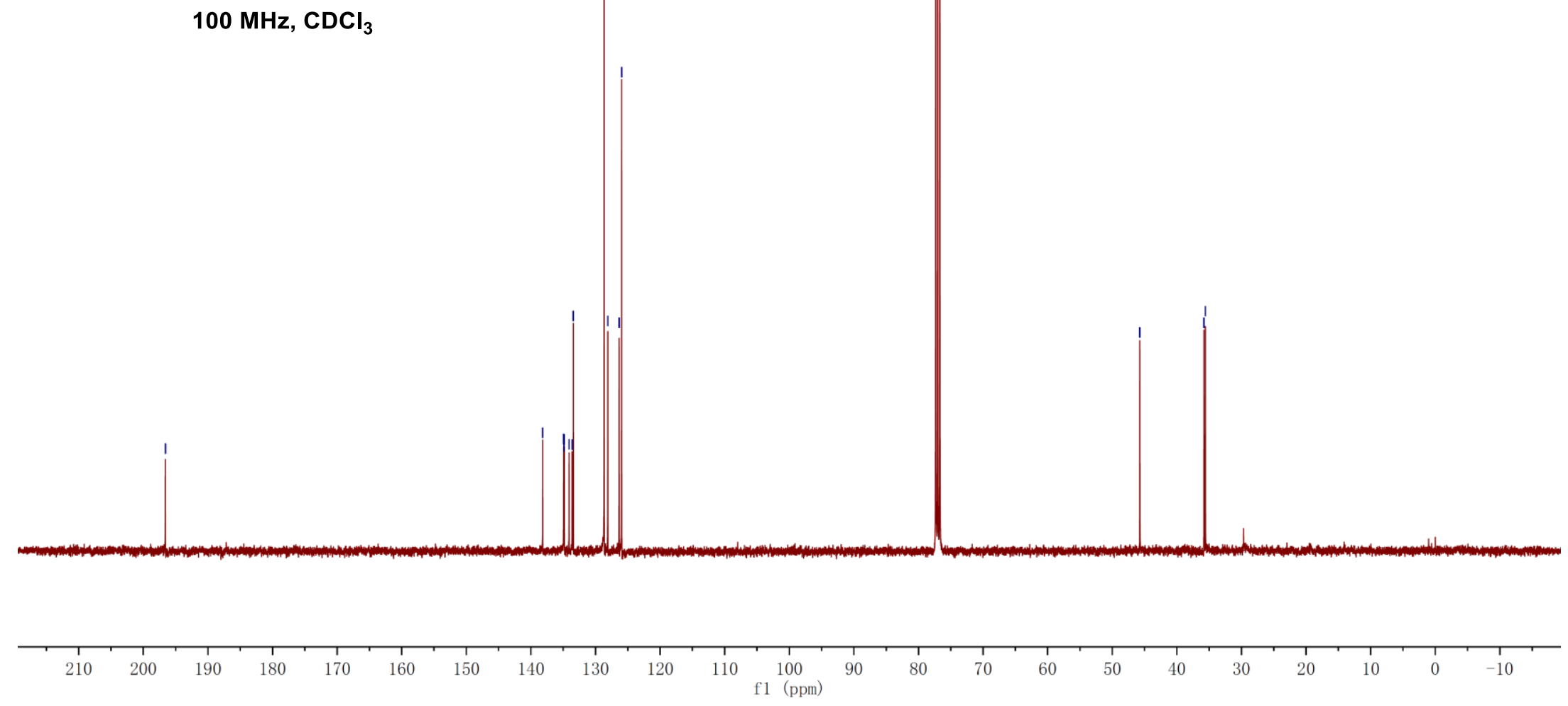

${ }^{13} \mathrm{C}$ NMR Spectrum of Compound $3 \mathrm{bb}$

S100 


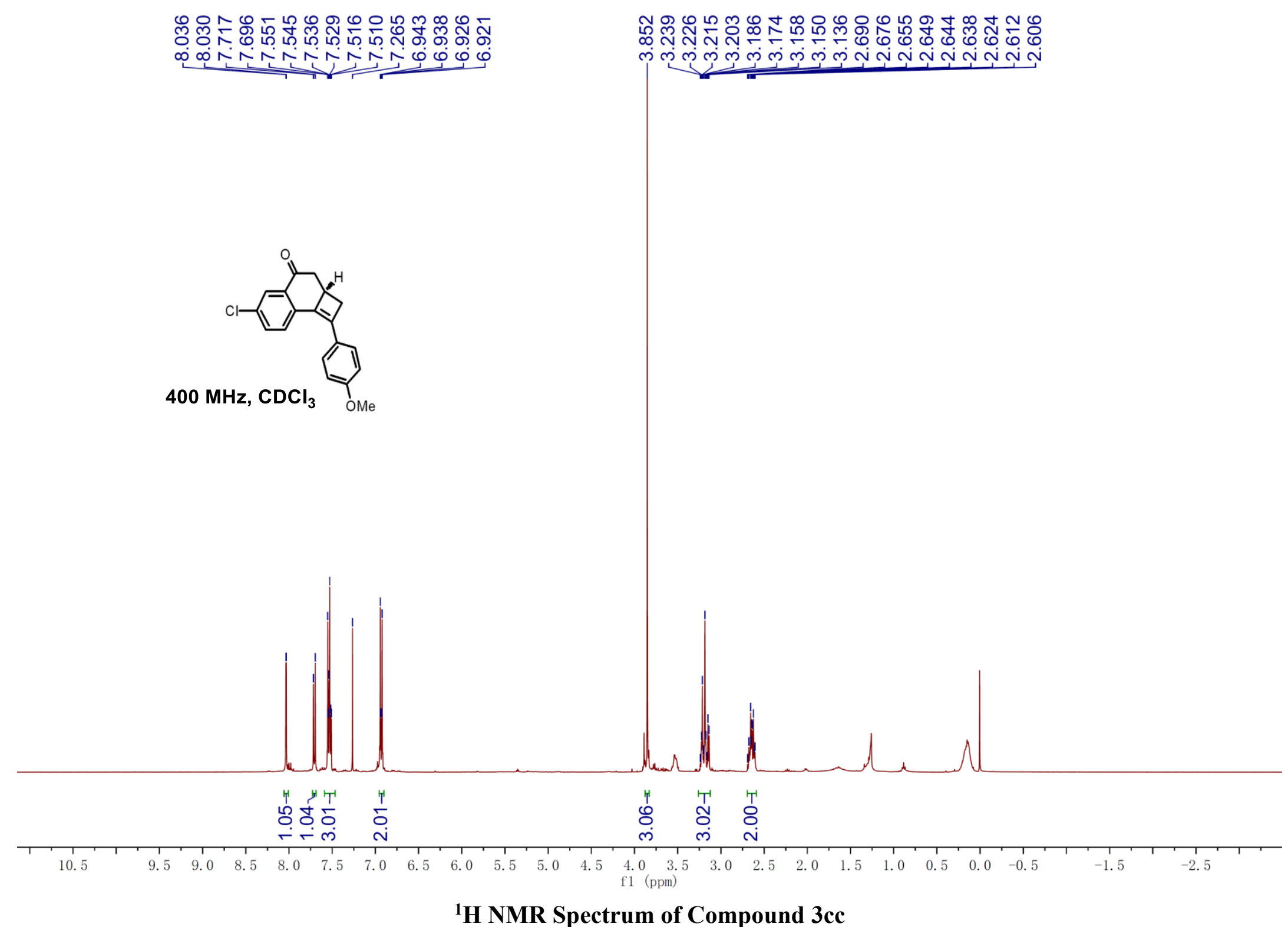




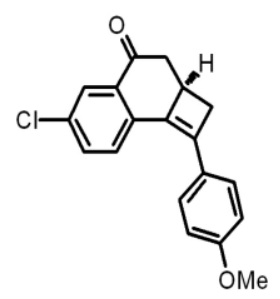

$100 \mathrm{MHz}^{\mathrm{CDCl}_{3}}$
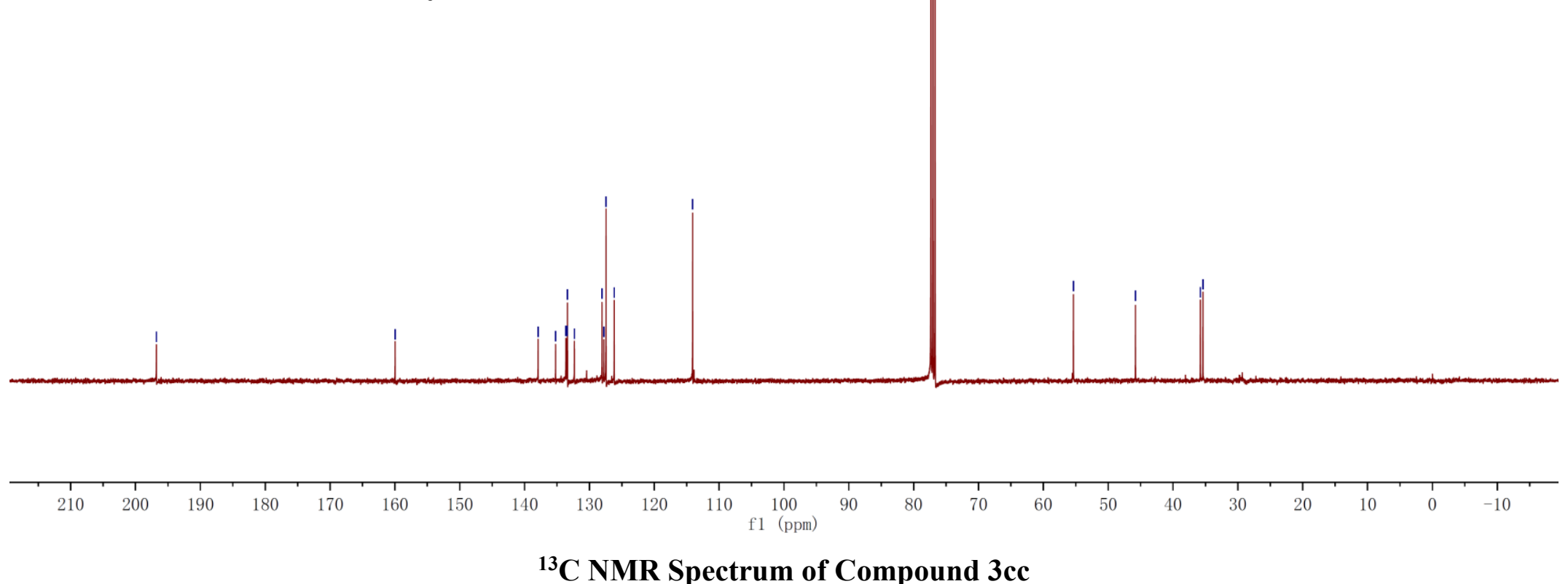


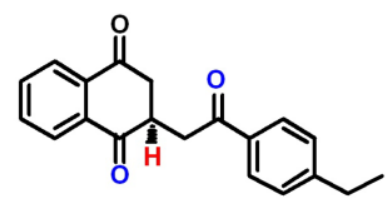

$400 \mathrm{MHz}, \mathrm{CDCl}_{3}$

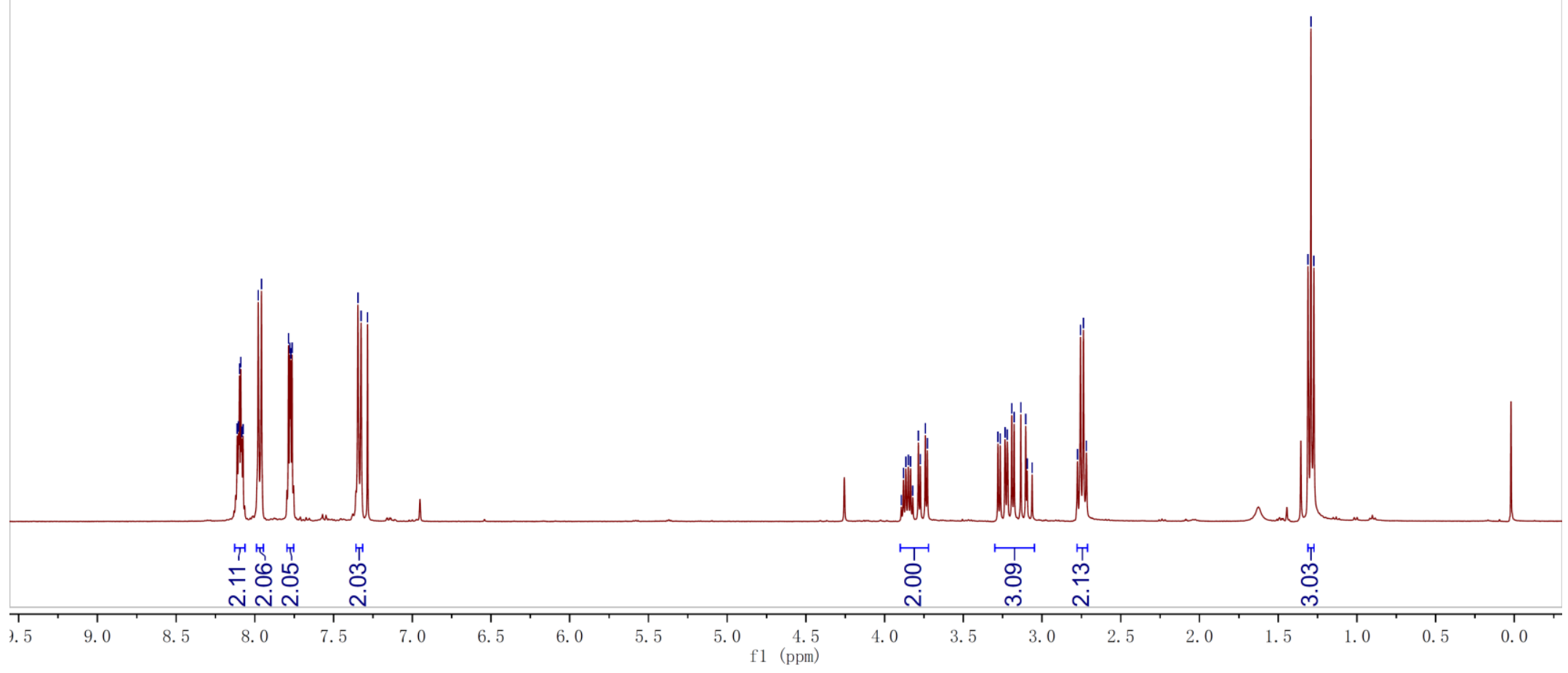

${ }^{1} \mathrm{H}$ NMR Spectrum of Compound 4 


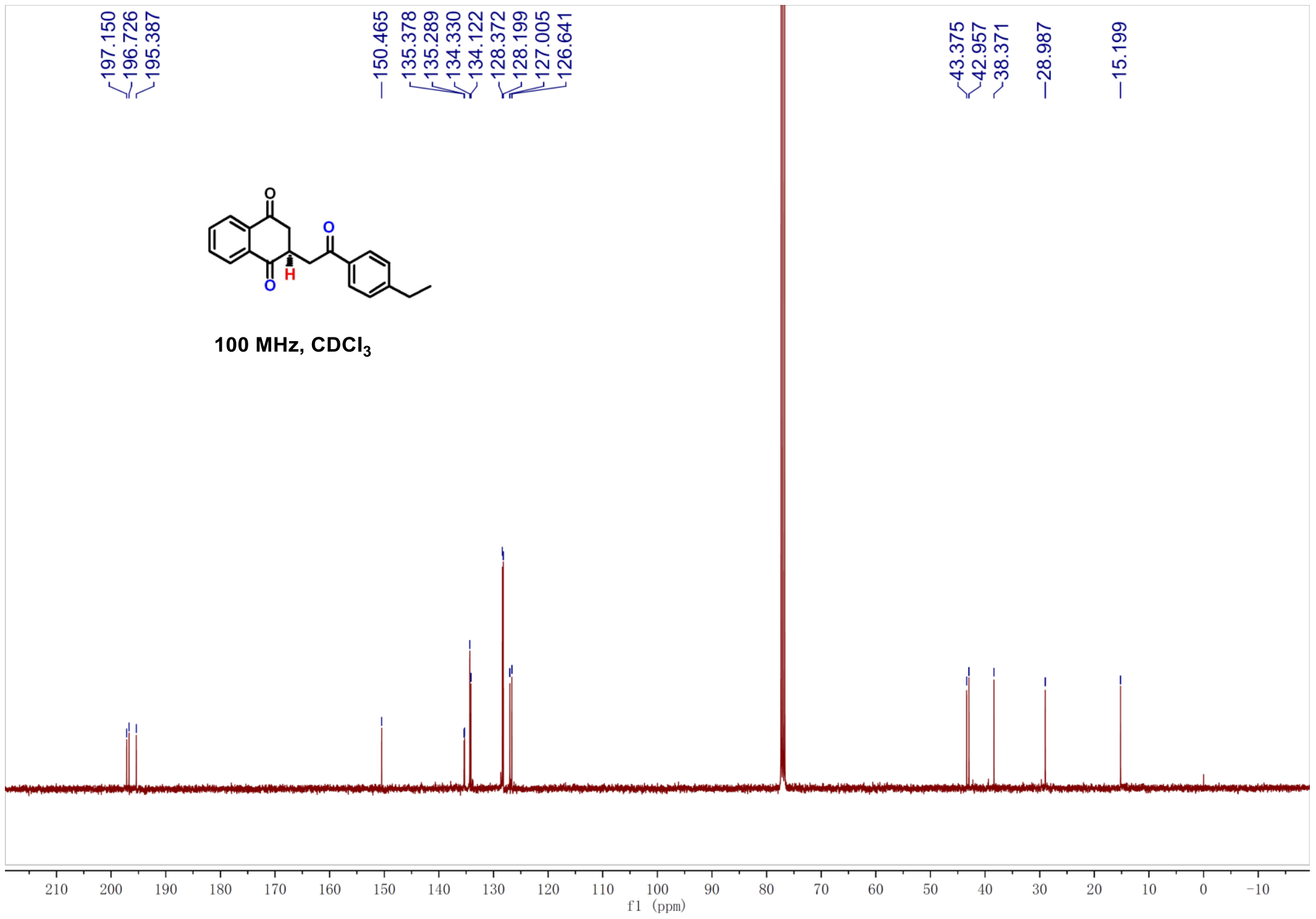

${ }^{13}$ C NMR Spectrum of Compound 4

S104 